Jürgen Weber

\title{
Begriff und Konstruktion
}

Rezeptionsanalytische Untersuchungen zu Kant und Schelling

Göttingen 1998 
Notiz

Der Text entspricht der leicht bearbeiteten Fassung meiner Dissertation, die ich 1993 dem Fachbereich Historisch-Philologische Wissenschaften der Georg-August-Universität Göttingen zur Erlangung des philosophischen Doktorgrades vorgelegt habe. J.W.

Referent: $\quad$ Prof. Dr. Konrad Cramer

Korreferent: Prof. Dr. Wolfgang Carl

Tag der mündlichen Prüfung: 21.12.1994, 13.04.1995 


\section{Inhalt}

$\begin{array}{ll}\text { Einleitung } & 5\end{array}$

1. Metaphysik als Transzendentalphilosophie 10

1.1. Kants Kritik des metaphysischen Dogmatismus 10

1.2. Das Faktum der Erfahrung 12

1.3. Schranken und Grenzen $\quad 15$

1.4. Die Grenzen metaphysischer Anfangsgründe 17

2. Was sind metaphysische Anfangsgründe der Naturwissenschaft? 20

2.1. Zielsetzungen 20

2.2. Theoriestatus 25

2.3. Verfahren 28

3. Der Begriff der metaphysischen Konstruktion 36

3.1. Textkritik 36

3.2. Interpretationen (1965-1990) 38

3.3. Plaaß' "Vermutung" 42

4. Konstruktion der Begriffe (I): "Darstellung des Begriffs in der Anschauung a priori" $\quad \mathbf{4 4}$

4.1. Zu Kants Bestimmung der mathematischen Erkenntnis als 'Konstruktion der Begriffe' 44

4.2. Zu Hintikkas Interpretation des Kantischen Konstruktionsbegriffs 46

4.3. Kants transzendentallogische Deutung des euklidischen Beweisverfahrens $\quad 51$

5. Der Begriff der Konstruktion in Kants Metaphysischen Anfangsgründen der Naturwissenschaft $\quad \mathbf{6 2}$

5.1. Die 'bloß metaphysische Erklärung' des Begriffs der Materie 62

5.2. 'Bewegung als Erscheinung' und 'Bewegliches als Gegenstand der Erfahrung' 70

5.3. Die Metaphysischen Anfangsgründe der Phänomenologie als 'physiologische Methodenlehre' 72

5.4. Die metaphysisch-dynamische Erklärungsart des Begriffs der Materie 76

5.5. Die dynamische Interpretation des Massenbegriffs 79

5.6. Das Konfliktmodell zweier ursprünglich bewegender Kräfte 84

5.7. Bewegung als "Beschreibung eines Raumes" 89 
6. Schellings Projekt einer transzendentalen Konstruktion 94

6.1. Die Frage nach den Bedingungen der Möglichkeit 94

6.2. Die absolute Konstruktion. Forschungsprobleme 99

7. Konstruktion der Begriffe (II): "Gleichsetzung des Begriffs und der Anschauung" 106

8. Die Transformation des Konstruktionsbegriffs (1796/97) 120

8.1. Ein Textvergleich: KrV: A713f. B741f. - Konstruktion: SW V 127f. 120

8.2. "Empirisch ist das, wodurch Objecte möglich sind" (1795) 124

8.3. 'konstruieren' als 'ursprünglich vorstellen': Becks Interpretation des Kantischen Konstruktionstheorems (1796) 129

8.4. 'ursprünglich vorstellen' als 'ursprünglich (transzendental) konstruieren': Schellings Interpretation der Theorie des ursprünglichen Vorstellens (1797) 135

8.5. 'ursprünglich vorstellen' als 'konstruieren': Becks zweiter Kommentar zu Kants Metaphysischen Anfangsgründen der Naturwissenschaft (1796) 138

8.6. Schellings Interpretation des Konstruktionsbegriffs in Kants Metaphysischen Anfangsgründen der Naturwissenschaft (1797)

9. Selbstkonstruktion als Prinzip der Transzendentalphilosophie 148

9.1. Konstruktion als Demonstration 148

9.2. Jacobis Kritik der demonstrativen Methode 153

$\begin{array}{ll}\text { 9.3. Selbstkonstruktion } & 156\end{array}$

$\begin{array}{ll}\text { Siglen und Kurztitel. Zitierweise und Zeichen } & 165\end{array}$

$\begin{array}{ll}\text { Literaturverzeichnis } & 167\end{array}$ 


\section{Einleitung}

Der Aufstieg der Naturwissenschaften im 17. und 18. Jahrhundert schien eng mit ihrer Emanzipation von der Metaphysik verknüpft zu sein; und je erfolgreicher die experimentelle Forschung war, desto metaphysikfeindlicher war sie eingestellt. Der Erfolg der Naturwissenschaften wurde zur Krise der Metaphysik.

Kants Antwort war frappierend: Er vertrat eine wissenschaftssystematische Doktrin, die allein die Physik Newtonscher Prägung zum Paradigma 'eigentlicher' Naturwissenschaft machte; Chemie und Psychologie wurden als unwissenschaftlich ausgegrenzt. Den Geltungsanspruch physikalischer Aussagen stellte er jedoch mit dem Argument in Frage, daß der Physiker bei der Theorienbildung notwendig, doch 'unbewußt' und daher unkritisch auf metaphysische Prinzipien rekurriere. Kant koppelte so das Legitimitätsdefizit der Metaphysik kurzerhand an das der Naturwissenschaft. Die Behebung dieser Defizite stand und fiel demnach auch mit der 'Reform' der Metaphysik als Wissenschaft, deren Grundzüge er in der $\mathrm{KrV}$ vortrug.

War auch Kants Versuch, den Kanon naturwissenschaftlicher Disziplinen zu beschneiden, historisch bald überholt, so erwies sich seine Sichtweise der Mathematik als Ingredienz jeder Form von Wissenschaft für die moderne Wissenschaftsauffassung als ebenso grundlegend wie verfänglich. Vereinfachend gesagt, konnte danach nur das, was quantifizierbar war, auch eigentlich begriffen werden. Was sich dem mathematischen Kalkül entzog, wurde jenseits der 'Grenzen der Vernunft' ins Reich der Flügelpferde verbannt. Auf seiten der Gegenaufklärer geriet dieser Verlust des Unkalkulierbaren und vermeintlich Irrationalen zur Handhabe gegen die 'Selbstinitiation der Vernunft' in der Rechtfertigung des vernunfteigenen Kriterienkatalogs wissenschaftlicher Forschung. ${ }^{1}$ Jacobis Analyse des Kantischen Konstruktionstheorems in der "Beylage VII" seiner Spinoza-Briefe ist denn auch zu einem Topos der modernen Wissenschaftskritik geworden:

"Die Construction eines Begriffes überhaupt ist das a priori aller Constructionen; und die Einsicht in seine Construction giebt uns zugleich auf das gewisseste zu erkennen, daß wir unmöglich begreifen können, was wir zu construiren nicht im Stande sind. Darum haben wir von Qualitäten, als solchen, keine Begriffe; sondern nur Anschauungen. [...] Wenn wir sagen, daß wir eine Qualität erforscht haben, so sagen wir damit nichts anders, als wir haben sie auf Figur, Zahl, Lage und Bewegung zurückgeführt, und darin aufgelöst; also: wir haben die Qualität objektiv vernichtet." 2

Schelling teilte Jacobis Bedenken, die für ihn jedoch Anlaß nicht zur Abkehr, sondern zur Radikalisierung des Kantischen Ansatzes waren. Konstruktion als genuin mathematische Methode sollte nun nicht mehr nur der philosophischen Interpretation fähig sein, Konstruktion wird zum universellen Methodenbegriff und zum Interpretament der wissenschaftlichen Philosophie selbst.

1 Zu den 'Aporien der Aufklärung' aus der Sicht Herders und Hamanns vgl. Gaier (1989) 261-276; zur ideologiegeschichtlichen Debatte über Aufklärung, Gegenaufklärung, Dialektik der Aufklärung vgl. Schmidt (1989); zur Kontinuität von Aufklärung und Idealismus vgl. Jamme/Kurz (1988).

2 Jacobi (1789) 420. 
Kants und Schellings Deutungen des geometrischen Konstruktionsbegriffs sind das Thema dieser Untersuchungen. Als roter Faden zieht sich die Erkundung des Problemfeldes durch, in dem sich Schellings Rezeption, Kritik und Weiterbildung des Kantischen Konstruktionstheorems vollziehen. Beide Konzeptionen sowie Umfang und Vorgehen der Rezeptionsanalyse sollen hier kurz vorgestellt werden.

'Konstruktion der Begriffe' ist die Formel, mit der Kant die mathematische Erkenntnisart in ihrer spezifischen Differenz zur philosophischen Erkenntnisart kennzeichnet:

"Die philosophische Erkenntnis ist die Vernunfterkenntnis aus Begriffen, die mathematische aus der Konstruktion der Begriffe. Einen Begriff aber konstruieren heißt: die ihm korrespondierende Anschauung a priori darstellen." (A713 B741)

Es ist zu fragen, worin das Kriterium der Differenz beider Erkenntnisarten besteht und wie die Beziehung der Erkenntnisarten präzisiert werden muß, damit deren Differenz gerade durch das Verfahren der 'Darstellung in der Anschauung a priori' auch explizierbar ist. Bei seiner Deutung des geometrischen Konstruktionsbegriffs orientiert sich Kant an der Funktion, die Konstruktion als Teilschritt des euklidischen Beweisverfahrens erfüllt. Kants Formulierung des Konstruktionstheorems als 'Darstellung des Begriffs in der Anschauung a priori' ist Ausdruck und Resultat dieser Deutung in der Perspektive der transzendentalen Theorie der Erfahrung. Dadurch kann er die Differenz von philosophischer und mathematischer Erkenntnisart innerhalb und mit den Mitteln der transzendentalen Logik beschreiben und begründen. Als "Probe" (B110) und Realisierung "eigentlich der Transzendentalphilosophie"3 will Kant denn auch die $M A N W$ verstanden wissen. Darin versucht er am Modell der Newtonschen Mechanik zu zeigen, wie es sich erklären läßt, daß wir ein ideelles System mathematischer Größen und Gesetze auf das, was wir als Natur erfahren, erfolgreich anwenden können. Die Aufklärung dieses 'Faktums' der Geometrisierung der Natur verbindet er mit der tiefergehenden Nachfrage nach dem Sachverhalt, den eine physikalische Theorie, die vorgeblich bloß auf mathematischen Prinzipien beruhe, zwar stets voraussetzt, aber zu rechtfertigen gar nicht imstande ist: Wie läßt sich verstehen, daß wir naturwissenschaftliche Hypothesen überprüfen und stützen oder widerlegen können, also in der Lage sind, objektiv gültige Verifikationskriterien für physikalische Begriffe anzugeben, deren Bedeutungsgehalt wir in einer formalen Axiomatik definieren und doch auf der Ebene empirischer Experimente korrigieren können?

Kant entwickelt ein kritisches Konzept von Empirizität, das die Kriterien empirischer Wahrheit dessen angibt, was wir als 'Faktum der Erfahrung' betrachten. Faktum ist es nicht im vorwissenschaftlichen Sinne, sondern nur sofern es den Bedingungen mathematischphysikalischer Beschreibung und damit den Bedingungen mathematischer Konstruktion in der Anwendung auf den Objektbereich der Naturwissenschaft genügt. Schelling folgt insoweit der Kantischen Deutung des Konstruktionsbegriffs, als er dessen Funktion und die Evidenz mathematischer Erkenntnis aus der beweistheoretischen Konzeption des euklidischen Modells herleitet. Allerdings begreift er die 'Darstellung in der Anschauung' 
nicht mehr nur als ein Moment des Bedingungsgefüges von Konstituenten und Regulativen in Urteilen über empirische Sachverhalte, das, wie vielfach unterstellt, im Sinne einer 'Logogenese' als Analyse der Bedingungen der Möglichkeit solcher Sachverhalte aufzuklären wäre. ${ }^{4}$ Konstruktion ist keine Beschreibung irgendeiner Genese. Es geht um den Aufweis eines Wahrheitskriteriums in unserem Wissen um die Sachverhalte. Konstruktion ist Demonstration: "sie erklärt nicht [...], sie beweist, daß es so sey."

Die Karriere des mos geometricus ist durch Kants vermeintlich radikale Trennung mathematischer und philosophischer Verfahrensweisen, anders als vermutet ${ }^{6}$, nicht beendet worden; im Gegenteil, Kants Akzentuierung des konstruktiven Moments hat die Universalisierung der mathematischen Methodik bestätigt. Bereits 1798 formuliert Schelling programmatisch: Man werde "bald kein anderes als mathematisches Wissen kennen, und der transcendentale Geist, der in der Mathematik zuerst seinen Ursprung nahm, wird so zu seiner Quelle zurückkehren. ${ }^{77}$ Dieser Überschlag in der Sache prägt auch den hermeneutischen Zugriff auf die Kantischen Texte. Schellings Kant-Lektüre geht von der Annahme aus, "daß Kant, wenn er ein vollendetes Bewußtseyn seiner Philosophie hätte und der Reflexion darüber fähig wäre, construiren müßte". ${ }^{8}$ Schelling wird Kants Texte umgekehrt da, wo er sich mit Kant einig glaubt, so lesen, als ob Kant (im Sinne Schellings) konstruiere.

Näheren Aufschluß über diese Rezeptionshaltung gibt eine vergleichende Analyse von Schellings und Höijers Kritik des Kantischen Konstruktionstheorems. Textgrundlage sind Höijers Abhandlung über die philosophische Construction (1801) und Schellings Rezension dieser Abhandlung, die unter dem Titel Über die Construction in der Philosophie (1802) im gemeinsam mit Hegel herausgegebenen Kritischen Journal der Philosophie erschienen ist. Das vergleichsweise gut überschaubare Setting der Rezeption (Schellings durch Höijer vermittelte Kant-Kritik) erlaubt es, Schellings Redeweise bis in einzelne sprachliche Wendungen hinein nachvollziehbar $\mathrm{zu}$ machen und den philosophischen Konstruktionsbegriff schärfer, als dies bislang möglich war, zu konturieren. Diese philologisch orientierten Untersuchungen werden verbunden mit einer Analyse des Problembestandes, dessen Bewältigung es Schelling dringlich erscheinen ließ, Konstruktion aus dem mathematischen Kontext herauszulösen und als philosophischen Methodenbegriff zu etablieren.

Dieser Vorgang ist deshalb so aufsehenerregend, weil Schelling (übrigens gegen die Stellungnahmen Fichtes und Hegels) das eigene Methodenkonzept gerade im Rückgriff auf Kants Konstruktionstheorem entwickelt, das doch die Differenz von philosophischer und mathematischer Erkenntnis endgültig festgeschrieben haben sollte: Wie läßt sich also die Transformation des Konstruktionsbegriffs durch Schelling als Konsequenz gerade der Kantischen Unterscheidung beider Erkenntnisarten verstehen?

4 Den Begriff der 'Logogenese' führt Krings (1982) ein. Ausführlichere Studien zu Schellings Konstruktionsbegriff: Salvucci (1958), Ende (1973), Taureck (1975), Verra (1979).

5 System 1802: SW IV 345; vgl. 407: "Der Demonstration geht die Construction nicht voran, sondern beides ist eins und unzertrennlich."

Arndt (1971) 160, Engfer (1982) 67.

Übersicht: SW I 463f. = AW IV 181.

Konstruktion: SW V 140. 
Schelling stößt auf den Konstruktionsbegriff in seiner Auseinandersetzung mit Kants $M A N W$. Man muß geradezu von einer Entdeckung des Konstruktionsbegriffs sprechen, dessen Herauslösung aus dem mathematischen Kontext sich auf der Folie eines eigentümlichen Syndroms offener Problemstellungen der Kantischen Philosophie vollzieht: So schweigt sich Kant in der $K r V$ über die "eigentümliche Methode der Transzendentalphilosophie" (A738 B766) aus; die $K r V$ liefere nur die 'Propädeutik' zum System der Philosophie, nicht das System selbst. ${ }^{9}$ In der Perspektive der frühidealistischen Kant-Interpretation erscheint die Propädeutik aber als das 'Resultat' des Systems, dessen "Prämissen fehlen". ${ }^{10}$ Die $M A N W$ sollen Kant zufolge die formellen Vorgaben der $\mathrm{KrV}$ umsetzen, indem sie zeigen, wie den Lehrsätzen und Begriffen "eigentlich der Transzendentalphilosophie" Sinn und Bedeutung gegeben wird. ${ }^{11}$ Dementsprechend gelten aus idealistischer Sicht die $M A N W$ als materieller oder realphilosophischer Teil der Transzendentalphilosophie. Die Analyse ihrer Resultate sollte nun die Folie abgeben, auf der die unentschiedene Prämissenfrage zu lösen war. Kants Begründungsversuch der mathematischen Konstruktion des dynamischen Begriffs der Materie konnte so als 'Modifikation' und 'Beispiel' philosophischer Konstruktion aufgefaßt werden. ${ }^{12}$

In der frühidealistischen Debatte über die Grundlagen der Kantischen Philosophie bin ich auf zwei Beiträge gestoßen, die im Rekurs auf das beschriebene Syndrom die Transformation des Konstruktionsbegriffs unmittelbar vorbereiten oder bereits vollzogen haben: Becks sog. Theorie des ursprünglichen Vorstellens (1796), in deren Kritik Schelling den Konstruktionsbegriff einführt, und Höijers Abhandlung (1801), in deren Rezension Schelling sich erstmals mit Kants Konstruktionstheorem anhand der einschlägigen Textstücke der "Transzendentalen Methodenlehre" der $\mathrm{KrV}$ auseinandersetzt.

Im dritten Band seines Erläuternden Auszug der kritischen Schriften Kants führt Beck das "Postulat des ursprünglichen Vorstellens" ein, in dem er zugleich das Fundament der Transzendentalphilosophie Kants sehen will. Nun ist Beck davon überzeugt, daß sich das "Transcendentale unsrer Erkenntniß" wie das "Princip des ursprünglichen Vorstellens" gerade am Beispiel der $M A N W$ verdeutlichen lassen. ${ }^{13}$ Im Hinblick auf Kants Materiemodell zweier konfligierender Kräfte führt Beck dann aus: "Das ursprüngliche Vorstellen aber, worauf sich der Begriff des Conflictus beyder Kräfte, wodurch ein bestimmter Raum erfüllt ist, stützt, ist eine Construction." ${ }^{14}$ Becks Interpretation des Konstruktionsbegriffs in den $M A N W$ gibt terminologisch wie systematisch den entscheidenden Impuls zur Transformation des Konstruktionsbegriffs, die Schelling im Zuge seiner Kritik der Theorie des ursprünglichen Vorstellens mit der "Foderung einer ursprünglichen (transscendentalen) Construction" auf den Begriff bringt. ${ }^{15}$ Höijers

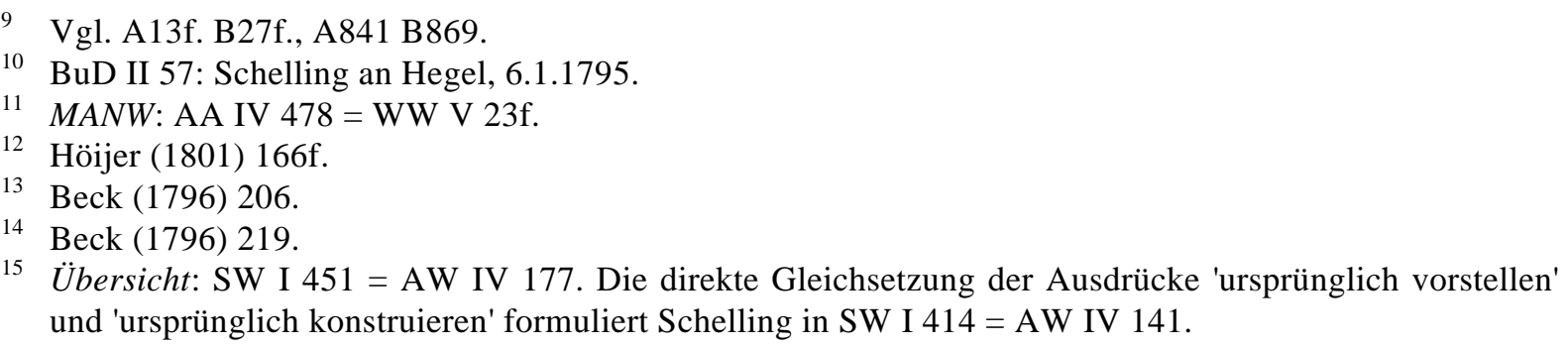


Abhandlung führt dann die heuristische Perspektive der frühidealistischen Interpretation der $M A N W$ vor Augen. In der "Einleitung" heißt es:

\begin{abstract}
"Übrigens haben wir von Kant selbst die nächste Veranlassung zu der Idee genommen, die wir hier auszuführen suchen. Er construirt in der That die Materie in seinen metaphysischen Anfangsgründen der Naturwissenschaft. Diese Construction ist aber nicht mathematisch; sie geschieht nicht im Raume und in der Zeit, denn durch dieselbe soll jener und mittelbar auch diese erfüllt werden: sie muß also philosophisch seyn." ${ }^{16}$
\end{abstract}

Es ist der mit dem Übergang der Rede von der 'Konstruktion des Begriffs der Materie' (Kant) zur 'Konstruktion der Materie' (Höijer) indizierte Sachverhalt, der die Transformation des Konstruktionsbegriffs sichtbar macht, aber keineswegs erklärt. Das Problemfeld, in dem Kants dynamische Konzeption von Materie erst zum Vehikel der Transformation werden konnte, $\mathrm{zu}$ entwickeln und $\mathrm{zu}$ beschreiben, ist der leitende Gesichtspunkt dieser Untersuchungen.

Im Schelling-Teil (Kap.6-9) beschränke ich mich auf die Detailanalyse der von Höijer vermittelten affirmativen Kant-Kritik Schellings. Diese Beschränkung ermöglicht es, die Dimension der Fragestellungen, die sich aus der Sicht der neueren Kant-Forschung an den Konstruktionsbegriff knüpfen, mit seiner frühidealistischen Lesart und der Interpretation durch Schelling zu konfrontieren. Daraus lassen sich Rückschlüsse auf das Konstruktionsproblem in den MANW ziehen, das, so meine These, nicht aus der Sicht Kants, sondern erst aus der Sicht seiner Rezipienten manifest wird. Der Rekurs auf den Problemtitel der 'metaphysischen Konstruktion', der sich im Anschluß an Plaaß (1965) in der neueren Forschung als genuiner Methodenbegriff der $M A N W$ durchgesetzt hat, drängt sich hier geradezu auf.

Im Kant-Teil (Kap.1-5) versuche ich, die Motive und Problemfelder zu erkunden, die es gerechtfertigt erscheinen ließen, Kant die Bildung und Anwendung des Zwitterbegriffs der metaphysischen Konstruktion zu unterstellen, der nach den Vorgaben seiner eigenen Theorie als contradictio in adiecto aufgefaßt werden muß. Der Vergleich beider Rezeptionslinien, die sich ungeachtet ihrer unterschiedlichen Herkunft in den Resultaten ihrer Kant-Exegese so fremd gar nicht sind, läßt folgende Schlußfolgerung zu: Die Bestimmung der mathematischen Erkenntnisart als 'Konstruktion der Begriffe' ist an die Aufklärung der Frage zu knüpfen, worin eigentlich das Kriterium der Differenz von philosophischer und mathematischer Erkenntnisart besteht und ob und inwiefern es sich innerhalb der Theorie, die es formuliert, begründen läßt. Der Sinn und die sprachliche Artikulation dieser Differenz lassen sich einsichtig machen, wenn die Erkenntnisarten nicht isoliert voneinander, sondern aufeinander bezogen expliziert werden. Die Differenz ist der transzendentalen Theorie der Erfahrung immanent. Terminologisch wie der Sache nach läßt sich Kants Redeweise der 'Konstruktion der Begriffe' als transzendentallogische Interpretation des euklidischen Beweismodells verstehen. Kant wie Schelling geht es darum, aufzuweisen, wie dieses Modell in seiner Anwendung auf das, was wir als Natur erfahren, reformuliert werden kann.

16 Höijer (1801) 6. 


\section{Metaphysik als Transzendentalphilosophie}

\subsection{Kants Kritik des metaphysischen Dogmatismus}

Mendelssohns prominentes, aber irreführendes Urteil über den "alles zermalmenden" Kant ${ }^{1}$ in Sachen Metaphysik weist auf die tiefe Verunsicherung hin, die Kants radikale Kritik der überkommenen metaphysischen Systeme unter den Spätaufklärern ausgelöst hatte. Die Konturen solcher Systeme - so lautete Kants Fazit aus der historischen Perspektive seiner Vernunftkritik - ließen "zwar Gebäude, aber nur in Ruinen" (A852 B880) erkennen.

Die abwehrende Haltung Mendelssohns und vieler seiner Zeitgenossen ist vor dem Hintergrund der für den aufklärerischen Optimismus ganz ungeheuerlichen Kompetenzbeschneidung menschlicher Rationalität freilich eher Ausdruck eigener Verstörung und so Perhorreszierung als zutreffende Analyse des Kantischen Unterfangens. ${ }^{2}$ Mochte sich Kant der skeptizistischen Entscheidung gegen alles, was Metaphysik hieß, nicht anschließen, so war doch auch unverkennbar, daß der wichtigste Repräsentant metaphysischer Denkschulen, der Dogmatismus, den eigenen Anspruch vernünftigen Argumentierens nicht einlösen konnte. In seiner rationalistischen wie empiristischen Variante hantierte der Dogmatismus mit Lehrsätzen, über deren Wahrheit und Falschheit zu entscheiden die Kriterien fehlten. Die Malaise der den zeitgenössischen Philosophiebetrieb dominierenden Doktrin war schlicht ihre Unwissenschaftlichkeit. Was die Verfechter dieser Schule vor der Öffentlichkeit so kompromittierte, konnte auch für den akademischen Lehrbetrieb nicht belanglos sein. Polemisch sprach Kant gar von Verführung der Jugend und von schleichender Vergiftung; aus seiner Sicht war es allerdings der Dogmatiker, der seinem Kritiker den Schierlingsbecher reichte. (A754 B782)

Doch hatte Kant Gründe genug, es nicht bei der bloßen Diskreditierung des Dogmatismus zu belassen. Der Befund seiner Vernunftkritik war zu prekär: Zum einen glaubte er die Fehlerquelle der dogmatischen Irrtümer in einer der Vernunft immanenten Dialektik entdeckt zu haben. Danach werde die Vernunft in den Grundfragen der traditionellen Metaphysik (Gott, Freiheit und Unsterblichkeit) unvermeidlich auf eben die Selbstwidersprüche und Fehlschlüsse getrieben, die sich am Kanon dogmatischer Lehrsysteme ablesen ließen. Zum anderen war es gerade die dogmatisch genannte Methodik wissenschaftlicher Forschung und Darstellung, die in ihrer schulgerechten Form streng logischer und prinzipiengeleiteter Erkenntnis auch in den Augen Kants als unverzichtbarer Bestandteil jeder wissenschaftlich verfahrenden Disziplin zu gelten hatte.

Mendelssohn (1785) 3.

Kant muß diese verfängliche Tendenz der zeitgenössischen Rezeption seines Werkes auch so wahrgenommen haben, spricht er doch in der Streitschrift gegen Eberhard ironisch von dem "Medusenhaupt der Kritik", das die rationale Psychologie und Theologie "zurückschrecken wollte". (E: AA VIII $199=$ WW III 311) 
Kants rigide antiskeptizistische Haltung machte so den Dogmatismus alternativelos. Obwohl Kant zwischen dem dogmatischen und skeptischen einen eigenständigen kritischen Weg anpeilte, drohte ihm doch seine Kritik unter der Hand zu einer Reform des Dogmatismus zu geraten. So jedenfalls lautete der Tenor der frühidealistischen KantKritik, der Kant selbst in der revidierten Fassung der $\mathrm{KrV}$ die Argumente lieferte.

Die Defizite des Dogmatismus sah Kant klar in der Naivität gebündelt, mit der Geltung und Reichweite seiner Behauptungen für beliebige Gebiete menschlichen Wissens und Glaubens ungerechtfertigt vorausgesetzt wurden. Abhilfe versprach sich Kant daher von der kritischen Musterung nun nicht nur des Objektbereichs unserer Erkenntnisbemühungen, sondern zunächst des funktionalen Komplexes menschlicher Erkenntnisse, den er als 'reine Vernunft' bezeichnete. Was Kant als seine 'Revolution der Denkart' ankündigte, machte den Dogmatismus in der eigentümlichen Wendung, die ihm sein Kritiker gab, jedoch erst reformfähig: "Dogmatismus ist also das dogmatische Verfahren der reinen Vernunft, ohne vorangehende Kritik ihres eigenen Vermögens." (BXXXV) Diese Beschreibung läßt zumindest den Schluß zu, daß der Dogmatismus, wenn er nur mit der gehörigen kritischen Propädeutik versehen wird, mit dem Anspruch einer wissenschaftlichen Metaphysik würde auftreten können. Nicht ohne patriotische Verve und um Fehlinterpretationen, die ihn gar auf eine bloß destruktive Haltung verpflichten wollten, zu entgehen, ließ sich Kant denn auch in der Vorrede zur zweiten Auflage der $K r V$ zu folgender Stellungnahme hinreißen:

"In der Ausführung also des Plans, den die Kritik vorschreibt, d.i. im künftigen System der Metaphysik, müssen wir dereinst der strengen Methode des berühmten Wolf, des größten unter allen dogmatischen Philosophen, folgen, der zuerst das Beispiel gab, (und durch dies Beispiel der Urheber des bisher noch nicht erloschenen Geistes der Gründlichkeit in Deutschland wurde,) wie [...] der sichere Gang einer Wissenschaft zu nehmen sei". (BXXXVI)

Was für Kant noch Präzisierung seiner kritischen Absichten sein mochte, mußte Schelling allerdings schon als einen untrüglichen Fingerzeig auf die verfänglichen Strukturen eines etablierten Kritizismus auffassen. Bereits 1797, als er noch an der Seite Fichtes den Geist des Kantianismus gegen eine allzu buchstabengetreue Auslegung verteidigte, machte Schelling treffsicher auf den nicht immer reflektierten, aber stets irritierenden Umstand aufmerksam, "daß Kant seine Philosophie, die allen Dogmatismus von Grund aus zerstören sollte, in der Sprache des Dogmatismus vortrug."3

Wo Kants Vernunftkritik der dogmatischen Terminologie verhaftet blieb, da lag der Verdacht nicht fern, daß die Kritik sich in wesentlichen Stücken nicht nur dem Instrumentarium des kritisierten Gegenstandes verdankte, sondern letztlich auch dessen Prämissen übernahm. In dieser ideologiekritischen Zuspitzung brachte Schelling dann im Todesjahr Kants seine frühen Bedenken gegen den Kritizismus auf den Punkt. Dessen Scheitern führte er darauf zurück, daß,

"indem er den Dogmatismus bekämpfte, durch die Art selbst, wie er den Kampf führte, beständig den Wahn voraussetzte und stehen ließ, daß jene dogmatische Art zu philosophiren eigentlich die wahre 
Philosophie seyn müßte", und weiter, "daß der Kriticismus zu seiner eigenen Existenz den Dogmatismus postuliren mußte". ${ }^{4}$

Kants Projekt der 'Grenzbestimmung' menschlicher Rationalität ließ sich von den Vorgaben der Dogmatismus-Kritik leiten und - in den Augen Schellings - täuschen. Zum Ansatz- und Revisionspunkt der frühidealistischen Kant-Kritik wurde so die mit der Option letztbegründeter Theorien unverträgliche Konsequenz allzu enger Grenzziehung, wonach gerade die fundamentalen Prinzipien, die bei der Gewinnung und Formulierung von Erkenntnis anzusetzen sind, selber nicht mehr erkennbar sein sollten.

Schelling und andere wurden daher nicht müde, die Prämissen der im ganzen akzeptierten Resultate der Kantischen Philosophie einzuklagen, doch war aus Kants Sicht die Frage nach den Prämissen genaugenommen falsch gestellt. Sowenig es Kant um die Dispensierung von Metaphysik ging, so zurückhaltend beurteilte er doch auch die Möglichkeit einer deskriptiven Metaphysik, deren immanente Schwierigkeiten, wie sich am Beispiel der MANW zeigen läßt, er weder im "Entwurf"5 von 1786 verschwieg noch in fortgesetzter, wenn auch (wie ihm aufging) vergeblicher Revision beseitigen konnte. Das kritizistische Unternehmen Kants war offensichtlich von bescheideneren Zielen geleitet, als ihm die Interpretationen seiner Kritiker zumuteten.

Mit Blick auf seine Dogmatismus-Kritik war es zunächst Kants Anliegen, für die Gewinnung vergleichbarer, auf ihren Wahrheitswert hin entscheidbarer Aussagen Kriterien zu formulieren, die es dem Wissenschaftler erlauben, zu "unterscheiden, ob gewisse Fragen in seinem Horizonte liegen, oder nicht" (A238 B297). Anders gesagt: "zu wissen, was man vernünftigerweise fragen solle" (A58 B82), war für Kant die naheliegende Konsequenz, die er aus seiner kritischen Beschäftigung mit metaphysischem Denken zog. Voraussetzungen, Formulierung und Perspektiven dieser dann 'kritisch' genannten Fragestellung sind Thema dieses Kapitels.

\subsection{Das Faktum der Erfahrung}

Kant spricht - erstaunlich genug - von der "Wirklichkeit der wissenschaftlichen Erkenntnisse a priori" (B128); darunter will er das Korpus der Erkenntnisse der 'reinen Mathematik' und 'allgemeinen Naturwissenschaft' verstehen. Beide Disziplinen scheint er ebenso wie das, was er Erfahrungserkenntnis nennt, als "Faktum" (B128) zu begreifen. Nicht weniger problematisch nimmt sich da seine Charakterisierung der Metaphysik aus, wenn er ganz unbefangen bekennt: "Irgend eine Metaphysik ist immer in der Welt gewesen, und wird auch wohl ferner, mit ihr aber auch eine Dialektik der reinen Vernunft, weil sie ihr natürlich ist, darin anzutreffen sein." (BXXXI)

Wer so redet, muß eine klare Vorstellung davon haben, welcher Sinn aus dieser paradoxen Grundstellung, in der sich Metaphysik manifestiert, zu gewinnen ist. Denn Metaphysik darauf deutet das Zitat - macht wie die Faktizität der genannten Erkenntnisse für Kant 
einen Teil der condition humaine aus. Und ohne Voraussetzung metaphysischer Prinzipien - so lautet Kants Hauptthese - verfügten wir gar nicht über die Erkenntnisse, die wir als 'wirklich' nur konstatieren können. Auf diese vertrackte Sachlage macht Kant mit einer Beobachtung (a) und einer These (b) aufmerksam, die, für sich genommen, gleichermaßen paradox sind.

(a) Schon zu Beginn der Vorrede der ersten Auflage der $K r V$ macht Kant seine Leser mit der Beobachtung bekannt, daß, wie er sich ausdrückt, die menschliche Vernunft "durch Fragen belästigt" werde, die sie zwar selbst aufwerfe, jedoch mit Recht weder abweisen noch eigentlich entscheiden könne. (AVII) Diese Fragen gelten der Existenz Gottes, des ersten Anfangs der Welt oder der Unsterblichkeit der Seele und damit Themen, die noch zur Zeit Kants in den Spezialdisziplinen der Metaphysik traktiert wurden. Nicht daß, aber wie wir uns Fragen diesen Typs stellen, deutet für Kant auf eine offenbar schon im Ansatz aporetische Verfassung des Verstandes. Danach führe jedes Aufsuchen von Bedingungen für etwas, das wir als Bedingtes auffassen, unweigerlich - "weil die Fragen niemals aufhören" (AVIII) - auf einen unendlichen Regreß stets bedingter Bedingungen. Dieser Regreß lasse sich dann, so scheint es, nur durch die Annahme vermeintlich 'erster' oder 'letzter' Gründe stoppen. Sieht man für das begriffliche Verstehen tatsächlich das logische Schließen nach Grund-Folge-Beziehungen als konstitutiv an, so können Gründe, die in einem Gedankengang notwendig als unbedingt vorausgesetzt werden, selbst nicht innerhalb dieses Gedankenganges erschlossen und daher auch nicht eigentlich begriffen werden.

Sollen die Lehrsätze der Metaphysik auf ihren Wahrheitsgehalt hin überprüfbar sein, so können die durch die im beschriebenen Sinne unlösbaren Fragen jedenfalls nicht derart Themen der Metaphysik sein, wie dies der Praxis der dogmatischen Systeme entsprach. Kant sieht die Kompetenz der kritisch gewendeten Metaphysik vielmehr darin, den epistemisch problematischen Status dieser metaphysischen Behauptungen zu bestimmen, und zwar für den Bereich der Metaphysik selber wie für diejenigen Wissenschaften, die sich (bewußt oder unbewußt) auf solche metaphysischen Sätze beziehen.

Kant hat denn auch aus der prinzipiellen Unentscheidbarkeit dieser Fragen nicht den Schluß gezogen, Metaphysik oder das Interesse an metaphysischen Fragen für obsolet zu erklären und zu verabschieden. Im Gegenteil, er hält diese metaphysischen Behauptungen geradezu für "unvermeidlich" (AX) immer dann, wenn wir, wie in theoretischen Wissenschaften, "urteilen wollen" und zwingender noch, wenn wir, wie in der Praxis zur Gewinnung moralisch qualifizierter Entscheidungen, "urteilen müssen". ${ }^{6}$ Unser fortgesetztes Interesse an Metaphysik sei daher "weniger Wißbegierde", als daß es einem "Bedürfnis der Vernunft"7, ja der "Naturanlage unserer Vernunft"8 entspreche. Diesem Bedürfnis zu entsagen - davon ist Kant überzeugt - sei daher ebensowenig möglich, "als daß wir, um nicht immer unreine Luft zu schöpfen, das Atemholen einmal lieber ganz und 
gar einstellen würden." ${ }^{9}$ Ob Kant Metaphysik als wesensmäßig im Menschen verankert ${ }^{10}$ oder eher als 'kulturelles' Ereignis (A850 B878) begreift, sei hier dahingestellt.

(b) Eher beiläufig bringt Kant, eine Formulierung Platners aufgreifend, diese im Hinblick auf seine Beobachtung (a) zunächst überraschende These an: "'In dem Wirklichen allein findet Unbegreiflichkeit statt.'"11 Soweit aus Kants Erläuterung dieses Zitats hervorgeht, bleibe uns das Wirkliche eben deshalb unbegreiflich, weil es nicht Begriff sei. Im Hinblick auf Erfahrung verständigten wir uns über das Wirkliche zwar auch mit Begriffen, doch seien diese Begriffe eben von dem, worüber wir uns verständigten, insofern unterschieden, als sie unser "eigenes Produkt" seien. Diese These muß erstaunen, wenn man sie vom Standpunkt der entfalteten transzendentalen Theorie der Erfahrung liest. Danach wissen wir von dem hier Wirklichkeit Genannten in der Erfahrung nur insofern, als wir diese Erfahrung, bezogen auf sog. Sinneseindrücke, nach einem festgelegten Kanon von Regeln so strukturiert haben, daß sie den Bedingungen physikalischer Beschreibung genügt. Was uns an den Dingen unbegreiflich bleibt, ist gerade nicht dasjenige, "was wir selbst in sie legen." (BXVIII) Daher klinge es "nur paradox", sei aber "nicht befremdlich", zu sagen: "in der Natur sei uns vieles unbegreiflich (z.B. das Zeugungsvermögen), wenn wir aber noch höher steigen und selbst über die Natur hinausgehen, so werde uns wieder alles begreiflich". ${ }^{12}$

Dieser durch das Bild des Auf- und Überstiegs angedeutete Perspektivenwechsel drückt genauer einen Wechsel im methodischen Zugang zu 'Wirklichkeit' aus und besagt den Wechsel vom alltäglichen, wenn auch naturwissenschaftlich beschreibbaren, zum transzendentalen Standpunkt. Letzterer wird mit der kritischen Fragestellung nach den Bedingungen der Möglichkeit von etwas, das ich nur vom Standpunkt des Alltagsbewußtseins kenne, eröffnet.

Diesen Gedanken hat Kant in einer Notiz zu seinem Handexemplar der $\mathrm{KrV}$ entwickelt. Zu A66 bemerkt Kant, daß in der "Einleitung" (A8) Erfahrung als der Inbegriff synthetischer Urteile a posteriori eingeführt, aber nicht weiter hinterfragt werde, "weil sie Factum ist." Nun behauptet Kant nicht, daß Erfahrung insofern Faktum sei, als sie für etwas stehe, daß bloß da und daher schlechterdings unbegreiflich sei. Kant interpretiert Erfahrung vielmehr als empirische Erkenntnis, die, in der Form eines Urteils, wahrheitsdifferente Aussagen über empirische Sachverhalte zuläßt. Erfahrung ist also Faktum, nur sofern sie Erkenntnis ist. Daher fährt Kant in der Notiz fort: "Jetzt läßt sich fragen, wie dieses Factum möglich sei, (Erfahrung besteht aus Urteilen, aber es fragt sich, ob diese empirischen Urteile nicht Urteile a priori voraussetzen) [...] Die Aufgabe ist: wie ist Erfahrung möglich?"13

Kants Beobachtung und These sprechen in einem doppelten Sinn von dem uns Unbegreiflichen. Zum einen ist die Idee des Unbedingten in theoretischen Kontexten nicht realisierbar, zum anderen ist das Faktum der Erfahrung für uns nur insofern hinterfragbar, als wir es als Erkenntnis interpretieren können. Das 'Wirkliche allein' im Sinne Plattners

\footnotetext{
$P:$ AA IV $367=$ WW III 245.

$P$ §57: AA IV $353=\mathrm{WW}$ III 228.

$P$ §5: AA IV $349=\mathrm{WW}$ III 223.

$P$ §5: AA IV $349=$ WW III 223.

Zitiert nach: Erdmann (1878) 21.
} 
bleibt uns unbegreiflich. Der Befund war für Kant Anlaß genug, sein Projekt anzugehen, die Strukturen und Reichweite menschlicher Rationalität im Sinne einer sich selbst aufklärenden Vernunft auszumessen. Dabei bringt Kant die Paradoxalität der Sachverhalte nicht einfach zum Verschwinden; er bezieht beide aufeinander und macht sie so zu Eckpfeilern seiner Theorie der menschlichen Erfahrungserkenntnis. Er kann so das Paradoxe als Prämissen einer Theorie integrieren, die er unter dem Titel "Kritik" im Sinne einer "Grenzbestimmung der Vernunft" vorgelegt hat. Damit verfolgt Kant genaugenommen das Projekt einer Wissenschaft, die - ausgreifend auf das Modell einer Theorie der Erkenntnis überhaupt und eingeschränkt auf die spezifischen Erkenntnisbedingungen des Menschen - eben "jene durch Kritik der Vernunft selbst allein mögliche Erkenntnis seiner Unwissenheit ist". (A758 B768) Der Kantischen Theorie dessen, 'was wir wissen können', geht voraus eine Theorie dessen, was 'uns notwendig unbekannt' bleiben muß. Kant hat das, was und wie uns etwas unbekannt bleiben muß, mit der Differenz der Begriffe 'Schranke' und 'Grenze' zu beschreiben versucht.

\subsection{Schranken und Grenzen}

Die Differenz von Schranke und Grenze verdeutlicht Kant am Beispiel der physischen und mathematischen Geographie. Kant geht davon aus, daß wir schon durch empirische Forschung und Beobachtung mühelos zu einer Erkenntnis dessen kommen, "was uns bei allem Wissen immer noch zu wissen übrigbleibt". (A758 B786) Hierzu führt er aus:

\footnotetext{
"Wenn ich mir die Erdfläche (dem sinnlichen Scheine gemäß) als einen Teller vorstelle, so kann ich nicht wissen, wie weit sie sich erstrecke. Aber das lehrt mich die Erfahrung: daß, wohin ich nur komme, ich immer einen Raum um mich sehe, dahin ich weiter fortgehen könnte; mithin erkenne ich Schranken meiner jedesmal wirklichen Erdkunde, aber nicht die Grenzen aller möglichen Erdbeschreibung." (A759 B787)
}

An dem, was wir wissen, unterscheidet Kant faktisches Wissen, das induktiv gewonnen und komparativ verallgemeinerbar ist, von prinzipiellem Wissen, das auf die Bestimmung des Verhältnisses dessen zielt, was wir faktisch wissen und nicht wissen können. Die Kenntnis faktischen Nichtwissens, Schranke genannt, ist zu trennen von der Kenntnis prinzipiellen Nichtwissens, Grenze genannt.

Die Erkenntnis der Schranke beruht auf etwas, 'das mich Erfahrung lehrt': "daß, wohin ich nur komme, ich immer einen Raum um mich sehe, dahin ich weiter fortgehen könnte". Kant behauptet nicht, daß uns dieser empirisch erschließbare Erfahrungsraum gleichsam als ein sich ins Unendliche erstreckendes Experimentierfeld gegeben sei. Seine Beschreibung des unendlichen Progresses in diesen Raum setzt vielmehr die Idee des Raumes als Kontinuum voraus, will sagen, der Progreß ist prinzipiell nicht abschlußfähig.

Davon unterschieden ist die Kenntnis von Grenzen, die ich empirisch im Fortschreiten nicht erwerben kann. So wie an Schranken stoße ich nicht an Grenzen, die nicht in den Bereich des a posteriori Erfahrbaren fallen. Eine Grenze ist offenbar gar kein Bestandteil desjenigen Raumes, den ich durchschreiten kann. Zu ihrer Kenntnis bedarf ich eines Perspektivenwechsels. Kant fährt fort: 
"Bin ich aber doch so weit gekommen, zu wissen, daß die Erde eine Kugel und ihre Fläche eine Kugelfläche sei, so kann ich auch aus einem kleinen Teil derselben, z.B. der Größe eines Grades, den Durchmesser, und durch diesen, die völlige Begrenzung der Erde, d.i. ihre Oberfläche, bestimmt und nach Prinzipien a priori erkennen; und ob ich gleich in Ansehung der Gegenstände, die diese Fläche enthalten mag, unwissend bin, so bin ich es doch nicht in Ansehung des Umfanges, der sie enthält, der Größe und Schranken derselben." (A759 B787)

Durch den Perspektivenwechsel gewinne ich nun Einblick in die Begrenzung des Untersuchungsfeldes, das ich dann mathematischen Prinzipien gemäß auszumessen in der Lage bin. Nach der Kantischen Sprachregelung setzt die Rede von der Grenze die Vorstellung eines Raumes voraus, der außerhalb eines 'bestimmten Platzes' liegt und denselben einschließt. ${ }^{14}$ Im Gegensatz zur Schranke verweist die Grenze nicht auf bloße Negationen dessen, was ich weiß, sondern auf etwas Positives in dem, was ich nicht wissen kann. ${ }^{15}$ Dieser Grenzbegriff steht damit für eine "Verknüpfung dessen, was wir kennen, mit dem, was wir nicht kennen und auch niemals kennen werden". ${ }^{16}$

Kant hat diese topographischen Metaphern für die Bestimmung der Funktionen unseres Erkenntnisvermögens, soweit es der 'Orientierung in der Welt' dient, genutzt.

\footnotetext{
"Aber die Begrenzung des Erfahrungsfeldes durch etwas, was ihr sonst unbekannt ist, ist doch eine Erkenntnis, die der Vernunft in diesem Standpunkte noch übrig bleibt, dadurch sie nicht innerhalb der Sinnenwelt beschlossen, auch nicht außerhalb derselben schwärmend, sondern so, wie es einer Kenntnis der Grenze zukommt, sich bloß auf das Verhältnis desjenigen, was außerhalb derselben liegt, zu dem, was innerhalb enthalten ist, einschränkt." 17
}

Die Begrenzung des Erfahrungsfeldes bedarf der transzendentalen Ideen, die als die klassischen Themen der Metaphysik eben jene 'Probleme der Vernunft' sind, die "durch ins Unendliche immer wiederkommende Fragen"18 aufgegeben, doch nicht gelöst werden können. Nach Kant formulieren diese Grenzbegriffe diejenigen Forschungsprinzipien, die als Einschränkung einen unendlichen Progreß der Forschung und zugleich als Grenzbestimmung die systematische Einheit der im Progreß erzielten Mannigfaltigkeit von Einzelergebnissen garantieren sollen. ${ }^{19}$ Mit der Differenzierung von Grenze und Schranke gelingt es Kant darüber hinaus, die Aufgabe der Metaphysik im Kanon der konkurrierenden wissenschaftlichen Disziplinen, Mathematik und Naturwissenschaft, zu bestimmen:

"In der Mathematik und Naturwissenschaft erkennt die menschliche Vernunft zwar Schranken, aber keine Grenzen, d.i. zwar, daß etwas außer ihr liege, wohin sie niemals gelangen kann, aber nicht, daß sie selbst in ihrem inneren Fortgange irgendwo vollendet sein werde. Die Erweiterung der Einsichten in der Mathematik und die Möglichkeit immer neuer Erfindungen geht ins Unendliche; ebenso die

14 P §57: AA IV $352=\mathrm{WW}$ III 227.

15 P §57: AA IV 354 = WW III 229. - Kant verdeutlicht seine Redeweise am Beispiel der Strukturmomente des euklidischen Raumes: "z.B. Fläche ist die Grenze des körperlichen Raumes, indessen doch selbst ein Raum, Linie ein Raum, der die Grenze der Fläche ist, Punkt die Grenze der Linie, aber doch noch immer ein Ort im Raume". Näheren Aufschluß über diese 'Strukturmomente der reinen Anschauung des Raumes' gibt Cramer (1985) 84-89. Vgl. 9.3.

16 P $\$ 7:$ AA IV $354=$ WW III 229.

17 P $\$ 9$ : AA IV $361=\mathrm{WW}$ III 237.

18 P 57: AA IV 353f. = WW III 228.

19 Diese Ideen werden, wie Kant in A771 B799 sagt, "bloß problematisch gedacht, um, in Beziehung auf sie (als heuristische Fiktionen), regulative Prinzipien des systematischen Verstandesgebrauchs im Felde der Erfahrung zu gründen." Als Ideen bestimmen sie insofern die Grenze, als sie gerade 'keine Gegenstände in irgendeiner Erfahrung' bezeichnen. 
Entdeckung neuer Natureigenschaften, neuer Kräfte und Gesetze durch fortgesetzte Erfahrung und Vereinigung derselben durch die Vernunft. [...] Allein Metaphysik führt uns in den dialektischen Versuchen der reinen Vernunft (die nicht willkürlich oder mutwilligerweise angefangen werden, sondern dazu die Natur der Vernunft selbst treibt) auf Grenzen; und die transzendentalen Ideen [...] dienen dazu, nicht allein uns wirklich die Grenzen des reinen Vernunftgebrauchs zu zeigen, sondern auch die Art, solche zu bestimmen". ${ }^{20}$

Das Projekt der 'Grenzbestimmung der reinen Vernunft' hat Kant mit der $K r V$ vorgelegt, die, als Propädeutik verstanden, denn auch "den Plan zum Ganzen einer Wissenschaft" (B109) entwirft. Die Ausführung und "Probe" (B110) dieses Plans gibt Kant mit den $M A N W$.

\subsection{Die Grenzen metaphysischer Anfangsgründe}

Wie ein Blick auf die Schlußpassage der $M A N W$ zeigt, steht die 'metaphysisch' genannte Körperlehre durchaus in der Perspektive der in der $\mathrm{KrV}$ projektierten Theorie der empirischen Erkenntnis von der Natur, deren Anwendung auf physikalische Theorien ohne Bezug auf das kritizistische Konzept der Grenzen menschlichen Wissens gar nicht zu leisten ist.

\footnotetext{
"Und so endigt sich die metaphysische Körperlehre mit dem Leeren und eben darum Unbegreiflichen, worin sie einerlei Schicksal mit allen übrigen Versuchen der Vernunft hat, wenn sie im Zurückgehen zu Prinzipien den ersten Gründen der Dinge nachstrebt, da, weil es ihre Natur so mit sich bringt, niemals etwas anders, als so fern es unter gegebenen Bedingungen bestimmt ist, zu begreifen, folglich sie weder beim Bedingten stehen bleiben, noch sich das Unbedingte faßlich machen kann, ihr, wenn Wißbegierde sie auffodert, das absolute Ganze aller Bedingungen zu fassen, nichts übrig bleibt, als von den Gegenständen auf sich selbst zurückzukehren, um, anstatt der letzten Grenze der Dinge, die letzte Grenze ihres eigenen sich selbst überlassenen Vermögens zu erforschen und zu bestimmen." ${ }^{21}$
}

Der resignative Grundton der Passage nimmt die Skepsis auf, die Kant mit vielen Naturwissenschaftlern seiner Zeit gegenüber metaphysischen Untersuchungen hinsichtlich der Diskrepanz von Erkenntnisanspruch und -leistung der Vernunft teilt. Den totalen Erkenntnisanspruch vermag die Vernunft dann nicht einzulösen, wenn es darum geht, die 'ersten Gründe' oder 'letzten Grenzen' der Dinge zu erforschen. Da sie, von Bedingtem ausgehend, immer nur auf Bedingungen schließen kann, ist ihr ein unvermittelter Zugang zu dem gleichwohl vorauszusetzenden Unbedingten abgeschnitten. Einen Ausweg sieht Kant in der Reflexion auf die Totalität der Bedingungen, die 'im Zurückgehen zu Prinzipien' nicht der Dinge, sondern der Erkenntnis der Dinge besteht. Mit dieser Wendung 'von den Gegenständen auf sich selbst' schließt Kant ausdrücklich an sein Unternehmen der 'Grenzbestimmung der Vernunft' an.

Worin genauer 'mit dem Leeren und eben darum Unbegreiflichen' das Ende der metaphysischen Körperlehre methodisch besiegelt wird, erläutert Kant auf den letzten Seiten der "Allgemeinen Anmerkung zur Phänomenologie". Hier entwickelt Kant unter den drei Hinsichten seiner 'metaphysischen Anfangsgründe' der Phoronomie, Dynamik und 
Mechanik denkbare Alternativen zu der physikalischen Annahme des Leeren, wie sie die mathematisch-mechanische Erklärungsart auf der Grundlage eines atomistischen Materiemodells erzwingt. Dagegen favorisiert Kant ein dynamistisches Modell zweier konfligierender Kräfte, das ihm erlauben soll, die Vorstellung leerer Räume wenn nicht aus der physikalischen Theorie zu beseitigen, so doch darin wenigstens überflüssig zu machen. Sieht man einmal von den spezifischen Implikationen physikalischer Modelle des Leeren ab, so ist der epistemische Status, den Kant jeweils den Alternativen zuschreibt, doch überraschend:

(a) Überzeugend scheint für Kant allenfalls sein Einwand gegen die Rede von der Existenz des leeren Raumes zu sein, in dem etwas Materielles als beweglich vorgestellt werden könne. Diese Idee des sog. absoluten Raumes sei eben als Abstraktion von empirisch bestimmbarer Materie genauer eine Bedingung, unter der etwas Materiellem Bewegung relativ zu anderem Materiellem zuzuschreiben möglich sei. Der leere Raum sei "also gar nichts, was zur Existenz der Dinge, sondern bloß zur Bestimmung der Begriffe gehört" und "sollte billig nicht ein leerer Raum genannt werden". ${ }^{22}$

(b) Kant räumt ein, daß die Annahme eines leeren, materie- und also kräftefreien Raumes ohne logischen Widerspruch zu denken möglich sei. Eine Chance, diese Annahme wenigstens als "physisch unmöglich" zu erweisen, sieht Kant dann für gegeben, wenn man die "Möglichkeit der Zusammensetzung einer Materie überhaupt [...] nur besser einsähe."23 Damit macht Kant selbst darauf aufmerksam, daß sein Modell, das die Zusammensetzung von Materie aus der Dualität konfligierender Kräfte erklären sollte, hier keine wirkliche Alternative zum Korpuskularmodell der Materie bietet. So tröstet er sich über diese Defizite mit Abwägungen zu Statusfragen hinweg. "Daß es [...] mit dieser Wegschaffung des leeren Raums ganz hypothetisch zugeht, darf niemanden befremden; geht es doch mit der Behauptung desselben nicht besser zu." ${ }^{24}$ Folgerichtig zieht sich Kant auf seine Dogmatismus-Kritik zurück, wobei die Kritik eben auch sein Kräftemodell, das zur Entscheidung steht, trifft: "Diejenige, welche diese Streitfrage dogmatisch zu entscheiden wagen, sie mögen es bejahend oder verneinend tun, stützen sich zuletzt auf lauter metaphysische Voraussetzungen [...] und es war wenigstens nötig, hier zu zeigen, daß diese über gedachte Aufgabe gar nicht entscheiden können." 25

(c) Gar keine Kompetenz billigt Kant der Metaphysik in der Frage zu, wie die Bewegung der Planeten zu erklären sei, ohne in der Himmelsmechanik auf die Annahme des 'gehäuften Leeren innerhalb dem Weltganzen' angewiesen zu sein. Hier muß sich Kant mit einem Hinweis auf das "schwer aufzuschließende Naturgeheimnis"26 zufrieden geben.

Kant hat die Defizite und Grauzonen seines 'Entwurfs' einer metaphysischen Körperlehre nicht geleugnet oder verschwiegen, sondern benannt. Sofern man diese korrigierenden Einschätzungen in der Forschung überhaupt wahrgenommen hat, wollte man darin

22 MANW: AA IV $563=\mathrm{WW}$ V 133.

MANW: AA IV $536=\mathrm{WW}$ V 134

MANW: AA IV $564=\mathrm{WW}$ V $134 \mathrm{f}$.

MANW: AA IV $564=$ WW V 135.

${ }^{26}$ MANW: AA IV $564=\mathrm{WW}$ V 135. 
Inkonsequenzen in der Durchführung des Programms sehen. ${ }^{27}$ Doch was seine Kritiker als Schwäche auslegen, scheint für Kant gerade die Stärke seiner Konzeption ausgemacht zu haben.

Als metaphysischer Theoretiker in Sachen Physik sieht sich Kant von seiten der Adressaten der $M A N W$, den sog. 'mathematischen Naturforschern', mit einem Wust an Vorurteilen gegen Metaphysik konfrontiert. Darunter sollen jene "den Wahn, sich Möglichkeiten nach Belieben auszudenken und mit Begriffen zu spielen"28, verstanden haben. Mathematik und Physik hingegen gelten als gelungene Beispiele wissenschaftlicher Disziplinen, deren Erfolge eben auch auf ihre Distanz zu und Abstinenz von Metaphysik zurückgeführt werden. Gemessen etwa an der Effizienz der Geometrie, die im Hinblick auf die Handlungspostulate "mit so Wenigem, was sie anderwärts hernimmt, so viel zu leisten vermag"29, nimmt sich die Ausbeute der Metaphysik, wie es scheint, kümmerlich aus: "Von der Metaphysik könnte man dagegen sagen: sie steht bestürzt, daß sie mit so Vielem, als ihr die reine Mathematik darbietet, doch nur so wenig ausrichten kann." ${ }^{30}$

Mit diesem vielzitierten Satz hat sich Kant weniger der Skepsis gegen metaphysische Theorien überhaupt anschließen wollen; seine Feststellung resultiert vielmehr aus der Hinterfragung der dogmatischen Lehrsysteme, deren unkritische Übernahme mathematischer Methodenideale und Verfahren metaphysische Denkansätze im ganzen ad absurdum geführt hat. Auf methodischem Gebiet gibt es zwischen Mathematik und Philosophie eben weder "Fortgang" noch "Annäherung", noch "Berührung". ${ }^{31}$ In der Fortführung des Zitats gibt Kant dem Gedanken denn auch eine eigentümliche Wendung, aus der sich die Aufgabe und Leistung dieser Konstellation von Disziplinen in wissenschaftssytematischer Perspektive bestimmen lassen:

"Indessen ist doch dieses Wenige etwas, das selbst die Mathematik in ihrer Anwendung auf Naturwissenschaft unumgänglich braucht, die sich also, da sie hier von der Metaphysik notwendig borgen muß, auch nicht schämen darf, sich mit ihr in Gemeinschaft sehen zu lassen." 32

Kant ist weit davon entfernt, zu meinen, Metaphysik sei (um im Bild zu bleiben) zum Schmuddelkind unter den Wissenschaften verkommen, in dessen Gemeinschaft zu zeigen der Mathematiker sich schämen müßte. Kant wird zeigen, daß die 'mathematischen Physiker' metaphysischer Prinzipien nicht nur notwendig bedürfen, sondern sie auch - und dies ist der Ansatzpunkt seiner Kritik an Newton - 'unbewußt' (und damit unkritisch) tatsächlich verwenden.

\footnotetext{
Tuschling (1971) 34-39.

MANW: AA IV $472=$ WW V 17.

MANW: AA IV 478f. = WW V 24.

MANW: AA IV $479=$ WW V 24.

$P$ §57: AA IV $353=\mathrm{WW}$ III 227.

32 MANW: AA IV 479 = WW V 24.
} 


\section{Was sind metaphysische Anfangsgründe der Naturwissenschaft?}

Die $M A N W$ sind seit den sechziger Jahren Gegenstand nicht unkontroverser, aber ertragreicher Forschungen gewesen. ${ }^{1}$ Ich beschränke mich hier auf eine knappe Skizze der Fragestellungen, die der Explikation des Problemtitels der metaphysischen Konstruktion im Lichte des in Kap.1 eingeschlagenen Weges der Kant-Interpretation dienen. Im Vordergrund stehen dabei die Fragen, (a) inwiefern die $M A N W$ als "Probe" (B110) der in der $K r V$ vorgelegten Propädeutik einer Wissenschaft von der Erfahrung aufgefaßt werden können; (b) was Kant unter der 'Zergliederung' des 'an sich empirischen Begriffs' der Materie im Verfahren der 'Durchführung' durch die Kategorien verstehen will. Beide Fragestellungen führen auf die Probleme, zu deren Beschreibung und nach wie vor ausstehenden Klärung man Kant die Einführung des Methodenbegriffs der metaphysischen Konstruktion unterstellt hat. Diesen Problemkomplex werde ich nach den Angaben, die Kant zu Zielsetzungen (2.1.), Theoriestatus (2.2.) und Verfahren (2.3.) metaphysischer Anfangsgründe macht, hier zunächst umreißen und dann in den folgenden Kapiteln durch eine Interpretation des Konstruktionsbegriffs in den $M A N W$ ausführlicher entwickeln.

\subsection{Zielsetzungen}

Die $M A N W$ gelten als ein inspirierendes Buch, das gleichwohl in Teilen historisch überholt ist und systematisch die mit dem ehrgeizigen Programm metaphysischer Anfangsgründe der empirischen Wissenschaft von der Natur (Physik) aufgeworfenen Schwierigkeiten auch nur auf dem Boden der Kantischen Transzendentalphilosophie nicht zufriedenstellend lösen konnte. Noch Kant selbst hat sich im Zuge der Revision der MANW im sog. OP zu dieser Einschätzung durchgerungen. ${ }^{2}$ Umgekehrt ist es gerade das Verdienst Kants, am Beispiel der Newtonschen Mechanik auf spezifische Begründungsprobleme der mathematisch verfahrenden Naturwissenschaft aufmerksam gemacht zu haben, indem er verdeckte metaphysische Gehalte physikalischer Aussagen freigelegt und daraus Rückschlüsse auf den epistemischen Geltungsanspruch solcher Aussagen gezogen hat. Ohne Rückbindung an ein kritisches Konzept von Empirizität hält Kant die Mathematisierung physikalischer Grundbegriffe für unzulässig. Denn dieses Vorgehen verleite den Wissenschaftler dazu, unbewußt solchen metaphysisch-dogmatischen

1 Es lassen sich zwei Schwerpunkte ausmachen: (a) die Stellung der MANW im System der Transzendentalphilosophie, die sich an dem Verhältnis der MANW zu KrV und OP festmacht; vgl. Forschungsbericht Stegmaier (1980) und Buchdahl (1984) mit Sammelband Butts (1986), Büchel (1987), Falkenburg (1987). Ungeklärt ist die Frage, welche Bedeutung den MANW für die Neuformulierung einzelner Theoreme der $K r V$ (B) zukommt. - (b) die frühe Rezeption der MANW: Tuschling (1971), Barnaby (1988), Carrier (1990).

2 Tuschling (1971) $119 \mathrm{f}$. 
Positionen aufzusitzen, die eine experimentelle Überprüfung der aufgestellten Gesetze und Hypothesen gerade unmöglich oder wertlos machen.

Wenn ich recht sehe, führen Kants Vorbehalte gegen eine angeblich metaphysikfreie Naturwissenschaft auf eine Fragestellung, die sich aus der modernen Axiomatisierung der Mechanik ergeben hat. Es geht darum, wie der Grundbegriff der Masse in einem axiomatischen Formalismus so definiert werden kann, daß der Bedeutungsgehalt des Begriffs nach seiner formalisierten Definition mit dem experimentell auf Beobachtung beruhenden Begriff in Übereinstimmung zu bringen ist. ${ }^{3}$ So seien im Unterschied zu einer rein hypothetisch-deduktiv verfahrenden Theorie, wie z.B. der axiomatisierten Geometrie, bei der Definition der Begriffe in der Mechanik, wie Jammer auffällig vage formuliert, "semantische Regeln oder Korrelationen mit der Erfahrung" zu berücksichtigen. ${ }^{4}$ Angesichts der bislang umstrittenen Versuche, etwa den Massenbegriff über das Maß bloß pragmatischer Setzungen hinaus überzeugend zu definieren ${ }^{5}$, hält es Jammer durchaus für gerechtfertigt, "wenn wir nicht auf genauen Definitionen der fundamentalen Begriffe bestehen, die der Aufstellung der Theorie vorausgehen, sondern eher den Bedeutungsgehalt dieser Begriffe durch den Aufbau der Theorie gewinnen." Gleichwohl gilt, daß das Definiendum "letzten Endes in seinen quantitativen Aspekten durch Rückgriff auf experimentelle Messungen determinierbar" sein müsse. ${ }^{6}$ Die Definition sage dann nicht, "was 'Masse' in Wirklichkeit sei", sondern ordnet "die quantitative Bestimmung gewissen experimentellen Verfahrensweisen" zu. ${ }^{7}$

Im Klartext heißt dies, daß die Bildung physikalischer Grundbegriffe auf Konventionen beruht. Ihre Bedeutung läßt sich erst aus dem Kontext der Ableitungsfunktionen erschließen, in denen sie nach axiomatischer Ordnung entwickelt und mit Hilfe physikalischer Größen und Einheiten beschrieben werden. Die empirische Rückkopplung der Begriffe ist dabei notwendig an die Anwendung quantifizierender Meßverfahren gebunden. Die (wie auch immer zu rechtfertigende) Mathematisierbarkeit der Begriffe gehört damit zu einer der Bedingungen, die geforderten 'Korrelationen mit der Erfahrung' herzustellen.

Diese knappe Problemskizze aus der Sicht des theoretischen Physikers soll verdeutlichen, welche Anforderungen die philosophische Grundlegung einer Erfahrungswissenschaft zu erfüllen hat. Demnach müssen die Ergebnisse experimenteller, d.h. empirisch ermittelter Messungen tatsächlich eine Entscheidung darüber zulassen, ob die betreffende Hypothese, wenigstens in dem einzelnen Testfall, als bestätigt oder widerlegt gelten kann. Es müssen also allgemeingültige Kriterien empirischer Wahrheit angebbar sein, die als solche bereits bei der Bildung der Hypothese und bei der Versuchsanordnung ihrer Überprüfung zu berücksichtigen sind. Diese Kriterien zu formulieren, fällt Kant zufolge jedoch in die Kompetenz nicht der jeweiligen Einzelwissenschaft, sondern der Metaphysik, sofern sie sich als Theorie der Erfahrung überhaupt versteht. Kant hat in der $\mathrm{KrV}$ eine Reihe solcher

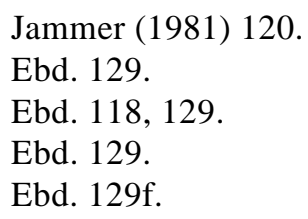


transzendental genannter Bedingungen objektivierbarer Erfahrungserkenntnis ausgearbeitet. Diese Bedingungen beschreiben umfassend die Gegenstandsstruktur von und unsere Zugangsart zu Erfahrung; sie sind dabei auf ein Konzept von "transzendentaler Wahrheit" hingeordnet, "die vor aller empirischen vorhergeht, und sie möglich macht." (A146 B185) Die MANW versuchen diese Vorgaben, die sich auf einen Begriff von Erfahrung überhaupt beziehen, für den spezifischen Bereich der physikalisch beschreibbaren Erfahrung umzusetzen - dies jedoch in so eigenwilliger Form, daß man darin gemeinhin einen Ausdruck des handgreiflichen Widerspruchs von Anspruch und Durchführung ihres Programms sieht. Nun sind die Angaben, die Kant selbst zu Programm, Theoriestatus und Darstellungsform der MANW macht, zugegebenermaßen ambivalent und verwirrend. Zumindest spricht daraus eine gewisse Unentschiedenheit, die, vorsichtig formuliert, Interpretationsspielräume offenhält und, so ist mein Eindruck, im Sinne des von Jammer gewiesenen Auswegs durchaus offenhalten sollte.

Schon mit der Wahl des Titels gibt Kant den Lesern zu verstehen, daß "Anfangsgründe" eben "nur Annäherung zum System, nicht dieses selbst" darstellten. ${ }^{8}$ Diesem Anspruch entspricht auch der Entwurfscharakter der $M A N W$, der sich an der Form der Darstellung nach 'mathematischer Methode' manifestiert, die Kant "wenn gleich nicht mit aller Strenge befolgt [...], dennoch nachgeahmt" habe. ${ }^{9}$ Es scheint, als habe Kant mit der Präsentation seiner Ergebnisse nach der 'dogmatischen Lehrart' den Standards wissenschaftlicher Prosa genügen wollen. ${ }^{10}$ Man hat dies als "verfehlten Ehrgeiz" und "in der Darstellungsform höchst unzureichend" kritisiert, weil die nachweisbare Geltung einzelner Behauptungen ihrer vorgeblichen Kennzeichnung (z.B. als 'Erklärung' oder 'Beweis') in der Regel gar nicht genügten. ${ }^{11}$ Dem vorläufigen Charakter der Abhandlung scheint indes Kants Anspruch zu widersprechen, "diese metaphysische Körperlehre so weit, als sie sich immer nur erstreckt, vollständig erschöpft [...] zu haben." Daß Kant wie "in allem, was Metaphysik heißt", so auch für die MANW "absolute Vollständigkeit"12 reklamiert, glaubt er durch deren enge Anbindung an die mit der $K r V$ vorgegebenen Propädeutik jeder wissenschaftlichen Systematik, als deren "Probe" (B110) er die MANW ja ausgibt, eingelöst zu haben.

Mit den $M A N W$ verbindet Kant die Absicht, Metaphysik nicht in Konkurrenz, sondern als Komplement zu einer mathematisch verfahrenden Naturwissenschaft Newtonscher Prägung zu etablieren. Dabei ging Kant von der Voraussetzung aus, daß die zeitgenössische Naturwissenschaft sich nur scheinbar und überdies mit verfehltem Anspruch von der Last des scholastischen Überbaus der traditionellen Metaphysik emanzipiert hatte. Dem methodischen Postulat einer vollständigen Systematik folgend und dem kritischen Anspruch der Falsifizierbarkeit wissenschaftlicher Aussagen verpflichtet, sollte die kritisch gewendete Metaphysik nun für die Bildung und Reglementierung

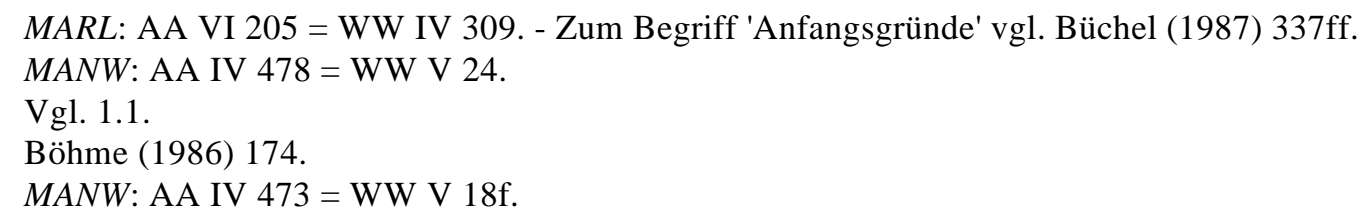


naturwissenschaftlicher Hypothesen fruchtbar gemacht werden. So versteht Kant Naturwissenschaft geradezu als "angewandte Metaphysik". ${ }^{13}$

Das Unternehmen, das Kant hier vorschwebt, war überaus anspruchsvoll, ja heikel. Man erinnere sich: Die Reform der Metaphysik als Wissenschaft bedurfte eines Konzeptes, das einerseits das in den Augen Kants unbestreitbare Bedürfnis nach Metaphysik als "metaphysica naturalis" (B21) und ihre Inanspruchnahme als "Grundwissenschaft" (BXXIV) versöhnen konnte und andererseits eine geschlossene Systematik vorgab, die die Forschungsergebnisse der so innovativen und stetig expandierenden Naturwissenschaften jederzeit zu integrieren erlaubte.

An diesen Vorgaben orientiert, drohte die Ausführung des Programms der MANW von vornherein an einem Zielkonflikt zu scheitern: Die Instrumentalisierung der MANW als "Realisierung der Transzendentalphilosophie" und zugleich ihre Integrierung als "besonderen Grundteil" der allgemeinen Physik ${ }^{14}$ versuchte Kant formal durch offene Argumentation bei geschlossener Darstellungsweise umzusetzen. Dieses Vorhaben ist Kant nach Meinung der Interpreten nicht geglückt. Die unklare Gemengelage von Argumentation und Darstellungsform verleitet Tuschling gar zu der These, Kant habe mit den $M A N W$, die "sowohl metaphysische wie mathematische Erkenntnis sein wollen, sich also selbst widersprechen, [...] ein Unding" kreiert. ${ }^{15}$ Die Bildung des Problemtitels 'metaphysische Konstruktion' und die Vielzahl divergierender Deutungen, die man diesem vermeintlich genuinen Methodenbegriff der $M A N W$ gegeben hat, stehen für den Versuch, die Inkonsequenzen der Schrift entweder einzuebnen oder wenigstens auf einen Begriff zu bringen.

Kants Bemerkung in der Vorrede der MANW zum "Titel dieses Werks, welches eigentlich[!] die Grundsätze der Körperlehre enthält", macht stutzig, weist sie doch schon in der Formulierung auf eine Reihe von Komplikationen, etwa den Ausschluß der Psychologie und Chemie "von dem Range einer eigentlich[!] so zu nennenden Naturwissenschaft"16. Schon die für Kants Euvre einzigartige Häufung 'uneigentlicher' Ausdrucksweisen in der Vorrede ('eigentlich', 'an sich empirisch') deuten die Art von Schwierigkeiten an, die Kant mit der Umsetzung der doppelten Zielsetzung der Abhandlung hat: (a) Als besondere Metaphysik sollen die MANW der allgemeinen Metaphysik "Beispiele (Fälle in concreto)" oder "Anschauungen" liefern, auf die bezogen sich erst die "Begriffe und Lehrsätze" der allgemeinen Metaphysik - darunter sei "eigentlich die Transzendentalphilosophie" zu verstehen - "realisieren" lassen. ${ }^{17}$ Mit dem Fehlen dieses Systemteils wäre nicht überprüfbar, ob den transzendentalen Begriffen überhaupt "objektive Realität, d.i. Bedeutung und Wahrheit"18 zukommt oder nicht. Die $M A N W$ sind damit ein integraler Baustein seiner Theorie der Metaphysik als Theorie der Erfahrung, ohne den Metaphysik dem wissenschaftlichen Kriterium wahrheitsdifferenter

$M A N W:$ AA IV $482=$ WW V 27

MANW: AA IV $478=$ WW V 24

Tuschling (1971) 115.

MANW: AA IV $471=\mathrm{WW}$ V $15 \mathrm{f}$.

$M A N W:$ AA IV $478=\mathrm{WW}$ V 23f.

$18 M A N W$ : AA IV $478=$ WW V 23. 
Aussagen nicht genügen würde. (b) Den Adressaten der $M A N W$, den mathematischen Naturforschern, schlägt Kant vor, "den metaphysischen Teil [...] in ihrer allgemeinen Physik als einen besonderen Grundteil zu behandeln und mit der mathematischen Bewegungslehre in Vereinigung zu bringen." ${ }^{19}$

Zum einen geht es Kant um die Besonderung metaphysischer Prinzipien "nicht allein von der Physik, welche empirische Prinzipien braucht, sondern selbst von den rationalen Prämissen derselben, die den Gebrauch der Mathematik in ihr betreffen". Zum anderen muß dieser besondere Grundteil einer allgemeinen Physik sich eben am Bestand der zeitgenössischen Naturwissenschaft orientieren, die Kant im Konzept einer "durchgängig" reinen oder angewandten Bewegungslehre vorgefunden hat. ${ }^{20}$

Dieses Konzept der Naturwissenschaft als 'allgemeiner Bewegungslehre' ist metaphysisch nicht deduzierbar und daher einer metaphysischen Letztbegründung auch gar nicht fähig. Es ist eben darum auch nicht "ein von der Transzendentalphilosophie nicht bewältigtes Residuum der Tradition"21; vielmehr besteht in der Anerkennung der Tatsache oder 'Wirklichkeit' der Naturwissenschaft gerade die Voraussetzung der transzendentalen 'Nachfrage' nach der Beziehung von 'Zugangsart' zu und 'Gegenstandsstruktur' von Erfahrung $^{22}$, d.h. nach den rezeptiven und konstitutiven Bedingungen unseres Erkenntnisvermögens, die uns das, wovon wir im Sinne Kants Erfahrung machen, als objektiv erkennbar verstehen lassen. Erweist sich einerseits die mathematisch verfahrende Naturwissenschaft als von einem übergeordneten metaphysischen Konzept ihres Untersuchungsgegenstandes abhängig, so bezieht andererseits Metaphysik ihre Legitimität gerade aus der Hinordnung auf diese Erfahrungswissenschaft, durch deren "Beispiele (Fälle in concreto)" ihre Grundbegriffe und -sätze erst 'realisiert' werden. Sofern sich die $M A N W$ nun mit den durch den Begriff der Materie bezeichneten empirischen Sachverhalten beschäftigen, die als Faktum metaphysisch nicht herleitbar, sondern kontingenterweise (nämlich am Kanon der zeitgenössischen Naturwissenschaft orientiert) vorgegeben sind, formulieren sie selber nur, was die physikalisch objektivierbaren Bestimmungen von Materie betrifft, eingestandenermaßen Prinzipien, die zur Bildung von Aussagen mit hypothetischer Geltung taugen. ${ }^{23}$ Kant beansprucht zwar, daß diese Hypothesen auf der Grundlage seiner 'metaphysisch-dynamischen Erklärungsart' unter wahrheitstheoretischem Aspekt besser begründbar sind als die entsprechenden nach der 'mathematisch-mechanischen Erklärungsart' gebildeten Hypothesen. Er beansprucht aber nicht, die Universalität der in den objektiven Erklärungen gegebenen Bestimmungen des Begriffs der Materie nachzuweisen. Denn nach Kants eigenen methodologischen Vorgaben dienen Definitionen am Anfang einer philosophischen Theorie nur "zum bloßen Versuche" (A730 B758) und als "Annäherungen" (A731* B759*), deren Wahrheit jedoch überprüfbar sein muß. Als 'besonderer Grundteil' der theoretischen Physik, die einen Teil der Grundlagenreflexion der Metaphysik überantwortet hat, sind solche Definitionen -

$19 M A N W:$ AA IV $478=$ WW V 24.

20 MANW: AA IV $477=$ WW V 22.

21 Hoppe (1969) 64.

22 Vgl. Böhme (1986) 182.

23 Vgl. 1.4. 
wenngleich philosophisch immer unvollständig - als axiomatische Setzungen nicht nur legitim, sondern unabdingbar, sofern sie nur den Erfolg empirischer Forschung gewährleisten.

Das auf den ersten Blick nach Form und Gehalt widersprüchliche Erscheinungsbild der $M A N W$ ist geradezu ein beredter Ausdruck der doppelten Zielsetzung, die auf einen Konflikt ihrer methodischen Prinzipien führt, den man gemeinhin mit dem Ausdruck 'metaphysische Konstruktion' bezeichnen will. Die hier exponierte doppelte Zielsetzung läßt sich auch an Theoriestatus (2.2) und Verfahren (2.3.) der MANW aufweisen.

\subsection{Theoriestatus}

Die MANW nennt Kant "eine wirkliche Metaphysik der körperlichen Natur" ${ }^{24}$ oder "metaphysische Körperlehre"25 oder "besondere metaphysische Naturwissenschaft"26 oder "metaphysische Anfangsgründe der Körperlehre"27 oder "eine abgesonderte Metaphysik der körperlichen Natur"28. Nach den topologischen Vorgaben der traditionellen Metaphysik sollen die MANW als physica rationalis den ersten der beiden Teilbereiche der rationalen Physiologie, worunter Physik und Psychologie fallen, einnehmen. (A846f. B874f.)

"Die Metaphysik der körperlichen Natur heißt Physik, aber, weil sie nur die Prinzipien ihrer Erkenntnis a priori enthalten soll, rationale Physik." (A846 B874) Mithin formulieren die MANW Prinzipien einer Physik genannten Disziplin, die (als Physik oder Naturwissenschaft) noch eines rationalen Teiles bedarf, der die Apriorität 'ihrer Erkenntnis' begründet. Physik ist weder Metaphysik noch darauf reduzierbar; Physik bedarf jedoch der 'rationale Physik' genannten Instanz, die, als 'metaphysische Anfangsgründe' angekündigt, die 'Prinzipien ihrer Erkenntnis a priori' angibt.

Rationale Erkenntnis ist Kant zufolge cognitio ex principiis (A836 B864) und als solche sowohl philosophische wie mathematische Erkenntnis, die sich nach ihrer spezifischen Erkenntnisart als Erkenntnis aus Begriffen und als Erkenntnis aus der Konstruktion von Begriffen unterscheiden. Als rationale Physik formulieren die MANW Erkenntnisse aus Begriffen. Die $M A N W$ will Kant strikt unterschieden wissen von Newtons "mathem. Grundlehren der Nat. Wiss." ${ }^{29}$, die schon nach dem Titel auf mathematischen Prinzipien beruhen sollen. Aus Kants Sicht unterläuft Newton wie "alle[n] Naturphilosophen, welche in ihrem Geschäfte mathematisch verfahren wollten", 'unbewußt' die "Vermengung" metaphysischer und mathematischer und damit eben 'ungleichartiger' Prinzipien. ${ }^{30}$ Die $M A N W$ sollen sich damit auch von dem unterscheiden, "was man gemeiniglich physica generalis nennt, und mehr Mathematik, als Philosophie der Natur ist" (A847* B875*),

\footnotetext{
MANW: AA IV $472=\mathrm{WW}$ V 17.

$M A N W:$ AA IV 473, $564=$ WW V 19, 135.

$M A N W$ : AA IV $470=\mathrm{WW}$ V 14

$M A N W$ : AA IV $477=\mathrm{WW}$ V 22.

$M A N W$ : AA IV $478=\mathrm{WW}$ V 23

$M A N W:$ AA IV $478=\mathrm{WW}$ V 24

$M A N W$ : AA IV 472f. $=\mathrm{WW}$ V $17 \mathrm{f}$.
} 
oder, wie es mißverständlich genug in der Vorrede der MANW heißt, von der "(physica generalis), wo metaphysische und mathematische Konstruktionen durch einander zu laufen pflegen". ${ }^{31}$

Kant will in dieser "Propädeutik der Naturlehre, die unter dem Titel der allgemeinen Naturwissenschaft [physica generalis] vor aller Physik (die auf empirische Prinzipien gegründet ist) vorhergeht", mit den $M A N W$ einen eigenständigen metaphysischen Teil isolieren, der eben "bloß diskursive Grundsätze (aus Begriffen), welche den philosophischen Teil der reinen Naturerkenntnis ausmachen", enthält. ${ }^{32}$ Erhebliche Irritationen in der Forschung hat freilich Kants Feststellung ausgelöst, nach der er in diesem philosophischen Teil der reinen Naturerkenntnis "doch auch manches" ausmachen will,

"was nicht ganz rein und von Erfahrungsquellen unabhängig ist: als der Begriff der Bewegung, der Undurchdringlichkeit (worauf der empirische Begriff der Materie beruht), der Trägheit u.a.m., welche es verhindern, daß sie nicht ganz reine Naturwissenschaft heißen kann". ${ }^{33}$

Hiermit benennt Kant genau diejenigen 'metaphysischen Prinzipien',

"welche den Begriff ihres eigentlichen Gegenstandes, nämlich der Materie, a priori zur Anwendung auf äußere Erfahrung tauglich machen, als des Begriffs der Bewegung, der Erfüllung des Raums, der Trägheit, u.s.w." ${ }^{34}$.

Offenbar sind vom reinen Teil der Naturwissenschaft mit dem metaphysischen Teil gerade die 'nicht ganz reinen' Begriffe abzusondern, und es sind diese Begriffe und - so sei hier im Vorgriff behauptet - "mit ihnen zugleich die Prinzipien der Konstruktion dieser Begriffe"35, die die $M A N W$ als ein System darstellen. Daß die Begriffe 'nicht ganz rein und von Erfahrungsquellen unabhängig sind', soll dabei nicht der Apriorität metaphysischer Erkenntnisse widersprechen. Damit ist für die $M A N W$ auf eine besondere Gemengelage von Sach- und Darstellungsproblemen verwiesen, die ich vorläufig so zusammenfassen möchte: Kant kennzeichnet metaphysische Erkenntnis als diskursiv, apriorisch und 'nicht ganz rein', sofern sie auf das Faktum der Gegebenheit empirischer Sachverhalte bezogen wird. Zwar ist die metaphysische Art von Erkenntnis von der mathematischen Art spezifisch unterschieden, doch ermöglicht sie gerade die Quantifizierbarkeit der empirischen Sachverhalte und damit einerseits die naturwissenschaftlich beschreibbare Erfahrung und andererseits zugleich den Nachweis empirischer Wahrheit.

Der hier bloß skizzierte Problemgehalt der $M A N W$ drückt sich auch in der komplexen Formation von Metaphysik, Mathematik und Naturwissenschaft aus, die so, wie sie Kant in der Vorrede der $M A N W$ arrangiert, zunächst bizarr anmutet, sich jedoch präzise beschreiben läßt:

"Also mag zwar eine reine Philosophie der Natur überhaupt, d.i. diejenige, die nur das, was den Begriff einer Natur im allgemeinen ausmacht, untersucht, auch ohne Mathematik möglich sein, aber eine reine Naturlehre über bestimmte Naturdinge (Körperlehre und Seelenlehre) ist nur vermittelst der

\footnotetext{
31 MANW: AA IV $473=\mathrm{WW}$ V 18.

$P \S 15:$ AA IV $295=\mathrm{WW}$ III 160.

Ebd.

34 MANW: AA IV $472=\mathrm{WW}$ V 17

35 MANW: AA IV $473=\mathrm{WW}$ V 18.
} 
Mathematik möglich, und, da in jeder Naturlehre nur so viel eigentliche Wissenschaft angetroffen wird, als sich darin Erkenntnis a priori befindet, so wird Naturlehre nur so viel eigentliche Wissenschaft enthalten, als Mathematik in ihr angewandt werden kann. ${ }^{36 "}$

Was Naturlehre zu einer Wissenschaft im strengen Sinne macht, ist offenbar die mögliche Anwendung von Mathematik auf den spezifischen Gegenstandsbereich dieser Disziplin. Im ersten Teil der Satzperiode sagt Kant über den je eigenen Gegenstandsbereich der Disziplinen aus, daß "reine Philosophie der Natur überhaupt" auch ohne und "reine Naturlehre über bestimmte Naturdinge" nur vermittelst der Mathematik, wie es heißt, 'möglich' seien. Die Anwendung von Mathematik ist für den Fall der reinen Philosophie fakultativ, für den Fall der reinen Naturlehre konstitutiv gesetzt. Im zweiten Teil der Periode scheint Kant unter Ausblendung der 'reinen Philosophie' nur noch von Naturlehre zu sprechen. Dabei versucht er zu bestimmen, in welchem Zusammenhang der Status "jeder Naturlehre" als "eigentlicher Wissenschaft" einerseits und das Vorliegen apriorischer Erkenntnis sowie die Anwendung der Mathematik 'in ihr' andererseits stehen.

So behauptet Kant wohl, daß Naturlehre nur in dem Maße eigentliche Wissenschaft sei, als sie apriorische Erkenntnis formuliere. Kant behauptet jedoch nicht, daß dieser Typ apriorischer Erkenntnis schon identisch sei mit der Formulierung mathematischer Aussagen über den Gegenstandsbereich der Naturlehre. Genauer muß gesagt werden, daß Naturlehre eigentliche Wissenschaft ist nur insofern, 'als Mathematik in ihr angewandt werden kann', diese mögliche Anwendung jedoch unter der Voraussetzung steht, daß 'sich darin Erkenntnis a priori befindet'. Der Anwendung der Mathematik auf bestimmte Naturdinge, d.h. die mathematisch exakte Beschreibung empirisch erhebbarer Daten über Natur, geht voraus eine metaphysische Theorie dessen, was als Naturding Dasein hat und in einem System physikalischer Größen bestimmbar ist. Dies besagt die Kantische These, daß 'eigentlich so zu nennende Naturwissenschaft' zuerst 'Metaphysik der Natur' voraussetze. ${ }^{37}$ Metaphysik der Natur präformiert den Gegenstandsbereich der Naturlehre so, daß die Anwendung quantifizierender Meßmethoden so etwas wie 'eigentliche Wissenschaft' indiziert, nicht jedoch allein ermöglicht.

Erstaunlich ist freilich, daß eine solche Konstituierung des Gegenstandsbereichs 'bestimmte Naturdinge' die Ausarbeitung der Naturlehre zur Wissenschaft nicht nur ermöglichen, sondern zwingend machen soll. Kant behauptet jedenfalls, "daß nach Foderungen der Vernunft, jede Naturlehre zuletzt auf Naturwissenschaft hinausgehen und darin sich endigen müsse". ${ }^{38} \mathrm{Im}$ folgenden bleibt zu untersuchen, wie die Konstituierung des Gegenstandsbereichs der Naturlehre durch eine Metaphysik der Natur und metaphysische Körperlehre und die damit einhergehende Forderung, Naturlehre in Naturwissenschaft zu überführen, zusammenhängen. 


\subsection{Verfahren}

Die Charakterisierung der $M A N W$ als "Probe" (B110) im Sinne der 'Realisierung eigentlich der Transzendentalphilosophie' läßt sich in zweifacher Hinsicht entwickeln: (a) als Index des in der Forschung als 'Anwendungsproblem' umschriebenen Sachverhalts, wonach die Besonderung des Gegenstandsbereichs der MANW von 'Natur überhaupt' zu 'besonderen Naturdingen' mit der Anwendung der transzendentalen Prinzipien auf die Gattung der Gegenstände der äußeren Sinne (Materie) vollzogen wird. Es ist gerade dieses Anwendungsproblem, als dessen Explikation man Plaaß' Interpretation des Begriffs der metaphysischen Konstruktion verstanden hat. ${ }^{39}$ (b) als Ausweis der Systematizität in Ordnung und Anzahl aller Momente einer Metaphysik der Natur.

(a) Kant bezeichnet die $M A N W$ in B110 als "Probe" der in der $K r V$ entwickelten Propädeutik "einer vorhabenden spekulativen Wissenschaft". Man hat dies als Index des als 'Anwendungsproblem' beschriebenen Sachverhalts aufgefaßt, wonach in den MANW die Besonderung des Gegenstandsbereichs der Natur überhaupt zu dem der besonderen Naturdinge mit der 'Anwendung' transzendentaler Prinzipien des allgemeinen oder transzendentalen Teils der Metaphysik auf die eine Gattung der Gegenstände der äußeren Sinne einhergeht. ${ }^{40}$ Diese Gattung wird durch den "abgesonderten (obzwar an sich empirischen) Begriff" 41 der Materie repräsentiert und bildet damit das Themengebiet der "besonderen metaphysischen Naturwissenschaft"42. Seit Plaaß ist man sich darin einig, daß die durch das 'Anwendungsproblem' indizierte Besonderung der Gegenstandsbereiche nicht im Sinne der analytischen Herleitung der besonderen Naturgesetze aus den in der $K r V$ formulierten transzendentalen Gesetzen einer Natur überhaupt verstanden werden kann. ${ }^{43}$ Dazu führt Kant in B165 aus: "Besondere Gesetze, weil sie empirisch bestimmte Erscheinungen betreffen, können davon [Gesetze, auf denen eine Natur überhaupt, als Gesetzmäßigkeiten der Erscheinungen in Raum und Zeit, beruht] nicht vollständig abgeleitet werden, ob sie gleich alle insgesamt unter jenen stehen." Die Begriffe und Grundsätze, die die Anwendung des Begriffs der Materie in der Physik ermöglichen sollen (Bewegung, Undurchdringlichkeit und Trägheit), sind also nicht unbezüglich auf, aber auch nicht vollständig aus besagten transzendentalen Prinzipien herleitbar. Sie sind daher auch nicht empirisch, sondern sollen gerade die Anwendung auf Empirie erklären und sind somit konstitutiv für das, was durch den von Kant so genannten 'an sich empirischen Begriff' der Materie bezeichnet wird. Im Unterschied zu transzendentalen Prinzipien gehen metaphysische Prinzipien auf Dinge, die schon als mögliche Objekte unserer Erkenntnis bestimmbar sind; d.h. der besondere Bereich, von dem ein empirischer Begriff gegeben ist, das Gattungsmerkmal von Gegenständen möglicher Erfahrung schon erfüllt. ${ }^{44}$

(b) Wenn Kant von den MANW als "Probe" der $K r V$ spricht, scheint er jedoch (noch) einen anderen als den hier durch das 'Anwendungsproblem' charakterisierten Sachverhalt vor

39 Vgl. Cramer (1985) $125 \mathrm{f}$.

40 MANW: AA IV 469f. = WW V 14.

41 MANW: AA IV $472=\mathrm{WW}$ V 16.

42 MANW: AA IV $470=\mathrm{WW}$ V 14.

43 Plaaß (1965) 115f., Cramer (1985) $125 f$.

44 Vgl. KU XXIXf. mit Mech.Ls.3; dazu Plaaß (1965) und Cramer (1985) $125 f$. 
Augen zu haben. Im $\S 11$ der B-Auflage der $K r V$ erläutert Kant, inwieweit eine anhand der "Tafel der Kategorien" dargestellte 'systematische Topik' (A83 B109) "vielleicht erhebliche Folgen in Ansehung der wissenschaftlichen Form aller Vernunfterkenntnisse" (B109) habe:

\begin{abstract}
"Denn daß diese Tafel im theoretischen Teile der Philosophie ungemein dienlich, ja unentbehrlich sei, den Plan zum Ganzen einer Wissenschaft, sofern sie auf Begriffen a priori beruht, vollständig zu entwerfen und sie mathematisch nach bestimmten Prinzipien abzuteilen; erhellt schon von selbst daraus, daß gedachte Tafel alle Elementarbegriffe des Verstandes vollständig, ja selbst die Form eines Systems derselben im menschlichen Verstande enthält, folglich auf alle Momente einer vorhabenden spekulativen Wissenschaft, ja sogar ihre Ordnung, Anweisung gibt, wie ich denn auch davon anderwärts* [* Metaphys. Anfangsgr. der Naturwissensch.] eine Probe gegeben habe." (B109f.)
\end{abstract}

Mit dieser Beschreibung der Tafel als systematischer Topik löst Kant offenbar seine in B27 gemachte Vorgabe ein, wonach die Transzendentalphilosophie "die Idee einer Wissenschaft" sei, "wozu die Kritik der reinen Vernunft den ganzen Plan architektonisch, d.i. aus Prinzipien, entwerfen soll, mit vollständiger Gewährleistung der Vollständigkeit und Sicherheit aller Stücke, die dieses Gebäude ausmachen."

Kant hat in der B-Auflage. der $K r V$ immer wieder betont, daß die 'Kritik' nur der "Traktat von der Methode, nicht das System selbst" sei (BXXII). Das System "würde Transzendental-Philosophie heißen. Diese ist aber wiederum für den Anfang noch zuviel." (B25) Daß Kant das 'noch' erst in der B-Auflage. einfügt, kann so verstanden werden, daß er mit den $M A N W$ einen ersten Schritt - "nur Annäherung zum System, nicht dieses selbst"45 zur Realisierung "eigentlich der Transzendentalphilosophie"46 getan hat. Entsprechend schweigt er sich in der $K r V$ über die "eigentümliche Methode der Transzendentalphilosophie" noch aus. (A738 B766)

So ist der Interpret mit dem eigenartigen Phänomen konfrontiert, daß Kant zwar System und Methode der Transzendentalphilosophie nicht ausführt, gleichwohl mit transzendentalen Prinzipien und Deduktionen im Kontext einer "Propädeutik" zu eben der Realisierung dieser Wissenschaft operiert. Die Konsequenz, mit der Kant die Funktion seiner wissenschaftlichen Propädeutik gerade in der B-Auflage verficht, fordert zu einer weitergehenden Interpretation auf als diejenige, die Kants Systematizitätsanspruch als Rigorismus oder bloßen Begriffsmanierismus abtut. Es ist gerade das Kriterium der Vollständigkeit aller Bestimmungen, das für Kant integraler Bestandteil dessen ist, womit die eigentümliche Leistung der Metaphysik vor der der Naturwissenschaft und Mathematik beschrieben werden kann. ${ }^{47}$ Für Kant stellt es geradezu "einen besonderen Reiz" dar, daß in allem, was Metaphysik heißt, die absolute Vollständigkeit der Wissenschaften gehofft werden kann [...], mithin eben so, wie in der Metaphysik der Natur überhaupt, also auch hier die Vollständigkeit der Metaphysik der körperlichen Natur zuversichtlich erwartet werden kann". Kant visiert dabei sogar "eine bestimmte Zahl von Erkenntnissen" an. ${ }^{48}$ Damit avanciert die Tafel der Kategorien zur Relaisstation zwischen allgemeiner und spezieller Metaphysik; unmißverständlich heißt es dazu in der Vorrede der MANW: "Das

Vgl. 1.3.

48 MANW: AA IV $473=\mathrm{WW}$ V 18. 
Schema aber zur Vollständigkeit eines metaphysischen Systems, es sei der Natur überhaupt, oder der körperlichen Natur insbesondere, ist die Tafel der Kategorien"49.

Dieses Schema ermöglicht die Erstellung eines Klassifikationssystems, das im Sinne einer Topik "nicht leicht die Stelle verfehlen [läßt], dahin ein jeder Begriff eigentümlich gehört, und zugleich diejenige leicht bemerken, die noch leer ist." (A83 B109) Zum Verfahren der $M A N W$ führt Kant weiter aus:

\footnotetext{
"Unter die vier Klassen derselben [...] müssen sich auch alle Bestimmungen des allgemeinen Begriffs einer Materie überhaupt, mithin auch alles, was a priori von ihr gedacht, was in der mathematischen Konstruktion dargestellt, oder in der Erfahrung, als bestimmter Gegenstand derselben, gegeben werden mag, bringen lassen. ${ }^{50}$
}

Kant unterscheidet hier strikt zwischen den drei Disziplinen, die sich mit dem Begriff der Materie und dem, was durch ihn bezeichnet wird, auf ihre je eigene Weise beschäftigen: a priori denken (Metaphysik), in der Konstruktion darstellen (Mathematik), in der Erfahrung geben (Physik bzw. Naturwissenschaft). Die Vermittlung der Disziplinen durch die allgemeine Metaphysik bedeutet nicht, daß die $M A N W$ alle drei Erkenntnisweisen repräsentieren. In der Vorrede begründet Kant ja gerade die Notwendigkeit,

"die metaphysischen Anfangsgründe der Körperlehre nicht allein von der Physik, welche empirische Prinzipien braucht, sondern selbst die rationalen Prämissen derselben, die den Gebrauch der Mathematik in ihr betreffen, abzusondern [...] und sie als ein besonderes Ganze systematisch darzustellen." ${ }^{51}$

Die MANW sollen die Mathematisierbarkeit physikalisch bestimmbarer Objekte begründen, nicht selber ausführen. Welches Verfahren Kant dafür vorsieht, kann durch die Interpretation eines zentralen Textstückes der Vorrede präzisiert werden:

\footnotetext{
"Damit aber die Anwendung der Mathematik auf die Körperlehre, die durch sie allein Naturwissenschaft werden kann, möglich werde, so müssen Prinzipien der Konstruktion der Begriffe, welche zur Möglichkeit der Materie überhaupt gehören, vorangeschickt werden; mithin wird eine vollständige Zergliederung des Begriffs von einer Materie überhaupt zum Grunde gelegt werden müssen, welches ein Geschäfte der reinen Philosophie ist, die zu dieser Absicht sich keiner besonderen Erfahrungen, sondern nur dessen, was sie im abgesonderten (obzwar an sich empirischen) Begriffe selbst antrifft, in Beziehung auf die reinen Anschauungen im Raume und der Zeit (nach Gesetzen, welche schon dem Begriffe der Natur überhaupt wesentlich anhängen) bedient, mithin eine wirkliche Metaphysik der körperlichen Natur ist. ${ }^{52}$
}

In diesem vielzitierten Textstück bestimmt Kant Aufgabe, Verfahren und die wissenschaftssystematische Stellung der $M A N W$. Die Etablierung der Körperlehre als Naturwissenschaft gelinge durch den Nachweis, daß die für den Gegenstandsbereich der Körperlehre konstitutiven Begriffe mathematisch exakt beschrieben werden können. Dieser Nachweis setze eine philosophische Grundlegung im Sinne der "vollständigen Zergliederung" des Begriffs von einer Materie überhaupt voraus. Die Hinsichten, unter denen diese Zergliederung durchzuführen sei, weise das Verfahren als das einer "wirklichen Metaphysik der körperlichen Natur" aus. 
Nicht weniger unverständlich als der epistemische Status des "abgesonderten (obzwar an sich empirischen) Begriffs" der Materie ist den Interpreten das Verfahren der "Zergliederung des Begriffs von einer Materie überhaupt" geblieben. Man spricht hiervon in paradoxen Wendungen als einer "synthetischen Zergliederung"53, "metaphysischen Zergliederung"54, "empirischen Analyse" im Sinne des empirisch Sprachanalytischen ${ }^{55}$ oder als Analysis im Sinne der von Kant so genannten 'regressiven Lehrart', wonach Kant von Newtons Begriff der (trägen) Masse ausgehe, "als ob er gegeben sei" und "mit Hilfe der Kategorientafeln Schritt für Schritt die Bedingungen, unter denen er allein möglich ist", rekonstruiere. ${ }^{56}$

Das Ergebnis dieser Zergliederung wird kontrovers als Gewinnung reiner (Plaaß, Gloy), nicht-reiner (Cramer) oder empirischer (Hoppe) Begriffe bestimmt. Es ist wiederum dieses komplexe Verfahren der Zergliederung nach Maßgabe der Kategorientafel im Sinne der 'Durchführung' des Begriffs der Materie durch die Funktionen der Verstandesbegriffe, das das Verfahren der metaphysischen Konstruktion als 'Anwendung' transzendentaler Prinzipien beschreiben soll. Daß das Textstück in der Regel nur ausschnittsweise zitiert wird, mag an seiner auf den ersten Blick unübersichtlichen Verfassung liegen, verleitet jedoch zu einer Vernachlässigung des Kontextes, in dem Kant die fraglichen Terme einführt. Näher betrachtet, präzisiert Kant hier, wie eingangs paraphrasiert, das Programm der MANW nach Aufgabe, Verfahren und Status, und zwar in der Form einer klaren Gedankenführung, der ein ebenso klares, wenn auch komplexes Satzgefüge entspricht. Den Kern der Argumentation bilden zwei Hauptsätze, die jeweils das Gerüst der durch Semikolon getrennten Teile der Satzperiode ausmachen: "[...] so müssen Prinzipien der Konstruktion der Begriffe [...] vorangeschickt werden; mithin wird eine vollständige Zergliederung des Begriffs von einer Materie überhaupt zum Grunde gelegt werden müssen [...]."

Die Verknüpfung beider Hauptsätze durch "mithin" deutet auf die für die Argumentation explikative Funktion des zweiten Teils, der denn auch Merkmale eines entfaltenden Satzbaus trägt (Reihung, syntaktische Kontur durch adversative Konjunktionen, Klammern, Appositionen). Der zweite Hauptsatz endet mit einem wiederum durch "mithin" eingeleiteten Nachsatz, dessen weniger starke, aber deutliche Zäsur nur scheinbar auf einem Satzbruch beruht und den Relativsatz aufnimmt: "[...] welches ein Geschäfte der reinen Philosophie ist [...], mithin eine wirkliche Metaphysik der körperlichen Natur ist."

Wie der relativische Anschluß "welches" an den zweiten Hauptsatz zeigt, wird die dort geforderte 'Grundlegung', und zwar als ein 'Geschäft der reinen Philosophie' im Sinne der 'Zergliederung des Begriffs', gerade als das Verfahren der 'Metaphysik der körperlichen Natur' bestimmt. Beide Teilsätze spannen gleichsam den Rahmen auf, in den die Liste der Bedingungen eingetragen wird, unter denen 'diese Absicht' der rein philosophischen Grundlegung umzusetzen ist. Dabei steht in Frage die Beziehung zwischen dem, "was sie [die Philosophie] im abgesonderten (obzwar an sich empirischen) Begriffe selbst antrifft"

\footnotetext{
53 Plaaß (1965) 77.

54 Hoppe (1969) 57, Büchel (1987) 10.

55 Buchdahl (1984) 116f., 128.

56 Falkenburg (1987) 57.
} 
und dem, worauf es 'in Beziehung' gesetzt wird: "in Beziehung auf die reinen Anschauungen im Raume und der Zeit (nach Gesetzen, welche schon dem Begriffe der Natur überhaupt wesentlich anhängen)".

Die Erläuterung dieser Textpassage wirft besondere Schwierigkeiten auf, setzt sie doch schon ein Verständnis der kritizistischen These der Identifizierung von Natur- und Erfahrungsbegriff sowie des Beweiszieles der $M A N W$ selber voraus, daß die Erkenntnis bestimmter Naturdinge a priori möglich sei. ${ }^{57}$ Ich möchte zunächst nur zeigen, daß (nicht wie, s. Kap.5) im Sinne der genannten Voraussetzung der Nachweis von These und Beweisziel zu führen ist. Es läßt sich aufweisen, daß dasjenige, was hier 'in Beziehung' gesetzt wird, durch Kants Begriff der Natur vermittelbar ist. Dabei ergibt sich das, was vermittelt wird, aus der Differenz des Begriffs eines 'bestimmten Naturdinges' und des Begriffs einer 'Natur überhaupt'.

Ich gehe in zwei Schritten vor: (a) Anhand des Naturbegriffs expliziere ich, was hier vermittelbar und zugleich einer 'vollständigen Zergliederung' fähig sein soll. (b) Daß und wie diese Zergliederung im Kontext der 'rein philosophischen Grundlegung' aufzufassen ist, versuche ich an der Argumentationsstruktur der gesamten Satzperiode näher zu bestimmen. Diese Erörterungen sollen zeigen, daß weder das oben exponierte 'Anwendungsproblem' noch das 'Zergliederungsproblem' die Annahme eines Methodenbegriffs der 'metaphysischen Konstruktion' nahelegen oder gar zwingend machen. Die Begründungen und die Verwendung dieses Begriffs in der Forschung sollen dann in Kap.3 einer eingehenden Kritik unterzogen werden.

(a) Die von Kant geforderte 'vollständige Zergliederung' des Begriffs von einer Materie überhaupt bezieht sich, wie dargelegt, auf den Inhalt des 'abgesonderten (obzwar an sich empirischen) Begriffs der Materie', sofern eben dieser Inhalt 'in Beziehung auf die reinen Anschauungen im Raume und der Zeit' steht, und zwar 'nach Gesetzen, welche schon dem Begriffe der Natur überhaupt wesentlich anhängen'. Eine logische Analysis des Begriffs der Materie erfüllt die gestellten Bedingungen aus zwei Gründen nicht: Nach Kants Bestimmung der formalen Logik lassen sich die Merkmale des (empirischen) Begriffs nicht 'vollständig zergliedern' oder 'deutlich machen'.58 Zudem berücksichtigt die Logik gerade nicht das "Real - oder Natur-Wesen der Dinge", zu dessen Erkenntnis die Prädikate bestimmbar sein müßten, "von denen alles, was zu seinem Dasein gehört, als Bestimmungsgründen, abhängt." ${ }^{59}$ Nun gehört es zum Programm der $M A N W$, daß, sofern sie sich mit Naturdingen beschäftigt, sie gerade die "Prinzipien der Notwendigkeit dessen, was zum Dasein eines Dinges gehört"60 thematisiert. Dies ist aber "ein Geschäfte der reinen Philosophie" und fällt nicht in "das Geschäft der Logik"61. Kant nimmt den Begriff der Natur in zwei Bedeutungen, die nicht unabhängig voneinander explizierbar sind: Danach bezeichnet Natur (materiell) den 'Inbegriff' der Erscheinungen (Raum, Zeit und das, was

$57 M A N W$ : AA IV $470=\mathrm{WW}$ V 15 .

58 L: AA IX $63=$ WW III 491.

$59 \quad$ L: AA IX $61=$ WW III 489.

$60 M A N W$ : AA IV $469=\mathrm{WW}$ V 13.

61 L: AA IX $63=$ WW III 491. 
beide erfüllt - den Gegenstand der Empfindung); (formell) den 'Inbegriff' der Regeln, unter denen alle Erscheinungen stehen müssen, wenn sie Erfahrung sein sollen. ${ }^{62}$

"Denn wir kennen Natur nicht anders, als den Inbegriff der Erscheinungen, d.i. der Vorstellungen in uns, und können daher das Gesetz der Verknüpfung nirgend anders, als von den Grundsätzen der Verknüpfung derselben in uns, d.i. den Bedingungen der notwendigen Vereinigung in einem Bewußtsein, welche die Möglichkeit der Erfahrung ausmacht, hernehmen." 63

Im Hinblick auf die allgemeinen Naturgesetze bzw. transzendentalen Grundsätze des Verstandes sind "Natur und mögliche Erfahrung ganz und gar einerlei". ${ }^{4}$ Für unser Ausgangszitat heißt dies: Was 'in Beziehung' gesetzt wird, ist der Begriff der Materie, sofern er als Erscheinung in Raum und Zeit empirisch bestimmbar sein soll, und der Begriff einer Natur überhaupt, sofern dadurch die "Gesetzmäßigkeit der Erscheinungen in Raum und Zeit" (B165) bezeichnet wird. Das Resultat der Vermittlung beider Begriffe ist nun zugleich der Ausgangspunkt für die vollständige Zergliederung des Materiebegriffs, der dann als besonderes Naturding den Begriff der Natur als dasjenige 'erste innere' Prinzip $^{65}$ ausdrückt, das es erlaubt, die zum Dasein der Dinge gehörigen Bestimmungen zu erschließen, und zwar in 'immanentem', d.h. 'physischem' Vernunftgebrauch, der dabei auf Natur in materieller Bedeutung, den Bereich der Gegenstände unserer Sinne, begrenzt bleibt. ${ }^{66} \mathrm{Da}$ die Kategorien gerade "die Natur der Dinge"67 bezeichnen, ist der Begriff des besonderen Naturdinges anhand des kategorialen Schemas vollständig erschließbar.

(b) Zunächst gehe ich auf den Teil der Argumentation ein, als dessen Explikation dieser hier vorläufig dargestellte Sachverhalt der zweiten Satzperiode ('Grundlegung') fungiert. Wie die konjunktionskorrelative Verknüpfung anzeigt (damit ... so), formuliert der erste Hauptsatz selbst schon eine Anweisung, deren Zweck der die Textpassage einleitende Nebensatz so bestimmt: "Damit aber die Anwendung der Mathematik auf die Körperlehre, die durch sie allein Naturwissenschaft werden kann, möglich werde [...]".

In Frage steht die Möglichkeit der Anwendung der Mathematik auf den speziellen Gegenstandsbereich der Körperlehre. Zwar gilt, daß Naturwissenschaft "nur vermittelst der Mathematik"68 möglich ist, doch nur insofern, als sich darin Erkenntnis a priori befindet, also wie oben ausgeführt, metaphysische Erkenntnis vorgängig ist. Genaugenommen ist mit der Forderung der Mathematisierung der Körperlehre schon die Aufgabenstellung der $M A N W$ präzisiert, so daß Kant am Schluß der Passage emphatisch von einer "wirklichen" Metaphysik der körperlichen Natur spricht, wo er sonst noch allgemeiner von der "Möglichkeit einer mathematischen Naturlehre" redet. ${ }^{69}$

Die hier zunächst implizit geforderte 'Besonderung' des Gegenstandsbereichs ist maßgeblich abhängig von dem oben als 'Inhalt' des Begriffs der Materie Umschriebenen, das im Sinne philosophischer Grundlegung 'zergliedert' werden soll. Die Anweisung zur

62 $P$ \$6: AA IV $318=\mathrm{WW}$ III $186 \mathrm{f}$.

63 Ebd.: AA IV $319=$ WW III $187 f$.

64 Ebd.: AA IV $320=$ WW III 189.

$65 M A N W$ : AA IV $467=\mathrm{WW}$ V 11.

66 Vgl. Plaaß (1965) 25-31.

${ }^{67} M A N W$ : AA IV $474=\mathrm{WW}$ V 20.

$68 M A N W$ : AA IV $470=\mathrm{WW}$ V 15.

69 MANW: AA IV $473=\mathrm{WW}$ V 18. 
Umsetzung der gestellten Aufgabe lautet entsprechend: "[...] so müssen Prinzipien der Konstruktion der Begriffe, welche zur Möglichkeit der Materie überhaupt gehören, vorangeschickt werden $[\ldots] "$.

Wenn diese Anweisung im zweiten und explikativen Teil als besagte philosophische Grundlegung realisiert wird, bezieht sich die Rede vom Voranschicken solcher Prinzipien nicht etwa auf exponierte Stellen in den $M A N W$, sondern damit sind entsprechend der wissenschaftssystematischen Themenstellung des Vordersatzes (die Vermittlung von Mathematik und Naturwissenschaft durch Metaphysik) die MANW selber gemeint, sofern sie als 'besonderer Grundteil' der allgemeinen Physik fungieren. Die 'Prinzipien' der Konstruktion lassen sich somit als die 'metaphysischen Anfangsgründe' der Naturwissenschaft identifizieren. Entsprechend geht es Kant in den MANW nicht um die Anwendung der Mathematik, sondern um die Aufklärung der Voraussetzungen ihrer Anwendung. 'Konstruktion' läßt sich hier ohne Mühe als 'mathematische Konstruktion der Begriffe' im Sinne der spezifischen Erkenntnisart der Mathematik verstehen.

Die MANW liefern also nicht die mathematische Konstruktion selber, sondern deren Prinzipien, wobei es nicht um die Konstruktion von Begriffen überhaupt, sondern einer besonderen Klasse von Begriffen geht: nämlich solchen, "welche zur Möglichkeit der Materie überhaupt gehören". Als Beispiele für solche Begriffe führt Kant in der Vorrede an: Bewegung, Erfüllung des Raums, Trägheit. Der Gebrauch dieser 'physikalischen Leitbegriffe' (Plaaß) läßt sich erst durch ihr Auftreten in 'metaphysischen Prinzipien' rechtfertigen, die den Begriff der Materie dann "a priori zur Anwendung auf äußere Erfahrung tauglich machen." ${ }^{70}$ Im Unterschied zu den transzendentalen Prinzipien sind die $M A N W$ ein System von Sätzen a priori, die nicht auf die 'Natur überhaupt', sondern auf die 'besondere Natur' von Dingen gehen. Die $M A N W$ beschäftigen sich auch nicht mit 'Dingen überhaupt', sondern mit bestimmten Naturdingen, sofern deren Möglichkeit a priori erkannt werden soll..$^{71}$

Die Lösung der Aufgabe, die Möglichkeit bestimmter Naturdinge a priori zu erkennen, setzt voraus: daß ein Ding "außer dem Gedanken (als existierend) gegeben werden kann" und daß die Begriffe, die für die Bestimmbarkeit der Naturdinge konstitutiv sind, sich a priori in der reinen Anschauung geben lassen. ${ }^{72}$ Die Einlösung dieser Bedingungen kann nicht bedeuten, daß die Möglichkeit des Naturdinges a priori aus dem bloßen Begriff von ihm analytisch hergeleitet wird, sondern schließt den Rekurs auf empirische Daten ein. Es ist auch nicht gefordert, daß die $M A N W$ das Dasein der Dinge in der reinen Anschauung a priori darstellen sollen. Vielmehr treten in den Urteilen der MANW Begriffe auf, die für den Dingcharakter von etwas, dem Dasein zukommt, konstitutiv sind ${ }^{73}$, aber so, daß über diese Dinge Aussagen von mathematischer Exaktheit möglich sind. Diese Begriffe stehen zwar im Kontext der "Prinzipien der Notwendigkeit dessen, was zum Dasein eines Dinges 
gehört"74, sind aber kraft ihrer konstitutiven Funktion für materielle Entitäten mathematisierbar.

$74 M A N W$ : AA IV $469=\mathrm{WW}$ V 13 


\section{Der Begriff der metaphysischen Konstruktion}

Kants Kritik an der von Newton dominierten Naturwissenschaft seiner Zeit richtet sich gegen die "Vermengung"1 metaphysischer und mathematischer Prinzipien, die, wie er schreibt, "unbewußt" allen Naturphilosophen unterlaufe, "welche in ihrem Geschäft mathematisch verfahren wollten"2. Seine Forderung nach der Entflechtung dieser "ungleichartigen Prinzipien"3 will er mit den $M A N W$ durch die Etablierung eines eigenen metaphysischen Systemteils einlösen, der dem mathematischen Teil der theoretischen Physik vorgeschaltet werden soll. Damit soll die Anwendung der Mathematik auf den Gegenstandsbereich der empirischen Physik allererst legitimiert werden nach Maßgabe der Prinzipien der transzendentalen Theorie der Erfahrung.

Seit den sechziger Jahren hat sich in der Forschungsliteratur die Meinung durchgesetzt, daß Kant die eigene Art von Schwierigkeiten, die sich aus dem Programm metaphysischer Anfangsgründe der empirischen Wissenschaft von der Natur (Physik) ergeben, nur durch die Einführung des an der mathematischen Konstruktion orientierten Verfahrens der metaphysischen Konstruktion zu lösen vermochte. Es ist klar, daß Kant mit der Bildung dieses mutmaßlichen Methodenbegriffs 1786 die von ihm in der $K r V$ entwickelte prinzipielle Differenz philosophischer und mathematischer Erkenntnisart unterliefe. Damit sähe er sich zugleich dem Vorwurf ausgesetzt, er habe gerade gegen "die Definition der Philosophie [...], welche der Verfasser der Kritik d. r. V. für sein eigenes, nicht unerhebliches, Produkt ausgibt" - so reklamiert Kant $1797^{4}$ - verstoßen.

Ich werde die These, daß Kant in den $M A N W$ ein spezifisches Verfahren unter dem Namen 'metaphysische Konstruktion' einzuführen im Sinn hatte, einer Kritik ihrer Voraussetzungen unterziehen: In einer textkritischen Untersuchung (3.1.) sollen die Konstitution der Textstelle, an der der Begriff auftritt, bewertet und anhand der Analyse des Kontextes dieser Stelle alternative Konjekturen diskutiert werden. In 3.2. gebe ich einen Überblick über die Vorschläge zur Interpretation des Begriffs. In 3.3. gehe ich den Voraussetzungen nach, auf denen Plaaß' Etablierung des Verfahrens der metaphysischen Konstruktion beruht.

\subsection{Textkritik}

Die einzige Textstelle in den $M A N W$ (und im gesamten Kantischen Euvre), die als Beleg für den mutmaßlichen Methodenbegriff angegeben wird, lautet:

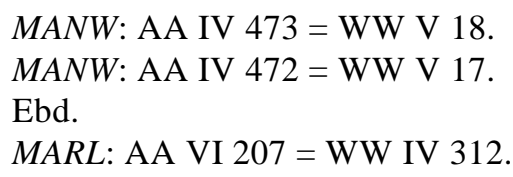


"Um deswillen habe ich für nötig gehalten, von dem reinen Teile der Naturwissenschaft (physica generalis), wo metaphysische und mathematische Konstruktionen durch einander zu laufen pflegen, die erstere, und mit ihnen zugleich die Prinzipien der Konstruktion dieser Begriffe, also der Möglichkeit einer mathematischen Naturlehre selbst, in einem System darzustellen." ${ }^{5}$

An der umstrittenen Textstelle spricht Kant nicht eindeutig von 'metaphysischer Konstruktion', sondern von "metaphysischen und mathematischen Konstruktionen". Der elliptisch formulierte Ausdruck hat denn auch Kritiker dieses neuen Konstruktionstyps zu der Vermutung veranlaßt, das Attribut "metaphysische" sei nicht auf "Konstruktionen", sondern auf ein Wort zu beziehen, das 'ausgefallen' und sinngemäß durch "Prinzipien" oder "Begriffe" ergänzt werden könne. ${ }^{6}$ Die Textstelle ist korrupt, zumindest weist sie drei grammatische Irregularitäten auf:

(a) Wenn sich das Akkusativobjekt "die erstere" des satzwertigen Infinitivs "darzustellen" auf den Ausdruck "metaphysische [...] Konstruktionen" bezöge, dann müßte entweder die Flexionsform korrigiert ("die erstere $n^{17}$ ) oder der Artikel "die" getilgt werden. Der Ausdruck "mit ihnen" nähme dann eindeutig "metaphysische [...] Konstruktionen" auf. Fraglich bleibt dann allerdings, welcher Konstruktionstyp in der Wendung "die Konstruktion dieser Begriffe" angesprochen ist, wenn "mit ihnen", d.h. mit den metaphysischen Konstruktionen, "zugleich die Prinzipien der Konstruktion dieser Begriffe" darzustellen sind.

(b) Will man die Flexionsform "die erstere" nicht auf nachlässigen oder veralteten Sprachgebrauch zurückführen, so kann sie auch als Akkusativobjekt Singular gelesen werden, in dem Sinn, daß metaphysische und mathematische Konstruktionstypen jeweils einzeln betrachtet werden. Dieser Version korrespondierte der Singular im Ausdruck "Konstruktion dieser Begriffe"; das Pronomen "mit ihnen" müßte sich dann jedoch wiederum auf beide Konstruktionstypen beziehen. Hier wäre zu entscheiden, ob man zwischen "die erstere" und "und mit ihnen zugleich" durch ein ungrammatisches Komma eine Zäsur setzt (so die Originalausgabe 1786) oder nicht (in AA IV 473 ist das Komma getilgt).

Hält man wie in den Varianten (a) und (b) an der Lesart "metaphysische Konstruktion" fest, so sind der Gebrauch und Bezug des Demonstrativpronomens im Ausdruck "dieser Begriffe" nicht erklärbar. Von den fraglichen Begriffen spricht Kant weder in dem vorliegenden noch in den beiden, zudem durch einen Absatz getrennten vorangehenden Sätzen. Erst dann ist eine Textstelle vorangestellt, zu der sich ein Bezug ergibt; allerdings spricht Kant hier gerade von den "metaphysischen Prinzipien", die den Begriff der Materie "a priori zur Anwendung auf äußere Erfahrung tauglich machen, als des Begriffs der Bewegung, der Erfüllung des Raums, der Trägheit, u.s.w."8 Sollte Kant auf eben diese Begriffe verweisen, so spricht dies für die von Hoppe favorisierte Konjektur, zumal Kant

\footnotetext{
MANW: AA IV $473=\mathrm{WW}$ V 18.

Hoppe (1969) 57, Gloy (1976) 8.

Mit dieser unausgewiesenen Konjektur zitiert Gloy (1976) 8 die Textstelle; dazu schon Büchel (1987)

13f. Anm.16.

$8 \quad M A N W$ : AA IV $472=\mathrm{WW}$ V 17.
} 
zufolge die $M A N W$ eben jene "Prinzipien der Konstruktion der Begriffe, die zur Möglichkeit der Materie überhaupt gehören", formulieren. ${ }^{9}$

(c) Daß die aufgeführten Irregularitäten nicht befriedigend aufgeklärt werden können, geht auf einen Satzbruch zurück. Das Gerüst des Satzes lautet:

"[...] habe ich für nötig gehalten, von dem reinen Teile der Naturwissenschaft [...], wo metaphysische und mathematische Konstruktionen durch einander zu laufen pflegen, die erstere [...] in einem System darzustellen."

Kant hat offensichtlich den Gedankengang, den der Objektsatz einleitet, nicht abgeschlossen. So muß die Wendung "von dem reinen Teile" dem Sinn nach anders fortgeführt werden als durch das Verb "darzustellen"; man erwartet vielmehr den Abschluß des Gedankens durch 'abzusondern', das dann nach "die erstere" einzufügen ist. Diesen hier ergänzten Gedanken greift Kant im folgenden Satz unmittelbar auf: "Diese Absonderung [...]". Und der vorangehende Satz hebt gerade die "Wichtigkeit" hervor, "um deswillen" Kant diese Absonderung vornehmen will: Es geht darum, "zum Vorteil der Wissenschaften ungleichartige Prinzipien von einander zu scheiden, jede in ein besonderes System zu bringen". ${ }^{10}$

Der Befund der Textkritik zeigt, daß die Verwendung des Ausdrucks 'metaphysische Konstruktion' nicht eindeutig belegbar und darüber hinaus die Konstitution der Textstelle bis hin zum Satz- und Gedankenbruch fehlerhaft ist. Zudem spricht die Kontextanalyse eher für die von den Kritikern angebotene Konjektur. Eine textkritische Untersuchung kann Zweifel wecken und erhärten, eine Beweisführung für oder gegen die fragliche These leistet sie, zumal unter der Annahme von Konjekturen, nicht. Sie kann aber durch eine inhaltlich orientierte Kritik ergänzt werden.

\subsection{Interpretationen (1965-1990)}

Der Konstruktionsbegriff in den MANW scheint von jeher das besondere Interesse der Interpreten auf sich gezogen zu haben; er hat bei den Interpreten Anstoß erregt oder ihre Phantasie beflügelt. Das gilt gleichermaßen für die frühe Kant-Rezeption und die Adepten der Schellingschen Naturphilosophie wie für die zeitgenössische Forschung. Soweit ich sehe, wird der Begriff der metaphysischen Konstruktion zur Bezeichnung des spezifischen Verfahrens der MANW erstmals von Schäfer (1962/1966) und Plaaß (1965), vermutlich unabhängig voneinander, verwendet. Plaaß' Interpretationsvorschlag hat sich als der wirkmächtigere in der Forschung durchgesetzt, so daß es trotz einzelner Vorbehalte wegen der unsicheren Textbasis als ausgemacht gilt, mit Verweis auf Plaaß von einem Verfahren der metaphysischen Konstruktion zu sprechen. Gleichwohl weichen nicht nur die Deutungen dieses Verfahrens bei Schäfer und Plaaß voneinander $a b$, sondern auch die Darstellungen, die sich direkt auf Plaaß beziehen oder Plaaß' Interpretation auch nur wiedergeben wollen. So hat man dieses Verfahren auch durch 'Arbeitstitel' wie 
"konstruktive Konstruktion"11 oder "naturwissenschaftliche Konstruktion"12 umschrieben. Es ist daher angebracht, Plaaß' Explikation des Verfahrens näher zu untersuchen (3.3.). Hier beschränke ich mich auf die Wiedergabe der Formel, die Plaaß seiner Deutung gegeben hat: 'Analog' zur Bestimmung mathematischer Konstruktion als der "Erzeugung reiner Anschauungen" nennt er die metaphysische Konstruktion die "Erzeugung reiner Begriffe". ${ }^{13}$

Über die Vorschläge zur Interpretation des Konstruktionsbegriffs in den MANW sei zunächst ein kurzer Überblick gegeben. Es ist klar, daß diese wenigen Zitate den einzelnen Interpretationsansätzen nicht gerecht werden. Sie sollen verdeutlichen, mit welchen Konnotationen der Konstruktionsbegriff und das Problem, für das er einstehen soll, behaftet sind. Damit ist freilich auf ein noch gewichtigeres Problem vorausgewiesen: nicht nur zu bestimmen, was der Kantische Konstruktionsbegriff besagt, sondern eben auch, was die Differenz von philosophischer und mathematischer Erkenntnisart besagt, die durch den Konstruktionsbegriff bezeichnet wird.

Schon Adickes spricht von einer "Umbiegung", "Abschwächung" und "Einschränkung" gegenüber dem in der $\mathrm{KrV}$ bestimmten Konstruktionsbegriff, der in den MANW nurmehr der "Veranschaulichung" diene. "Bei der Materie vor allem handelt es sich gar nicht um bloße Formen und Gestalten, sondern um Qualitäten, um einen Inhalt, eben - um die Materie selbst." Daher könne von einer "etwaigen Konstruktion des Begriffs der Materie und selbst des der Bewegung keine Rede sein." ${ }^{14}$

In ähnlicher Weise tritt nach Ende Konstruktion in den MANW nur in einer "Nebenbedeutung" der mathematischen Konstruktion auf; Konstruktion sei "nicht mehr Beweismittel, sondern nur noch Mittel zur Veranschaulichung von Begriffen". ${ }^{15}$ Im Unterschied $\mathrm{zu}$ Adickes stellt sie jedoch fest: "Der ursprüngliche schematische Konstruktionsbegriff ist in den 'Metaphysischen Anfangsgründen der Naturwissenschaft' abgeschwächt (obgleich die Definition der Konstruktion mit der in der 'Kritik' gegebenen übereinstimmt), da er nicht nur auf Größen, sondern auch auf Qualitäten angewendet wird." 16

Schäfer geht davon aus, "daß der Begriff der Konstruktion tatsächlich in einer neuen Bedeutung zu nehmen ist". ${ }^{17}$ "Es wird der mathematischen Konstruktion ausdrücklich eine metaphysische zur Seite oder voran gestellt." ${ }^{18}$ Schäfer sieht 'Konstruktion als Methode der Metaphysik der Natur', die keine "Mischform von transzendentaler und empirischer Methode" sei $^{19}$, sondern "einen eigenen Ansatz" ${ }^{20}$ anzeige. "Die Methode nun, nach der Kant den Bezug der Begriffe auf die Erfahrung herzustellen sucht, nennt er [...]

Ende (1973) 28.

Büchel (1987) 14.

Plaaß (1965) 76.

Adickes (1924) 268.

Ende (1973) 30.

Ebd. $28 f$.

Schäfer (1966) 32 Anm. 45.

Ebd. 32.

19 Ebd. 30.

20 Ebd. 38. 
metaphysische Konstruktion." ${ }^{21}$ Dazu führt Schäfer aus: "Das Mannigfaltige, das in der metaphysischen Konstruktion geeint werden soll, besteht in den durch die Zergliederung des Begriffs der Materie gewonnenen Grundbestimmungen".22

"Entsprechend" der mathematischen Konstruktion, so Stuhlmann-Laeisz, werde in der metaphysischen "die bloße, unbestimmte Form des Denkens von Gegenständen überhaupt gemäß dem Inhalt eines vorgegebenen Begriffs [...] bestimmt, und hierdurch werden diejenigen diskursiven Vorstellungen 'konstruiert', durch die der im Begriff bedeutete Gegenstand als ein solcher, d.h. gemäß der durch die Kategorien vorgegebenen Form von gegenständlichem Denken überhaupt, gedacht werden kann."23

Buchdahl weist auf die Absicht Kants hin, "die zwei Seiten der 'metaphysischen' und 'mathematischen' Konstruktion scharf auseinander zu halten". Am Beispiel der MAPhor führt er aus: "Kants Vorgehen besteht hier darin, Bewegung [...] als eine 'Veränderung der äußeren Verhältnisse (eines Dinges) zu einem gegebenen Raum' auszudrücken ([AA IV]482), um auf diese Art der 'metaphysischen Konstruktion' ([AA IV]473) Einheitlichkeit mit der 'mathematischen', d.h. geometrischen, Konstruktion zu erzielen." 24

Falkenburg spricht von dem "Verfahren der 'metaphysischen Konstruktion'" als von dem apriorisch-synthetischen Verfahren, "mit dem er [Kant] den vollständigen Begriff der Materie ausgehend von dem der Bewegung als dem Bestimmbaren an ihr entwickelt". ${ }^{25}$ "Kant legt in der Vorrede der MAG die Bewegung als das Bestimmbare der Materie fest und vollzieht dann in vier Hauptstücken die eigentliche 'metaphysische Konstruktion', nämlich die Bestimmung des Materiebegriffs, indem er den Begriff der Bewegung unter die vier Kategorien bringt bzw. diese durch jenen spezifiziert." ${ }^{26}$ Der Bewegungsbegriff sei "der einzige für die 'metaphysische Konstruktion' taugliche Ausgangspunkt, weil nur er eines (hier metaphysischen) Schematismus fähig ist". ${ }^{27}$

Friedman nimmt den Begriff der metaphysischen Konstruktion im Rekurs auf Plaaß sogar für die MAPhän in Anspruch, obwohl der Begriff dort gar nicht auftritt. "The Phaenomenology of MAN depicts a constructive procedure for 'transforming appearance (Erscheinung) into experience (Erfahrung)' [...] This procedure therefore seeks to construct an objective spatio-temporal framework". ${ }^{28}$ - "How does the construction now proceed? First of all, the abstract principles of the first Critique - in particular, the Analogies of Experience - are to be further specified by means of Kant's 'metaphysical construction' of the concept of matter in $M A N$, which renders it 'a priori suitable for application to outer experience'." 29

29 Ebd. 54. 
Carrier: "Kants Frage ist also, wie Materie (real) möglich sei, und die Antwort darauf wird durch das Verfahren der metaphysischen Konstruktion gegeben, welches durch Anwendung der Kategorientafel auf einen besonderen Begriff jene besonderen Bestimmungen erzeugen soll, die dem dadurch bezeichneten Objekt zukommen müssen, soll es Gegenstand der Erfahrung werden." ${ }^{30}$

Waidhas bestimmt den Begriff der metaphysischen Konstruktion im Vergleich zur mathematischen als den umfassenderen Synthesisbegriff, der die "Einigung des Mannigfaltigen der Anschauung zu Bild und Begriff als Handlung des Subjekts" bezeichne. $^{31}$

Gloy hat ihre zunächst ${ }^{32}$ geäußerten Vorbehalte gegen den Begriff der metaphysischen Konstruktion offensichtlich verworfen und erläutert nunmehr diesen Begriff als "Darstellung der Anschauung in bezug auf Begriffe." ${ }^{33}$

Tuschling geht zwar davon aus, daß die $M A N W$ "das Durcheinanderlaufen metaphysischer und mathematischer Konstruktionen verhüten" sollten. ${ }^{34}$ Doch glaubt er zeigen zu können, "daß die MA sowohl metaphysische wie mathematische Erkenntnis sein wollen, sich also selbst widersprechen, etwas Unmögliches, ein Unding sind." ${ }^{35}$

Diese doch überraschende Vielzahl divergierender Deutungen läßt in der Forschung folgende Tendenzen erkennen:

- Es gilt als ausgemacht, daß Kant den Begriff der Konstruktion eindeutig der Kennzeichnung der mathematischen Erkenntnisart vorbehält. Ferner soll gelten, daß die Definition mathematischer Konstruktion in der $K r V$ und den $M A N W$ übereinstimmt. Dennoch wird behauptet, daß der Konstruktionsbegriff in den $M A N W$ verschiedene Modifikationen aufweist.

- Die Mehrzahl der Interpreten geht davon aus, daß der Konstruktionsbegriff in den MANW nicht oder nicht nur eine Abschwächung (Adickes, Ende) erfahre und in einer Nebenbedeutung (Ende) zu nehmen sei; vielmehr wird das Auftreten zweier Konstruktionstypen beobachtet, deren Verhältnis dann jedoch ganz unterschiedlich bestimmt wird.

- So wird die metaphysische Konstruktion der mathematischen bei- oder vorgeordnet (Schäfer); die metaphysische Konstruktion repräsentiere den umfassenderen Synthesisbegriff (Waidhas).

- Die Funktion der metaphysischen Konstruktion wird analog (Plaaß), entsprechend (Stuhlmann-Laeisz) oder parallel (Stegmaier) zur mathematischen Konstruktion bestimmt, nämlich als Erzeugung reiner Begriffe (Plaaß) oder als Konstruktion diskursiver Vorstellungen (Stuhlmann-Laeisz).

\footnotetext{
Carrier (1990) 170.

Waidhas (1985) 86.

Gloy (1976) 8-13.

Gloy (1990) 91.

Tuschling (1971) 116 Anm. 11.

Ebd. 115
} 
- Die Verdopplung des Konstruktionsbegriffs in den MANW macht die metaphysische Konstruktion zu einem Verfahren, dessen Anwendung die Einheitlichkeit mit der mathematischen Konstruktion (Buchdahl) zum Ziel hat.

- Aufzulisten bleibt die Vielzahl der Funktionen, die der metaphysischen Konstruktion im einzelnen zugeschrieben werden: Es sei ein Verfahren der Entwicklung des vollständigen Begriffs der Materie (Falkenburg), es leiste die Einigung von Mannigfaltigem (Schäfer, Waidhas), es stelle den Bezug von Begriff und Erfahrung her (Schäfer), es diene dem Nachweis der realen Möglichkeit der Materie (Carrier), es sei Darstellung der Anschauung (Gloy).

- Schließlich soll es Kant selbst gewesen sein, der die von ihm verfochtene Trennung beider Konstruktionsbegriffe unterlaufen habe (Tuschling).

\subsection{Plaaß' "Vermutung"}

Trotz auffälliger Differenzen in der Deutung des Begriffs der metaphysischen Konstruktion scheint doch die Etablierung dieses nach Plaaß der mathematischen Konstruktion 'analogen' - oder, wie es gelegentlich heißt, 'parallelen' - Verfahrens hinreichend attraktiv und plausibel zu sein. Die Voraussetzungen und Rechtfertigung der von Plaaß behaupteten 'Analogie' werden in der Regel unhinterfragt übernommen. Tatsächlich kommt Plaaß zu seiner These nur aufgrund einer 'analogen Vermutung ${ }^{136}$, am Beispiel der 'mechanischen Konstruktion' gibt er nurmehr eine 'metaphorische Beschreibung' des Verfahrens der metaphysischen Konstruktion. ${ }^{37}$

Plaaß zieht bei der Formulierung des Begriffs das mathematische Konstruktionsverfahren allenfalls als Vergleich zu heuristischen Zwecken heran, aus diesem Vergleich leitet er jedoch recht weitgehende Schlüsse ab. Die zentrale Textstelle lautet:

"Mathematische Konstruktion eines Begriffs ist die Erzeugung einer reinen Anschauung, die dem Begriff gemäß ist, d.h.: gemäß dem, was im Begriff inhaltlich enthalten ist, wird etwas a priori dargestellt in dem, was als Bedingung der Anschauung von Gegenständen gegeben ist.

Analog vermuten wir: metaphysische Konstruktion eines Begriffs heißt: gemäß dem inhaltlich im Begriff Enthaltenen etwas a priori darstellen in dem, was als Bedingung des Denkens gegeben ist, also in dem System der reinen Verstandesbegriffe; d.h. also: nicht Anschauungen, sondern Begriffe erzeugen, die, so wie die in der mathematischen Konstruktion erzeugte Anschauung angibt, wie in der Anschauung überhaupt dieser (im Begriff gedachte) Gegenstand angeschaut werden muß, angeben, wie dieser besondere 'Gegenstand ... bloß nach den allgemeinen Gesetzen des Denkens ... vorgestellt werden muß' (S XIV-XV), nämlich nach den Gesetzen des Denkens eines Gegenstandes, der Dasein soll haben können (bzw. als daseiend soll bestimmt werden können)." ${ }^{38}$

Ich beschränke mich hier auf die Erörterung der Voraussetzungen, auf die sich Plaaß bei der Herleitung seines Methodenbegriffs stützt: die Unterscheidung mathematischer und

36 Plaaß (1965) 74.

37 Ebd. 76: "[...] diesen besonderen Begriff [der Masse] unter der Kategorie der Substanz zu erzeugen, heißt die metaphysische Konstruktion des empirischen Begriffs der Materie, sozusagen in Ansehung derjenigen Dimension des 'Denkraumes', die durch die Substanzkategorie bezeichnet wird, durchzuführen."

38 Plaaß (1965) 74 bezieht sich auf MANW: AA IV $473=\mathrm{W}$ V 18. 
philosophischer Erkenntnisart und die Bestimmung mathematischer Erkenntnis als die Konstruktion der Begriffe. Plaaß legt es darauf an, Merkmale der mathematischen Erkenntnisart auf die philosophische zu übertragen. Dieses Konzept beruht offenbar auf zwei Voraussetzungen: Plaaß geht von der Disjunktion beider Erkenntnisarten aus, wobei philosophische Erkenntnis (diskursiv) auf die Analyse von Begriffen, die mathematische Erkenntnis (intuitiv) auf die 'Erzeugung reiner Anschauungen' beschränkt bleibe. ${ }^{39}$ Plaaß sieht das Spezifikum mathematischer Erkenntnis in ihren epistemischen und genetischen Momenten, nämlich in der 'Reinheit' und 'Erzeugung' der Anschauungen, so daß sich als Resultat der Übertragung auf philosophische Erkenntnis mit dem Begriff der metaphysischen Konstruktion ein Verfahren der "Erzeugung reiner Begriffe" ergebe.

Plaaß identifiziert die so erzeugten Begriffe mit denjenigen, die in den $M A N W$ durch die 'Anwendung' der transzendentalen Prinzipien des Begriffs einer Natur überhaupt auf den Begriff der Materie gewonnen werden und dort als die physikalischen Leitbegriffe (Bewegung, Undurchdringlichkeit, Trägheit) fungieren. Abgesehen davon, daß diese Begriffe selber gar nicht den epistemischen Status 'reiner' Begriffe aufweisen (2.2.), dient Plaaß' Etablierung des Verfahrens der metaphysischen Konstruktion einem Zweck, den Kants transzendentalphilosophische Begründung der Newtonschen Mechanik ohnehin schon erfüllen soll: nämlich die Quantifizierbarkeit derjenigen empirischen Sachverhalte auszuweisen, die durch eben jene Leitbegriffe bezeichnet und bestimmbar werden. Nach diesen Vorüberlegungen erwiese sich die Einführung des Begriffs der metaphysischen Konstruktion dann als überflüssig, wenn es gelingt, 'Konstruktion' in den $M A N W$ als einen Begriff zur Bezeichnung des mathematischen Verfahrens (freilich in der systematischen Perspektive der transzendentalen Theorie der Erfahrung) auszuweisen. ${ }^{40}$ Ein Grund dafür, daß dieser Begriff dennoch als legitimierungsfähig gilt, liegt offenbar in der unzulänglichen Bestimmung der Differenz von mathematischer und philosophischer Erkenntnisart, die Kant durch den Ausdruck 'Konstruktion der Begriffe' bezeichnet. Wenn dies zutrifft, muß die Unterscheidung beider Erkenntnisarten anders als gemeinhin angenommen (und von Kant auch nahegelegt) interpretiert werden.

Plaaß' griffige Formel der 'Erzeugung reiner Anschauungen' reicht zur Kennzeichnung der mathematischen Erkenntnisart nicht aus. Abgesehen von dem ambivalenten Begriff der Erzeugung ist es gerade der Rekurs auf so etwas wie 'reine Anschauung', der die Interpreten bislang Kants Konzept der Mathematik hat recht rätselhaft erscheinen lassen. Nun unternimmt Plaaß auch nicht den Versuch, den Konstruktionsbegriff so weit zu entwickeln, daß er in seiner Anwendung in Geometrie, Arithmetik oder gar Algebra verstehbar würde. Vielmehr scheint er unter der Hand auf einen Konsens im Verständnis wesentlicher Momente der Kantischen Interpretation mathematischer Urteile Synthetizität, Intuitivität, Apodiktizität - zu vertrauen.

39 Ebd. 70f.: Transzendentalphilosophie sei "für sich selbst unabhängig von der Anschauung und insofern Metaphysik aus bloßen Begriffen [...] und also im engeren Sinne keine Erkenntnis." - 81: "Mathematik erzeugt keine Begriffe, sondern kann nur Begriffe konstruieren, die ihr gegeben sind." - 62: Außerdem "kann der Begriff genaugenommen nur dann konstruiert werden, wenn er nichts anderes als 'eine reine Anschauung in sich' enthält, d.h. genauer: eine Synthesis der reinen Anschauung."

40 Vgl. Kap.5.4. 


\section{Konstruktion der Begriffe (I): "Darstellung des Begriffs in der Anschauung a priori"}

Die Diskussion um den Zwitterbegriff der metaphysischen Konstruktion weist darauf hin, daß die methodologischen Grundlagen der Differenz von mathematischer und philosophischer Erkenntnisart noch ungeklärt sind. Es ist die Frage zu stellen, worin das Kriterium der Differenz beider Erkenntnisarten besteht und wie es sich innerhalb der Theorie, die es formuliert, begründen läßt. In 4.1. werden die Merkmale eingeführt, mit denen Kant die mathematische Erkenntnisart als intuitiv, synthetisch und apodiktisch charakterisiert. In 4.2. diskutiere ich die These Hintikkas, daß und inwiefern Kant sich bei der Bestimmung der mathematischen Erkenntnisart am geometrischen Beweisverfahren Euklids orientiert. Daran anschließend, versuche ich in 4.3. (und weiter in Kap.7 und 9.1.) den Vorschlag auszuführen, Kants Konstruktionstheorem der Terminologie und der Sache nach von einem der transzendentalen Theorie der Erfahrung immanenten Standpunkt aus zu entwickeln.

\subsection{Zu Kants Bestimmung der mathematischen Erkenntnis als 'Konstruktion der Begriffe'}

Kants These, daß mathematische Urteile "insgesamt synthetisch" (B14) sind, hat wenig Zustimmung gefunden. Und angesichts der Forschungslage ist es auch nicht übertrieben, zu behaupten, daß die Charakterisierung der Synthetizität durch 'Konstruktion der Begriffe in der Anschauung', genauer in der reinen Zeitanschauung, bis heute nicht hinreichend aufgeklärt ist. Gegen Leibniz vertrat Kant jedenfalls die Auffassung, daß mathematische Urteile nicht in den Gesetzen der Logik enthalten oder daraus herleitbar sind. Allerdings war er nicht der Meinung, daß der Satz des Widerspruchs, den Leibniz als "le grand fondement des Mathematiques"1 ansah, im mathematischen Beweis nicht anwendbar oder ohne Geltung sei. Die Apodiktizität mathematischer Deduktionen folge gerade daraus, "daß die Schlüsse der Mathematiker alle nach dem Satze des Widerspruchs fortgehen" (B14). Daraus zu folgern, "daß auch die Grundsätze aus dem Satze des Widerspruchs erkannt würden" (B14), hält Kant jedoch für einen Irrtum. Die Geltung des Widerspruchsprinzips ist nach Kant also nur auf Folgerungsbeziehungen beschränkt. Kant gibt folgende Erläuterung:

"denn ein synthetischer Satz kann allerdings nach dem Satze des Widerspruchs eingesehen werden, aber nur so, daß ein anderer synthetischer Satz vorausgesetzt wird, aus dem er gefolgert werden kann, niemals aber an sich selbst." (B14) 
Nun behauptet Kant mit dieser Einschränkung zunächst nur, daß der Satz des Widerspruchs eine notwendige Bedingung für die Geltung synthetischer Sätze ist; die Synthetizität der Sätze wird dabei jedoch schlicht vorausgesetzt und bedarf einer eigenen Rechtfertigung. Kant räumt ein, daß "einige wenige Grundsätze, welche die Geometer voraussetzen", "wirklich analytisch" sind und daher auf dem Widerspruchsprinzip beruhen, aber nur, wie er sagt, "zur Kette der Methode und nicht als Prinzipien" (B16f.) dienen. Als Beispiele führt er das erste und achte Axiom aus Buch I der Euklidischen Elemente an. ${ }^{2}$ Diese Axiome finden tatsächlich nur in dem Teil des geometrischen Beweisverfahrens Anwendung, in dem (als apodeixis) analytisch gefolgert wird. ${ }^{3}$ Prinzipien sind sie insofern nicht, als nicht aus ihnen, sondern nach ihnen gefolgert wird. Doch ist der Gebrauch dieser Axiome im mathematischen Beweisverfahren dadurch gerechtfertigt, daß sie sich eben darauf beziehen, worauf die Synthetizität mathematischer Urteile ausschließlich beruht: Anschauung. ${ }^{4}$

"Anschauung muß also hier zu Hilfe genommen werden, vermittels deren allein die Synthesis möglich ist." (B16) Mithin ist der Gebrauch dieser analytischen Grundsätze zulässig, "weil sie in der Anschauung können dargestellt werden." (B17) Kant bezeichnet dieses Verfahren der Darstellung von Begriffen in der Anschauung näher als 'Konstruktion der Begriffe'. Zugleich markiert das Verfahren der Konstruktion die prinzipielle Differenz von mathematischer Erkenntnisart, die intuitiv ist, und philosophischer Erkenntnisart, die diskursiv ist.

"Die philosophische Erkenntnis ist die Vernunfterkenntnis aus Begriffen, die mathematische aus der

Konstruktion der Begriffe. Einen Begriff aber konstruieren heißt: die ihm korrespondierende Anschauung a priori darstellen." (A713 B741)

Daß auch analytische Grundsätze im mathematischen Beweis angewendet werden, weil sie in der Anschauung darstellbar sind, deutet schon darauf hin, daß es nicht die durch die Begriffe bezeichneten Sachverhalte sind, die die Differenz der Erkenntnisarten ausmachen, sondern das 'Konstruktion' genannte Verfahren des Gebrauchs von Begriffen. Nun soll Konstruktion durch die Bezugnahme auf Anschauung (in der Darstellung a priori) für die Synthetizität mathematischer Urteile einstehen. Kant behauptet aber nicht, daß philosophische Erkenntnis als Vernunfterkenntnis 'aus Begriffen' weder synthetisch sei noch sich auf Anschauung beziehe. ${ }^{5}$ Es ist vielmehr die mit der mathematischen Konstruktion verbundene Apriorität der Darstellung (der Begriffe in der Anschauung), die die eigentümliche Differenz der Erkenntnisarten bedingt.

Sieht man von den Intuitionisten $a b$, denen eine gewisse Nähe zu Kants Mathematikverständnis nachgesagt wird, so gilt seine Theorie spätestens seit der radikalen

2 Euklid (1980) 3: "Was demselben gleich ist, ist auch einander gleich." (1. Axiom) - "Das Ganze ist größer als der Teil." (8. Axiom)

Vgl. 4.2.

Vgl. A300 B356: "Der Ausdruck eines Prinzips ist zweideutig [...]. Die mathematischen Axiome (z.B. zwischen zwei Punkten kann nur eine gerade Linie sein,) sind sogar allgemeine Erkenntnisse a priori, und werden daher mit Recht, relativisch auf alle Fälle, die unter ihnen subsumiert werden können, Prinzipien genannt. Aber ich kann darum doch nicht sagen, daß ich diese Eigenschaft der geraden Linien überhaupt und an sich, aus Prinzipien erkenne, sondern nur in der reinen Anschauung."

5 Vgl. A720f. B748f. und 4.3. 
Kritik Couturats als unhaltbar. ${ }^{6}$ Ein Scheitern der mathematischen Theorie Kants gerade an dem Moment der Intuitivität - so wird gelegentlich betont - müßte die Revision wesentlicher Gehalte der Transzendentalphilosophie, insbesondere der "Transzendentalen Ästhetik" der $K r V$, nach sich ziehen. Kant wird ein unzureichendes Verständis formaler Logik attestiert; zudem habe Kant aus heutiger Sicht noch nicht einmal über ein Konzept der reinen Mathematik (im Sinne Booles) verfügen können. ${ }^{7}$

Es drängt sich allerdings die Frage auf, ob Kant überhaupt eine Theorie der Mathematik geliefert hat oder wenigstens liefern wollte oder der Meinung war, daß er eine solche Theorie liefern könnte. So besteht denn auch Übereinstimmung darüber, daß sich im gesamten Kantischen Euvre keine ausgearbeitete Theorie oder Philosophie der Mathematik findet. Von daher ist es hermeneutisch zumindest unbefriedigend, wenn einzelne (vermeintliche) Fragmente einer solchen Theorie aus verschiedenen Abschnitten der $K r V$ oder anderen Werken isoliert und dann zusammengenommen werden, um auf dieser Basis eine in Ansätzen rekonstruierbare Theorie einer pauschalen $\mathrm{Kritik}^{8} \mathrm{zu}$ unterziehen. Kant selbst hat nur wenige, aber aufschlußreiche Bemerkungen zu dem Projekt einer Philosophie der Mathematik gemacht. Hält er den Versuch, 'über Mathematik zu philosophieren', in der $K r V$ schon für "ein schweres Geschäft!", so wird er später ein solches Unterfangen für unmöglich erklären: "So wenig wie es philosophische Anfangsgründe der Mathematik gibt - eben so wenig kann es mathematische der Philosophie geben, obgleich Newton diese 2 Felder vereinigt." 9

\subsection{Zu Hintikkas Interpretation des Kantischen Konstruktionsbegriffs}

Bei der Bestimmung der mathematischen Erkenntnis als 'Konstruktion der Begriffe' orientiert sich Kant am geometrischen Beweisverfahren Euklids. Diese These Hintikkas (1969) muß jedoch soweit eingeschränkt werden, daß sich die Bedeutung der nach Kant integralen Merkmale mathematischer Erkenntnis - Synthetizität, Intuitivität, Apodiktizität nicht schon durch die vergleichende Analyse mit der historischen Vorlage einfachhin

6 Wer für das Theorem der 'Darstellung in der Anschauung a priori' eine von den 'transzendentalen Grundlehren' (A718 B746) unabhängige mathematikimmanente Lesart zu finden versucht, scheint unweigerlich auf unüberwindliche Schwierigkeiten zu stoßen. Entweder erweist sich die Terminologie, in der Kant über Mathematik spricht, für eine formallogische Interpretation als untauglich. Oder wesentliche Momente der transzendentalen Theorie, z.B. der Konnex von Intuitivität und Rezeptivität, müßten, da mit einer in Teilen rekonstruierten Kantischen Theorie der Mathematik unverträglich, als "grundfalsch" (Hintikka) verworfen werden. Überspitzt formuliert, erweist sich damit Kants Ausformulierung des Konstruktionstheorems als 'Darstellung des Begriffs in der Anschauung a priori' nicht nur als Schwachstelle der Kantischen Theorie selbst, sondern auch als Sollbruchstelle jeder Interpretation dieser Theorie. - Ausgehend von Beth/Hintikka, Parsons und auch Martin hat sich eine rege Debatte über Kants Mathematik- und Konstruktionsbegriff entwickelt. Als wichtige Beiträge seien hier genannt zu Geometrie, Arithmetik und Algebra: Beth (1956/57), Hintikka (1969), Parsons (1969) und (1984), Martin (1972), Young (1982) und (1984), Schirn (1991); zum Konstruktionsbegriff: Peters (1962/66), Mainzer (1970); zum Verhältnis von transzendentaler und mathematischer Methode: Lütterfelds (1975), Hintikka (1984), Büchel (1987).

Couturat (1904) 323.

Ebd. 264.

OP: AA XXI 87. 
erschließen läßt. Es ist vor allem das intuitiv-rezeptive Merkmal, dessen mathematiktheoretische Deutung Hintikka zu einer recht eigenwilligen Folgerung veranlaßt hat: Die kritizistische Prämisse der Kopplung von Anschauung und Sinnlichkeit sei "grundfalsch"10 und zugunsten der logischen Reduktion des Anschauungsmomentes zu verwerfen. Danach sei Anschauung als repraesentatio singularis entsprechend dem begrifflichen Pendant der repraesentatio per notas communes nurmehr als singulärer Term 'im nicht anschaulichen Sinne' bestimmt. ${ }^{11}$

Hintikka will Kants Redeweise von der 'Konstruktion der Begriffe' durch Rekurs auf die Funktionsbestimmung der Schritte im geometrischen Beweis plausibel machen, die unter dem Namen der ekthesis und kataskeue bekannt sind. Am Beispiel von Prop. 20 des ersten Buches der Elemente des Euklid erläutert Hintikka die Funktion der einzelnen Beweisschritte, wobei er zwei der sechs üblichen Schritte ${ }^{12}$ nicht eigens benennt, der Sache nach aber aufführt.

Er nennt: protasis (enunciation), ekthesis (setting-out), kataskeue (preparation, machinery), apodeixis (proof). ${ }^{13}$ Den dihorismos (definition, specification) ordnet er offenbar der ekthesis $\mathrm{zu}$ oder unter. Ob und inwieweit das symperasma (conclusion) als Verallgemeinerung des Schlusses auf der Grundlage des Datums sich von dem partikulären, aber verallgemeinerbaren Ergebnis der apodeixis abheben läßt, geht aus Hintikkas Ausführungen nicht eindeutig hervor.

Hintikka gibt dem Beweisverfahren folgende Interpretation ${ }^{14}$ : In der ekthesis würden die Vorgaben ("content") der protasis auf eine einzelne, noch zu zeichnende Figur angewandt ("apply"). Kant habe genau diesen Schritt vor Augen, wenn er zur Erläuterung seines Konstruktionsbegriffs mit 'Darstellung' "a process analogous to that of mathematical construction" bezeichne. So entspreche ekthesis dem Verfahren, Begriffe in concreto ("in a particular application") zu erwägen. ekthesis und kataskeue seien insofern eng aufeinander bezogen, als die in der ekthesis konstruierte Figur ("the figure constructed in the settingout") noch mit Hilfe gewisser zusätzlicher Punkte, Linien und Kreise vervollständigt ("complete") werde. In beiden Schritten verfahre man dadurch konstruktiv, daß hier neue individuelle geometrische Entitäten in den Beweisgang eingeführt ("introduce") würden. Es sei der durch ekthesis und kataskeue bezeichnete Sachverhalt ("the completion of a figure by means of the introduction of new geometrical entities"15), der den synthetischen

10 Hintikka (1984) 101.

11 Hintikka (1969) 120: "Intuitivity means simply individuality." - Vgl. L §1: AA IX 91 = WW III 521.

12 protasis: "In jedem Dreieck sind zwei Seiten, beliebig zusammengenommen, größer als die letzte." ekthesis: "Das Dreieck sei $A B C . "$

dihorismos: "Ich behaupte, daß in dem Dreieck $A B C$ zwei Seiten, beliebig zusammengenommen, größer sind als die letzte: $B A+A C>B C, A B+B C>A C$ und $B C+C A>A B$."

kataskeue: "Man verlängere $B A$ nach Punkt $D$, mache $A D=C A$ und ziehe $D C$."

apodeixis: "Da $D A=A C$, ist auch $\angle A D C=A C D$ (I,5); also $B C D>A D C$ (Ax. 8); und da $D B C$ ein Dreieck ist mit $\angle B C D>B D C$, dem größeren Winkel aber die größere Seite gegenüberliegt $(\mathrm{I}, 19)$, so ist $D B>B C$. Aber $D A=A C$; also sind $B A+A C>B C$. [...]"

symperasma: [Daher sind in jedem Dreieck zwei Seiten, beliebig zusammengenommen, größer als die letzte - was zu beweisen war.]

13 Hintikka (1969) 126f. Vgl. Heath (1926) I,129-131, Thaer (1980) 420f., Steck (1945) 308-311.

14 Hintikka (1969), 126f.

15 Hintikka (1969) 129. 
Teil des Beweises ausmache. Mit der apodeixis folge dann der Schritt im Beweis, in dem analytisch Schlüsse gezogen würden, soweit sie die bereits eingeführte und vervollständigte Figur beträfen, und zwar unter Bezug auf Axiome, frühere Propositionen und Eigenschaften der konstruierten Figur.

Es ist zunächst darauf aufmerksam zu machen, daß zu den obligatorischen Bestandteilen des Beweises nur protasis, apodeixis und symperasma gehören. ${ }^{16}$ Wenn Hintikka daher ekthesis und kataskeue in den Vordergrund rückt, so ist seine Darstellung des Beweisverfahrens von vornherein auf die Adaption des Kantischen Konstruktionsbegriffs und seine spezielle Deutung hin ausgelegt. Diese Absicht hat ihn (verglichen mit den Darstellungen von Heath und Niebel) offenbar zu einer Reihe von Modifizierungen und Vereinfachungen des Verfahrens veranlaßt, denen nun nachzugehen ist.

Nach Hintikka sind ekthesis und kataskeue zusammengenommen die Schritte im Beweis, denen Kants Konstruktionsbegriff entspricht. Dabei vermag ich jedoch nicht eindeutig zu klären, ob nur ekthesis oder auch kataskeue (als "completion") der Einführung ("introduction") neuer individueller Entitäten in den Beweisgang dient. Ungeachtet dieser ambivalenten Bestimmung soll jedoch schon die ekthesis die eigentliche Konstruktion leisten, so daß Hintikka mit diesem Schritt auch von "the figure constructed in the settingout"17 sprechen kann. Eine von Hintikka hier suggerierte Doppelung des Konstruktionsbegriffs war freilich auch schon den griechischen Geometern geläufig, jedoch in einem anderen Sinne, als Hintikka angibt:

(a) als Unterscheidung von formeller Konstruktion in Gedanken und wirklicher Konstruktion mit Lineal und Zirkel. Dabei bezeichnet die formelle Konstruktion das Verfahren, "mit dessen Hilfe man zu der intendierten Figur gelangt, ohne es nun wirklich auszuführen, etwa in dem Sinne, wie Euklid in der synthesis eines Problems die Schritte der möglichen Konstruktion für den Beweis verwendet." ${ }^{18}$ Gerade und Kreis sind für den systematischen Aufbau der geometrischen Theorie insofern von Bedeutung, als sie als die einfachsten Gebilde zugleich die Mittel sind, von denen bei der Konstruktion auszugehen ist. $^{19}$

(b) als Unterscheidung von Konstruktion (kataskeue) und Zeichnung (anagraphe), wobei das Unterscheidungskriterium in der "Kompliziertheit des methodischen Verfahrens im Gegensatz zur Einfachheit der Zeichnung" gesehen wird. ${ }^{20}$ Die Zeichnung kann durchaus als Bestandteil einer komplizierteren Konstruktion aufgefaßt werden, die wiederum in weitere Teilschritte untergliedert wird. ${ }^{21}$ So spricht Euklid in Prop. 46 zwar von der Zeichnung des Quadrats ${ }^{22}$, doch in Prop. 1 von der Konstruktion des gleichseitigen

16 Heath (1926) I,129.

Hintikka (1969) 126.

Niebel (1959) 9.

Niebel (1959) 28; vgl. $12 \mathrm{f}$.

20 Ebd. 28; vgl. 41f.

21 Ebd. 28: genesis, anagraphe, thesis, parabole, perigraphe, enarmosis, epaphe.

22 Heiberg (1969): "[...] tetragonon anagrapsai." Thaer (1980): "[...] das Quadrat zu zeichnen." Heath (1926): "[...] to describe a square." 
Dreiecks. ${ }^{23}$ Beide Differenzierungen im Verständnis des Konstruktionsbegriffs beziehen sich jedoch nur auf die kataskeue, nicht auch auf die ekthesis und decken sich nicht mit der Unterscheidung, die Hintikka zwischen den Schritten trifft. Welche Funktion der kataskeue im Beweis zukommt, hängt davon ab, ob die Proposition, deren Teil sie ist, einen Lehrsatz (Theorem) oder eine Aufgabe (Problem) formuliert. In den Problemen wird durch die Konstruktion die Existenz der geometrischen Figuren nachgewiesen, die noch nicht im Aufbau der Theorie aufgetreten sind. ${ }^{24}$ Die apodeixis bestätigt dann die korrekte Durchführung der Konstruktion. ${ }^{25}$ Dagegen kommt dem Beweis in den Theoremen die zentrale Rolle zu, die allgemeinen Eigenschaften der bereits als existent nachgewiesenen und daher im folgenden vorausgesetzten Figuren darzulegen. Die kataskeue dient hierbei "höchstens zur Erläuterung". ${ }^{26}$ Die Interdependenz von Problemen und Theoremen im Aufbau der Theorie zeigt sich auch daran, daß umgekehrt gewisse Theoreme erst die notwendigen Bedingungen für die Konstruktion einer Figur angeben, bevor eben die Existenz der Figur durch Konstruktion in einem Problem nachgewiesen wird. Als Beispiel dient hier die von Hintikka benutzte Prop. 20 für Prop. 22. ${ }^{27}$ Es ist festzuhalten, daß kataskeue in Prop. 20 nur eine erläuternde Funktion hat, mithin den 'schwächeren' Konstruktionstyp repräsentiert, nicht jedoch im Vergleich mit der ekthesis, sondern zur existenzsichernden Konstruktion in Prop. 22.

Einen Beleg, der Hintikkas Deutung von ekthesis als geometrische Konstruktion rechtfertigte, habe ich nicht finden können. So liegt die Vermutung nahe, daß ekthesis eine andere Bedeutung haben muß, als ihr Hintikka im geometrischen Beweis und dann auch mit der Interpretation des Kantischen Konstruktionsbegriffs zuweisen will. Eingangs habe ich darauf hingewiesen, daß Hintikka unter dem Namen der ekthesis der Sache nach stillschweigend den dihorismos mitaufführt. Nun treten beide Schritte nur dann im Beweis auf, wenn bereits die Formulierung der Proposition die Informationen enthalten, auf die sie sich in spezifizierter Form beziehen können. Fehlen diese Angaben, so fehlen auch diese beiden Schritte. Schon deshalb ist es irreführend, ekthesis mit der Einführung 'neuer' geometrischer Entitäten gleichzusetzen. ekthesis wiederholt vielmehr nur das in der Proposition genannte Datum ${ }^{28}$ und lautet in Prop. 20 also lediglich: "Das Dreieck sei $A B C . "$ ("For let $A B C$ be a triangle.")

Der dihorismos hat eine doppelte Funktion: entweder bestimmt er das Gesuchte oder die Behauptung näher, oder er legt die Möglichkeit oder Unmöglichkeit der Konstruktion in

23 Heiberg (1969): "[...] trigonon isopleuron systesasthai." Thaer (1980): "[...] ein gleichseitiges Dreieck zu errichten." Heath (1926): "[...] to construct an equilateral triangle." - Vgl. Niebel (1959) 41.

24 Niebel (1959) 39.

25 Ebd. 31.

26 Ebd. 32; vgl. 39.

27 Nach dem Beweis des Theorems (Prop. 20) sind in jedem Dreieck zwei Seiten, beliebig zusammengenommen, größer als die letzte. Das Problem (Prop. 22) gibt auf, aus drei Strecken, die drei gegebenen gleich sind, ein Dreieck zu errichten. Die Bedingungen der Konstruktion formuliert der dihorismos, und zwar mit Rekurs auf Prop. 20: "[...] hierbei müssen [weil in jedem Dreieck zwei Seiten, beliebig zusammengenommen, größer sind als die letzte $(\mathrm{I}, 20)]$ stets zwei, beliebig zusammengenommen, größer sein als die letzte." - Vgl. zur Funktion des dihorismos und zum Verweis auf I,20 Heath (1926) I,130f., 293.

28 Steck (1945) 309f.; Heath (1926) I,132f. 
komplizierten Aufgaben dar. ${ }^{29}$ Unter den im dihorismos spezifizierten Voraussetzungen läßt sich anhand des Datums der in der Proposition geforderte Beweis der Existenz der Figur oder ihrer besonderen Eigenschaften führen. Es sind also die Eigenschaften der Figur und die Bedingungen, unter denen die Figur mit diesen Eigenschaften konstruierbar ist, auf die sich die fraglichen Beweisschritte beziehen. Hintikkas Ausführungen weichen von dieser Analyse deutlich ab.

$\mathrm{Da}$ bei Hintikka Hinweise auf die besonderen Eigenschaften der Figur fehlen, ist um so auffälliger, als sein Beispiel, Prop. 20, als Theorem gerade die Eigenschaften beweisen soll, die zur Konstruktion der Figur in Prop. 22 notwendig sind. Folgerichtig führt Hintikka denn auch den Wortlaut der apodeixis nicht eigens auf. Dagegen hebt Hintikka die Partikularität der Figur hervor und vermeidet geradezu, den Bezug der ekthesis, das Datum, zu nennen. Es wird sich zeigen, daß gerade das hier Datum Genannte in der transzendentallogischen Interpretation sich durch das intuitiv-rezeptive Moment des Anschauungsbegriffs, d.h. der Gegebenheitsweise von Mannigfaltigem, bezeichnen läßt. Hintikkas Interpretationsstrategie leuchtet freilich nur dann ein, wenn man seine Lesart von Anschauung als individueller Entität, abgekoppelt vom Vermögen der Sinnlichkeit, berücksichtigt.

Ich stimme mit Hintikka darin überein, daß Kant mit dem euklidischen Beweisverfahren in seinen konstruktiv-synthetischen wie deduktiv-analytischen Teilen durchaus vertraut ist. Allerdings verstellt Hintikkas Absicht, dem Kantischen Konstruktionstheorem eine logische Deutung zu geben, bisweilen den Blick auf die Funktion einzelner Beweisschritte und deren Interpretation durch Kant. Insbesondere Hintikkas Kernthese, daß das Konzept von Konstruktion als 'Darstellung in der Anschauung' in irgendeinem Sinn dem ekthesis genannten Schritt entspreche ${ }^{30}$, ist nicht haltbar. Nach den Ausführungen von Heath und Niebel läßt sich ekthesis weder terminologisch noch der Funktion im geometrischen Beweis nach als Konstruktion verstehen. Einerseits ist Konstruktion auf den Schritt der kataskeue beschränkt, der freilich die interne Unterscheidung von imaginierter und aktueller Konstruktion oder von (komplizierter) Konstruktion und (einfacher) Zeichnung zuläßt. Andererseits erfüllt ekthesis im Beweis eine andere Funktion, als ihr Hintikka zuschreibt. Daß ekthesis eigentlich das Datum (für die Konstruktion) angibt, vergißt Hintikka zu erwähnen.

Aus der Anmerkung zum Lehrsatz 1 der MADyn geht hervor, daß Kant mit der auch durch Heath beschriebenen Funktion der ekthesis durchaus vertraut ist: Der Mathematiker könne "von jedem beliebigen Dato seine Konstruktion eines Begriffes anfangen"; er habe z.B. mit dem Begriff der Solidität "etwas als ein erstes Datum der Konstruktion des Begriffs einer Materie [...] angenommen." ${ }^{31}$ Wenn mit dem Datum die Konstruktion begonnen werden kann, so ist die ekthesis zwar die Voraussetzung, nicht jedoch schon der Anfang oder ein Teil der Konstruktion.

\footnotetext{
29 Niebel (1959) 29; vgl. 31.

30 Hintikka (1969) 126: "the figure constructed in the setting-out".

31 MANW: AA IV $498=$ WW V 49.
} 


\subsection{Kants transzendentallogische Deutung des euklidischen Beweisverfahrens}

Im Anschluß an die Detailkritik der Ausführungen Hintikkas versuche ich eine alternative Interpretation der Kantischen Lesart des geometrischen Beweismodells nach Euklid zu entwickeln. Einleitend werde ich einige Rahmenbedingungen angeben, unter denen die Differenz von philosophischer und mathematischer Erkenntnisart verständlich gemacht werden kann.

Im Unterschied zur philosophischen Erkenntnis als 'Erkenntnis aus Begriffen' kennzeichnet Kant mathematische Erkenntnis als 'Konstruktion der Begriffe' durch die Darstellung des Begriffs in einer dem Begriff korrespondierenden Anschauung a priori. (A713 B741). Abgesehen von der notorischen Unklarheit der Bestimmung mathematischer Erkenntnis wirft diese Formel, so griffig sie zunächst auch erscheinen mag, besondere Schwierigkeiten auch bei der Explikation philosophischer Erkenntnis auf. Denn entgegen einer naheliegenden Deutung kann 'Erkenntnis aus Begriffen' nach den epistemologischen Vorgaben der $\mathrm{KrV}$ weder auf ein Verfahren logischer Begriffsanalyse reduziert noch, als Erkenntnis, unbezogen auf Anschauungsgehalte formuliert werden.

Als Kriterium der Unterscheidung beider Arten begriffsbezogener Erkenntnis gibt Kant zwar 'Konstruktion' an; doch bleibt zunächst unklar, wie denn die Beziehung beider Erkenntnisarten präzisiert werden muß, damit deren Differenz gerade durch das 'Darstellung in der Anschauung' genannte Kriterium auch explizierbar ist. Weder wäre die Analytizität formallogischer Sätze solch ein hinreichendes Unterscheidungskriterium, da Kant dem Mathematiker den Gebrauch synthetischer wie auch analytischer Sätze, sofern in der Anschauung a priori darstellbar, zugesteht (B16). Noch bildete das intuitiv-rezeptive Merkmal mathematischer Erkenntnis einen nicht-trivialen Unterschied zu einer formallogischen Disziplin, da hiermit ohnehin Inkommensurables und in der Folge Unvermittelbares voneinander abgegrenzt würde.

Es ist denn auch Kant selbst, der die begriffsanalytische Reduktion philosophischer Erkenntnis ausschließt und darauf hinweist, daß die eigentümliche Differenz beider Erkenntnisarten erst auf der Folie der in der $\mathrm{KrV}$ "vorgetragenen transzendentalen Grundlehren" erhelle:

"Es kommt hier nicht auf analytische Sätze an, die durch bloße Zergliederung der Begriffe erzeugt werden können, (hierin würde der Philosoph ohne Zweifel den Vorteil über seinen Nebenbuhler haben,) sondern auf synthetische, und zwar solche, die a priori sollen erkannt werden." (A718 B746)

Grundlage der Differenzierung philosophischer und mathematischer Erkenntnis ist die Klasse synthetischer Sätze a priori, wobei gelten soll, daß beide Erkenntnisarten sich gerade nicht in der "Allgemeinheit der Erkenntnis und ihrer Erzeugung a priori" unterscheiden, jedoch - wie es in A723 B751 heißt - "im Fortgange sehr verschieden" sind. Es kommt nun darauf an, im Hinblick auf die 'transzendentalen Grundlehren' den systematischen Ort ausfindig zu machen, an dem sich die Differenz der Erkenntnisarten, offensichtlich ungeachtet ihrer Geltung und Erzeugung, "im Fortgange" manifestiert.

Erkenntnisse vom Typ synthetischer Sätze a priori beziehen sich auf solche Eigenschaften des durch den Begriff bezeichneten Gegenstandes, die als Merkmale zum Begriff gehören, 
nicht aber durch die logische Analyse des Begriffsinhalts bestimmbar sind. ${ }^{32}$ Im Urteil werden diese Begriffe insofern auf Anschauungen bezogen, als die fraglichen Eigenschaften entweder in der reinen Anschauung oder empirischen Anschauung 'in concreto dargestellt', d.h. gegeben werden, und so entweder die apriorische, d.h. mathematische Erkenntnis, oder aposteriorische, d.h. empirische Erkenntnis dessen ermöglichen, 'was dem Gegenstand des Begriffs zukommt'. (A721 B750)

Philosophische Erkenntnis verhält sich nun zu der Dublette der in concreto darstellbaren Fälle von Erkenntnis so, (a) daß sie die Bedingungen vorgibt, unter denen empirische Erkenntnis erst möglich und formulierbar ist, und dies näher so, (b) daß der Wahrheitswert empirischer Erkenntnis entscheidbar sein muß und (c) daß empirische Erkenntnis den Bedingungen physikalischer und mathematischer Beschreibung genügt.

Reflektiert man auf die transzendentalen Momente 'Form' und 'Materie' empirischer Erkenntnis, so geben die mathematischen Sätze hinsichtlich 'Gestalt', 'Dauer' und 'Zahl' die formale Bestimmung des als Erscheinung interpretierten Falls empirischer Erkenntnis in der reinen sinnlichen Anschauung. (A724 B758) Von mathematischen Sätzen als Erkenntnissen spricht Kant nur, "sofern man voraussetzt, daß es Dinge gibt, die sich nur der Form jener reinen sinnlichen Anschauung gemäß uns darstellen lassen." (B147) Diese Erkenntnisbedingung setzt folglich die Geltung solcher Sätze voraus, die im Hinblick auf die kategorial vermittelte Konstitution von Dingen die materiale Bestimmung empirischer Erkenntnis ermöglichen. (A724 B752) Solche genuin philosophischen und transzendental genannten Sätze formulieren nämlich

\footnotetext{
"die Regel, nach der eine gewisse synthetische Einheit desjenigen, was nicht a priori anschaulich vorgestellt werden kann, (der Wahrnehmungen,) empirisch gesucht werden soll. Sie können aber keinen einzigen ihrer Begriffe a priori in irgendeinem Falle darstellen, sondern tun dies nur a posteriori, vermittelst der Erfahrung, die nach jenen synthetischen Grundsätzen allererst möglich wird." (A720f. B748f.)
}

Dem Kantischen Sprachgebrauch folgend, heißt dies, daß transzendentale Begriffe einer Synthesis empirischer Anschauungen der Darstellung a posteriori bedürfen. Vor diesem Aufriß einzelner Lehrstücke der Kantischen Theorie der Erfahrung lassen sich die Rahmenbedingungen für eine Interpretation des Konstruktionstheorems näher bestimmen:

(a) Setzt man voraus, daß sich Kant bei der Bestimmung mathematischer Erkenntnis als 'Konstruktion der Begriffe' tatsächlich am euklidischen Beweisverfahren orientiert, und setzt man ferner voraus, daß diese Bestimmung erst im Rekurs auf die in der $\mathrm{Kr} V$

32 Eberhard bezeichnet diese synthetischen Merkmale des Begriffs als Attribute, die anders als die analytischen Merkmale nicht nach dem Satz des Widerspruchs, sondern nach dem Satz des Grundes erkennbar sein sollen. Kant weist in der Streitschrift gegen Eberhard darauf hin, daß sich nach Eberhard der Unterschied analytischer und synthetischer Urteile a priori nur insoweit benennen lasse, "als daß das Prädikat derselben notwendig im Wesen des Begriffs des Subjekts auf irgend eine Art gegründet, mithin Attribut sei, aber nicht bloß zufolge des Satzes des Widerspruchs. Wie es nun aber, als synthetisches Attribut, mit dem Begriff des Subjekts in Verbindung komme, da es durch Zergliederung desselben daraus nicht gezogen werden kann, ist aus dem Begriffe eines Attributs und dem Satze: daß irgend ein Grund desselben sei, nicht zu ersehen [...]. Die Kritik aber zeigt den Grund der Möglichkeit desselben deutlich an, nämlich: daß es reine, dem Begriffe des Subjekts untergelegte Anschauung sein müsse, an der es möglich, ja allein möglich ist, ein synthetisches Prädikat a priori mit einem Begriffe zu verbinden." (E: AA VIII 241ff. = WW III 362ff.) 
'vorgetragenen transzendentalen Grundlehren' verständlich wird, dann ist die Annahme berechtigt, daß die Formulierung des Konstruktionstheorems eine Interpretation des geometrischen Beweisverfahrens in der Terminologie und systematischen Perspektive transzendentaler Logik gibt.

(b) Wenn die transzendentale Logik die Bedingungen einer Theorie der Erfahrung und genauer einer apriorischen Theorie der empirischen Wissenschaft von der Natur (Physik) enthält, mithin auch die Anwendung der Mathematik auf den Objektbereich einer solchen Wissenschaft zu begründen sucht, dann steht die Formulierung des Konstruktionstheorems in Abgrenzung zur philosophischen Erkenntnis auch unter den speziellen Bedingungen dieses Begründungsprogrammes.

(c) Wenn Kant daher behauptet, "daß Meßkunst und Philosophie zwei ganz verschiedene Dinge seien, ob sie sich zwar in der Naturwissenschaft einander die Hand bieten" (A726 B754), so folgt, daß Mathematik und Philosophie zwar nicht logisch aufeinander reduzierbar oder voneinander ableitbar, doch in der Perspektive der philosophisch begründeten Anwendung der Mathematik auf empirische Objekte vermittelbar sind.

(d) Nach der Formation der in der $\mathrm{KrV}$ entwickelten Propädeutik der Kantischen Theorie der Erfahrung setzt die Geltung mathematischer Sätze über den Objektbereich empirischer Wissenschaften die transzendental deduzierte Geltung der Kategorien für objektive Gegenstände bereits voraus. Wie ich zeigen möchte, erhellt die Differenz der Erkenntnisarten genauer aus dem Nachweis der objektiven Realität von Begriffen in mathematischen und transzendentalen Sätzen. Der Nachweis der objektiven Realität von Begriffen ist der Nachweis, daß die Objekte, die ich durch diese Begriffe denke und bezeichne, durch mich und jeden anderen als Objekte der (naturwissenschaftlich beschreibbaren) Erfahrung bestimmt und erkannt werden können.

(e) Wenn sich die Differenz von philosophischer und mathematischer Erkenntnisart anhand des Nachweises der objektiven Realität der Begriffe einsichtig machen läßt, so ist diese Differenz schon dem transzendentalphilosophischen Begründungsprogramm von Erfahrung immanent; d.h. die Differenz ergibt sich aus der Bestimmung der jeweiligen Funktion, die mathematische und philosophische Erkenntnisart bei der Konstituierung und Regulierung von Erfahrung haben. Nun behauptet Kant, daß die objektive Realität der Begriffe a priori zwar "unabhängig von der Erfahrung, aber doch nicht unabhängig von aller Beziehung auf die Form einer Erfahrung überhaupt, und die synthetische Einheit, in der allein Gegenstände empirisch können erkannt werden", nachweisbar sei. (A221 B269) Die fragliche Funktion der Erkenntnisarten ist insofern bestimmbar, als die 'mathematischen Grundsätze' das Objekt der Erfahrung als Erscheinung in seiner Gegebenheitsweise als Größe konstituieren, währenddessen die 'dynamischen Grundsätze' die Suche nach der materiellen Bestimmung des Objekts regulieren. (A179f. B222f.)

(f) Die Differenz beider Erkenntnisarten zeigt sich an der direkten (apriorischen, da durch die konstitutiven Prinzipien der Axiome und Antizipationen bestimmten) und indirekten (aposteriorischen, da durch die bloß regulativen Prinzipien der Analogien bestimmten) Darstellbarkeit der Begriffe und ist, wie angedeutet, durch den Rekurs auf den unterschiedlichen Prinzipientyp oder "Bestimmungsgrund" begründet. Dies ist der Sinn 
eines Schlüsselzitats, mit dem Kant die Benennung der Differenz beider Erkenntnisarten begründet: "Das Erkenntnis durch Begriffe heißt diskursiv, das in der Anschauung intuitiv; in der Tat wird zu einer Erkenntnis beides mit einander verbunden erfordert, sie wird aber von dem benannt, worauf, als den Bestimmungsgrund desselben, ich jedesmal vorzüglich attendiere." ${ }^{33}$

(g) Nun sind die genannten mathematischen und dynamischen Prinzipien als transzendentale Grundsätze des Verstandes nicht isoliert wirksam, sondern gerade im Nachweis der objektiven Realität in der Hinordnung auf das Konzept der transzendentalen Wahrheit der 'Einen Erfahrung'. So besagt der Nachweis der objektiven Realität z.B. von Begriffen einer geometrischen Figur, daß sie sich deshalb "auf mögliche Dinge" beziehen, "weil sie die Form der Erfahrung überhaupt a priori in sich enthalten." (A221 B268) Für Begriffe wie Ursache und Wirkung stellt Kant entsprechend fest: "Nur daran also, daß diese Begriffe die Verhältnisse der Wahrnehmungen in jeder Erfahrung a priori ausdrücken, erkennt man ihre objektive Realität, d.i. ihre transzendentale Wahrheit". (A221f. B269)

(h) Die Hinordnung der Prinzipien auf das Konzept der transzendentalen Wahrheit vermitteln die 'Postulate des empirischen Denkens überhaupt'. Sie erlauben die Umsetzung dieses Konzepts, d.h. die Ermittlung und Überprüfung empirischer Wahrheit. ${ }^{34}$ Dieser Sachverhalt wird, ausgehend von den MAPhän, im Zuge der Interpretation des Konstruktionsbegriffs in den $M A N W$ (5.1. - 5.3.) erläutert.

Auf der Grundlage der in 4.2. vorgestellten Analyse des euklidischen Beweisverfahrens soll nun eine zu Hintikka alternative Interpretation des Kantischen Konstruktionstheorems entwickelt werden. Dabei gehe ich von der Textstelle A715f. B743f. aus:

\footnotetext{
Die Mathematik "kann mit dem bloßen Begriffe nichts ausrichten, sondern eilt sogleich zur Anschauung, in welcher sie den Begriff in concreto betrachtet, aber doch nicht empirisch, sondern bloß in einer solchen, die sie a priori darstellt, d.i. konstruiert hat, und in welcher dasjenige, was aus den allgemeinen Bedingungen der Konstruktion folgt, auch von dem Objekte des konstruierten Begriffs allgemein gelten muß."
}

Die sprachliche und argumentative Verfassung der Textstelle ist auf den ersten Blick irritierend, bietet sie doch jene eigentümliche Mischung einer teils metaphorischen, teils formelhaften Redeweise und einer Satzstruktur, die den syntaktisch vorgegebenen Bezügen einzelner Satzglieder der Sache nach nicht einwandfrei folgt. So gibt der Wortlaut des Textes, was den Gegenstand der Konstruktion anlangt, keine eindeutige Auskunft: Der Mathematiker betrachte in der Anschauung "den Begriff in concreto", doch ist es eben diese Anschauung, die er "a priori darstellt, d.i. konstruiert hat"; zugleich wird "von dem Objekte des konstruierten Begriffs" gesprochen. Zudem scheint Kant die apriorische Darstellung von etwas als Resultat seiner Konstruktion oder zumindest als bereits durchgeführte Konstruktion ("darstellt, d.i. konstruiert hat") aufzufassen.

$33 F$ : AA XX $325=$ WW III 666.

34 Vgl. A146 B185: "In dem Ganzen aller möglichen Erfahrung liegen aber alle unsere Erkenntnisse, und in der allgemeinen Beziehung auf dieselbe besteht die transzendentale Wahrheit, die vor aller empirischen vorhergeht, und sie möglich macht." 
Wie aus dem Kontext des Zitats hervorgeht, will Kant den Unterschied von philosophischer und mathematischer Betrachtung von Begriffen an der Art des Gebrauchs von Begriffen in Urteilen festmachen. Diesen entscheidenden Punkt vermag er hier jedoch nur metaphorisch auszudrücken, wonach eben 'der Mathematiker mit dem bloßen Begriff nichts ausrichten könne und sogleich zur Anschauung eile'. Diese Redeweise legt die Vermutung nahe, daß die Differenz der Erkenntnisarten an der Art, wie Begriff und Anschauung jeweils aufeinander bezogen werden, deutlich wird. Während der Mathematiker 'sogleich zur Anschauung eile', bleibt dem Philosophen dieses Verfahren, Begriff und Anschauung direkt in Beziehung zu setzen, versagt.

Denn Kant behauptet nicht, daß der Philosoph nur 'mit dem bloßen Begriff' hantiere; allerdings vermag er, nun seinerseits im Vorteil, auch schon 'mit dem bloßen Begriff' etwas 'auszurichten'. Kant behauptet auch nicht, daß philosophische Urteile in jedem Fall unbezogen auf Anschauungen gefällt werden. Im Gegenteil, sein Anliegen war es ja gerade, zu zeigen, daß diese Urteile nur dann den Kriterien wissenschaftlicher Erkenntnisse entsprechen, wenn ihr Gebrauch auf eine Mannigfaltigkeit von Anschauungen zum Zwecke möglicher Erfahrung restringiert wird. Diese Beziehung von Begriff und Anschauung in transzendentalen Sätzen wird jedoch nur 'im nachhinein' vollzogen und nicht, wie Kant sich ganz eigentümlich ausdrückt, "sogleich" oder "sofort" (A711 B739, A716 B744) oder "unmittelbar" (A732 B760).

Es ist das Charakteristikum transzendentaler Erkenntnis, daß sie sich auf das, worüber sie urteilt, "nicht geradezu" (A782 B810) bezieht, sondern zunächst die Rechtmäßigkeit dieser Beziehung und die Bedingungen der Realisierung der Beziehung nachzuweisen ist. Kant spricht von der Forderung, "zuvor die objektive Gültigkeit der Begriffe und die Möglichkeit der Synthesis derselben a priori" darzutun. (A782 B810)

Im Unterschied zur Darstellung a priori mathematischer Begriffe lassen sich auch die transzendentalen Begriffe "darstellen", jedoch "nur a posteriori, vermittelst der Erfahrung" (A720f. B748f.), wobei eben diese Erfahrung erst durch die transzendentalen Begriffe konstituiert und machbar wird, in dem Sinne, daß dieses hier Erfahrung Genannte genau dasjenige ist, "was [...] empirisch gesucht werden soll." (A720f. B748f.) Kant muß diesen Sachverhalt der Darstellbarkeit von Begriffen vor Augen haben, wenn er im folgenden Text den zweiten Differenzpunkt so bestimmt: "[...] Anschauung, in welcher sie den Begriff in concreto betrachtet, aber doch nicht empirisch, sondern bloß in einer solchen, die sie a priori darstellt, d.i. konstruiert hat [...]."

Es fällt auf, daß Kant bei der Unterscheidung der Erkenntnisarten vorderhand mathematische von empirischer Erkenntnis abgrenzt, geht man einmal davon aus, daß 'empirische Darstellung' sich auf empirische, 'apriorische Darstellung' auf reine Anschauung bezieht. Ich sehe zunächst von der näheren Bestimmung des Anschauungsbegriffs ab und möchte auf einige Implikate des Konstruktionsbegriffs im folgenden Satzteil hinweisen. Denn dort scheint Kant die Bestimmungen zum Konstruktionsbegriff direkt zu benennen, ohne eigens den Unterschied zur philosophischen Erkenntnisart zu thematisieren:

"[...] Anschauung [...] in welcher dasjenige, was aus den allgemeinen Bedingungen der Konstruktion folgt, auch von dem Objekte des konstruierten Begriffs allgemein gelten muß." 
Kant unterscheidet Objekt und Begriff des Objekts, wobei nicht das Objekt, sondern der Begriff des Objekts konstruiert wird. Nun behauptet Kant nicht, daß die Bedingungen der Konstruktion des Begriffs auch von dem Objekt des Begriffs allgemein gelten müssen; vielmehr muß von dem Objekt des konstruierten Begriffs allgemein gelten genau "dasjenige, was aus den allgemeinen Bedingungen der Konstruktion folgt". Was sind die Bedingungen der Konstruktion, und welche Folgerungen mit Anspruch auf Allgemeingültigkeit lassen sich daraus ableiten? Bei der Klärung dieser Fragen sind die Struktur des gesamten Arguments und die Perspektive der Differenzierung zu berücksichtigen. Nach der Logik des Vergleichs muß Kant hier gerade von etwas sprechen, was die transzendentale Synthesis in Grundsätzen im Gegensatz zur mathematischen nicht leistet. Dazu führt er an einer Parallelstelle in den $M A N W$ folgendes aus:

"Zur Konstruktion der Begriffe wird erfodert: daß die Bedingung ihrer Darstellung nicht von der Erfahrung entlehnt sei [...] oder überhaupt, daß die Bedingung der Konstruktion nicht selbst ein Begriff sein müsse, der gar nicht a priori in der Anschauung gegeben werden kann". ${ }^{35}$

Die Textstelle stimmt mit den Beobachtungen zur Sache wie zum Argumentationszug (A715f. B743f.) überein: Kant charakterisiert die mathematische Konstruktion durch negative Bestimmungen im Vergleich zur Darstellung des transzendentalen Begriffs einer Synthesis. ${ }^{36}$ Im weiteren Text stellt Kant denn auch klar, daß solche Bedingungen nicht Begriffe von "Ursache und Wirkung, Handlung und Widerstand etc." sein können, sondern wie im Fall der Phoronomie "Konstruktion der Bewegungen überhaupt als Größen". ${ }^{37}$

Der Begriff der Ursache ist nicht konstruierbar, jedoch nicht deshalb, weil er 'aus der Erfahrung entlehnt' sei; denn als Kategorie gehört er zu den Begriffen, die 'vor aller Erfahrung' als deren Bedingung diese allererst möglich machen. Nach dem Argument ist er nicht konstruierbar, weil die Bedingung seiner Darstellung 'aus der Erfahrung entlehnt' ist. Es wird sich zeigen, daß es eben die Aposteriorität seiner Darstellung ist, die seine Konstruktion ausschließt.

Kant bestimmt Mathematik traditionell als 'Größenlehre'. Nach der Bestimmung mathematischer Erkenntnis als 'Konstruktion der Begriffe' sind Begriffe also nur dann konstruierbar, wenn man sie als Größen betrachten kann. Kant fährt fort:

\footnotetext{
"Hier ist nun vorzüglich zu bemerken: daß Phoronomie durchaus zuerst Konstruktion der Bewegungen überhaupt als Größen, und, da sie Materie bloß als etwas Bewegliches, mithin an welchem gar auf keine Größe derselben Rücksicht genommen wird, zum Gegenstande hat, diese Bewegungen allein als Größen, so wohl ihrer Geschwindigkeit als Richtung nach, und zwar ihrer Zusammensetzung nach a priori zu bestimmen habe. Denn so viel muß gänzlich a priori und zwar anschauend zum Behuf der angewandten Mathematik ausgemacht werden. Denn die Regeln der Verknüpfung der Bewegungen durch physische Ursachen, d.i. Kräfte, lassen sich, ehe die Grundsätze ihrer Zusammensetzung überhaupt vorher rein mathematisch zum Grunde gelegt worden, niemals gründlich vortragen." ${ }^{38}$
}

Als physikalisch-mathematische Teildisziplin der Mechanik betrachtet die Phoronomie oder Kinematik Bewegungen von Körpern, wobei eben von der räumlichen Ausdehnung 
und Gestalt des Körpers abgesehen wird und man sich seine Masse in einem Punkt vereinigt denkt. Dieser Massenpunkt ist ein Modell des Körpers. Daher spricht man genauer von "Bewegungen von Massenpunkten, sofern von hierbei auftretenden Kräften abgesehen wird." ${ }^{39}$ Sofern die Phoronomie eine 'rein mathematische Grundlegung' sein soll, folgt Kant dieser klassischen Bestimmung. Er versteht die Phoronomie ja als "die reine Größenlehre (mathesis) der Bewegungen"40. Bewegung wird als ein reines Quantum $^{41}$, die Materie unabhängig vom Begriff der Ausdehnung als ein Punkt betrachtet. ${ }^{42}$ Kant geht es nun darum, zu dieser Phoronomie genannten physikalischmathematischen Disziplin die 'metaphysischen Anfangsgründe' zu formulieren. Das, was die empirische Grundbestimmung des Begriffs der Materie ausmacht, soll mit Bezug auf metaphysische Prinzipien so gedacht werden, daß der Begriff der Bewegung 'zum Behuf der angewandten Mathematik' exponibel wird.

Die Art, wie Kant diese Aufgabenstellung exponiert, gibt Aufschluß darüber, in welchem Kontext und Sinn überhaupt von Konstruierbarkeit die Rede sein kann. Überraschend ist dabei, nicht daß, sondern wie Kant die beiden Momente der Erwägung des Bewegungsbegriffs, Geschwindigkeit und Richtung, einbeziehen will. Richtung bzw. Richtungsänderung sind dann zu berücksichtigen, wenn man Beschleunigung bzw. Geschwindigkeitsänderungen berechnen will. Kant legt nun besonderen Nachdruck auf den Sachverhalt, daß MAPhor "durchaus zuerst" Konstruktion der Bewegungen als Größen, "und zwar ihrer Zusammensetzung nach a priori", noch genauer, "gänzlich a priori und zwar anschauend" sei. Diesem Kriterium der Darstellungsweise könnte er nicht entsprechen, wenn er nicht die "Zusammensetzung der Bewegung und zwar von der Möglichkeit der gradlinichten Bewegung allein, nicht der krummlinichten" einbezogen hätte. ${ }^{43}$ Es ist erst diese Beschränkung der Zusammensetzung (ausschließlich der gradlinichten Bewegung), die "die Bestimmung aller möglichen Bewegung als eines Quantum" (als Datum) erlaube, "wiewohl die Quantität derselben (an einem beweglichen Punkte) bloß in der Geschwindigkeit besteht." 44

Sofern die Einwirkung von Kräften bei der Berechnung von Beschleunigungen bei Kant nicht berücksichtigt werden soll, bekommt das Moment der Richtung noch einen anderen Sinn; daran soll nämlich die Dualität entgegengesetzter Grundkräfte (mathematisierbar) einsichtig gemacht werden. ${ }^{45}$ Mit der Unterscheidung von Quantum und Quantität macht Kant auf den transzendentalen Grundlegungskontext aufmerksam. ${ }^{46}$ Kant geht es hier um

39 Leute (1972) 8.

40 MANW: AA IV 489, $495=\mathrm{WW}$ V 37, 45f.

41 MANW: AA IV 477, $495=\mathrm{WW}$ V 22, 46.

42 MANW: AA IV 482, 489 = WW V 27,37. - Vgl. AA IV $480=$ WW V 25: "Wenn gleichwohl der Ausdruck eines Körpers hier bisweilen gebraucht werden sollte, so geschieht dies nur, um die Anwendung der Prinzipien der Phoronomie auf die noch folgende bestimmtere Begriffe der Materie gewissermaßen zu antizipieren".

43 MANW: AA IV $495=\mathrm{WW}$ V 45.

44 MANW: AA IV $495=\mathrm{WW}$ V 46.

45 Vgl. 5.7.

46 MANW: AA IV 495 = WW V 46. - Böhme (1986) 74: "Quanta sind Entitäten, sofern sie als so und so groß bestimmt sind, Quantität ist der Titel des Begriffes, nach dem etwas bestimmt sein muß, um so und so groß genannt werden zu können." 
die Frage, wie Geschwindigkeit als intensive Größe räumlich dargestellt werden kann; die Frage sei "lediglich [...], wie der Begriff der Geschwindigkeit als eine Größe [AA IV 494 abweichend: einer Größe] konstruieret werde". ${ }^{47}$

Diese zunächst recht unscheinbare Konjektur verschüttet jedoch eine alternative Lesart des hier exponierten Problems: Es gilt zu klären, ob der Gegenstand der Konstruktion 'der Begriff einer Größe' ist, als der irgend etwas betrachtet werden soll, oder eben diese 'Größe selber', verstanden als Datum für die Konstruktion. Die Konjektur legt die Lesart nahe, daß der Begriff der Geschwindigkeit 'als einer Größe' konstruiert werden soll; d.h. Geschwindigkeit wird schon als Größe betrachtet, deren Begriff dann konstruiert werden soll. Warum gilt nun die Kategorie der Quantität (und auch der Qualität) als konstruierbar, während die Kategorie der Relationsklasse (und auch der Modalität) sowie die davon abgeleiteten Prädikabilien vom Verfahren der Konstruktion ausgeschlossen sind? Es ist oben deutlich geworden, woran sich die Differenz der Erkenntnisarten zeigt: offenbar an den unterschiedlichen Bedingungen der Darstellung der Verstandesbegriffe, d.h. ihrer Darstellung a posteriori bzw. a priori. Offen bleibt dabei aber die Frage, worin der Grund der Differenz liegt, die sich am unterschiedlichen Rekurs auf die Anschauungstypen zeigt.

Es war bekanntlich Humes Zweifel an der Geltung des Kausalitätsprinzips, der Kant aus dem 'dogmatischen Schlummer' gerissen hatte. Sofern dieses Prinzip besagt, "daß die Wirkung nicht bloß zu der Ursache hinzukomme, sondern durch dieselbe gesetzt sei, und aus ihr erfolge" (A91 B124), bleibt die Notwendigkeit dieser Weise der Verknüpfung von Ursache und Wirkung so lange 'unbegreiflich' (A113), als man nicht erklären kann, wie das, was uns da als Faktum begegnet, so konstituiert wird, wie wir es auffassen. Kant ließ es sich seinerseits denn auch nicht nehmen, am Beispiel der kosmologischen Idee der "Verknüpfung des Bedingten mit seiner Bedingung" eben "das merkwürdigste Phänomen" zu präsentieren, um nun seinerseits "die Philosophie aus ihrem dogmatischen Schlummer zu erwecken und sie zu dem schweren Geschäfte der Kritik der Vernunft selbst zu bewegen." 48

Gemäß seiner Ausgangsfrage, wie Erfahrung möglich sei (1.2.), geht es Kant darum, diese Art der dynamischen Verknüpfung, die wir durch das Verhältnis von Ursache und Wirkung bezeichnen, da, wo sie in der Erfahrung angetroffen wird, zu verstehen" (A770 B798). Es gilt, wie Kant sagt, 'höherzusteigen' oder auch: "meine Begriffe von einem Gegenstand bis zur transzendentalen Bedeutung" zu 'steigern' (A190 B235). Es heißt, nicht mehr wie der Physiker den Gegenstand als 'Ding an sich' zu betrachten, sondern als 'Erscheinung'. Die $K r V$ liefert mit der transzendentalen Fragestellung den methodischen Ansatz, mit dem es gelingen soll, die Welt als den Raum dessen, in dem wir Erfahrung machen, unter der Prämisse, daß wir es dort nur mit Erscheinungen zu tun haben, zu erschließen. Grundlage dafür ist eine Sichtweise, nach der Erfahrungserkenntnis "ein Zusammengesetztes aus dem sei, was wir durch Eindrücke empfangen, und dem, was unser eigenes Erkenntnisvermögen (durch sinnliche Eindrücke bloß veranlaßt) aus sich selbst hergibt" (B1f.) Diese Zusammensetzung lasse sich nun durch die Technik der 
transzendentalen Reflexion (B316ff. 324ff.) und "lange Übung" in seine Bestandstücke zerlegen. Kant spricht hier immer wieder von einer "Isolierung" nach gewissen Hinsichten, also den Elementen der Sinnlichkeit (A22 B36) und des Verstandes (A62 B87; vgl. A842 B870).

Es ist diese Sichtweise, die es ihm erlaubt, "Welt" einerseits als "das mathematische Ganze aller Erscheinungen" zu betrachten. Dieser Weltbegriff kann folglich dem Kalkül unterworfen werden, da man hierbei nur "auf die Aggregation im Raume oder der Zeit, um sie als eine Größe zustande zu bringen [...], sieht." (A418f. B446f.) Andererseits soll gelten: "Eben dieselbe Welt wird aber Natur genannt, sofern sie als dynamisches Ganzes betrachtet wird, und man [...] auf die Einheit im Dasein der Erscheinungen sieht. Da heißt nun die Bedingung von dem, was geschieht, die Ursache". (ebd.) Dabei ist das Verhältnis von Ursache und Wirkung in jedem Fall "der Zeit nach bestimmbar" (A203 B248), d.h. nach dem Kausalitätsprinzip kann das Dasein der Erscheinungen a priori unter Regeln gebracht werden, indem jeder Erscheinung die Stelle in der Zeit nach den Zeitmodi der Beharrlichkeit, der Folge und des Zugleichseins angewiesen wird. ${ }^{49}$ Diese Zeitfolge ist nun gerade "das einzige empirische Kriterium der Wirkung, in Beziehung auf die Kausalität, die vorhergeht." (A203 B249)

Die Analogien der Erfahrung formulieren damit eine Regel, "es [das empirische Kriterium] in der Erfahrung zu suchen, und ein Merkmal, es in derselben aufzufinden." (A179f. B222f.) Diese Prinzipien sind insofern bloß regulativ und nicht konstitutiv, als sie die Verknüpfung von Ursache und Wirkung nicht danach bestimmen, "welche andere und wie große Wahrnehmung, sondern, wie sie dem Dasein nach, in diesem modo der Zeit, mit jener notwendig verbunden sei." In diesem Zusammenhang kann die Differenz von philosophischer und mathematischer Erkenntnisart durch folgendes Zitat verdeutlicht werden:

\footnotetext{
"Vermittelst des Begriffs der Ursache gehe ich wirklich aus dem empirischen Begriffe von einer Begebenheit (da etwas geschieht) heraus, aber nicht zu der Anschauung, die den Begriff der Ursache in concreto darstellt, sondern zu den Zeitbedingungen überhaupt, die in der Erfahrung dem Begriffe der Ursache gemäß gefunden werden möchten. Ich verfahre also bloß nach Begriffen, und kann nicht durch Konstruktion der Begriffe verfahren, weil der Begriff eine Regel der Synthesis der Wahrnehmungen ist, die keine reine Anschauungen sind, und sich also a priori nicht geben lassen." $(\mathrm{A} 722 * \mathrm{~B} 750 *)$
}

Der Begriff der Ursache gehört zu der Klasse von Begriffen, die 'nicht aus der Erfahrung entlehnt' sind, sondern, transzendental gebraucht, diese Erfahrung allererst möglich machen, indem sie einen "Grundsatz der Synthesis möglicher empirischer Anschauungen" (A722 B750) formulieren. So bestimmt, ist der Begriff der Ursache Bestandteil eines transzendentalen, und d.h. nach Kant eines genuin philosophischen Satzes, der wie mathematische Sätze - die ihrerseits eine Synthesis von Mannigfaltigem ausdrücken (A720 B748) - synthetisch a priori gelten soll, aber nicht konstruierbar ist. In synthetischen Sätzen a priori beziehe ich mich auf die Eigenschaften des durch den Begriff bezeichneten Gegenstandes, die nicht im Begriff enthalten sind, doch zu ihm gehören. Diese Beziehung, so erläutert Kant in A718 B746, sei 
"nicht anders möglich, als daß ich meinen Gegenstand nach den Bedingungen, entweder der empirischen Anschauung, oder der reinen Anschauung bestimme. Das erstere würde nur einen empirischen Satz [...], der keine Allgemeinheit, noch weniger Notwendigkeit enthielte, abgeben [...] Das zweite Verfahren aber ist die mathematische [...] Konstruktion, vermittelst deren ich in der reinen Anschauung, ebenso wie in der empirischen, das Mannigfaltige, was zu dem Schema eines Triangels überhaupt, mithin zu seinem Begriffe gehört, hinzusetze, wodurch allerdings allgemeine synthetische Sätze konstruiert werden müssen."

Kant spricht hier von einer Dublette solcher synthetischen Erkenntnisse, die sich auf eine Mannigfaltigkeit von Anschauungen beziehen. Im Unterschied zu empirischen Sätzen gehen mathematische Sätze auf reine Anschauungen. Von transzendentalen Sätzen spricht Kant hier insofern, als empirische Sätze nur unter Voraussetzung der transzendentalen Synthesis empirischer Anschauungen möglich sind. Sofern empirische Sätze naturwissenschaftlich beschreibbar sind, sind sie auch nach den Bedingungen reiner Anschauung bestimmbar. Transzendentalen und mathematischen Sätzen ist gemeinsam, daß sie die Synthesis von Anschauungsmannigfaltigkeiten angeben. Die Differenz beider Erkenntnisarten zeigt sich daran, daß der transzendentale Satz eine Synthesis empirischer, der mathematische Satz eine Synthesis reiner Anschauungen formuliert. Der Rekurs auf die unterschiedlichen Anschauungstypen macht jedoch nicht den Grund der Differenz aus. Denn in dem zitierten Textstück führt Kant nur einen indirekten Vergleich beider Erkenntnisarten über die Entgegensetzung mathematischer und empirischer Sätze. Der Vergleich gerät dadurch zusätzlich in Schieflage, daß die Geltung mathematischer Sätze als Erkenntnis die Geltung transzendentaler Sätze voraussetzt und mathematische Sätze somit selber auf die Beschreibung empirischer Daten mögliche Anwendung finden müssen. Denn nur unter dieser Bedingung ist es sinnvoll zu behaupten, daß mathematischen Begriffen 'objektive Realität' zukomme:

"Es hat zwar den Anschein, als wenn die Möglichkeit eines Triangels aus seinem Begriffe an sich
selbst könne erkannt werden (von der Erfahrung ist er gewiß unabhängig); denn in der Tat können
wir ihm gänzlich a priori einen Gegenstand geben, d.i. ihn konstruieren. Weil dieses aber nur die
Form von einem Gegenstande ist, so würde er doch immer nur ein Produkt der Einbildung bleiben,
von dessen Gegenstand die Möglichkeit noch zweifelhaft bliebe, als wozu noch etwas mehr erfordert
wird, nämlich daß eine solche Figur unter lauter Bedingungen, auf denen alle Gegenstände der
Erfahrung beruhen, gedacht sei." (A223f. B271)

Dieses Zitat stützt erneut die These, daß Kants Darstellung mathematischer Begriffe nur unter Berücksichtigung der ihr im transzendentalen Begründungsprogramm zugedachten Funktion erschlossen werden kann. Dies bestätigen auch seine im Vergleich zum transzendentalphilosophischen Kontext durchweg negativen Bestimmungen mathematischer Inhalte: "alle mathematischen Begriffe sind für sich nicht Erkenntnisse" (B147); "in den mathematischen Aufgaben ist überhaupt von der Existenz gar nicht die Frage" (B747); "Mathematische Definitionen können niemals irren" (B759); dies bedeutet, daß sich für die Gegenstände der reinen Mathematik die Frage der empirischen Überprüfbarkeit der Wahrheit ihrer Aussagen gar nicht stellen kann. Zwar sind ihre Gegenstände durch "willkürliche Synthesis" definierbar, "aber ich kann nicht sagen, daß ich dadurch einen wahren Gegenstand definiert habe." (A729 B757)

Die Funktion der Mathematik ist vor dem Horizont der transzendentalen Theorie der Erfahrung überhaupt bislang nur negativ in bezug auf das Aufgabengebiet dieser Theorie charakterisiert worden. Durch die nun folgende Interpretation der MANW kann ihre 
Funktion positiv beschrieben werden. Das Beweisziel der $M A N W$, die Möglichkeit der Erkenntnis a priori bestimmter Naturdinge aufzuzeigen, fordert den Nachweis der Mathematisierbarkeit der physikalischen Leitbegriffe, genauer der materiellen Entitäten, die nach diesen Begriffen empirisch bestimmbar sind. Dabei zeichnet sich folgende Konfiguration von mathematischer und philosophischer Erkenntnisart ab: Während durch die metaphysische Rechtfertigung der Anwendung der Mathematik in der Naturwissenschaft die Begriffe und Lehrsätze "eigentlich der Transzendentalphilosophie" erst realisiert werden, ihr also "Sinn und Bedeutung" ${ }^{50}$ verschaffen, ist es gerade das Unterscheidungskriterium der 'Konstruktion der Begriffe', das Kant als das negative Merkmal der Definition der Transzendentalphilosophie in der $\mathrm{KrV}$ einführt. Diesen Zusammenhang werde ich in Kap.7 im Rahmen von Schellings Interpretation des Konstruktionstheorems entwickeln. 


\section{Der Begriff der Konstruktion in Kants Metaphysischen Anfangsgründen der Naturwissenschaft}

Kants Anliegen ist es, Newtons Mechanik auf die Basis einer dynamischen Naturphilosophie zu stellen, zu deren metaphysischen Grundannahme es gehört, der Materie wesentlich eigene Grundkräfte zuzuschreiben. Die metaphysisch-dynamische Erklärungsart soll sich der mathematisch-mechanischen Erklärungsart in der Begründung als überlegen erweisen. Im Anschluß an 1.4., Kap.2 und 4.3. zeige ich, wie Kant diese Priorität im Rückgriff auf transzendentallogische Theoreme plausibel machen will. Dabei gewinnt die Frage, wie sich die Konstruierbarkeit der Grundkräfte zu dieser Begründungsabsicht verhält, zentrale Bedeutung für die Bestimmung des Konstruktionsbegriffs in den $M A N W$. Die Untersuchung des Konstruktionsbegriffs setzt bei den wahrheitstheoretischen Aspekten der Theorie ein, die sich als 'Realisierung' der Begriffe und Lehrsätze der Transzendentalphilosophie versteht. Sie wird daher im Ausgang von den MAPhän in umgekehrter Reihenfolge der Hauptstücke die Anwendung des Konstruktionstheorems in den $M A N W$ zu beschreiben und zu erschließen versuchen.

\subsection{Die 'bloß metaphysische Erklärung' des Begriffs der Materie}

Den vier Hauptstücken der $M A N W$ ist jeweils eine "Erklärung" des Begriffs der Materie vorangestellt, die mit der gleichlautenden Formel beginnt: "Materie ist das Bewegliche [...]". ${ }^{1}$ In die Erklärungen geht damit als wesentlicher Bestandteil der Begriff der Bewegung als die "Grundbestimmung" $(22)^{2}$ der Materie ein. Zu dieser Grundbestimmung kommt in jedem der Hauptstücke "eine neue Bestimmung" hinzu, die dadurch gewonnen wird, daß der Begriff der Materie nach dem Leitfaden der Kategorientafel durch alle vier Funktionen der Kategorien "durchgeführt" wird.(22) Die Bestimmung der Materie als des Beweglichen ist daher nicht Resultat, sondern Voraussetzung der Durchführung. Die durch diese Operation systematisch aufgesuchten Merkmale der Materie sind diejenigen Prädikate des Objekts 'Materie', "die zu ihrer Natur gehören"(22). Nun heißt es, daß der Verstand diese Prädikate auf eben die Grundbestimmung der Materie "zurückführt".(22) Diese Reduktion bedeutet nicht die analytische Herleitung der Bestimmungen aus dem Begriff der Materie, sondern besagt, daß die Bestimmungen nicht ohne Bezug auf die Grundbestimmung prädizierbar sind.

Hervorhebung in Phor.Erkl.1 und Dyn.Erkl.1, ohne Hervorhebung Mech.Erkl.1 und Phän.Erkl.

2 Wegen der hohen Dichte der Textnachweise in Kap. 5 erfolgt die Seitenangabe der Zitate aus den MANW direkt im Text, und zwar nur mit Angabe der Seitenzahl nach WW V 7-135. Ich ziehe die Ausgabe WW der Ausgabe AA vor, weil sie unter textkritischen Gesichtspunkten in der Konstitution des Textes zuverlässiger und nach der formalen Gestaltung des Textes besser lesbar ist. 
Das Resultat der Durchführung des Begriffs der Materie in den ersten drei Hauptstücken ist die Bestimmung der Materie als des Beweglichen im Raume (Phor.Erkl.1), das einen Raum erfüllt (Dyn.Erkl.1) und als solches bewegende Kraft hat (Mech.Erk1.1). Diese sog. objektiven Bestimmungen kommen nicht bloß additiv und in beliebiger Reihenfolge zum Begriff der Materie hinzu. Ihre Zuordnung kann vielmehr als ein Bedingungsgefüge angesehen werden, nach dem die jeweils folgende Bestimmung nicht ohne Bezug auf die vorangehende Bestimmung und überhaupt nicht ohne Bezug auf die Grundbestimmung dem Begriff der Materie zugeschrieben wird. Darauf weist die Bemerkung Kants, daß die Dynamik die phoronomische, die Mechanik die dynamische Erklärung 'voraussetzen'.(47, 100)

Die Phänomenologie nimmt in besonderer Weise Bezug auf die ihr vorgeordneten Hauptstücke: Sie bestimmt den Begriff der Materie als des Beweglichen "bloß in Beziehung auf die Vorstellungsart, oder Modalität, mithin als Erscheinung äußerer Sinne" (22) 'in Ansehung' der Phoronomie, Dynamik und Mechanik. (124, 126, 127) Nach den in den MAPhän formulierten Kriterien kann entschieden werden, ob ein 'materielles Ding', dessen Bewegung wir wahrnehmen oder wahrzunehmen glauben, sich möglicherweise oder unmöglich, wirklich oder nur scheinbar oder sogar notwendigerweise bewegt. Die phänomenologische Erklärung fügt dem Begriff der Materie in diesem Sinne keine neue Bestimmung hinzu, sondern legt den Geltungsbereich der Aussagen fest, in welche die objektiven Bestimmungen jeweils eingehen. Phän.Erkl. lautet: "Materie ist das Bewegliche, sofern es, als ein solches, ein Gegenstand der Erfahrung sein kann."(122)

Vergleicht man Betrachtungsweise und Zielbestimmung der MAPhän, so bleibt zunächst offen, warum Materie als Gegenstand möglicher Erfahrung zum Thema gerade der Erscheinungslehre - so übersetzt Kant in $128^{*}$ - wird. Zu vermuten ist, daß, ausgehend von der Kennzeichnung der Bewegung als 'Erscheinung äußerer Sinne', die MAPhän die Kriterien angeben, nach denen Materie, grundlegend durch die Eigenschaft der Beweglichkeit bestimmt, als 'Gegenstand möglicher Erfahrung' aufgefaßt werden kann.

Kant wird in der Anmerkung zu Phän.Erkl. plakativ von der "Verwandlung [...] der Erscheinung in Erfahrung" (122f.) sprechen. Nach Auskunft der Vorrede betrachten die MAPhän die Bewegung materieller Dinge "bloß" in der Perspektive des vorstellenden Subjekts, "mithin als Erscheinung äußerer Sinne" (22). Diese Beschreibung legt den Vergleich mit der von Kant so genannten 'bloß metaphysischen Erklärung' des Begriffs der Materie nahe, die Kant in Anm.2 zu Phor.Erkl.1 folgendermaßen einführt:

\footnotetext{
"Wenn ich den Begriff der Materie nicht durch ein Prädikat, was ihr selbst als Objekt zukommt, sondern nur durch das Verhältnis zum Erkenntnisvermögen, in welchem mir die Vorstellung allererst gegeben werden kann, erklären soll, so ist Materie ein jeder Gegenstand äußerer Sinne, und dieses wäre die bloß metaphysische Erklärung derselben." (25)
}

Kant will zwei Arten von Erklärungen unterschieden wissen. Die phoronomische, dynamische und mechanische Erklärung sollen 'den Begriff der Materie durch ein Prädikat, was ihr selbst als Objekt zukommt, erklären'. Durch diese Erklärungen wird die Materie durch die Eigenschaft der Beweglichkeit jeweils in objektiver Beziehung bestimmt. Wird jedoch, wie in der metaphysischen Erklärung, der Begriff der Materie "nur durch das Verhältnis zum Erkenntnisvermögen, in welchem mir die Vorstellung [der Bewegung] 
allererst gegeben werden kann", erklärt, so sei Materie als "ein jeder Gegenstand äußerer Sinne" zu betrachten. Im Vergleich stimmen die metaphysische und phänomenologische Erklärung zwar in der subjektiven Betrachtungsweise überein, unterscheiden sich jedoch in der Bestimmung der Materie als 'Gegenstand äußerer Sinne' und als 'Gegenstand der Erfahrung'. Es bleibt zu fragen, in welchem Verhältnis die metaphysische und phänomenologische Erklärung einerseits und die objektiven Erklärungen andererseits stehen. Bei der Klärung dieser komplexen Beziehung gehe ich von der Frage aus, (a) welcher epistemische Status Kants weitreichender Behauptung zuzumessen ist, Materie sei ein jeder Gegenstand äußerer Sinne. Im zweiten Schritt (b) ist das Ergebnis dieser Prüfung mit den Zielsetzungen der MANW zu vergleichen. Schließlich (c) soll die Argumentationsweise, in der Kant die metaphysische Erklärung expliziert, auf ihre Beziehungen zu Phor.Erkl.1 und Phän.Erkl. hin untersucht werden.

(a) Man hat immer wieder betont, daß Kant den Ansprüchen, die an die Auszeichnung einzelner Argumentationsschritte als "Erklärung" oder "Beweis" zu stellen sind, in der Durchführung nicht gerecht wird. ${ }^{3}$ Tuschling weist gar darauf hin, daß Kant über die eigenen Gliederungsprinzipien in Verwirrung gerate und die Begriffe 'Bewegung' und 'Materie' bereits im Aufriß der Hauptstücke in der Vorrede als Subjekte der Darstellung der $M A N W$ durcheinanderlaufen. ${ }^{4}$ Diesen Befund sucht man sich dadurch verständlich zu machen, daß Kant mit der 'Nachahmung' der mathematischen Methode, die er nach eigenem Bekunden auch "nicht mit aller Strenge befolgt" (24) habe, eine Darstellungsform gewählt hat, die zur Umsetzung des Programms 'metaphysischer Anfangsgründe' ungeeignet war und zunächst auch ihn selbst über die Mängel seines 'Entwurfs' (24) im unklaren ließ. Nun hat Cramer am Beispiel der Phor.Erkl.1 zu zeigen versucht, daß Kant in den $M A N W$ einen eigenen Typ von Sätzen verwendet, die den epistemischen Status von 'nicht-reinen synthetischen Urteilen a priori' haben. ${ }^{5}$ Dieser Rekonstruktionsversuch geht allerdings von der Voraussetzung aus, daß auch Kant 'Erklärung' als eine Definition verstanden wissen wollte, deren Anspruch auf universelle Gültigkeit durch den Nachweis der logischen Äquivalenz des zu Erklärenden und Erklärenden gerechtfertigt wird. ${ }^{6}$ Der Versuch, diesen Nachweis für die Aussage, Materie sei ein jeder Gegenstand der äußeren Sinne, zu erbringen, führt Cramer zu Schlußfolgerungen, deren Ergebnis zunächst kurz vorzustellen ist. $^{7}$

Kants Kennzeichnung des Begriffs der Materie als eines 'abgesonderten (obzwar an sich empirischen)' Begriffs (16) legt die Deutung nahe, dieser Begriff sei ein 'aus der Erfahrung abstrahierter' Allgemeinbegriff. Solche empirischen Allgemeinbegriffe bilden wir aufgrund komparativer Verallgemeinerung eines Merkmals, das der uns verfügbaren Menge von Objekten gemeinsam ist und von den Merkmalen, in denen sich die Objekte voneinander unterscheiden, abstrahiert wird. ${ }^{8}$ Unter dieser Voraussetzung ist die Behauptung der

\footnotetext{
Böhme (1986) 174.

Tuschling (1971) $115 \mathrm{f}$.

Cramer (1985) hat diesen Typ von Urteilen, den Kants Transzendentalphilosophie zwar vorsieht, aber nicht ausführt, zuerst auf den Begriff gebracht und seinem Problemgehalt nach grundlegend analysiert.

Cramer (1985) 132.

Cramer (1985) 132-140, 140-152.

Cramer (1985) $107 f$.
} 
metaphysischen Erklärung, "daß wir mit keinem Gegenstand äußerer Sinne bekannt sein können, der nicht unter den Begriff 'Materie' fällt", nicht haltbar. Denn es ist nicht ausgeschlossen, daß wir von Gegenständen äußerer Sinne Erfahrung machen, die nicht materielle Dinge sind. ${ }^{9}$ Um diese Konsequenz zu vermeiden, darf der Begriff der Materie nicht als ein Prädikat verwendet werden, das dem Gegenstandsbereich, den es beschreibt, zukommt. ${ }^{10}$ Der Begriff darf kein 'normaler empirischer Allgemeinbegriff' sein, der einen besonderen (und darum erweiterbaren) Bereich von Gegenständen der äußeren Sinne bezeichnet. Er muß der Begriff von einer Gattung sein, unter den alle solchen empirischen Allgemeinbegriffe fallen und von dem gilt, "daß in ihm gar kein deskriptives Prädikat der Gegenstände äußerer Sinne vorgestellt wird."11 Cramer schlägt daher vor, die metaphysische Erklärung nur als eine "terminologische Festsetzung" zu betrachten, in der Kant "den Begriff 'Gegenstand der äußeren Sinne' terminologisch mit dem Begriff 'Materie' identifiziert und damit die durch beide Begriffe beschriebenen Bereiche der Erfahrung koextensiv setzt." 12

Zur Stützung seiner Interpretation verweist Cramer auf die Erläuterungen zur metaphysischen Erklärung in Anm.2 zu Phor.Erkl.1, die "so etwas wie eine Begründung für die These von der Koextensivität der Begriffe 'Materie' und 'Gegenstand äußerer Sinne'"13 enthalten:

"Der Raum aber wäre bloß die Form aller äußeren sinnlichen Anschauung [...]. Die Materie wäre, im Gegensatz der Form, das, was in der äußeren Anschauung ein Gegenstand der Empfindung ist, folglich das Eigentlichempirische der sinnlichen und äußeren Anschauung, weil es gar nicht a priori gegeben werden kann." (25f.)

Für die Bedeutung des Begriffs der Materie ergebe sich aus dieser Interpretation insofern eine Einschränkung, als der Begriff als Gattungsbegriff nur die 'Materie der Sinnlichkeit' bezeichnet und nicht etwas, das selbst unter diesen Bereich der Gegenstände äußerer Sinne und auch nicht des inneren Sinnes fällt. Damit sei ausgeschlossen, "daß der empirische Gegenstand des inneren Sinnes, das durch Synthesis der diesem Sinn gegebenen Empfindungen konstituierte empirische Ich, als ein materielles Objekt gekennzeichnet werden kann." ${ }^{14}$ Mit dieser Folgerung entspricht Cramer der Angabe Kants, daß die MANW nur die Grundsätze der 'Körperlehre', nicht auch die der 'Seelenlehre' behandeln können.(16)

Gewichtiger ist freilich die Folgerung, die Cramer aus der Begründung der Koextensivitätsthese der metaphysischen Erklärung für die Bestimmung der Materie als des Beweglichen im Raum in Phor.Erkl.1 zieht: Das Kriterium der Unterscheidung materieller und geometrischer Entitäten ist die Eigenschaft der Beweglichkeit. Kants Deutung der euklidischen Geometrie sieht denn auch die Bewegung geometrischer Entitäten (Drehung, Translation von Strecken) nicht vor. ${ }^{15}$ Im Gegensatz zu geometrischen

\footnotetext{
Ebd. 135.

Ebd. 139.

Ebd. 140.

Ebd. 137.

Ebd. 139.

14 Ebd. 138.

15 Ebd. 84-100, 360-363. Vgl. 5.7.
} 
Gegenständen, die in der reinen Anschauung des Raumes a priori konstruierbar sind, bezeichnet Materie einen Gegenstand der Empfindung. Die Exposition dessen, was Materie genannt wird, schließt damit notwendig den Bezug auf das 'Eigentlichempirische' ein, das 'gar nicht a priori' und nur in der sinnlichen und äußeren Anschauung gegeben werden kann. Unter dieser Einschränkung entspricht der Bereich materieller Gegenstände dem Bereich aller empirisch gebbaren Gegenstände der äußeren Sinne. ${ }^{16}$ Somit enthält die metaphysische Erklärung darüber hinaus, $d a \beta$ Materie ein Gegenstand äußerer Sinne ist, keine Informationen darüber, was Materie als Gegenstand äußerer Sinne ist.

"Sie gibt nur den Gegenstandsbereich an, auf den sich Metaphysische Anfangsgründe der Naturwissenschaft beziehen können sollen, ist aber eben deshalb keine Aussage, die selber als ein solcher Anfangsgrund, d.h. als eine Aussage verstanden werden kann, die von Materie etwas synthetisch und a priori prädiziert." 17

Mit Bezug auf Phor.Erkl.1 führt Cramer aus: Das Urteil, in dem der von Kant so genannte 'an sich empirische' Begriff der Materie grundlegend durch das Prädikat des Beweglichen bestimmt wird, trifft im Rekurs auf die metaphysische, von Cramer so genannten 'subjektiven' Erklärung des Begriffs der Materie eine Aussage über Gegenstände der äußeren Sinne. Da die MANW sich als ein System metaphysischer, d.h. nicht-empirischer und zugleich synthetischer Sätze a priori verstehen lassen (müssen), muß auch Phor.Erkl.1 als ein synthetisches Urteil a priori rekonstruierbar sein. Sofern die darin verbundenen Begriffe unbestreitbar einen empirischen Gehalt haben, muß es sich um ein 'nicht-reines synthetisches Urteil a priori' handeln.

"Die in Frage stehenden Begriffe müssen einerseits als nicht 'aus der Erfahrung abstrahierte' und in diesem präzisen Sinne apriorische Begriffe, andererseits als in spezifischer Weise auf empirische Anschauung bezogene und in diesem präzisen Sinne nicht-reine Begriffe interpretierbar sein." ${ }^{18}$

Was nach dieser Disposition unter dem genannten Urteilstyp genauer zu verstehen ist, wird in 5.6. anhand der Interpretation des in den MADyn eingeführten Theorems ursprünglicher Grundkräfte näher ausgeführt.

(b) Nimmt man einmal an, daß Kant bei der Formulierung der Erklärungen tatsächlich so etwas wie die semantische Gleichsetzung der Begriffe 'Materie' und 'Gegenstand äußerer Sinne' im Sinn hatte und unter dieser Voraussetzung Materie gemäß den objektiven Erklärungen bestimmen konnte, dann käme dies Überlegungen nahe, die z.B. Jammer als Antwort auf wahrheitstheoretische Probleme der Axiomatisierung der Mechanik vorgebracht hat. Wie in 2.1. dargestellt, geht es Jammer um die Frage, wie etwa der Grundbegriff der Masse in einem axiomatischen Formalismus so definiert werden kann, daß der Bedeutungsgehalt des Begriffs nach seiner formalisierten Definition mit dem Begriff, den wir aufgrund experimentell gewonnener Daten bilden, in Übereinstimmung zu bringen ist. Die Definitionen könnten nicht (wie etwa in der axiomatisierten Geometrie) der Theorie vorausgehen, sondern seien durch den Aufbau der Theorie unter Berücksichtigung der 'Korrelationen mit der Erfahrung', 'experimenteller Verfahrensweisen und Messungen'

$\begin{array}{ll}16 & \text { Ebd. } 139 . \\ 17 & \text { Ebd. } 140 . \\ 18 & \text { Ebd. } 154 .\end{array}$ 
sowie 'semantischer Regeln' zu gewinnen. ${ }^{19}$ In 2.1. habe ich bereits ausgeführt, inwiefern die Zielsetzungen der $M A N W$ diesen von Jammer verallgemeinerten Anforderungen an eine Metatheorie der Mechanik entsprechen. Kants metaphysische Theorie gibt die Bedingungen an, unter denen Erfahrung, verstanden als empirische Erkenntnis, erst möglich und formulierbar ist, und zwar so, daß der Wahrheitswert empirischer Erkenntnis entschieden werden kann. Es charakterisiert diese Form der Erkenntnis, daß sie den Bedingungen mathematisch-physikalischer Beschreibung genügt. Ohne Berücksichtigung dieser Kriterien könnten wir bei der Überprüfung physikalischer Hypothesen nicht gerechtfertigterweise auf 'Korrelationen mit der Erfahrung' verweisen. ${ }^{20}$

Was die von Cramer ins Spiel gebrachte semantische Gleichsetzung der Begriffe 'Materie' und 'Gegenstand äußerer Sinne' anlangt, so kommt diese doch ermäßigte Forderung an den Anspruch einer Definition wiederum methodologischen Vorstellungen nahe, die Kant in der $K r V$ im Hinblick auf den Status von Definitionen in philosophischen Theorien - und damit auch in den $M A N W$ - entwickelt: Definitionen am Anfang einer philosophischen Theorie dienen nur "zum bloßen Versuche" (A730 B758) und als "Annäherungen" zu korrekten (und korrigierbaren) Begriffsbestimmungen (A731* B759*). Dies gelte jedoch nicht für mathematische Definitionen, die, wie er sich ausdrückt, eben deshalb 'niemals irren können', weil sich für sie die Frage der Wahrheitsdifferenz gar nicht stellt: "Denn, weil der Begriff durch die Definition zuerst gegeben wird, so enthält er gerade nur das, was die Definition gerade durch ihn gedacht haben will." (A731 B759) Nach dieser Aussage enthält die Definition deshalb "nichts Unrichtiges" (A731 B759), weil der definierte Begriff "eine willkürliche Synthesis" ausdrückt, "welche a priori konstruiert werden kann". (A729 B757) Wie für "willkürlich gedachte" und darum erst definierbare Begriffe gilt jedoch auch für die Definition mathematischer Begriffe: "ich kann nicht sagen, daß ich dadurch einen wahren Gegenstand definiert habe." (A729 B757)

$\mathrm{Daß}$ es Kant in den MANW jedoch gerade um die Definition - er spricht im Falle philosophischer Definitionen genauer von Exposition oder Erklärung (A730 B758) 'wahrer Gegenstände', d.h. solcher Sachverhalte geht, über die ich in Form von Urteilen korrekte oder falsche Aussagen treffen kann, läßt sich an der Art, wie Kant die metaphysische Erklärung im einzelnen erläutert, verdeutlichen.

(c) Im Unterschied zur Phän.Erkl. "Materie ist das Bewegliche, sofern es, als ein solches, ein Gegenstand der Erfahrung sein kann."(122) spricht die 'bloß metaphysische Erklärung' weder von Materie als 'Beweglichem' noch als Gegenstand der 'Erfahrung', sondern von etwas, das aus der Perspektive des vorstellenden Subjekts 'ein jeder Gegenstand äußerer Sinne' ist. Nun erläutert Kant die Erklärung im Rekurs auf die Begriffe Form und Materie, die Kant zufolge "aller anderen Reflexion zum Grunde gelegt werden" (A266 B322).

19 Jammer (1981) 119-130.

20 Tatsächlich sind auch für Kant die quantitativen Aspekte seiner metaphysischen Grundlegung der Materietheorie entscheidend. Um zu zeigen, daß der Begriff der Masse, ungeachtet der Schätzung in extensiven Größen, durch die Einführung von Grundkräften dynamisch interpretiert werden kann, muß ihm an dem Nachweis gelegen sein, daß das Verhältnis, in dem Attraktions- und Repulsionskraft stehen, mathematisch beschrieben werden kann. - Vgl. 5.7. und Kants "kleine Vorerinnerung zum Behufe des Versuchs einer solchen vielleicht möglichen Konstruktion" (76-79). 
"Der Raum aber wäre bloß die Form aller äußeren sinnlichen Anschauung (ob eben dieselbe auch dem äußeren Objekt, das wir Materie nennen, an sich selbst zukomme, oder nur in der Beschaffenheit unseres Sinnes bleibe, davon ist hier gar nicht die Frage). Die Materie wäre, im Gegensatz der Form, das, was in der äußeren Anschauung ein Gegenstand der Empfindung ist, folglich das Eigentlichempirische der sinnlichen und äußeren Anschauung, weil es gar nicht a priori gegeben werden kann." (25f.)

Form und Materie werden im Text - der Anleitung zum Gebrauch der Reflexionsbegriffe in A266 B321 folgend - "in transzendentalem Verstande, da man von allem Unterschiede dessen, was gegeben wird, und der Art, wie es bestimmt wird, abstrahiert", angewendet. Deshalb wird ebensowenig wie im Wortlaut der Erklärung in diesem Teil ihrer Explikation die Eigenschaft der Beweglichkeit, durch die Materie grundlegend bestimmt ist, genannt.

Mit Hilfe dieser 'Reflexionsbegriffe' erschließt Kant die apriorischen und aposteriorischen Momente, nach denen wir den uns verfügbaren ('gegebenen') Begriff der Materie als des Beweglichen im Raum ins Verhältnis zum Erkenntnisvermögen (Verstand und Sinnlichkeit) setzen und damit auf seine transzendentalen Konstituenten hin untersuchen können. Diese "transzendentale Überlegung" (A261 B317) sei "vor allen objektiven Urteilen" zu dem Zweck anzustellen, das z.B. durch Form und Materie bezeichnete Verhältnis $^{21}$ gegebener Vorstellungen so $\mathrm{zu}$ bestimmen, daß klar werde, "in welcher Erkenntniskraft sie subjektiv zueinander gehören". (A261f. B317) Kant macht diese Überlegung zu einer "Pflicht" für jeden, der "a priori etwas über Dinge urteilen will." (A263 B319).

Ausgehend von den Reflexionsbestimmungen Form und Materie, setzt Kant, wie aus der Interpretation des Zitats (25f.) hervorgehen wird, die Momente der Anschauung, Empfindung, Wahrnehmung ins Verhältnis zu genau der Vorstellung der "Bewegung, die ein Gegenstand der Erfahrung ist" (26). Damit weist Kant nicht nur dem Wortlaut nach auf die Phän.Erkl. voraus; Anm.2 zu Phor.Erkl.1 ist in explikativen Teilen Vorgriff und Zitat von Phän.Ls.1 und Beweis (123f.), die denn auch "die Modalität der Bewegung in Ansehung der Phoronomie" (124) bestimmen. Daß Kant in der Explikation der wohlgemerkt 'bloß metaphysischen Erklärung' nach Maßgabe der MAPhän von den Reflexionsbegriffen der Materie und Form ausgeht, folgt auch aus dem - am Schema der Kategorientafel orientierten - "Plan" (B109f.) metaphysischer Theorien. Danach sind dem vierten Kategorientitel der Modalität die vierte Gruppe der Reflexionsbegriffe und das vierte Hauptstück der MANW systematisch zugeordnet. ${ }^{22}$

Kant beschreibt den Gehalt der metaphysischen Erklärung mit den Mitteln der transzendentalen Reflexion. Die metaphysische Erklärung ist insofern bei der Explikation der Phor.Erkl.1 vorauszusetzen, als sie den Gegenstandsbereich festlegt, auf den sich die objektiven Erklärungen des Begriffs der Materie als des Beweglichen beziehen: Materie ist "ein jeder Gegenstand äußerer Sinne". Nun wird der Gehalt der metaphysischen Erklärung eingangs mit Hilfe der Reflexionsbegriffe der Form und Materie entwickelt:

21 Die nach vier Gruppen geordneten Reflexionsbegriffe erläutert Kant im Kapitel "Von der Amphibolie der Reflexionsbegriffe": 1. Einerleiheit - Verschiedenheit, 2. Einstimmung - Widerstreit, 3. das Innere das Äußere, 4. Materie (das Bestimmbare) - Form (Bestimmung). (A260ff. B316ff.)

22 Den Zusammenhang der Begriffe 'Form' und 'Modalität' im Hinblick auf die Entstehung der Kantischen Urteilstheorie erörtert Schulthess (1981) 63-66. 
"Der Raum aber wäre bloß die Form aller äußeren sinnlichen Anschauung (ob eben dieselbe auch dem äußeren Objekt, das wir Materie nennen, an sich selbst zukomme, oder nur in der Beschaffenheit unseres Sinnes bleibe, davon ist hier gar nicht die Frage)." (25f.)

Kant ruft zunächst nur ein basales Theorem der Transzendentalphilosophie in Erinnerung, das er an anderer Stelle so formuliert:

"so geht die Form der Anschauung (als eine subjektive Beschaffenheit der Sinnlichkeit) vor aller Materie (den Empfindungen), mithin Raum und Zeit vor allen Erscheinungen und allen datis der Erfahrung vorher, und macht diese vielmehr allererst möglich." (A267 B323)

Indem 'die Form der Anschauung als eine subjektive Beschaffenheit der Sinnlichkeit' bestimmt wird, macht dieser Text der $K r V$ jedoch von einem Theorem Gebrauch, von dem in Anm.2 zu Phor.Erkl.1 "hier gar nicht die Frage" (26) sei. Die genannten Differenzpunkte liegen im Programm der MAPhor begründet, das eine Reihe von Abstraktionen fordert: Phoronomie, verstanden als "die reine Größenlehre (mathesis) der Bewegungen" (37, 45), betrachtet Materie unabhängig vom Begriff der Ausdehnung als einen Punkt (27, 37); Geschwindigkeit - das Moment der Erwägung von Bewegung, das auch den Zeitbegriff berïcksichtigt (37), - nimmt Kant "bloß in räumlicher Bedeutung" (31). Freilich handelt es sich hierbei auch nur um Abstraktionen von etwas, das, vollständig bestimmt, eben 'dem äußeren Objekt, das wir Materie nennen', entspricht. Die MAPhor exponieren den Begriff der Materie nur soweit, daß er - "zum Behuf der angewandten Mathematik" (34) - "a priori zur Anwendung auf äußere Erfahrung tauglich" (17) ist.

Es ist klar, daß Kant diese Zielvorgabe, die genaugenommen die Realisierung der "Begriffe und Lehrsätze [...] eigentlich der Transzendentalphilosophie" (24) sein soll, schon im Blick hat, wenn er mit der 'bloß metaphysischen Erklärung' Materie denn auch nur als 'einen jeden Gegenstand äußerer Sinne' erklärt. Formuliert Kant die Explikation der Erklärung eingangs noch im Irrealis ${ }^{23}$, so setzt er die zitierte Stelle bezeichnenderweise im Modus des Indikativ so fort:

"In aller Erfahrung muß etwas empfunden werden, und das ist das Reale der sinnlichen Anschauung, folglich muß auch der Raum, in welchem wir über die Bewegungen Erfahrung anstellen sollen, empfindbar [...] sein, und dieser, als der Inbegriff aller Gegenstände der Erfahrung und selbst ein Objekt derselben, heißt der empirische Raum. Dieser aber, als materiell, ist selbst beweglich. Ein beweglicher Raum aber, wenn seine Bewegung soll wahrgenommen werden können, setzt wiederum einen anderen erweitertern materiellen Raum voraus, in welchem er beweglich ist [...]. Also ist alle Bewegung, die ein Gegenstand der Erfahrung ist, bloß relativ". (26)

Kant expliziert den Begriff der Materie, anknüpfend an seine Bestimmung als des 'Beweglichen im Raum' in Phor.Erkl.1, nunmehr als 'Gegenstand der Erfahrung'. Damit impliziert die metaphysische Erklärung schon den Rekurs auf die Zielbestimmung der MAPhän: "Materie ist das Bewegliche, so fern es, als ein solches, ein Gegenstand der Erfahrung sein kann." (122)

Es ist daran $\mathrm{zu}$ erinnern, daß mit Ausnahme der metaphysischen Erklärung alle Erklärungen von der Bestimmung der Materie als des Beweglichen ausgehen, ergänzt jeweils unter dem spezifischen Aspekt des Hauptstücks. Phor.Erkl. spricht von Materie als

23 "Der Raum aber wäre bloß die Form [...]. Die Materie wäre, im Gegensatz der Form [...]". (25f.) 
dem Beweglichen im Raum, Dyn.Erkl. von Materie als dem Beweglichen, das einen Raum erfüllt. Im Unterschied zu diesen Bestimmungen thematisieren Mech.Erkl.1 und Phän.Erkl. das materielle Bewegliche "als ein solches" (100, 122). Damit ist für den Fall von Mech.Erkl.1 ausgesagt, daß Materie als das Bewegliche "durch seine Bewegung" bewegende Kraft haben soll, die als "die Kraft einer in Bewegung gesetzten Materie betrachtet" wird. (100) Darauf nimmt Phän.Erkl. Bezug, wenn es heißt, daß Materie als das Bewegliche "als ein solches, nämlich seiner Bewegung nach, bestimmt gedacht werden soll", und zwar - nun im Unterschied zu Mech.Erkl.1 - "zum Behuf einer möglichen Erfahrung". (122) Materie ist genau dann ein Gegenstand möglicher Erfahrung, wenn sie im Unterschied $\mathrm{zu}$ etwas bloß Beweglichem als das Bewegliche betrachtet wird, das bewegt oder in Bewegung ist. Nun ist Kant zufolge Bewegung nicht einfachhin erfahrbar, sondern Bewegung wird an etwas Beweglichem wahrgenommen, das, als ein solches, Gegenstand der Erfahrung ist. Im folgenden ist auf die Funktion der Phän.Erkl. für die objektiven Erklärungen einzugehen, um zu klären, was es heißt, Materie sei ein Gegenstand der Erfahrung.

\section{2. 'Bewegung als E rscheinung' und 'Bewegliches als Gegenstand der Erfahrung'}

Im Schlußsatz der Anmerkung zu Phän.Erkl. (122f.) beschreibt Kant das Verhältnis von Erscheinung und Erfahrung so, daß hierbei sinnvoll von der "Verwandlung [...] der Erscheinung in Erfahrung" die Rede sein könne. Wofür diese metaphorische Ausdrucksweise steht, läßt sich aus den ersten drei Sätzen der Anmerkung erschließen: 'Verwandlung' soll eine Verstandeshandlung beschreiben, die als Urteil interpretiert werden kann, dessen Bezugspunkte 'Bewegung als Erscheinung' und 'Bewegliches als Gegenstand der Erfahrung' sind. Die Struktur dieser Beziehung ist anhand der Interpretation der Anmerkung näher zu bestimmen. Die einschlägige Textpassage lautet:

\footnotetext{
"Bewegung ist, so wie alles, was durch Sinne vorgestellt wird, nur als Erscheinung gegeben. Damit ihre Vorstellung Erfahrung werde, dazu wird noch erfodert, daß etwas durch den Verstand gedacht werde, nämlich zu der Art, wie die Vorstellung dem Subjekte inhäriert, noch die Bestimmung eines Objekts durch dieselbe. Also wird das Bewegliche, als ein solches, ein Gegenstand der Erfahrung, wenn ein gewisses Objekt (hier also ein materielles Ding) in Ansehung des Prädikats der Bewegung als bestimmt gedacht wird."(122)
}

Die Anmerkung erörtert den Begriff der Bewegung von vornherein in der Weise des Vorliegens bzw. der Gegebenheit, d.h. in der Perspektive des vorstellenden Subjekts. Die Bestimmung der Bewegung als Erscheinung knüpft zwar an den Titel des Hauptstücks an, verlangt aber nach einem inhaltlichen Bezug zur Kennzeichnung des Beweglichen als eines Gegenstandes möglicher Erfahrung in der Erklärung.

Der erste Satz gibt Auskunft darüber, wodurch und wie Bewegung gegeben ist: nämlich durch die Sinne und als Erscheinung. Allerdings deutet die einschränkende Formulierung "nur als Erscheinung" schon auf ein Defizit in dieser Bestimmung. Das Kriterium der Vollständigkeit der Bestimmung des Bewegungsbegriffs wird aus der Bestimmung der Bewegung als eines Gegenstandes möglicher Erfahrung gezogen. 
Diesen Sachverhalt führt der zweite Satz aus, der Ziel und Mittel der Verstandeshandlung benennt: Damit die Vorstellung der Bewegung "Erfahrung werde", müsse "etwas durch den Verstand gedacht" werden. Der Ausdruck "etwas denken" wird näherhin als die "Bestimmung eines Objekts" durch die Vorstellung der Bewegung erläutert, wobei diese Bestimmung nicht ohne Bezug auf die "Art" zu leisten ist, "wie die Vorstellung dem Subjekte inhäriert". Mithin wird die Erscheinung in der Weise beschrieben, daß nicht sie, sondern durch sie ein Objekt bestimmt wird.

Den hier umschriebenen Sachverhalt bringt der schlußfolgernde dritte Satz in die Form eines Konditionalsatzes. Dabei nimmt der Trägersatz den Wortlaut der phänomenologischen Erklärung auf, das Antezedens gibt das Ergebnis des zweiten Satzes wieder. Im Urteil wird die Erscheinung in Erfahrung verwandelt, wenn "ein gewisses Objekt (hier also ein materielles Ding) in Ansehung des Prädikats der Bewegung als bestimmt gedacht wird."

Kant zufolge ist nicht einfachhin Bewegung erfahrbar, sondern Bewegung wird an etwas Beweglichem wahrgenommen, das, als ein solches, Gegenstand der Erfahrung ist. Als Gegenstand der Erfahrung wird ein materielles Ding durch etwas bezeichnet, das an ihm als Bewegung wahrgenommen wird. Von dem Beweglichen als Gegenstand spreche ich, wenn ich den Sachverhalt, daß ich die Bewegung von etwas wahrnehme, in der Form eines Urteils ausdrücke: 'x bewegt sich'. Folglich ist von einem Objekt erst dann zu sprechen, wenn es im Urteil durch das Prädikat der Bewegung bestimmt ist. Erst mit dieser Bestimmung ist es als etwas von seinem Erscheinen Unabhängiges und so im Unterschied zu 'Bewegung als Erscheinung' als 'Bewegliches als Gegenstand der Erfahrung' zu deuten.

Zwar hat Kant keine eigene Definition des Beweglichen gegeben. ${ }^{24}$ Der Ausdruck 'Wahrnehmung von Bewegung an etwas Beweglichem' kann jedoch mit Bezug auf Phor.Erk1.2, in der die "Bewegung eines Dinges" als "die Veränderung der äußeren Verhältnisse desselben zu einem gegebenen Raum" bestimmt wird (27), folgendermaßen präzisiert werden: Voraussetzung dafür, daß ich Bewegung wahrnehme, ist die Wahrnehmung von etwas Beweglichem als Beweglichem im Raum. Es ist die Wahrnehmung, daß etwas seine äußeren Verhältnisse $\mathrm{zu}$ einem gegebenen Raum verändern kann. ${ }^{25}$

Nun bezieht sich Wahrnehmung von etwas nicht auf Möglichkeiten, sondern auf wirkliche Gegebenheiten $^{26}$, die für den Fall, daß etwas Bewegliches wahrgenommen wird, auch "etwas Empirisches voraussetzen" (A41 B58). Dies ist auch Kants Argument für den Ausschluß des Begriffs der Bewegung aus dem Bereich der transzendentalen Ästhetik und Analytik. ${ }^{27}$ Obgleich Bewegung zur Sinnlichkeit gehört und beide Formen der Sinnlichkeit, Raum und Zeit, 'vereinigt', setzt die Wahrnehmung von Bewegung doch "die Wahrnehmung von etwas Beweglichem voraus." (A41 B58). Daher kann die Tatsache, "daß etwas beweglich sei, nicht a priori, sondern nur durch Erfahrung erkannt werden."

24 Darauf weist schon Cramer (1985) 83 hin.

25 Eine ausführliche Analyse dieses Sachverhalts gibt Cramer (1985) 83 und 103-107.

26 Ebd. 107.

27 Cramer (1985) 110ff. 
$\left(\mathrm{B} 155^{*}\right)^{28}$ Sofern also Bewegung "als Erscheinung gegeben" ist, enthält die Erscheinung über die Formen der Sinnlichkeit hinaus noch 'etwas Empirisches', das in Raum und Zeit, jedoch nicht schon mit Raum und Zeit gegeben ist. Dieses 'Eigentlichempirische' und 'Reale'(26) wird nun durch Empfindung vorgestellt, die, sofern sie in der Erscheinung zum Bewußtsein kommt, Wahrnehmung heißt.

\footnotetext{
"Wahrnehmung ist das empirische Bewußtsein, d.i. ein solches, in welchem zugleich Empfindung ist. Erscheinungen, als Gegenstände [!] der Wahrnehmung, sind nicht reine (bloß formale) Anschauungen, wie Raum und Zeit, (denn sie können an sich gar nicht wahrgenommen werden). Sie enthalten also über die Anschauung noch die Materien zu irgendeinem Objekte überhaupt (wodurch etwas Existierendes im Raume oder der Zeit vorgestellt wird), d.i. das Reale der Empfindung, also bloß subjektive Vorstellung, von der man sich nur bewußt werden kann, daß das Subjekt affiziert sei, und die man auf ein Objekt überhaupt bezieht, in sich." (B207f.)
}

In der Erfahrung liegt eine notwendige Verknüpfung der Wahrnehmungen (B218), die das Objekt in einer Bestimmtheit darstellt, durch die es als etwas von seinem Wahrgenommensein Unabhängiges vorgestellt wird. ${ }^{29}$ Damit ist eine Perspektive eröffnet, in der die Beziehung von 'Bewegung als Erscheinung' und 'Beweglichem als Gegenstand der Erfahrung' unter wahrheitstheoretischem Aspekt erläutert werden kann.

\subsection{Die MAPhän als 'physiologische Methodenlehre'}

In welcher Weise die Phänomenologie unter einem wahrheitstheoretischen Aspekt zu interpretieren ist, zeigt eine Bemerkung Kants, mit der er die Ambivalenz des Erscheinungsbegriffs zu klären sucht: In der Phänomenologie sei "nicht die Rede von Verwandlung des Scheins in Wahrheit, sondern der Erscheinung in Erfahrung"(122f.). Von dieser Ambivalenz spricht Kant in der Preisschrift, wo er Erscheinung, "im transzendentalen Sinn genommen, da man von Dingen sagt, sie sind Erscheinungen (phaenomena)" von der physischen Erscheinung (Apparenz) unterscheidet, "wenn ich sage, dieses Ding erscheint mir so oder so":

"Denn in der Sprache der Erfahrung sind diese Gegenstände der Sinne, weil ich sie nur mit andern Gegenständen der Sinne vergleichen kann, z.B. der Himmel mit allen seinen Sternen, ob er zwar bloß Erscheinung ist, wie Dinge an sich selbst gedacht, und wenn von diesem gesagt wird, er hat den Anschein von einem Gewölbe, so bedeutet hier der Schein das Subjektive in der Vorstellung eines Dinges, was eine Ursache sein kann, es in einem Urteil fälschlich für objektiv zu halten." ${ }^{30}$

Die ambivalente Bedeutung des Erscheinungsbegriffs resultiert daraus, daß er auf zwei Reflexionsebenen gebraucht werden kann. Auf der alltagssprachlichen Ebene bezeichnen physische Erscheinungen nur die Art, wie uns Dinge als Gegenstände gegeben sind. Als "Modifikationen" oder "innere Bestimmungen"(A197 B242) sind sie gültig im Hinblick "nur auf eine besondere Stellung oder Organisation dieses oder jenes Sinnes"(A45 B62). Den Gegensatz dazu bildet das von Prauss so genannte 'empirische Ding an $\operatorname{sich}^{131}$, das

28 Vgl. Cramer (1985) 110ff., 119ff.

29 Vgl. Cramer (1985) 20f.

30 F: AA XX 269 = WW III 600. Auf diese Stelle macht Prauss (1971) 19f. aufmerksam.

31 Prauss (1971) $15 \mathrm{ff}$. 
"unter allen verschiedenen Lagen zu den Sinnen, doch in der Anschauung so und nicht anders bestimmt ist."(A45 B63) Es gilt als das Objekt, das unabhängig von uns da und intersubjektiv erfahrbar ist. Kant nennt es das Phänomenon.

Die physische Erscheinung ist die Voraussetzung dafür, daß vom transzendentalen Standpunkt aus die empirischen Dinge an sich als Phänomene betrachtet werden. Diesen Sachverhalt bringt Kant auf die Formel: "Erscheinungen, sofern sie als Gegenstände nach der Einheit der Kategorien gedacht werden, heißen Phänomena." (A248f.) In der Formel werden die physischen Erscheinungen, sofern sie kategorial bestimmt sind, mit den empirischen Dingen an sich als Phänomenen identifiziert. In der transzendentalen Reflexion erweisen sich die Phänomena als zweifach vom Subjekt abhängig: als Erscheinungen unterliegen sie den Bedingungen der Sinnlichkeit, als kategorial bestimmte Erscheinungen den Bedingungen des Verstandes. ${ }^{32}$ "Der unbestimmte Gegenstand einer empirischen Anschauung heißt Erscheinung."(A20 B34) Als unbestimmter Gegenstand ist die empirische Erscheinung von ihrem Erscheinen (noch) nicht unabhängig zu denken.

Wenn die Grundsätze des reinen Verstandes allgemeine Sätze über die Anwendung der Kategorien auf Erscheinungen sind und als solche objektiv-synthetische Funktion haben, dann sind die "Postulate des empirischen Denkens überhaupt", die Kant als vierte Gruppe der Grundsätze aufführt, keine solchen Grundsätze. Sie überschreiten den Bereich der objektkonstituierenden Regeln in eine Metaebene, in der diese Funktionen selber thematisch werden. Die Postulate sind Sätze darüber, wie Urteilsobjekte 'sich zu Verstand, Urteilskraft und Vernunft in ihrer Anwendung auf Erfahrung verhalten'. Nach der Deutung von Krausser sind die Postulate genauer "metatheoretische Sätze darüber, welche Art von Geltungsanspruch für empirische, objekttheoretische Aussagen mit Bezug auf die darin angenommenen Objekte und Sachverhalte aufgestellt werden darf oder soll." ${ }^{33}$

Nach dieser Kennzeichnung erfüllen die MAPhän die Kriterien einer 'physiologischen Methodenlehre', deren Aufgabe Kant in der "Unterscheidung der Wahrheit und Hypothesen und die Grenzen der Zuverlässigkeit der letzteren" sieht. ${ }^{34}$ Die Vorstellung der Bewegung wird als die Erscheinung gegeben, die jeweils nach den phoronomischen, dynamischen und mechanischen Bestimmungen des Begriffs der Materie als des Beweglichen definiert ist. Wird durch diese Erscheinung ein Objekt bestimmt, das Gegenstand möglicher Erfahrung sein soll, ist Geltung und Wahrheitswert der Prädikation nach den in der MAPhän vorgegebenen Kriterien festzulegen.

Mit der Bestimmung unserer Vorstellungsart von Bewegung kommt dem Begriff der Materie in objektiver Beziehung keine neue Bestimmung hinzu. Das modale Prädikat

32 Prauss (1975) 21.

33 Krausser (1981) 137.

$34 \quad P$ §25: AA IV 307f. = WW III 174f.: "Zuletzt gehört auch zu den Erfahrungsurteilen die Erkenntnis der Übereinstimmung und Verknüpfung, nicht sowohl der Erscheinungen unter einander in der Erfahrung, als vielmehr ihr Verhältnis zur Erfahrung überhaupt, welches entweder ihre Übereinstimmung mit den formalen Bedingungen, welche die Verstand erkennt, oder Zusammenhang mit dem Materialen der Sinne und der Wahrnehmung, oder beiden in einen Begriff vereinigt, folglich Möglichkeit, Wirklichkeit und Notwendigkeit nach allgemeinen Naturgesetzen enthält, welches die physiologische Methodenlehre (Unterscheidung der Wahrheit und Hypothesen und die Grenzen der Zuverlässigkeit der letzteren) ausmachen würde." 
betrifft nicht den logischen Inhalt des empirischen Begriffs der Materie, der nach Quantität, Qualität und Relation kategorial erschöpfend darstellbar und als Objekt vollständig bestimmbar ist. (A74 B99f.) Die Modalität zeigt nur die Art an, wie der Begriff 'überhaupt mit der Erkenntniskraft verbunden wird' (A235 B287). ${ }^{35}$ Nun heißt es zum Programm der MAPhän:

"Nun ist aber Bewegung Veränderung der Relation im Raume. Es sind also hier immer zwei Correlata, deren einem in der Erscheinung erstlich eben so gut wie dem anderen die Veränderung beigelegt, und dasselbe entweder, oder das andere bewegt genannt werden kann, weil beides gleichgültig ist, oder zweitens, deren eines in der Erfahrung mit Ausschließung des anderen als bewegt gedacht werden muß, oder drittens, deren beide notwendig durch Vernunft als zugleich bewegt vorgestellt werden müssen. In der Erscheinung, die nichts als die Relation in der Bewegung (ihrer Veränderung nach) enthält, ist nichts von diesen Bestimmungen enthalten; wenn aber das Bewegliche als ein solches, nämlich seiner Bewegung nach, bestimmt gedacht werden soll, d.i. zum Behuf einer möglichen Erfahrung, ist es nötig, die Bedingungen anzuzeigen, unter welchen der Gegenstand (die Materie) auf eine oder andere Art durch das Prädikat der Bewegung bestimmt werden müsse."(122)

Die Anmerkung zu Phän.Erkl. nimmt verkürzt die Definition der Bewegung nach Phor.Erkl.2 als "Veränderung der Relation im Raume"(122) wieder auf. Als bewegt oder in Bewegung gilt etwas dann, wenn es seine äußeren Verhältnisse zu einem gegebenen Raum verändert. Etwas verändert sich dann, wenn in einem bestimmten Zeitintervall seine Zustände wechseln. Für den Fall der Bewegung gilt dann, daß etwas seine Lage im Raum relativ zu verschiedenen Örtern in einem bestimmten Zeitintervall verändert. Die Veränderung seiner Lage im Raum ist bestimmbar relativ zu dem Ort ${ }_{1}$, an dem es sich zu

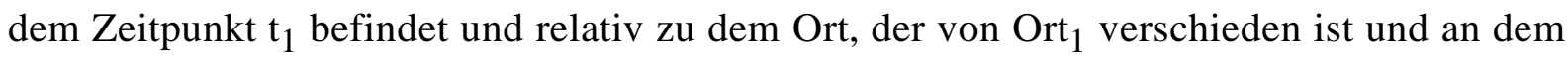
es sich zu dem Zeitpunkt $t_{2}$ befindet. "Es sind also hier immer zwei Correlata"(122), mit Bezug auf deren Relation etwas als bewegt bestimmt werden kann. Sofern Materie grundlegend durch die Eigenschaft der Bewegung bestimmt ist, wird Materie als Relat gedacht, das durch Veränderung seiner Relation zum umgebenden Raum erkannt wird.

Bewegung wird an etwas wahrgenommen, das während der Bewegung dasselbe bleibt; folglich setzt die Wahrnehmung von Bewegung etwas Beharrliches voraus, dessen Zustände wechseln. Nun ist Bewegung die Bestimmung einer Substanz und bezeichnet als deren Akzidenz eine 'besondere Art derselben zu existieren'. "Wenn man nun diesem Realen an der Substanz ein besonderes Dasein beigelegt, (z.E. der Bewegung, als einem Akzidenz der Materie,) so nennt man dieses Dasein die Inhärenz". (A186 B229f.) Betrachtet wird hier also das Verhältnis, wie Substanz und Akzidenz gesetzt sind. Inhärenz bezeichnet dabei die Art der Verknüpfung von Substanz und Akzidenz durch das vorstellende Subjekt. ${ }^{36}$

"Was wir auch nur an der Materie kennen, sind lauter Verhältnisse [...]. Freilich macht es stutzig, zu hören, daß ein Ding ganz und gar aus Verhältnissen bestehen solle, aber ein solches Ding ist auch [!]

35 "Wenn der Begriff eines Dinges schon ganz vollständig ist, so kann ich doch noch von diesem Gegenstande fragen, ob er bloß möglich, oder auch wirklich, oder, wenn er das letztere ist, ob er gar auch notwendig sei? Hierdurch werden keine Bestimmungen mehr im Objekte selbst gedacht, sondern es frägt sich nur, wie es sich (samt allen seinen Bestimmungen) zum Verstande und dessen empirischen Gebrauche, zur empirischen Urteilskraft, und zur Vernunft (in ihrer Anwendung auf Erfahrung) verhalte?" (A219 B266)

36 Vgl. Schulthess (1981) 63, 106f. 158-154, 208f. Das Realwesen der Sache wird durch die Art des Existierens bestimmt. 
bloße Erscheinung, und kann gar nicht durch reine Kategorien gedacht werden; es besteht selbst in dem bloßen Verhältnisse von Etwas überhaupt zu den Sinnen." (A285 B341)

Soll ein objektives Urteil gefällt werden, ist zunächst zu klären, wie die Vorstellung der Bewegung im Subjekt erscheint' (gradlinig, kreisförmig), um danach zu entscheiden, welchem Korrelat die Eigenschaft der Bewegung beigelegt werden darf und muß. Während in der formalen Logik beim disjunktiven Urteil "lediglich von dem Verhältnis des Erkenntnisses zum Objekte" die Rede sei, komme es in der Erscheinungslehre auf das Verhältnis zum Subjekt an, "um darnach das Verhältnis der Objekte zu bestimmen".(128*)

Zunächst betont Kant, daß "in der Erscheinung, die nichts als die Relation in der Bewegung (ihrer Veränderung nach) enthält", nichts von den Bestimmungen enthalten sei, durch die von einem Gegenstand die Eigenschaft, bewegt zu sein, ausgesagt wird. Im Urteilsakt gilt für den phoronomischen Fall der Bewegung:

"Es sind also hier immer zwei Correlata, deren einem in der Erscheinung [...] eben so gut wie dem anderen die Veränderung beigelegt, und dasselbe entweder, oder das andere bewegt genannt werden kann, weil beides gleichgültig ist". (122)

Im Falle der gradlinigen Bewegung ist ohne zusätzliche Informationen (z.B. Gegendruck bei einer beschleunigten Bewegung) nicht entscheidbar, welcher Gegenstand sich bewegt.

"Ob ein Körper im relativen Raume bewegt, dieser aber ruhig genannt werde, oder umgekehrt, dieser in entgegengesetzter Richtung gleich geschwinde bewegt, dagegen jener ruhig genannt werden solle, ist kein Streit über das, was dem Gegenstande, sondern nur seinem Verhältnisse zum Subjekt, mithin der Erscheinung und nicht der Erfahrung, zukommt."(123)

Im phoronomischen Fall erlaubt die Erscheinung "nicht die Bestimmung nach einem disjunktiven, sondern bloß die Wahl nach einem alternativen Urteile", wobei die Prädikationen, ob etwas beweglich ist oder ruht, hierbei 'objektiv zwar gleichgeltend, subjektiv aber einander entgegengesetzt sind, ohne Ausschließung des Gegenteils vom Objekt'.(124) Das Urteil gilt "nur in subjektiver Beziehung".(128*) Durch "den Begriff der Bewegung, als Gegenstandes der Erfahrung, ist es an sich unbestimmt [!], mithin gleichgeltend, ob ein Körper im relativen Raume, oder dieser in Ansehung jenes als bewegt vorgestellt werde."(124)

Im phoronomischen Fall einer gradlinigen Bewegung von etwas kann das Bewußtsein, das der Betrachter von der Erscheinung hat, in der von Prauss (1975) vorgeschlagenen Form eines Wahrnehmungsurteils so ausgedrückt werden: "Es scheint, daß x sich bewegt und y ruht." - "Ich habe Erscheinungen, welche die Deutung nahelegen, daß x sich bewegt und y ruht." Bei der Erscheinung der gradlinigen Bewegung können 'Beharrliches' und 'Veränderliches' nicht eindeutig zugeordnet werden. Sofern das Urteil bloß subjektiv gültig ist, könnte sich die Zuweisung der Prädikate auch umgekehrt verhalten. Soll das Objekt für alle Erscheinungen gültig bestimmt sein, ist der durch das Prädikat bezeichnete Sachverhalt 'bloß möglich'. Auf die auf Wahrnehmungs- und Erfahrungsurteile bezogene Differenz von subjektiver und objektiver Gültigkeit geht die "Unterscheidung der Wahrheit und Hypothesen" - so wird in P $\$ 25$ das Aufgabengebiet der MAPhän als "Methodenlehre" beschrieben - zurück. Der in den MAPhän thematisierte Begriff der Bewegung als Erscheinung ist bereits als theoretisch komplexes Gebilde zu interpretieren. Soweit die vorgelegte Interpretation zeigt, sind es gerade wahrheitstheoretische Aspekte der 
transzendentalen Theorie der Erfahrung, die sich an der phänomenologischen Betrachtung der Materie entwickeln und die metatheoretische Stellung der MAPhän in der internen Systematik der vier Hauptstücke der MANW erkennen lassen.

Die Analyse des Bewegungsbegriffs in den MAPhän soll im Rahmen dieser Untersuchungen nur so weit geführt werden, daß ihre Bedeutung für die Erschließung des Konstruktionsproblems in den MANW - der Begriff der Konstruktion tritt in den MAPhän gar nicht auf - deutlich wird. Denn es wird gerade die mathematische Konstruierbarkeit der physikalischen Leitbegriffe sein, die dafür einsteht, daß die durch diese Begriffe bezeichneten empirischen Sachverhalte die objektive Realität gerade derjenigen transzendentalen Begriffe und Lehrsätze ausweisen, unter deren Voraussetzung die Bestimmung des Begriffs der Materie in den objektiven Erklärungen der MAPhor, MADyn und MAMech erfolgt. Dabei werden in den Erklärungen jene Leitbegriffe "der Bewegung, der Erfüllung des Raums, der Trägheit, u.s.w."(17) im Hinblick auf ihre metaphysischen, d.h. a priori gegebenen Gehalte nach den Kriterien der kritischen Metaphysik so eingeführt, daß sie den durch sie naturwissenschaftlich beschreibbaren Begriff der Materie tatsächlich "zur Anwendung auf äußere Erfahrung tauglich machen" (17).

\subsection{Die metaphysisch-dynamische Erklärungsart des Begriffs der Materie}

Kants Anliegen ist es, Newtons Mechanik auf die Basis einer dynamischen Naturphilosophie zu stellen, zu deren metaphysischen Grundannahme es gehört, der Materie wesentlich eigene Grundkräfte zuzuschreiben. In der "Vorrede" der MANW vertritt Kant die These, daß 'alle Naturphilosophen, welche in ihrem Geschäfte mathematisch verfahren wollten, sich jederzeit (obschon sich selbst unbewußt) metaphysischer Prinzipien bedient hätten.'(17) Kant geht es nicht nur darum, diesen 'unbewußten' und daher unkritischen Gebrauch metaphysischer Prinzipien auf erkenntnistheoretische Mängel hin zu untersuchen. Er versucht darüber hinaus aus der Art, wie sich der Rückgriff auf metaphysische Prinzipien als notwendig ausweisen läßt, Argumente für den Vorrang für das von ihm gegen die Atomisten verfochtene Materiemodell zu gewinnen.

\footnotetext{
"So konnten also jene mathematische Physiker metaphysischer Prinzipien gar nicht entbehren, und unter diesen auch nicht solcher, welche den Begriff ihres eigentlichen Gegenstandes, nämlich der Materie, a priori zur Anwendung auf äußere Erfahrung tauglich machen, als des Begriffs der Bewegung, der Erfüllung des Raums, der Trägheit, u.s.w." (17)
}

Der Begriff der Materie ist dann 'a priori zur Anwendung auf äußere Erfahrung tauglich', wenn er in objektiver Beziehung bestimmt ist. Die objektive Bestimmung folgt aus der 'Durchführung' des Begriffs in den ersten drei Hauptstücken der $M A N W$, nämlich aus der Bestimmung der Materie als des Beweglichen im Raume (Phor.Erkl.1), das einen Raum erfüllt (Dyn.Erkl.1) und als solches bewegende Kraft hat (Mech.Erkl.1). Die Reihenfolge dieser Bestimmungen ist Ausdruck eines Bedingungsgefüges, nach dem die Dynamik die phoronomische Erklärung, die Mechanik wiederum die dynamische Erklärung 'voraussetzen'(47, 100). Diese Zuordnung der Hauptstücke gibt Aufschluß über die Funktionen, die die phoronomische und dynamische Erklärung des Begriffs der Materie im 
Begründungsgang der MANW haben: Zwar wird der Begriff der Materie im Kontext der Prinzipien dessen, was zum Dasein eines Dinges gehört, entwickelt, jedoch in Begriffen, die in der objektkonstituierenden Funktion quantifizierbar sind. Die Erklärung der Bewegung als Größe in den MAPhor soll daher 'zum Behuf der angewandten Mathematik' aufzeigen, wie 'die Regeln der Verknüpfung der Bewegungen durch physische Ursachen, d.h. Kräfte, rein mathematisch' beschrieben werden kann.(34)

Diese Kräfte, deren Wirkweise in den MAMech unter dem Stichwort 'Mitteilung der Bewegung' abgehandelt wird, sind von den in den MADyn eingeführten Grundkräften der Repulsion und Attraktion zu unterscheiden. Die Wirkweise solcher Kräfte erfordert Kant zufolge nämlich die Annahme von Grundkräften, da erst deren Konflikt und gegenseitige Einschränkung so etwas wie 'Raumerfüllung' (das, was die Atomisten als 'das Solide' schon zu den wesentlichen Eigenschaften der Materie zählen) uns begreifen lassen. Wenn der Mathematiker nun das Solide "als ein erstes Datum der Konstruktion des Begriffs der Materie, welches sich selbst nicht weiter konstruieren lasse", annehme (49), dann benutze er eine ontologische Hypothese, die im Unterschied zur metaphysisch-dynamischen Erklärungsart "die Richtigkeit der unserer Vernunfterkenntnis vergönneten Elemente der Konstruktion" (75) nicht gewährleisten könne. Die folgenden Untersuchungen sollen zeigen, wie Kant den von ihm behaupteten Vorrang der metaphysisch-dynamischen Erklärungsart begründet und welche Funktion der Konstruktionsbegriff bei diesem Begründungsversuch erfüllt. Aus den Analysen zu Absicht, Durchführung und Grenzen dieser Erklärungsart geht hervor, daß Kant in den $M A N W$ keinen anderen Begriff als den der mathematischen Konstruktion anwendet.

Die Priorität der dynamischen Erklärungsart in der Naturwissenschaft verdeutlicht Kant "an der vornehmsten aller ihrer Aufgaben, nämlich der Erklärung einer ins Unendliche möglichen spezifischen Verschiedenheit der Materien"(95), d.h. der Erklärung der Möglichkeit der spezifischen Dichten von Materien. Zur Lösung des Problems verfüge man über "nur zwei Wege"(95): Die Atomistik erkläre die unterschiedlichen Dichten "durch Verbindung des Absolutvollen mit dem Absolutleeren" (96) und bedürfe also der Annahme leerer Räume zwischen den Atomen. Diese Konzeption habe den Vorteil leichterer Anwendung der Mathematik, da sich die Möglichkeit der Konfigurationen und der leeren Zwischenräume 'mit mathematischer Evidenz' dartun lasse.(85) Doch werde dieser Vorteil um den Preis unhaltbarer Annahmen erkauft. Die mathematischmechanische Erklärungsart postuliere nämlich den 'leeren Begriff' der absoluten Undurchdringlichkeit der primitiven Materie, die absolute Gleichartigkeit des Stoffes sowie die absolute Unüberwindlichkeit des Zusammenhangs der Materie in den Grundkörperchen, die bloß durch die spezifische Gestalt voneinander unterschieden seien. Zudem seien alle der Materie eigenen Kräfte aufzugeben.(85, 96f.)

Diese Annahmen geißelt Kant als "Schlagbaum für die herrschende Vernunft", die so "auf dem Polster dunkler Qualitäten zur Ruhe gebracht werde"(95). So versucht Kant "die erste und vornehmste Beglaubigung" des atomistischen Systems dadurch zu erschüttern, daß er eine Möglichkeit aufzeigt, "sich einen spezifischen Unterschied der Dichtigkeit der Materien ohne Beimischung leerer Räume zu denken"(97). Er schlägt den der Atomistik 'entgegengesetzten' dynamischen Weg ein, "durch die bloße Verschiedenheit in der 
Verbindung der ursprünglichen Kräfte der Zurückstoßung und Anziehung alle Verschiedenheiten der Materien zu erklären." (96)

Die Voraussetzung dafür sieht Kant in einem 'transzendentalen Beweis' (A173f. B215), in dem er die Unmöglichkeit der Erfahrung leerer Räume aufweist und (ohne bereits auf das Konfliktmodell zweier Grundkräfte zu rekurrieren) einen Weg vorzeichnet, der die prinzipielle Vereinbarkeit der dynamischen Erklärungsart mit der kritischen Theorie des Erkenntnisvermögens aufweisen soll. Diesen Beweis führt Kant im 'Antizipationen der Wahrnehmung' genannten zweiten Grundsatzkapitel, das "die zweite Anwendung der Mathematik [...] auf Naturwissenschaft" begründen soll. ${ }^{37}$

\footnotetext{
"Das erste jener physiologischen Grundsätze subsumiert alle Erscheinungen, als Anschauungen im Raum und Zeit, unter den Begriff der Größe, und ist sofern ein Prinzip der Anwendung der Mathematik auf Erfahrung. Das zweite subsumiert das eigentlich Empirische, nämlich die Empfindung, die das Reale der Anschauungen bezeichnet, nicht geradezu unter den Begriff der Größe, weil Empfindung keine Anschauung ist, die Raum oder Zeit enthielte, ob sich gleich den ihr korrespondierenden Gegenstand in beide setzt; allein es ist zwischen Realität (Empfindungsvorstellung) und der Null, d.i. dem gänzlich Leeren der Anschauung in der Zeit, doch ein Unterschied, der eine Größe hat, da nämlich zwischen [...] jedem Grade der Erfüllung des Raumes und dem völlig leeren Raume, immer noch kleinere Grade gedacht werden können [...]; daher keine Wahrnehmung möglich ist, welche einen absoluten Mangel bewiese, [...] weswegen der Verstand so gar Empfindungen, welche die eigentliche Qualität der empirischen Vorstellungen (Erscheinungen) ausmachen, antizipieren kann, vermittelst des Grundsatzes, daß sie alle insgesamt, mithin das Reale aller Erscheinung Grade habe, welches die zweite Anwendung der Mathematik (mathesis intensorum) auf Naturwissenschaft ist." ${ }^{38}$
}

Der Grundsatz setzt voraus, daß sich etwas findet, "was sich an jeder Empfindung, als Empfindung überhaupt, (ohne daß eine besondere gegeben sein mag,) a priori erkennen läßt". (A167 B209) So soll der Grundsatz die graduelle Bestimmtheit des Realen oder der Qualität der Erscheinungen ausweisen, die einer Empfindung an einem Gegenstand korrespondiert. Antizipierbar ist nicht eine Mannigfaltigkeit von Empfindungen, die graduelle Unterschiede ihrer intensiven Größe aufweisen. Antizipierbar ist allein die intensive Quantität des Realen; von Größen überhaupt läßt sich nur ihre Kontinuität a priori aussagen.(A176 B218) ${ }^{39}$

Aufgrund der Beobachtung, daß die Quantität der Materie (Masse) unterschiedlicher Art bei gleichem Volumen veränderlich ist, folgern die Atomisten: "dieses Volumen (extensive Größe der Erscheinung) müsse in allen Materien, obzwar in verschiedenem Maße, leer sein." Diese Ansicht baut nach Kant auf der nicht ausgewiesenen metaphysischen Annahme, daß das Reale im Raum "allerwärts einerlei" sei und der spezifische Unterschied der Materien also auf das Volumen zurückgeführt werden müsse. Kant kritisiert, "daß man fälschlich das Reale der Erscheinung dem Grade nach als gleich, und nur der Aggregation und deren extensiven Größe nach als verschieden annehme"(A175 B216) und somit den spezifischen Unterschied der Materien vom Volumen abhängig macht.

37 P $\$ 24:$ AA IV $307=$ WW III 174.

38 P $\$ 24:$ AA IV 306f. = WW III 173f. Nach der Formulierung der 2. Aufl. der $K r V$ lauten der erste und zweite Grundsatz des reinen Verstandes "Axiome der Anschauung [...]: Alle Anschauungen sind extensive Größen." (B202) "Antizipationen der Wahrnehmung [...]: In allen Erscheinungen hat das Reale, was ein Gegenstand der Empfindung ist, intensive Größe, d.i. einen Grad." (B207)

39 Vgl. Cramer (1985) 205, $210 f$. 
Kants Alternative ist in der $K r V$ nur in vagen Strichen angedeutet. Danach soll aus der graduellen Bestimmtheit des Realen der Erscheinungen, gemessen am Moment des Widerstands oder Wiegens(A174 B216), die Verschiedenheit der Dichtigkeit der Materien volumenunabhängig hergeleitet werden. Kant weist jedoch darauf hin, daß sein Vorschlag bloß hypothetische Geltung hat: "Meine Absicht ist hier keineswegs, zu behaupten: daß dies wirklich mit der Verschiedenheit der Materien, ihrer spezifischen Schwere nach, so bewandt sei".(A174f. B216) Vielmehr wolle er bloß die Möglichkeit einer alternativen Erklärungsart, die mit den Theoremen der kritischen Theorie des Erkenntnisvermögens kompatibel sei, aufzeigen. Damit habe diese Erklärungsart, wie er in den MANW hervorhebt, den Vorzug, "die Naturphilosophie, so weit als es immer möglich ist, auf die Erforschung der dynamischen Erklärungsgründe zu leiten, weil diese allein [...] wahren Vernunftzusammenhang der Erklärungen, hoffen lassen." (98f.)

\subsection{Die dynamische Interpretation des Massenbegriffs}

Obwohl Kant das Konfliktmodell zweier Grundkräfte in der $K r V$ noch nicht ausdrücklich erwähnt, deutet der Hinweis, die Verschiedenheit der Materien von ihrer spezifischen Schwere her abzuleiten, auf den in den $M A N W$ unternommenen Versuch, die Grundbegriffe der Newtonschen Mechanik dynamisch zu interpretieren. Zur Zeit Newtons wird der Begriff der spezifischen Schwere synonym mit dem der Dichte gebraucht. ${ }^{40}$ In den $M A N W$ führt Kant den spezifischen Unterschied der Dichtigkeiten auf die unterschiedliche Erfüllung des Raumes durch repulsive Kraft zurück. Der Grad der Raumerfüllung resultiert zwar aus der Verbindung von Repulsions- und Attraktionskraft, doch während die Attraktion sich proportional zur Quantität der Materie verhält, kann die Repulsion gegen die Masse invariant und im Verhältnis zur Attraktion als "ursprünglich verschieden"(98) gelten. $^{41}$

Die Kräfte werden jeweils durch einen 'Widerstand' als eine Eigenschaft der Materie angezeigt, "die sich als Ursache auf eine Wirkung bezieht, nämlich das Vermögen, einer Bewegung innerhalb eines gewissen Raumes $\mathrm{zu}$ widerstehen"(47). Auf den Zusammenhang von Kraft und Kausalität deutet Kant bei seinen Erläuterungen zur graduellen Bestimmtheit der Realität in der Erscheinung: "Wenn man diese Realität als Ursache (es sei der Empfindung oder anderer Realität in der Erscheinung, z.B. einer Veränderung,) betrachtet; so nennt man den Grad der Realität als Ursache, ein Moment, z.B. das Moment der Schwere". (A168f. B210) Damit führt Kant an dieser Stelle schon ein Theorem des dritten Grundsatzkapitels ein, worauf er auch hinweist: "Dieses berühre ich

40 Jammer (1981) 70.

41 Materie erfüllt den Raum "durch repulsive Kraft, die ihren Grad hat, der in verschiedenen Materien verschieden sein kann, und, da er für sich nichts mit der Anziehungskraft, welche der Quantität der Materie gemäß ist, gemein hat, sie bei einerlei Anziehungskraft in verschiedenen Materien dem Grade nach als ursprünglich verschieden sein könne, folglich auch der Grad der Ausdehnung dieser Materien bei derselben Quantität der Materie und umgekehrt die Quantität der Materie unter demselben Volumen, d.i. die Dichtigkeit derselben ursprünglich gar große spezifische Verschiedenheiten zulasse."(97f.) 
aber hier nur beiläufig, denn mit der Kausalität habe ich für jetzt noch nicht zu tun."(A168f. B210)

Diese Bemerkung ist für die Aufklärung der internen Struktur der $M A N W$ von Bedeutung: Das Verhältnis von Substanz (Masse) und Qualität (Schwere) wird durch den Begriff der Kraft beschrieben. ${ }^{42}$ Sofern der Begriff der Materie als das Bewegliche erklärt wird, das den Raum erfüllt und als solches bewegende Kraft hat, entspricht die Kennzeichnung der Kraft als 'Kausalität einer Ursache' systematisch dem Fortgang vom zweiten zum dritten Kapitel der Grundsätze des reinen Verstandes bzw. von der Dynamik zur Mechanik.

Aus dieser ersten Problemskizze physikalischer 'Momente' und ihrer metaphysischen Interpretation ergeben sich zwei Fragenkomplexe: Zunächst ist zu klären, in welchem Verhältnis die Begriffe von Dichte und Schwere, Masse und Quantität der Materie sowie Trägheitskraft stehen. In 5.6. versuche ich aufzuzeigen, welcher Zusammenhang zwischen Kants Auffassung der Dichte als Raumerfüllung und seines Theorems zweier konfligierender Grundkräfte, deren Auszeichnung als Kausalität einer Ursache unseren Begriff der Raumerfüllung erst verständlich machen soll, besteht.

Newton hat den Begriff der Dichte bei der Systematisierung des Massenbegriffs schon in die Def.1 der Principia Mathematica aufgenommen: Masse ist das Produkt aus Dichte und Volumen. Die Quantität der Materie ist das Maß für dieselbe, aus deren Dichte und Größe gemeinsam gebildet. Nun hat Newton den Begriff der Dichte in der 1. Ausgabe seines Werkes nicht eigens bestimmt, und die Definition der Masse wäre dann zirkulär, wenn Dichte nur als Masse pro Volumeneinheit definiert werden kann. ${ }^{43}$

Dagegen versucht Jammer zu zeigen, "daß für Newton der Begriff der Dichte primär war und vor den Begriff der Masse gestellt werden muß"44, und zwar in einer Weise, die Dichte schon in Verbindung mit Kräften bringt. ${ }^{45}$ Mit Bezug auf Def.3, in der Newton die Trägheitskraft bestimmt, führt Jammer aus, daß "ein intensiver (durch innere Kraft wirkender) Faktor im Sinne von Volumen-Unabhängigkeit" den Unterschied der Quantität der Materie in Körpern von gleichem Volumen bedinge. "Und wegen der universalen Gültigkeit der Eigenschaft der Trägheit muß dieser Faktor auch für die kleinsten denkbaren Partikel gültig sein. Dieser Faktor ist in einem rein intensiven, qualitativen Sinn identisch mit 'Dichte' bei Newton". ${ }^{46}$

Für Newton war die Trägheitskraft schlicht eine Kraft der Inaktivität, deren Wirkung sowohl als Widerstand (resistentia) wie als Impuls (impetus) betrachtet werden kann. Leibniz hinterfragte diese Trägheitskraft: Die Masse, die fähig ist zu widerstehen, setzt selbst eine Kraftquelle voraus. Masse wird als Träger und Verteiler von Aktivität und Energie konzipiert. ${ }^{47}$ Anders als für Descartes, der den Körper bzw. die Materie wesentlich

42 A204 B249: "Diese Kausalität [der Ursache] führt auf den Begriff der Handlung, diese auf den Begriff der Kraft, und dadurch auf den Begriff der Substanz." - Daß Kant hierbei nur eine Begriffskette der Wolffschen Ontologie zitiert, belegt Cramer (1985) 246.

43 Jammer (1981) 72.

44 Ebd. 69.

45 Ebd. 71ff.

46 Ebd. 75.

47 Ebd. 85. 
nach Ausdehnung in Länge, Breite und Tiefe bestimmt sieht, läßt sich für Leibniz die Realität des Ausgedehnten nicht bloß durch seine mathematische Bestimmung erweisen. Der Ausdehnung, die keine primitive Bestimmung der Materie mehr ist, geht Aktion und Bewegung voran. Die Realität der ausgedehnten Materie beruht auf der Annahme von etwas, das die Konstitution der körperlichen Welt wie die Regeln der Bewegung hinreichend erklärbar macht. Leibniz führt dies als das 'Prinzip des Tuns und Leidens' unter der Bezeichnung to dynamikon ein. ${ }^{48}$

Leibniz' dynamische Interpretation des Massenbegriffs bereitet die Überwindung des Begriffs der Trägheitskraft durch Kant in den $M A N W$ vor. Jammer hält Kants Prüfung des ontologischen und methodologischen Status der Trägheitskraft für einen "wichtigeren Beitrag" zur physikalischen Grundlagenforschung: "Kants Ausschaltung der metaphysischen vis insita oder vis inertia legte den Weg für eine mehr positivistische Auffassung des Massenbegriffs frei." ${ }^{49}$ Noch in der Monadologia Physica (1756) führt Kant die Trägheit auf eine der Materie innewohnende Kraft zurück. Jedes Element eines Körpers hat eine spezifisch verschiedene Trägheitskraft (Prop.XI), die eine besondere Art der bewegenden Kraft ist (Coroll.I) ${ }^{50}$ Die Größe dieser Kraft setzt Kant mit der Masse der Körper gleich (Coroll.II). ${ }^{51}$ Nun wird Masse nach Newtons Def.1 der Principia Mathematica als Produkt aus Dichte und Volumen bestimmt. Da jedoch Körper mit demselben Volumen verschieden große Massen enthalten können, ist die spezifische Dichte der Körper bei der Bestimmung der Größe ihrer Masse entscheidend. Wenn die Masse der Körper wiederum der Größe ihrer Trägheitskraft entspricht, kann auch die spezifische Verschiedenheit der Dichte der Körper ohne eine spezifische Verschiedenheit der Trägheit ihrer Elemente nicht erklärt werden (Prop.XII). ${ }^{52}$ Im Neuen Lehrbegriff der Bewegung und Ruhe (1758) zieht Kant die Legitimität des Begriffs der Trägheitskraft erstmals in Zweifel. Kant räumt der Kraft nicht mehr den Status einer "inneren Naturkraft" ein. Gleichwohl erfülle diese nurmehr 'angenommene Kraft' insofern eine heuristische Funktion, als sie "ungemein geschickt" dazu diene, "alle Bewegungsgesetze sehr richtig und leicht daraus herzuleiten." ${ }^{53}$ Bei seiner Kritik der Trägheitskraft geht Kant von dem 'neuen Lehrbegriff' aus, wonach die Begriffe der Bewegung und Ruhe "niemals in absolutem Verstande", sondern "immer respective" zu gebrauchen seien. ${ }^{54}$ An einem Gedankenexperiment versucht er dann zu erläutern, daß ein Körper B, der im Hinblick auf einen sich nähernden Körper A (bis zum Stoß) von außen als ruhig betrachtet wird, doch als bewegt gelten muß, wenn man die Körper in der Binnenperspektive betrachtet. "Denn ihre Beziehung ist gegenseitig, die Veränderung derselben also auch." ${ }^{15}$ Geht man nun

48 Vgl. die "Erläuterungen" zum Specimen Dynamicum in Leibniz (1982) 94f.

49 Jammer (1981) 89. Zu Kants Übernahme und Kritik der Trägheitskraft in den Publikationen der Jahre 1747 bis 1786 s. Jammer 86-89.

50 AA I $485=$ WW I 552/553-554/555.

51 AA I 485f. = WW I 554/555.

52 AA I $486=$ WW I 556/557.

53 AA II $10=$ WW I 574. Vgl. Jammer (1981) 87.

54 AA II 17 = WW I 571: "Ich soll niemals sagen: Eine Körper ruhet, ohne dazu zu setzen, in Ansehung welcher Dinge er ruhe, und niemals sprechen, er bewege sich, ohne zugleich die Gegenstände zu nennen, in Ansehung deren er seine Beziehung ändert."

55 AA II $17=$ WW I 571. 
davon aus, "daß, was man fälschlich vor eine Ruhe in Ansehung des stoßenden Körpers gehalten hat, in der Tat beziehungsweise auf ihn eine Bewegung sei: so leuchtet von selber ein, daß diese Trägheitskraft ohne Not erdacht sei und bei jedem Stoße eine Bewegung eines Körpers gegen einen andern mit gleichem Grade ihm entgegen bewegten angetroffen werde, welches die Gleichheit der Wirkung und Gegenwirkung, ohne eine besondere Art der Naturkraft erdenken zu dörfen, ganz leicht und begreiflich erkläret." ${ }^{56}$

In den $M A N W$ schließlich verwirft Kant den Begriff einer Trägheitskraft vollends: Sofern eine Kraft immer bewegende Kraft ist, ist die Benennung der Trägheitskraft in sich widersprüchlich und "ein Wort ohne alle Bedeutung" (118). Die Trägheit der Materie, als bloßes Unvermögen, sich von selbst zu bewegen, kann nicht Ursache eines Widerstandes sein. "Einer Bewegung kann nichts widerstehen, als entgegengesetzte Bewegung eines anderen, keineswegs aber dessen Ruhe." (118)

Den Begriff der Masse, ursprünglich noch mit dem der Größe der Trägheitskraft identifiziert, definiert Kant nun als "die Menge des Beweglichen in einem bestimmten Raum." (101) Diese Definition ist für Kant allerdings "ein merkwürdiger und Fundamentalsatz der allgemeinen Mechanik", da die Masse, als Quantität der Substanz im Beweglichen (105) nur in extensiven Größen meßbar ist.

"Denn dadurch wird angezeigt: daß Materie keine andere Größe habe, als die, welche in der Menge des Mannigfaltigen außerhalb einander besteht, folglich auch keinen Grad der bewegenden Kraft mit gegebener Geschwindigkeit, der von dieser Menge unabhängig wäre und bloß als intensive Größe betrachtet werden könnte".(104)

Obgleich Kant den Massenbegriff dynamisch interpretiert, kann

"die Quantität der Substanz an einer Materie nur mechanisch, d.i. durch die Quantität der eigenen Bewegung derselben, und nicht dynamisch, durch die Größe der ursprünglich bewegenden Kräfte, geschätzt werden".(106)

Masse, letztlich auf dem Begriff der Kraft beruhend, ist in quantitativer Hinsicht (durch experimentelle Messungen) nur in extensiven Größen bestimmbar. An dieser Diskrepanz werden die Schwierigkeiten deutlich, die mit der Einführung des metaphysischen Begriffs von Grundkräften zur Interpretation des Massenbegriffs einerseits und der Umsetzung der quantitativen Aspekte dieses Theorems in der Mechanik andererseits verbunden sind. Kants Argument, nach dem diese Diskrepanz gerade in der Konsequenz der Begründungsabsicht der MANW liege, soll in drei Schritten dargestellt werden:

(a) Kant bindet die spezifische Verschiedenheit der Materien an ihre Dichte bzw. spezifische Schwere, zurückführbar auf die Verbindung zweier bewegender Grundkräfte. Gemessen werden die Kräfte an den Wirkungen, also an den durch sie verursachten Widerständen, z.B. am Moment der Schwere oder des Gewichts. Kunde von dem 'eigentlich Empirischen' haben wir in der Empfindung durch die Affektion der äußeren Sinne. Diese Sinne werden durch die 'Mitteilung der Bewegung' mechanisch affiziert, wobei das Affizierte und das Affizierende dadurch als beweglich vorgestellt werden und durch ihre Bewegung aufeinander wirken können, daß ihnen jeweils ursprünglich bewegende Kräfte zugeschrieben werden:

56 AA II $20=$ WW I 574. 
"eine Materie, als bewegt, kann keine bewegende Kraft haben, als nur vermittelst ihrer Zurückstoßung und Anziehung, auf welche und mit welchen sie in ihrer Bewegung unmittelbar wirkt und dadurch ihre eigene Bewegung einer anderen mitteilt."(100)

(b) Newtons Definition der Masse als Produkt aus Dichte und Volumen führt Kant gar nicht an, was zunächst überraschen mag. ${ }^{57}$ Zwar ist für Newton Dichte gegenüber der Masse primär, doch verbindet er Dichte mit der Trägheitskraft, deren Begriff Kant verwirft. Sollen nun die Massen (un)gleichartiger Materien bestimmt werden, so kann dies nur durch Vergleich der Quantität der Bewegung der Materie bei gegebener Geschwindigkeit durchgeführt werden. Die Konstruktion der Quantität der Bewegung ist die Zusammensetzung vieler einander gleichgeltender Bewegungen. (102) Die Differenz der Quantität der Bewegungen läßt sich dann durch das Gewicht, das proportional zur Quantität der Materie ist, bestimmen. ${ }^{58}$

(c) In diesem Zusammenhang macht Kant auf "etwas Befremdliches"(105) aufmerksam: Wenn die Quantität der Materie durch die Quantität der Bewegung mit gegebener Geschwindigkeit und wiederum die Quantität der Bewegung bei derselben Geschwindigkeit durch die Quantität der Materie geschätzt werde, scheine dies die Ableitung zweier identischer Begriffe voneinander $\mathrm{zu}$ fordern. Doch sei das bloß ein vermeintlicher Zirkel, da einerseits die Erklärung eines Begriffs, andererseits die Erklärung der Anwendung des Begriffs auf Erfahrung vorliege.

"Die Quantität des Beweglichen im Raume ist die Quantität der Materie; aber diese Quantität der Materie (die Menge des Beweglichen) beweiset sich in der Erfahrung nur allein durch die Quantität der Bewegung bei gleicher Geschwindigkeit (z.B. durchs Gleichgewicht)."(105)

In dieser Äußerung treten deutlich die Schwierigkeiten hervor, auf die das Projekt der metaphysischen Begründung der empirischen Wissenschaft von der Natur stoßen muß, nämlich - wie Jammer formuliert - die Bedeutungsgehalte des in einer Axiomatik definierten Begriffs und des Begriffs, der die experimentell gewonnenen Daten bezeichnet, in Übereinstimmung zu bringen. ${ }^{59}$ Dem Zitat läßt sich auch eine wichtige Implikation der Kantischen Theorie der Erfahrung entnehmen: Der empirische Gehalt des Begriffs der Materie - Jammer spricht von 'Korrelationen mit der Erfahrung' - läßt sich nicht unabhängig von den quantitativen Aspekten, nach denen er mathematisch beschrieben werden kann, darstellen. Die Art, wie Kant diese Forderung bei der Einführung des Theorems der Grundkräfte einzulösen versucht, hat der Kant-Forschung den Konstruktionsbegriff in den MANW zum Problem werden lassen.

57 Vgl. Mudroch (1987) 111f.

58 Mudroch (1987) 112 macht darauf aufmerksam, das Kant aus heutiger Sicht nicht den Begriff der trägen Masse (Vermögen eines Körpers, der eigenen Beschleunigung zu widerstehen), sondern den Begriff der schweren Masse (Vermögen eines Körpers, Beschleunigung in einem anderen Körper zu bewirken) benutzt. Dies zeigt sich erstens an der Kennzeichnung der ursprünglich bewegenden Kräfte als solcher, "womit die Materie [...] in andere wirkt" (103); zweitens an dem Rekurs auf eine Erläuterung Newtons (1963) $21 \mathrm{zu}$ Def.1 der Principia, in der es zur Quantität der Materie heißt: "Diese Grösse der Materie werde ich im Folgenden unter dem Namen Körper oder Masse verstehen, und sie wird durch das Gewicht des jedesmaligen Körpers bekannt." (21) Vgl. Jammer (1981) 113: Hier werde "ein rein zufälliger Aspekt der klassischen Physik benutzt, nämlich die Proportionalität der schweren und trägen Masse."

59 Jammer (1981) 120. 


\subsection{Das Konfliktmodell zweier ursprünglich bewegender Kräfte}

In 5.4. und 5.5. sollte angedeutet werden, wie das Projekt der metaphysischen Grundlegung der Newtonschen Mechanik bei einer dynamischen Interpretation des Massenbegriffs ausgeführt werden kann. Dabei zeigte sich eine Diskrepanz zwischen dem intensiv gefaßten Massenbegriff und seiner Schätzung in extensiven Größen. Daran anknüpfend soll nun der Einführung des Konfliktmodells zweier Grundkräfte nach der metaphysisch-dynamischen Erklärungsart nachgegangen werden. Insbesondere ist zu klären, wie die Grundkräfte ihrer Funktion nach in das Problem der Konstruierbarkeit eines auf diese Weise metaphysisch fundierten Materiebegriffs eingebunden sind.

Zunächst fällt auf, daß Kant den Grundkräften selbst nur einen hypothetischen Status einräumt. Schon mit dem im zweiten Grundsatzkapitel skizzierten Weg einer metaphysisch-dynamischen Erklärungsart will Kant keineswegs behaupten, "daß dieses wirklich mit der Verschiedenheit der Materien, ihrer spezifischen Schwere nach, so bewandt sei"; ihm gehe es zunächst darum, die Möglichkeit einer solchen Erklärungsart überhaupt auszuweisen. (A174f. B216) Deshalb sei man auch nicht befugt, die Grundkräfte "als wirklich anzunehmen", da ihre Möglichkeit nicht völlig gewiß sei.(84)

Die Grundkräfte seien "von ganz verschiedener Art"(70), weder aufeinander reduzierbar noch von einer höheren Kraft ableitbar(68f.) und eben darum unbegreiflich. Dagegen sei "ihre Vereinigung [!] im Begriffe einer Materie überhaupt" a priori beweisbar(66f.). Die Annahme des leeren Raums kann nicht vermieden werden, wenn überhaupt keine bewegenden Kräfte der Materie vorausgesetzt werden. Denn die Materie erfüllt den Raum nicht durch ihre bloße Existenz ('Solidität'), andernfalls müßte der reale Widerstand der Materie schon aus ihrem Begriffe nach dem Satz des Widerspruchs folgen. (48f., Dyn.Ls.1) Die Annahme des leeren Raumes kann auch nicht umgangen werden unter der Voraussetzung nur einer Kraft, der keine andere Kraft entgegengesetzt gedacht wird. Denn ohne Einschränkung durch Repulsion würde die Materie allein durch Attraktion "in einem mathematischen Punkt zusammenfließen, und der Raum würde leer, mithin ohne alle Materie sein."(66) Umgekehrt würde ohne Einschränkung durch Attraktion die Materie allein durch Repulsion "sich ins Unendliche zerstreuen, und in keinem anzugebenden Raume würde eine anzugebende Quantität Materie anzutreffen sein." (62f., Dyn.Ls.5) Erst auf der Basis eines Konfliktmodells zweier ursprünglich bewegender Kräfte wird der dynamische Begriff der Materie darstellbar (66f.). Aus der Attraktion, "in Verbindung" mit der entgegenwirkenden Repulsion müßte die "Einschränkung" der Repulsion, "mithin die Möglichkeit eines in einem bestimmten Grade erfülleten Raumes, abgeleitet werden können"(74).

Sofern sich ein Gesetz des Verhältnisses, in dem Attraktion und Repulsion "in verschiedenen Entfernungen der Materie und ihrer Teile von einander" stehen, angeben läßt, ist also auch "der dynamische Begriff der Materie, als des Beweglichen, das seinen Raum (in bestimmtem Grade) erfüllt," konstruierbar.(74) Da dabei nur mathematische Größen zu berücksichtigen sind, ist dies "eine rein mathematische Aufgabe"(75), die Kant, 
wie er zugibt, nicht zu lösen vermag. Die $M A N W$ kommen zwar schon dann an ihr Ziel, wenn der Nachweis gelingt, daß die Grundbegriffe der Mechanik dynamisch interpretiert werden können:

"Dies ist nun alles, was Metaphysik zur Konstruktion des Begriffs der Materie, mithin zum Behuf der Anwendung der Mathematik auf Naturwissenschaft, in Ansehung der Eigenschaften, wodurch Materie einen Raum in bestimmtem Maße erfüllet, nur immer leisten kann, nämlich diese Eigenschaften als dynamisch anzusehen und nicht als unbedingte ursprüngliche Positionen, wie sie etwan eine bloß mathematische Behandlung postulieren würde."(99)

Solange aber eine einwandfreie mathematische Darstellung des Verhältnisses, in dem Attraktion und Repulsion stehen, nicht gelingt, ist die Anwendung eines dynamisch fundierten Materiebegriffs auf äußere Erfahrung nicht möglich, auch wenn Kant die Verantwortung "in der Ausführung"(75) der Konstruktion in den Bereich der Mathematik verlegt. Wenn die Erfüllung des Raums als dynamische Eigenschaft der Materie, beschrieben durch das Konfliktmodell zweier Grundkräfte, keine bloße Definition, sondern in der Erfahrung "durch die Quantität der Bewegung bei gleicher Geschwindigkeit (z.B. durchs Gleichgewicht)" (105) nachweisbar sein soll, muß Kant an der Konstruktion gelegen sein. Deshalb kann er nicht umhin, doch noch "eine kleine Vorerinnerung zum Behufe des Versuchs einer solchen vielleicht möglichen Konstruktion" beizufügen. $(76)^{60}$

Bei der Formulierung der dynamischen Interpretation des Massenbegriffs und dem Aufweis seiner Konstruierbarkeit geht Kant von der Kritik an der rationalistischen Ontologie aus. Nach dieser Konzeption sei zur Erklärung der Raumerfüllung "an jedem Dinge, was existiert (Substanz)", die Eigenschaft der "Solidität" anzunehmen.(49) Das Dasein von etwas Realem im Raum als Widerstand gegen anderes folgt dabei als Relation aus den Relaten nach dem Satz des Widerspruchs schon aus dem Begriff der Materie. Mit der mathematisch-mechanischen Erklärungsart werde dann die Solidität "als ein erstes Datum der Konstruktion des Begriffs einer Materie, welches sich selbst nicht weiter konstruieren lasse, angenommen."(49) Vom Standpunkt des Mathematikers ist dieses Datum jedoch beliebig gewählt; man sei deswegen nicht befugt, es "für etwas aller mathematischen Konstruktion ganz Unfähiges zu erklären, um dadurch das Zurückgehen zu den ersten Prinzipien in der Naturwissenschaft zu hemmen."(49) In der Aufdeckung der ersten Prinzipien bestehe gerade die Aufgabe der $M A N W$, um "die Naturphilosophie, so weit als es immer möglich ist, auf die Erforschung der dynamischen Erklärungsgründe zu leiten, weil diese allein bestimmte Gesetze, folglich wahren Vernunftzusammenhang der Erklärungen, hoffen lassen."(98f.) Worin dieser von Kant in Aussicht gestellte 'wahre Vernunftzusammenhang' besteht, ist nun näher auszuführen.

In der metaphysischen Dynamik wird "der Stoff selbst in Grundkräfte verwandelt"(85), die wiederum als 'wesentliche Eigenschaften' gelten sollen.(62f., 66) Die dynamische Erklärung des Begriffs der Materie bestimmt die Grundkraft als eine Eigenschaft, "die sich als Ursache auf eine Wirkung bezieht, nämlich das Vermögen, einer Bewegung innerhalb eines gewissen Raumes zu widerstehen".(47) Attraktion und Repulsion sind solche bewegende Kräfte, wodurch eine Materie Ursache sein kann, der Entfernung einer anderen

60 Zum Versuch, das Verhältnis der Grundkräfte als konstruierbar auszuweisen, vgl. Mudroch (1987) 101105, Brittan (1978) 140-142. 
Materie von ihr bzw. der Annäherung zu ihr zu widerstehen.(49f.) Sofern die bewegende Kraft die Ursache von Bewegung ist, kann die Grundkraft als die Kausalität von etwas beschrieben werden. Denn Kausalität ist der Begriff von derjenigen Eigenschaft von etwas, durch die verstehbar ist, daß etwas eine Ursache ist, d.h. etwas bewirkt.

"Nur alsdann, wenn ich dem, was einen Raum einnimmt, eine Kraft beilege, alles äußere Bewegliche, welches sich annähert, zurück zu treiben, verstehe [!] ich, wie es einen Widerspruch enthalte, daß in den Raum, den ein Ding einnimmt, noch ein anderes von derselben Art eindringe."(49)

Diese hier von Kant ins Spiel gebrachte hermeneutische Aspekt der Einführung seines Kraftbegriffs soll im Ausgang von Cramers Analysen zur Kantischen Theorie der Kausalität erläutert werden. Die Kausalität einer Ursache für Bewegung oder Veränderung ist "diejenige Eigenschaft einer Ursache, aus der erklärt werden kann, daß eine Veränderung geschieht und in der bestimmten Weise geschieht." ${ }^{61}$ Wenn also die Grundkraft die Eigenschaft ist, für eine bestimmte Bewegung oder Veränderung Kausalität $z u$ haben, dann ist die Grundkraft die Eigenschaft, 'die sich als Ursache auf eine Wirkung bezieht', d.h. die Eigenschaft der Ursache für eine Bewegung oder Veränderung.

Nun steht die Grundkraft durch die Eigenschaft, Ursache für etwas zu sein, unter dem Kausalitätsprinzip, das mit unbedingter Allgemeinheit fordert, 'daß alles, was geschieht ("anhebt zu sein"), etwas voraussetzt, worauf es nach einer Regel folgt'.(A189) Das Kausalitätsprinzip legt zwar fest, $d a \beta$ für jede Veränderung etwas vorauszusetzen ist, das für ihren Eintritt Kausalität hat, d.h. daß es eine Ursache gibt. Das Kausalitätsprinzip legt jedoch nicht fest, wie die Kausalität der Ursache für die Veränderung bestimmt ist. ${ }^{62}$

\footnotetext{
"Wie nun überhaupt etwas verändert werden könne; wie es möglich sei, daß auf einen Zustand in einem Zeitpunkte ein entgegengesetzter im anderen folgen könne: davon haben wir a priori nicht den mindesten Begriff. Hierzu wird die Kenntnis wirklicher Kräfte erfordert, welche nur empirisch gegeben werden kann, z.B. der bewegenden Kräfte, oder, welches einerlei ist, gewisser sukzessiver Erscheinungen, (als Bewegungen), welche solche Kräfte anzeigen." (A206f. B252)
}

Das Kausalitätsprinzip formuliert nur eine Metaregel, nach der "Regeln der Verknüpfung des Vor und Nach von Zuständen an Dingen" aufgefunden werden. ${ }^{63}$ Diese Regeln sind als besondere Kausalgesetze empirisch aufzufinden.

Nun ist klar, daß uns die ursprünglich bewegenden Kräfte nicht für sich (einzeln), sondern nur in Verbindung miteinander - im "Widerspiel"(69) als "Einschränkung" der Repulsion durch Attraktion(74) - gegeben sind. Aus dieser Einschränkung folgt "die Möglichkeit eines in einem bestimmten Grade erfülleten Raumes"(74). Daß Raumerfüllung in einem bestimmten Grade vorliegt, kann der Mathematiker dann "als ein erstes Datum der Konstruktion des Begriffs einer Materie" annehmen (49), und zwar unabhängig von den metaphysischen Implikationen, die das beschriebene Phänomen erst verstehbar machen. Daran gemessen, beschreibt Kant die Kompetenz und Grenzen der Metaphysik so: "sie [die Metaphysik] verantwortet bloß die Richtigkeit der unserer Vernunfterkenntnis vergönneten Elemente der Konstruktion, die Unzulänglichkeit und die Schranken unserer Vernunft in der Ausführung verantwortet sie nicht." (75) Es ist die Aufgabe der MANW, die

\footnotetext{
61 Cramer (1985) 246.

62 Ebd.

63 Ebd.
} 
Möglichkeit der Konstruktion von Dichtigkeit überhaupt aufzuweisen mit Rekurs auf die in der $K r V$ entwickelten Kriterien und Prinzipien als einer "hier methodisch gebrauchten Metaphysik"(84). Dagegen können die $M A N W$ a priori keine Aussage über die Bestimmtheit der Dichte zu einem bestimmten Zeitpunkt machen. Dies aufzufinden, ist Aufgabe der empirischen Wissenschaft von der Natur.

Die Form jeder Veränderung und die Bedingungen, unter denen die Veränderung eintritt, können - unabhängig vom Inhalt derselben - a priori erwogen und antizipiert werden. Was jedoch nicht a priori antizipierbar ist, sind erstens die spezifische Bestimmtheit von etwas bzw. die Bestimmtheit der Kausalität von etwas und zweitens die Tatsache, daß ein Wechsel von Zuständen an etwas überhaupt stattfindet.

\footnotetext{
"Wenn nun alle Erscheinungen, sowohl extensiv, als intensiv betrachtet, kontinuierliche Größen sind, so würde der Satz: daß auch alle Veränderung (Übergang eines Dinges aus einem Zustande in den anderen) kontinuierlich sein, leicht und mit mathematischer Evidenz hier bewiesen werden können, wenn nicht die Kausalität einer Veränderung überhaupt ganz außerhalb den Grenzen einer Transzendental-Philosophie läge, und empirische Prinzipien voraussetzte. Denn $d a \beta$ eine Ursache möglich sei, welche den Zustand der Dinge verändere, d.i. sie zum Gegenteil eines gewissen gegebenen Zustandes bestimme, davon gibt uns der Verstand a priori gar keine Eröffnung nicht bloß deswegen, weil er die Möglichkeit davon gar nicht einsieht [...] sondern weil die Veränderlichkeit nur gewisse Bestimmungen der Erscheinungen trifft, welche die Erfahrung allein lehren kann, indessen $\mathrm{da} ß$ ihre Ursache in dem Unveränderlichen anzutreffen ist."(A171 B212f.)
}

Das Kausalitätsprinzip besagt nur, daß ein Wechsel an Zuständen von Dingen abläuft und daß dieser Wechsel eine Ursache hat. Der Verstand "sieht aber die Möglichkeit einer Ursache hierfür nicht a priori ein, weil er die Möglichkeit des Wechsels der Erscheinungen selber nicht einsieht."64 An der materiellen Differenz in der Sinnlichkeit sind die Bedingungen, unter denen die Differenz auftritt, a priori herleitbar und antizipierbar. Diese Differenz selbst kann nur durch Rekurs auf ein empirisches Datum gegeben werden. ${ }^{65}$ Daraus wird einsichtig, daß sich der logische Inhalt von etwas, das unter dem Kausalitätsprinzip steht, auch nur im Rekurs auf ein empirisches Datum ausreichend angeben läßt. Dies gilt auch für den Begriff der Kraft. Sätze a priori, in denen dieser Begriff als Subjekt oder Prädikat auftritt, sprechen von etwas, das sich nur mit Bezug auf etwas exponieren läßt, was in der empirischen Anschauung als ein Datum, dem Empfindung korrespondiert, gegeben ist. Diese Sätze antizipieren nicht das Datum selbst, sondern formulieren die Bedingung, unter der das Datum auftritt und auf einen Gegenstand der Erfahrung bezogen werden kann.

Sofern mit dem Begriff der ursprünglich bewegenden Kraft die Eigenschaft von etwas bezeichnet ist, Ursache für eine Veränderung zu sein, hat dieses Etwas Kausalität für etwas anderes, d.h. hier "das Vermögen, einer Bewegung innerhalb eines gewissen Raumes zu widerstehen"(47). Die Erfüllung des Raumes entspricht der Einschränkung der Repulsion durch Attraktion, wodurch "die Möglichkeit eines in einem bestimmten Grade erfülleten Raumes"(74), der prinzipiell konstruierbar sein soll, angezeigt wird. Mithin sind die Grundkräfte die Bedingung der Möglichkeit der Raumerfüllung einer Materie.

64 Ebd. 248; vgl. 244f.

65 Ebd. 248 
Da Raumerfüllung nicht unbezogen auf empirische Anschauung gegeben werden kann, sagt der Begriff der Grundkraft bloß die Synthesis der empirischen Anschauung aus, also von etwas, das nur a posteriori gegeben werden kann. Diese Synthesis ist keine Synthesis a posteriori, da das, was durch sie verbunden wird, so notwendig verbunden wird. Da jedoch das Verbundene nur im Rekurs auf empirische Daten als Mannigfaltigkeit von Empfindungen gebbar ist, ist die Synthesis in diesem Sinn auch keine reine Synthesis a priori. Eine solche Synthesis wäre rein, "wenn das Mannigfaltige nicht empirisch, sondern a priori gegeben ist (wie das im Raum und der Zeit)."(A77 B103)

Eine solche Bedingung erfüllt die mathematische Synthesis, nach der ein Zahlbegriff oder geometrischer Begriff in der reinen Anschauung a priori dargestellt, d.h. konstruiert werden kann. Gerade darin aber unterscheidet sich ein mathematischer von einem transzendentalen Satz:

"Also ist ein transzendentaler Satz ein synthetisches Vernunfterkenntnis nach bloßen Begriffen, und mithin diskursiv, indem dadurch alle synthetische Einheit der empirischen Erkenntnis allererst möglich, keine Anschauung aber dadurch a priori gegeben wird."(A722 B750)

Ein transzendentaler Satz zeichnet sich dadurch aus, daß durch ihn eine Synthesis a priori der empirischen Anschauung ausgedrückt ist. Da die empirische Anschauung nur als Mannigfaltiges gegeben wird, das nicht rein, sondern empirisch ist, ist diese Synthesis eine nicht-reine Synthesis a priori. ${ }^{66}$ Die Begriffe von Grundkräften sind als Bedingung der Möglichkeit von Raumerfüllung selber transzendentale Begriffe, die den Status von Begriffen einer nicht-reinen Synthesis a priori haben. Durch die Grundkräfte wird die Qualität von etwas (spezifische Dichte) überhaupt denkbar als das Reale, das in der Verbindung der Kräfte graduell bestimmbar ist. Doch gilt hierbei, daß die Qualität der Empfindung "jederzeit bloß empirisch" ist und "a priori gar nicht vorgestellt werden" kann.

"Aber das Reale, was den Empfindungen überhaupt korrespondiert, im Gegensatz mit der Negation = 0 , stellt nur etwas vor, dessen Begriff an sich ein Sein enthält, und bedeutet nichts als die Synthesis [!] in einem empirischen Bewußtsein überhaupt."(A175f. B217)

Daß die Begriffe von Grundkräften transzendentale Begriffe sind, ist der Grund dafür, daß sie nicht konstruierbar sind. ${ }^{67}$ Als Grundkräfte treten sie im Kontext von "Prinzipien der Notwendigkeit dessen, was zum Dasein eines Dinges gehört"(13), auf. Als solche sind die Grundkräfte weder in der reinen Anschauung a priori noch in der empirischen Anschauung gebbar.

"Den mathematischen Begriff eines Triangels würde ich konstruieren, d.i. a priori in der Anschauung
geben, und auf diesem Wege eine synthetische, aber rationale Erkenntnis bekommen. Aber, wenn mir
der transzendentale Begriff einer Realität, Substanz, Kraft usw. gegeben ist, so bezeichnet er weder
eine empirische, noch reine Anschauung, sondern lediglich die Synthesis der empirischen
Anschauungen (die also a priori nicht gegeben werden können), und es kann also aus ihm, weil die

${ }^{66}$ Der Status, Synthesis a priori zu sein, ist nicht abhängig davon, daß das Mannigfaltige als das reine Mannigfaltige der Anschauungsformen von Raum und Zeit gegeben wird. Diese These ist erstmals von Cramer (1985) 214f. vertreten worden.

67 "[...] wenn der Stoff selbst in Grundkräfte verwandelt wird (deren Gesetze a priori zu bestimmen, noch weniger aber eine Mannigfaltigkeit derselben, welche zu Erklärung der spezifischen Verschiedenheit der Materie zureichte, zuverlässig anzugeben, wir nicht im Stande sind), uns alle Mittel abgehen, diesen Begriff der Materie zu konstruieren, und, was wir allgemein dachten, in der Anschauung als möglich darzustellen."(85) 
Synthesis nicht a priori zu der Anschauung, die ihm korrespondiert, hinausgehen kann, auch kein bestimmender synthetischer Satz, sondern nur ein Grundsatz der Synthesis möglicher empirischer Anschauungen entspringen."(A722 B750.)

Mit dem Begriff von Grundkräften ist zweifellos die Synthesis empirischer Anschauungen bezeichnet. Ein Satz, in dem ein solcher Begriff auftritt, ist der Grundsatz der Synthesis möglicher empirischer Anschauungen. Einen Grundsatz dieser Art formuliert Kant tatsächlich in den MADyn:

"Das allgemeine Prinzip der Dynamik der materiellen Natur ist: daß alles Reale der Gegenstände äußerer Sinne, die das, was nicht bloß Bestimmung des Raums (Ort, Ausdehnung und Figur) ist, als bewegende Kraft angesehen werden müsse".(82f.)

Dieses Prinzip ist kein bestimmender synthetischer Satz, aber ein synthetisches Urteil a priori. Der in ihm auftretende Begriff der bewegenden Kraft bezeichnet eine Synthesis empirischer Anschauungen, das Urteil selbst ist also nicht unabhängig von empirischen Daten der Sinnlichkeit explizierbar, mithin ein nicht-reines synthetisches Urteil a priori. ${ }^{68}$ Deshalb gelingt es nicht, die Gesetze der Grundkräfte in Verbindung miteinander "a priori zu bestimmen, noch weniger aber eine Mannigfaltigkeit derselben, welche zu Erklärung der spezifischen Verschiedenheit der Materie zureichte, zuverlässig anzugeben". Und es gelingt ferner nicht, "diesen [dynamischen] Begriff der Materie zu konstruieren, und, was wir allgemein dachten, in der Anschauung als möglich darzustellen."(85) Daß die Konstruktion des dynamischen Begriffs der Materie nicht gelingt, ist freilich kein Mangel, sondern liegt, wie zu zeigen war, gerade in der Konsequenz der metaphysischen Erklärungsart.

\subsection{Bewegung als "Beschreibung eines Raumes"}

$\mathrm{Daß}$ das sog. Solide der Materie als dynamische Eigenschaft zu interpretieren ist und die Erfüllung des Raums in bestimmtem Grad aus der Verbindung von Attraktion und Repulsion resultiert, ist das Ergebnis der "Nachforschung der Metaphysik"(98f.). Da der Begriff der Grundkräfte bloß eine transzendentale Synthesis empirischer Anschauungen bezeichnet, sind diese Kräfte selbst nicht in der reinen Anschauung a priori darstellbar. Dagegen läßt sich aus der Verbindung der Kräfte, nämlich der Einschränkung der Repulsion durch die Attraktion, die Undurchdringlichkeit der Materie als diejenige Eigenschaft rekonstruieren, "wodurch Materie einen Raum in bestimmtem Maße erfüllet".(99) In dieser Bestimmung erschöpft sich allerdings schon, "was Metaphysik zur Konstruktion des Begriffs der Materie, mithin zum Behuf der Anwendung der Mathematik auf Naturwissenschaft [...] nur immer leisten kann".(99) Wenn die Raumerfüllung auf die Verbindung zweier Kräfte zurückgeführt wird, muß jedoch über deren graduelle Bestimmbarkeit hinaus die Mathematisierbarkeit gerade der Dualität von Kräften herleitbar sein. Eine Begründung dafür läßt sich offensichtlich nicht aus der metaphysischen Dynamik selbst gewinnen.

68 Zur Einführung dieses Urteilstyps durch Cramer (1985) vgl. 5.1. 
In der Anmerkung zu Dyn.Erkl.1 verweist Kant darauf, daß die dynamische Erklärung des Begriffs der Materie die phoronomische Erklärung voraussetzt.(47) Demgemäß wird der Beweis zu Dyn.Ls.1, daß die Materie den Raum durch eine besondere bewegende Kraft erfüllt, mit Bezug auf Phor.Ls.1 geführt. Sofern das Eindringen einer Materie in einen Raum, den eine andere Materie erfüllt, Bewegung ist, muß der Widerstand dagegen wiederum als Bewegung gedacht werden, da mit Bewegung nur "eine andere Bewegung eben desselben Beweglichen in entgegengesetzter Richtung (Phoron. Lehrs.)" (48) verbunden werden kann. ${ }^{6}$ Hier ist die Zusammensetzung zweier Bewegungen an demselben Beweglichen beschrieben. Die Dualität der Bewegungen wird durch ihre Orientierung im Raum nach entgegengesetzten Richtungen ermöglicht. Da Bewegung in Geschwindigkeit meßbar und Geschwindigkeit in Vektorenlinien darstellbar ist, erstreckt sich die geometrische Darstellbarkeit auf die Orientierung der Linien nach nur zwei entgegengesetzten Richtungen.

Einerseits formulieren die MAPhor die Bedingungen einer 'rein mathematischen Grundlegung', nach der die Zusammensetzung der Bewegungen "durch physische Ursachen, d.i. Kräfte", beschrieben werden kann.(34) In dieser Hinsicht versteht Kant die Phoronomie als "die reine Größenlehre (mathesis) der Bewegungen"(37, 45). Bewegung wird als ein reines Quantum(22), die Materie unabhängig vom Begriff der Ausdehnung als ein Punkt betrachtet.(27, 37) Die beiden Momente der Beschreibung von Bewegung sind Richtung und Geschwindigkeit(29), die als Größen(25) ihrer Zusammensetzung nach a priori bestimmt werden(34): "so ist die Phoronomie eine Lehre der Zusammensetzung der Bewegungen eben desselben Punktes nach ihrer Richtung und Geschwindigkeit". Dabei wird vorausgesetzt, daß die Bestimmung einer Größe durch Zusammensetzung von Gleichartigem erfolgt, so daß Bewegung nur mit Bewegung zusammengesetzt gedacht werden kann: "Um die Bewegung zu finden, die aus der Zusammensetzung von mehreren [...] entspringt, darf man nur, wie bei aller Größenerzeugung, zuerst diejenige suchen, die unter gegebenen Bedingungen aus zweien zusammengesetzt ist".(37)

Andererseits ist die hier skizzierte 'rein mathematische Grundlegung' eingebettet in MAPhor, deren Aufgabe es ist, die 'erste Anwendung' der Mathematik auf Gegenstände äußerer Sinne zu begründen, und die selbst nicht identisch ist mit der Phoronomie genannten mathematisch-physikalischen Disziplin. Der Begriff der Materie muß so operabel gemacht werden, daß Aussagen von mathematischer Exaktheit über materielle Entitäten, die nicht identisch sind mit geometrischen Entitäten, getroffen werden können. Demnach macht das Beweisprogramm der MAPhor zwei Voraussetzungen:

(a) Geometrische Entitäten als Gegenstände der euklidischen Geometrie unterscheiden sich von materiellen Dingen durch ihre Unbeweglichkeit und sind deshalb keine Gegenstände möglicher Erfahrung. Dies geht aus B155* hervor: "Bewegung eines Objekts im Raume gehört nicht in eine reine Wissenschaft, folglich auch nicht in die Geometrie; weil, daß etwas beweglich sei, nicht a priori, sondern nur durch Erfahrung erkannt werden kann." Kants Argument für den Ausschluß des Begriffs der Bewegung aus der Geometrie beruht

69 "Also ist der Widerstand, den eine Materie in dem Raum, den sie erfüllt, allem Eindringen anderer leistet, eine Ursache der Bewegung der letzteren in entgegengesetzter Richtung."(48) 
darauf, daß Bewegung an etwas Beweglichem wahrgenommen wird, eine Wahrnehmung aber ein empirisches Bewußtsein ist, 'in welchem zugleich Empfindung ist'(B207f.).

(b) Der experimentelle Nachweis der Bewegung bestimmter Naturdinge erfordert deren Mathematisierbarkeit nach raum-zeitlichen Koordinaten (Größe, Dauer, Gestalt), ohne daß dabei mathematische und materielle Entitäten ununterscheidbar wären. Daß etwas beweglich ist, läßt sich nur im Rekurs auf eine Mannigfaltigkeit aussagen, die jedoch nicht schon mit der reinen Anschauung, sondern als empirische Anschauung unter der Bedingung der reinen Anschauung und nur in der äußeren Anschauung gegeben ist, doch so, daß das Ding und der Ort, an dem es ist, unterschieden werden können.

"Denn damit gewisse Empfindungen auf etwas außer mich bezogen werden, (d.i. auf etwas in einem
anderen Orte des Raumes, als darinnen ich mich befinde), imgleichen damit ich sie als außer- und
nebeneinander, mithin nicht bloß verschieden, sondern als in verschiedenen Orten vorstellen könne,
dazu muß die Vorstellung des Raumes schon zum Grunde liegen."(A23 B38)

Im Anschluß an Cramer ${ }^{70}$ soll nun Kants Argument für die Unbeweglichkeit geometrischer Entitäten entwickelt werden: Daß ich etwas als 'außer mich' beziehen kann, setzt den Unterschied mindestens zweier Örter voraus, deren Lage in Relation zueinander bestimmbar ist. Ein Ort ist nur relativ zu einem anderen Ort identifizierbar, der seiner Lage nach von ihm durch Abstand verschieden ist, d.h. sich auf etwas beziehen läßt, das außerhalb von ihm liegt. Wäre nun ein Ort beweglich, müßten sich die Örter auch so verschieben lassen, daß sie miteinander zur Deckung gebracht werden. Dann wären die Örter von ihrer Lage her ununterscheidbar. Zugleich könnte so nicht mehr sinnvoll von zwei Örtern gesprochen werden. Ein Ort wäre nur dann beweglich, wenn er selbst an einem Ort ist. Da jedoch kein Ort an einem Ort sein kann, ist kein Ort beweglich.

Nun soll die Materie in der Phoronomie bloß als ein Punkt betrachtet werden. Ein Punkt ist dann beweglich, wenn er von dem Ort, an dem er ist, unterschieden werden kann. Dies trifft jedoch für den geometrischen Punkt gerade nicht zu. Der geometrische Ort ist im Raum ein geometrischer Punkt, der daher auch nicht beweglich sein kann. Aus diesem Grunde schränkt Kant die 'gemeine Erklärung' der Bewegung als Veränderung des Orts folgendermaßen ein: "Nur von einem beweglichen, d.i. physischen Punkte kann man sagen: Bewegung sei jederzeit Veränderung des Orts."(28)

Da der phoronomische Begriff der Materie aber allgemeingeltend, "mithin auch auf bewegte Körper passend" sein soll, und der Ort eines jeden Körpers ein Punkt ist(28), führt Kant in Phor.Erk1.2 eine andere Definition der Bewegung ein: "Bewegung eines Dinges ist die Veränderung der äußeren Verhältnisse desselben zu einem gegebenen Raum."(27) Danach gilt etwas, das nicht identisch ist mit der reinen Mannigfaltigkeit im Raum (Punkt, Linie, Fläche, Körper), genau dann als beweglich, wenn es unter Wahrung seiner Identität während der Veränderung seine äußeren Verhältnisse $\mathrm{zu}$ einem gegebenen Raum verändern kann. ${ }^{71}$ Ausdruck dafür, daß Bewegung nicht unabhängig von raum-zeitlichen Koordinaten erfaßt werden kann, ist die physikalische Messung der Bewegung durch die Größe der Geschwindigkeit, die als Quotient aus Strecke pro Zeiteinheit definiert ist. Da

71 Ebd. 99. 
Bewegung die Grundbestimmung der Gegenstände äußerer Sinne ist, gebraucht Kant Geschwindigkeit in den MAPhor auch "bloß in räumlicher Bedeutung"(31).

$\mathrm{Daß}$ Mathematik überhaupt auf Erfahrung anwendbar ist, ist nach dem Entwurf der transzendentalen Theorie der Erfahrung insoweit begründet, als die Bedingungen der Möglichkeit der Geometrie solche der Erfahrung der Gegenstände äußerer Sinne sind. Diese Überlegung liegt jedenfalls auch der Konzeption der MAPhor zugrunde:

"In der Phoronomie [...] kann die Bewegung nur als Beschreibung eines Raumes betrachtet werden, doch so, daß ich nicht bloß, wie in der Geometrie, auf den Raum, der beschrieben wird, sondern auch auf die Zeit darin, mithin auf die Geschwindigkeit, womit ein Punkt den Raum beschreibt, Acht habe."(37)

Mit der Bestimmung der phoronomischen Betrachtungsweise von Bewegung als 'Beschreibung eines Raumes' greift Kant auf einen Fachausdruck der Geometrie zurück. Nach dem Verfahren der Tafelgeometrie wird (mit dem Lineal) eine gerade Linie 'gezogen' im Ausgang von einem Punkt A zu einem festgelegten Punkt B; oder es wird ein Zirkel 'beschrieben', indem (mit dem Zirkel) ein Kreis 'geschlagen' wird. Dieses Verfahren darf jedoch nur als eine empirische Darstellung eines im Begriff der Linie oder des Kreises angezeigten geometrischen Sachverhalts gedeutet werden. Denn Kant zufolge ist im Raum der euklidischen Geometrie eben nichts Bewegliches, wie auch die Elemente des Euklid keine genetische Definition von Linie und Kreis vorsehen: Nach Def.2 des ersten Buches ist eine Linie nicht ein bewegter Punkt, sondern "eine Länge ohne Breite". ${ }^{72}$

Die phoronomische Betrachtung von Bewegung als "Beschreibung eines Raumes" ist daher nicht identisch mit der entsprechenden Konstruktion geometrischer Räume auf der Grundlage genetischer Definitionen. Damit ist vielmehr auf eine Handlung des Subjekts verwiesen, "dadurch wir den inneren Sinn seiner Form gemäß [sukzessiv] bestimmen" (B154f.): "Bewegung, als Beschreibung eines Raumes, ist ein reiner Aktus der sukzessiven Synthesis des Mannigfaltigen in der äußeren Anschauung überhaupt durch produktive Einbildungskraft, und gehört nicht allein zur Geometrie, sondern sogar zur Transzendentalphilosophie."(B155*) Mit dem Rekurs auf die transzendentale Handlung der Einbildungskraft gelingt es Kant, für die Phoronomie im Sinne einer Kinematik eine innere Verbindung von Geometrie und Zeitbegriff ${ }^{3}$ herzustellen. So stellt das 'Ziehen einer geraden Linie die äußerliche figürliche Vorstellung der Zeit' dar.(B154, vgl.B291f.) Dies erlaubt eine temporale Interpretation der in räumlicher Darstellung erscheinenden Größen der Geschwindigkeit: "Da alle diese Bewegungen als zugleich geschehend betrachtet werden, so ergibt sich aus dem Verhältnis der Linien, d.i. der beschriebenen Räume der Bewegung, in gleicher Zeit, so fort auch das Verhältnis der Geschwindigkeit." (38)

Der innere Sinn hat in der Kantischen Theorie der Erfahrung eine basale Funktion, in der er gleichermaßen für die mathematische Erkenntnis $^{74}$ wie für die Erfahrbarkeit der

Vgl. Cramer (1985) 360-363.

73 Vgl. Jammer (1981) 95.

74 "Geometrie legt die reine Anschauung des Raumes zum Grunde. Arithmetik bringt selbst ihre Zahlbegriffe durch sukzessive Hinzusetzung der Einheiten in der Zeit zustande, vornehmlich aber reine Mechanik kann ihre Begriffe von Bewegung nur vermittelst der Vorstellung der Zeit zustande bringen." $(P \S 10:$ AA IV $283=$ WW III 145) 
Gegenstände äußerer Sinne ${ }^{75}$ konstitutiv ist und damit die Anwendung der Mathematik auf Empirie prinzipiell legitimieren soll. Nun gehen die MAPhor zwar von Betrachtung der Materie als Punkt aus, doch sollen ihre Bestimmungen auf Körper übertragbar sein, deren Existenz damit schon vorausgesetzt wird: "Wenn gleichwohl der Ausdruck eines Körpers hier bisweilen gebraucht werden sollte, so geschieht dies nur, um die Anwendung der Prinzipien der Phoronomie auf die noch folgende bestimmtere Begriffe der Materie gewissermaßen zu antizipieren". (25)

Die objektive phoronomische Erklärung nimmt insofern auf das Äußere Bezug, als sie Materie als das Bewegliche explizit in den Raum versetzt. Vom transzendentalen Standpunkt ist diese Vorstellung als Gegenstand möglicher Erfahrung denkbar nur unter der Annahme zweier Räume, eines materiellen (relativen) Raumes, der selbst beweglich ist, und eines reinen (absoluten) Raumes, der 'schlechterdings unbeweglich' ist (25), wobei sich der materielle Raum in dem oder durch den reinen Raum bewegen können soll. Die Scheidung zweier Räume sei "um der Möglichkeit der Erfahrung willen" vorauszusetzen.(26) Da sie damit auf die transzendentale Reflexion auf die apriorischen und aposteriorischen Elemente einer Vorstellung zurückgeht, hat sie ihre Grundlage in der "bloß metaphysischen Erklärung" des Begriffs der Materie. 


\section{Schellings Projekt einer transzendentalen Konstruktion}

Schelling macht den Begriff der Konstruktion zum universellen Methodenbegriff in der Philosophie. Er greift damit einen Begriff auf, an dem Kant das Spezifikum mathematischer Methodik in der Differenz zur philosophischen festmacht. Schelling gibt der Methode 1797 bei seiner Einführung den Titel 'transzendentale Konstruktion'. Worin viele einen Widerspruch zur Kantischen Konzeption sahen und was sie geradezu als Provokation empfinden mochten, war als Hommage an Kant gedacht. Es war der Versuch, das Unternehmen der Transzendentalphilosophie da zu retten, wo es in den Augen seiner Kritiker "die größte Schwäche" (Reinhold) zeigte. Schelling war wohl der einzige unter den mehr oder weniger berühmten Nachfolgern Kants, der die Bedeutung des Konstruktionstheorems in der $K r V$ erkannte und zur Lösung der offenen Prämissenfrage des kritischen Systems einsetzte.

Der begriffliche Apparat der kritischen Philosophie Kants reicht nicht aus, die Texte idealistischen Philosophierens $\mathrm{zu}$ erschließen. 6.2. soll nach Skizzierung der Forschungslage einige Schwierigkeiten im Umgang mit diesen Texten benennen und die heuristische Perspektive meiner Schelling-Interpretation vorstellen. In 6.1. geht es darum, Schellings Deutung der transzendentalen Fragestellung und, damit einhergehend, die Etablierung des Begriffs der transzendentalen Konstruktion als universellen Methodenbegriff der Philosophie dem Anspruch nach plausibel zu machen.

\subsection{Die Frage nach den 'Bedingungen der Möglichkeit'}

Die transzendentale Nachfrage, wie das Faktum physikalisch zu beschreibender Erfahrung möglich sei, hatte Kant eine Perspektive eröffnet, in der die so erfolgreiche mathematische Naturwissenschaft seiner Zeit ohne eine Grundlegung nach metaphysischen Prinzipien als unhaltbar gelten mußte. Das war der Versuch, Metaphysik gerade da zu retten, wo sie die Konkurrenz um die prägenden Methodenkonzepte längst verloren zu haben schien. Die Umsetzung dieses Programms verlangte den Metaphysikern weniger ab, als der Aufschrei und die Polemik der Kritiker Kants vermuten lassen. Es ging nicht um die Selbstverleugnung der Zunft. Zwar forderte Kant seine Fachkollegen auf,

"ihre Arbeit vorderhand auszusetzen, alles bisher Geschehene als ungeschehen anzusehen und vor allen Dingen zuerst die Frage aufzuwerfen: 'ob auch so etwas als Metaphysik überall nur möglich sei.' [...] $\mathrm{Zu}$ fragen: ob eine Wissenschaft auch wohl möglich sei, setzt voraus, daß man an der Wirklichkeit derselben zweifle." ${ }^{1}$

Doch stellt diese Frage, bezogen auf Metaphysik, nur wer von der Wirklichkeit eben dieser Wissenschaft überzeugt ist. Kants Zweifel ist kein Selbstzweifel. Hinter dem Zweifel steht 
die bare Anerkennung des Faktums, daß wir mit der Physik über einen Typ von Wissenschaft verfügen, der uns eine passable Orientierung in der Welt ermöglicht. Kant ging es ja nicht um den Beweis der Existenz einer Außenwelt, sondern um die Erklärung des bahnbrechenden Erfolgs einer Wissenschaft, die zutreffende, d.h. für jedermann überprüfbare Aussagen über das, was wir Wirklichkeit nennen, zu machen erlaubt.

Für Kant wie Schelling sollte es darauf ankommen, diesem Phänomen eine philosophische Deutung, die der Effizienz der Naturwissenschaft gerecht wurde, zu geben. Wie sehr sich Schelling dabei Kants Sichtweise zu eigen macht, zeigt sich auch daran, wie er in den Ideen zu einer Philosophie der Natur 1797 Kants Ansatz der transzendentalen Fragestellung $^{2}$ aufnimmt:

"Wer in Erforschung der Natur und im bloßen Genuß ihres Reichthums begriffen ist, der fragt nicht, ob eine Natur und eine Erfahrung möglich sey. Genug, sie ist für ihn da; er hat sie durch die That selbst wirklich gemacht, und die Frage, was möglich ist, macht nur der, der die Wirklichkeit nicht in seiner Hand zu halten glaubt. [...] Wie eine Welt außer uns, wie eine Natur und mit ihr Erfahrung möglich sey, diese Frage verdanken wir der Philosophie, oder vielmehr mit dieser Frage entstand Philosophie." ${ }^{3}$

Schelling nimmt die Frage danach, ob und was möglich sei, als Ausweis des Zweifels an der Wirklichkeit selbst. Die zweite Frage nach dem, wie es möglich sei, gründet zwar in der Verunsicherung über das, was wirklich ist, konstituiert jedoch zugleich Philosophie und die philosophische Nachfrage nach ihrer Möglichkeit. Geht es Kant im Ausgang des Philosophierens um die Kompetenz der Metaphysik, so stellt Schelling an den Anfang eine Analyse der Kompetenz des Fragenden selbst:

"Man muß also jener Frage selbst, mit der alle Philosophie beginnt, fähig seyn, um philosophiren zu können. Diese Frage ist nicht eine solche, die man ohne eigenes Zuthun anderen nachsprechen kann. Sie ist ein freihervorgebrachtes, selbst aufgegebenes Problem. Daß ich diese Frage aufzuwerfen fähig bin, ist Beweis genug, daß ich als dieser von äußeren Dingen unabhängig bin; denn wie hätte ich sonst fragen können, wie diese Dinge selbst für mich, in meiner Vorstellung möglich sind." ${ }^{4}$

Schelling schlägt zwei Wege ein, um diesen Ansatz des Fragens verständlich zu machen. Der eine ist die mythologische Version der Vertreibung aus dem Paradies, der die Genese der existentiellen Situation des Fragenden nacherzählt. Der andere deutet die spekulative, später reflexionslogisch genannte Haltung als Weise ihrer philosophischen Bewältigung.

Vor dem Sündenfall, der mit der transzendentalen Nachfrage bezeichnet ist, war der Mensch "im (philosophischen) Naturstande", in dem er, "glücklich in sich selbst" und "unwissend über sich selbst, die Kindheit seiner Vernunft verlebte." Was ihn "verführte" und aus dem "Gleichgewicht der Kräfte und des Bewußtseyns" brachte, war ein Akt der Selbstbefreiung. ${ }^{5}$ Diese Freiheit wurde mit den Mitteln der Reflexion ${ }^{6}$ erkauft, die zur "Trennung", "Entzweiung", "Entfremdung" von Mensch und Natur führte. ${ }^{7}$ "Mit jener Trennung zuerst beginnt die Reflexion; von nun an trennt er was die Natur auf immer

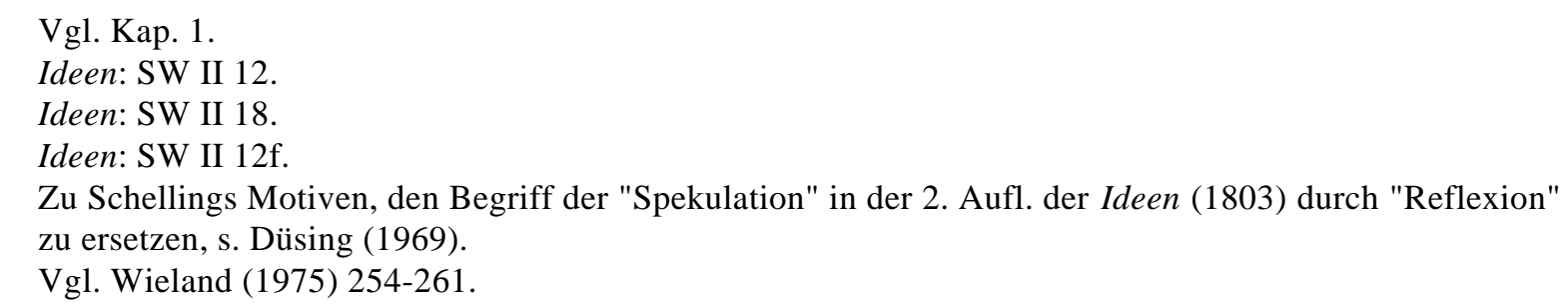


vereinigt hatte, trennt den Gegenstand von der Anschauung, den Begriff vom Bilde, endlich (indem er sein eigenes Objekt wird) sich selbst von sich selbst."8

Wo der Mensch in der bloßen Reflexion verharrt und "jene Trennung zwischen dem Menschen und der Welt permanent [macht]"9, schneidet er sich die Rückkehr in den ursprünglichen Zustand $\mathrm{ab}$, die Emanzipation wird zur Pathogenese, zu einer "Geisteskrankheit des Menschen". ${ }^{10}$ Schelling stellt eine fatale Beziehung zwischen Tätigkeit und Reflexion des Menschen her: "Je weniger er aber über sich selbst reflektirt, desto thätiger ist er. Seine edelste Thätigkeit ist die, die sich selbst nicht kennt. Sobald er sich selbst zum Objekt macht, handelt nicht mehr der ganze Mensch, er hat einen Theil seiner Thätigkeit aufgehoben, um über den anderen reflektiren zu können."11

Was sich hier als der "Irrationalismus Schellings" (Lukács) ankündigt, hat man wohlwollender auch als eine Form von "Esoterik" ausgelegt, die Philosophie als Aktualität einer Tätigkeit, nicht als Resultat im Sinne etwa eines Systems theoretischer Sätze begreift. ${ }^{12}$ Doch hat der durch die transzendentale Fragestellung eröffnete Blick auf die reflexive Struktur des Denkens in den Augen Schellings etwas Zwiespältiges: Reflexion offenbart sich nicht nur als ein Moment der Abspaltung von Tätigem und Denkendem, es dient auch als ein Mittel der Selbstaufklärung. Im Aufwerfen der Frage manifestiert sich die Freiheit des Fragenden: "ich werde durch diese Frage selbst zu einem Wesen, das in Ansehung alles Vorstellens sich ursprünglich frei fühlt [...]. Durch diese Frage selbst werde ich ein Wesen, das, unabhängig von äußeren Dingen, ein Seyn in sich selbst hat." ${ }^{13}$

Das Fragen ist theoretisches Mittel für einen Standpunktwechsel von der gemeinen zur philosophischen Weltansicht, mit dem sich der Fragende von den natürlichen Vorurteilen seiner Orientierung in der Welt befreit. "Wer zuerst darauf achtete, daß er sich selbst von äußeren Dingen, daß er somit seine Vorstellungen von den Gegenständen [...] unterscheiden konnte, war der erste Philosoph." Dagegen gründe "für den gemeinen Verstand die Ueberzeugung von der Realität äußerer Dinge" in der "Unfähigkeit", bei der Vorstellung eines Gegenstandes Akt und Inhalt des Vorstellens zu unterscheiden. Gegenstand und Vorstellung sind für ihn "eins und dasselbe". ${ }^{14}$

"Diese Identität des Gegenstandes und der Vorstellung hebt nun der Philosoph auf, indem er fragt: Wie entstehen Vorstellungen äußerer Dinge in uns? Durch diese Frage versetzen wir die Dinge außer uns, setzen sie voraus als unabhängig von unsern Vorstellungen. Gleichwohl soll zwischen ihnen und unsern Vorstellungen Zusammenhang seyn. Nun kennen wir aber keinen realen Zusammenhang verschiedener Dinge, als den von Ursache und Wirkung. Also ist auch der erste Versuch der Philosophie der: Gegenstand und Vorstellung ins Verhältnis von Ursache und Wirkung zu setzen." ${ }^{15}$

8 Ideen: SW II 13.

9 Ideen: SW II 14.

10 Ideen: SW II 13.

11 Ideen: SW II 13.

12 Wieland (1975) 257: "Dies ist keine Esoterik, die sich auf die Kenntnis bestimmter für wahr gehaltener Aussagen gründet, Aussagen, die nur einem bestimmten Kreis mitgeteilt werden. [...] Schellings Esoterik orientiert sich denn auch an der Fähigkeit, einen Akt der Freiheit auszuführen, und gerade nicht an Sätzen, auch nicht an solchen, die sich ihrerseits auf diese Ausführung beziehen."

13 Ideen: SW II 16.

14 Ideen: SW II 15.

15 Ideen: SW II 15. 
Philosophie bricht die "Grundvorurtheile"16 der gemeinen Weltansicht auf. Was da als Identisches erscheint, wird durch philosophische Analytik als Kausalprinzipiiertes interpretiert. Diesem "ersten Versuch der Philosophie" müsse ein zweiter der "gesunden" Philosophie folgen, das Entzweite wieder auf eine ursprüngliche Identität zurückzuführen. Die Aporien der Kantischen Philosophie, in die die Fragehaltung der Vernunft führt, dienen dann eigentlich dazu, wie Schelling wohl ganz im Sinne Kants bemerkt, "uns über uns selbst aufzuklären." 17

Schelling hätte eine verblüffende Bestätigung seiner Thesen zum transzendentalen Ansatz bei Kant selbst finden können, wäre ihm Kants Brief an Markus Herz vom 21.2.1772 zugänglich gewesen. Darin berichtet Kant, daß er bei seinen "langen metaphysischen Untersuchungen" auf ein Defizit gestoßen sei, dessen Behebung "in der That den Schlüßel zu dem gantzen Geheimnisse, der bis dahin sich selbst noch verborgenen Metaphys:, ausmacht. Ich frug mich [!] nemlich selbst: auf welchem Grunde beruhet die Beziehung desienigen, was man in uns Vorstellung nennt, auf den Gegenstand?"18

Kant legt sich die Frage vor, wie es sich eigentlich verstehen lasse, daß die "intellectualen Vorstellungen" und Begriffe von Gegenständen, die doch "auf unsrer innern Thätigkeit beruhen", tatsächlich mit den vorgestellten Gegenständen übereinstimmten. Diese Korrespondenz - Kant spricht von "Übereinstimmung", "Einstimmung" oder "Konformität" - von Vorstellung und Gegenstand versucht sich Kant als Hypothese durch das Kausalprinzip begreiflich zu machen; danach sei eben die Beziehung von Gegenstand und "Vorstellung in uns" als der von Ursache und Wirkung "gemäß" zu deuten. ${ }^{19}$

Kant wendet ein, daß diese Hypothese allenfalls zu einer Beschreibung, nicht zur Erklärung des Sachverhaltes tauge; denn "unser Verstand" sei "durch seine Vorstellungen weder die Ursache des Gegenstandes, [...] noch der Gegenstand die Ursache der Verstandesvorstellungen (in sensu reali)." ${ }^{20}$ Anhand der Analyse seiner Ausgangsfrage nach der "begreiflichen Beziehung" von Vorstellung und Gegenstand gelingt es Kant dann, die Prämisse des Unternehmens zu präzisieren, zu dessen Lösung einen Vorschlag vorzulegen er rund ein Jahrzehnt später in der Lage war: "Die reinen Verstandesbegriffe müssen [...] in der Natur der Seele zwar ihre Quellen haben, aber doch weder in so ferne sie vom Object gewirkt werden, noch das object selbst hervorbringen." 21

Bemerkenswert ist nun, daß Kant im Brief an Herz das für den Philosophen noch ganz ungeklärte Korrespondenzproblem aus der Sicht des Mathematikers ohne weiteres als gelöst betrachtet. Diese Übereinstimmung von Gegenstand und Vorstellung als real zu behaupten, gehe in der Mathematik an, "weil die objecte vor uns nur dadurch Größen sind und als Größen können vorgestellet werden, daß wir ihre Vorstellung erzeugen können

16 Vgl. System 1800: SW III 343ff. Schelling kann auf eine lange Tradition der Vorurteilslehren der Aufklärung zurückgreifen. Zu G.F. Meiers Theorie der Grundvorurteile vgl. Schneiders (1983) 208-223.

Ideen: SW II 22.

18 AA X 124.

19 AA X 124.

20 AA X 125.

21 AA X 125. - Im Ausgang von Kants "Fragestellung von 1772" untersucht Carl (1989) die Entwicklungsgeschichte der Überlegungen Kants zu einer 'Deduktion der Kategorien' vor 1781. 
[...]. Daher die Begriffe der Größen selbstthätig seyn und ihre Grundsätze a priori können ausgemacht werden." 22 Kant spricht im Brief noch nicht, wie in der $K r V$, von der Konstruktion der Begriffe als Größen; doch wird hier wie dort die Differenz beider Wissenschaften in epistemologischer Hinsicht formuliert.

Was Kant im Brief noch als problematische Differenz beschreibt und erst später im Kontext der transzendentalen Theorie der Erfahrung auch erklären kann, wird für Schelling im Rückblick zum bestimmenden Motiv der Einführung des Konstruktionsbegriffs als Methodenbegriff der Philosophie:

"Durch die Revolution also, welche die Philosophie in unsern Tagen durch Einführung transscendentaler Prinzipien erfahren hat, ist sie zuerst der Mathematik näher gebracht worden; die Methode, welche in der Mathematik längst mit so großem Glück befolgt worden ist, nämlich sich bloß mit ursprünglichen Constructionen zu beschäftigen". ${ }^{23}$

Es ist überraschend, daß Schelling gerade den Konstruktionsbegriff als Ausweis der transzendentalen Methode interpretiert. Und so prominent die Konstruktion als Methodenbegriff der Philosophie Schellings geworden ist, so wenig ist es Schelling doch gelungen, Herkommen und Funktion des Konstruktionsbegriffs in verständlicher Weise darzustellen. Das ehrgeizige Projekt einer philosophischen Konstruktion ist weitgehend Programm geblieben. Schelling hat keine eigenständige Abhandlung vorgelegt, die Terminologie und Methodologie des Verfahrens systematisch und eingängig entfaltet. Erst seine Rezension von Höijers Abhandlung über die philosophische Construction veranlaßt ihn zu einer ausführlicheren Auseinandersetzung mit Kants Konstruktionstheorem in der "Transzendentalen Methodenlehre" der $\mathrm{KrV}$.

So wird Schelling noch 1802 davon sprechen, daß die "Lehre" von der philosophischen Konstruktion "künftig eines der wichtigsten Kapitel in der wissenschaftlichen Philosophie ausmachen" werde. Er räumt ein, daß "die Methode der Construktion" und damit der "Hauptpunkt, auf den die wissenschaftliche Vollendung der Philosophie ankommt", zwar in den Blick genommen, aber noch nicht erreicht sei. ${ }^{24}$ Schon bei der Einführung des Konstruktionsbegriffs in der Übersicht ist Schelling so ungeschickt, als Instanz der Entscheidung über den neuen Methodenbegriff an so etwas wie "das philosophische Organ" ${ }^{25}$ zu appellieren. Jedenfalls behauptet er, "die neue Ansicht der Dinge" sei "evident für jeden, der das Organ dazu hat, (dem das innre ConstructionsVermögen nicht abgeht)" ${ }^{26}$ Schelling muß die in der Folge prompt auch einsetzende Stigmatisierung des Begriffs nicht anders als bei dem nicht minder schillernden Begriff der intellektuellen Anschauung von vornherein in Kauf genommen haben. Doch das, was die Explikation des Konstruktionsbegriffs auch aufgeschlosseneren Interpreten suspekt macht, scheint eher auf eine ganz eigene Begriffsnot bei seiner Vermittlung zu verweisen.

AA X $125 f$.

Übersicht: SW I 447 = AW IV 173.

Konstruktion: SW V 125.

25 Übersicht: SW I 446 = AW IV 172.

26 Übersicht: SW I 447 = AW IV 173. 


\subsection{Die absolute Konstruktion. Forschungsprobleme}

Die Formeln, mit denen Schelling den Begriff der philosophischen Konstruktion zu umschreiben sucht, klingen so verwegen, daß es Kritikern wie Nachahmern nicht schwer gefallen ist, dieses Verfahren als allzu willkürlich und suspekt erscheinen zu lassen. Schelling spricht von "gleichsam einer realen Construction der Seele selbst"27, von der "ursprünglichsten"28 oder "ursprünglichen (transscendentalen) Construction"29, von der "speculativen und historischen Construktion des Christenthums" ${ }^{30}$, von der "religiösen" oder "philosophischen Construktion der Geschichte"31, von der "wissenschaftlichen Construktion des Staats"32, von "empirischer Construktion"33; der Geist "construirt sich selbst" ${ }^{34}$; als "construirende Kraft" sei die Schwerkraft "durch das Licht bestimmt zu reconstruiren" 35 .

Schon für Hegel ist "diese äußerliche und leere Anwendung der Formel" der Konstruktion zu einem bloßen "Formalismus" und zu einer Methode verkommen, "allem Himmlischen und Irdischen, allen natürlichen und geistigen Gestalten die paar Bestimmungen des allgemeinen Schema's aufzukleben, und auf diese Weise Alles einzurangieren". ${ }^{36}$ Hegels herbe Ironie, mit der er "die Einbildung auf die Vortrefflichkeit dieses Universalmittels" karikiert, zeigt, wie weit schon unter den Zeitgenossen die Stigmatisierung des Begriffs reicht, die bis heute fortwirkt.

Im Gegenzug zeichnet sich in der neueren Forschung eine Tendenz ab, Schelling gerade vom Verdacht der Willkür und des Irrationalen $\mathrm{zu}$ befreien. Dabei scheint sich eine Interpretationsrichtung durchzusetzen, die die Logizität des Konstruktionsverfahrens hervorhebt und dies nach Krings unter das Stichwort der "Logogenese (nicht Realgenese)" faßt. ${ }^{37}$ So geht Boenke davon aus, daß Schellings Konzept der spekulativen Physik sich von der Idee der "Möglichkeit einer apriorischen Konstruktion der Natur" leiten lasse, "die - anders als die Kantische - sich nicht damit begnügen muß, die Gesetze der Verbindung von Erscheinungen transzendental zu begründen, sondern imstande ist, die Erscheinungen selber aus ursprünglichen Kräften genetisch im Sinne einer rein logischen Konstruktion zu entwickeln". ${ }^{38}$ Ausführlicher heißt es dazu, Schelling entwerfe

"anhand des Modells der geometrischen Konstruktion [...] eine absolute Konstruktion, in der
natürliche und geistige Erscheinungsformen als reine Funktionen dieser generativen Struktur, als
sichtbare Aspekte also einer und derselben sich zur erscheinenden Darstellung bringenden Struktur
oder Kraft deriviert werden. Die Gestaltungen, welche diese Aspekte im einzelnen annehmen können,
sind darum begreiflich, weil sie nichts anderes als verschiedene Modi derselben absoluten Realität
sind, die sich gemäß einer bestimmten und zwar mathematisch-dialektischen Strukturlogik in den

27 Übersicht: SW I $380=$ AW IV 107.

Übersicht: SW I $448=$ AW IV 174.

29 Übersicht: SW I 451 = AW IV 177.

30 Vorlesungen über die Methode des akademischen Studiums (1802): SW V 304.

31 Ebd.: SW V 307.

Ebd.: SW V 313.

Ebd.: SW V 323.

34 Übersicht: SW I 386 = AW IV 113.

35 System 1801: SW IV 151.

36 Hegel (1807) 37f.

37 Krings (1985) 115; vgl. Krings (1982), Löw (1985), Boenke (1990).

38 Boenke (1990) 318. 
einzelnen Erscheinungsformen darstellt, wobei diese generative Struktur der Erzeugung von Gegenständlichkeiten im erkennenden Denken im Ausgang von dem ungegenständlichen Prinzip der intellektuellen Anschauung rekonstruiert werden kann." ${ }^{39}$

Leider gibt Boenke keine Auskunft darüber, durch welche Funktion sich die geometrische Konstruktion als Modell für eine absolute Konstruktion erweist, noch macht sie nähere Angaben zu der "mathematisch-dialektischen Strukturlogik". Nun geht es der Autorin auch darum, das für uns unklare Methodenkonzept der philosophischen Konstruktion durch alternative Kennzeichnungen faßlicher $\mathrm{zu}$ machen. Dieses Vorgehen ist deswegen irritierend, weil Schelling selbst über eine bunte Palette solcher Kennzeichnungen verfügt. Die im Anschluß an Krings verfochtene Sprachregelung einer "Logogenese (nicht Realgenese)", z.B. im Sinne "einer spekulativen Rekonstruktion der transzendentalen Genesis der Materie"40, vermag so nicht zu überzeugen, gebraucht doch auch Schelling eine ähnliche Begriffsbildung als Interpretament des Konstruktionsbegriffs in den MANW. So wendet Schelling gegen "Kants Dynamik" ein, "daß er [Kant] sich die beiden Kräfte, solange er bloß logisch construirt, ganz rein, sobald es aber zur realen Construktion (zum Treffen selbst) kommt, immer schon mit Materie verbunden denkt". ${ }^{41}$

Boenke erläutert das Verfahren der "rein logischen Konstruktion" als "genetisch" oder "generisch", und sie spricht in diesem Zusammenhang genauer von "deduzieren", "ableiten" oder "derivieren". ${ }^{42}$ Diese Kennzeichnungen erinnern an die von Ende den "ableitenden Konstruktionsbegriff" getaufte "völlige Neuschöpfung des Deutschen Idealismus", die sie Schelling zuschreibt. ${ }^{43}$ Für Ende ist hierbei "ganz deutlich, daß Konstruktion in dem Ableitungsvorgang der Einzelgegebenheiten aus einem Prinzip besteht." ${ }^{44}$ Dabei werde "das Gegebene durch Ableitung aus den Ideen konstruiert". ${ }^{45}$

Es ist jedoch Schelling selbst, der auf das "Mißverständniß" hinweist, "das Construiren in der Philosophie als ein Ableiten [...] anzusehen". ${ }^{46}$ Er verweist auf den "Irrthum der Vorstellungen [...], welche dieses [Geschäft der Philosophie] überhaupt a) in ein Ableiten, es sey aus dem Absoluten oder welchem andern Princip sonst, oder b) in ein Deduciren der wirklichen, erscheinenden Welt, als solcher, oder der Möglichkeit der Erfahrung setzen." ${ }^{47}$

Diese den modernen Deutungsversuchen vorweggenommene Replik Schellings zeigt nur, wie leicht die Explikationen zum Konstruktionsbegriff heute wie damals zu konträren Auslegungen einladen; sonst hätte er kaum zu der Gegendarstellung schon 1802 Anlaß gehabt. Schelling wendet sich gegen die Auffassung, das Verfahren der Konstruktion entweder als Deduktion, bei der aus einem bereits anerkannten Satz als Instanz logische Folgerungen abgeleitet werden, oder als das Erschließen der Bedingungen der Möglichkeit von Erfahrung oder gar als Ableitung von "Einzelgegebenheiten" (Ende) im Sinne einer

39 Boenke (1990) $373 \mathrm{f}$.

40 Krings (1985) $114 \mathrm{f}$.

41 Allgemeine Deduktion des dynamischen Prozesses oder der Kategorien der Physik (1800): SW IV 27.

42 Boenke (1990) 317, 319, 323, 374.

43 Ende (1973) 50.

44 Ende (1973) 54.

45 Ende (1973) 51.

46 System 1802: SW IV 397.

47 System 1802: SW IV 396. 
wirklichen, erscheinenden Welt $\mathrm{zu}$ verstehen. Insbesondere scheint es nicht in der Perspektive dieses Verfahrens zu liegen, es als eine Methode der Beschreibung eines irgendwie genetisch oder generisch strukturierten Bedingungsgefüges zu deuten. Will man das Verfahren schon als "rein logische Konstruktion" (Boenke) oder "spekulative Rekonstruktion" (Krings) umschreiben, so kann damit gewiß nicht der Nachvollzug einer "transzendentalen Genesis" im Sinne des Aufweises konstitutiver Bedingungen objektiver Erkenntnis, wie sie Kant beispielhaft für die Beschreibung naturwissenschaftlicher Erfahrung entwickelt, gemeint sein. Anderenfalls ließe sich nicht einmal der Redeweise nach plausibel machen, worin Schellings Rückgriff auf das Verfahren der Konstruktion im geometrischen Beweis überhaupt seinen Anhalt hat, noch ließe sich der Sache nach aufklären, daß, geschweige denn warum Schelling gerade Kants Begriff der Konstruktion aufnimmt.

Nun spricht Schelling 1798 bei der Einführung des Begriffs von der "Foderung einer ursprünglichen (transscendentalen) Construction"48. 1802 avanciert der Begriff dann zur "absoluten Construktion", die er methodologisch der Demonstration und damit der Beweisführung zuordnet:

"Das, wodurch Construktion absolut ist, ist mit dem, was Princip des Zusammenhangs der philosophischen Demonstration ist, selbst identisch und ein und dasselbe. ${ }^{49}$

Bevor in den folgenden Kapiteln Vollzug, Motive und Ziel der Transformation des Kantischen Konstruktionsbegriffs im einzelnen dargestellt werden sollen, werde ich zunächst einige Anhaltspunkte dafür liefern, daß sich Schelling bei der Beschreibung des Verfahrens wie Kant am euklidischen Beweismodell orientiert, sich jedoch in der philosophischen Interpretation des Verfahrens von Kant unterscheiden muß. Es ist Schelling selbst, der auf die Vorbildfunktion der mathematischen Methodologie hinweist. Die Geometrie gebe "ein allgemeines Beispiel" der von ihm so genannten "absoluten Erkenntnißart":

"Die Geometrie und überhaupt die Mathematik ist bis jetzt die einzige Wissenschaft, welche ein
allgemeines Beispiel jener absoluten Erkenntnißart, die wir auch die demonstrative nennen können,
gegeben hat. Die geometrische Evidenz beruht auf der völligen Aufhebung des Causalgesetzes, sie
erklärt nicht, z.B. wie es komme, daß in einem Dreieck dem größern Winkel immer die größere Seite
gegenüber liege, daß je zwei Seiten zusammengenommen größer seyen als die dritte, sie beweist, daß
es so sey."

Wenn Schelling Konstruktion als demonstrative Erkenntnisart auffaßt, nimmt er zwar, was zunächst überraschen mag, Kants Bestimmung der mathematischen Konstruktion unmittelbar auf, scheint diese Bestimmung jedoch mit der Übertragung in den Bereich philosophischer Demonstration zugleich zu verfehlen. In $\$ 57$ der $K U$ stellt Kant klar:

"Allein aus Gründen a priori kann sie, als Philosophie, zwar beweisen, aber nicht demonstrieren; wenn man nicht ganz und gar von der Wortbedeutung abgehen will, nach welcher demonstrieren [...] so viel heißt, als (es sei in Beweisen oder im Definieren) seinen Begriff zugleich in der Anschauung darstellen: welche, wenn sie Anschauung a priori ist, das Konstruieren desselben heißt, wenn sie aber

48 Übersicht: SS I 451 = AW IV 177.

49 System 1802: SW IV 409.

50 System 1802: SW IV 345. 
auch empirisch ist, gleichwohl die Vorzeigung des Objekts bleibt, durch welche dem Begriff die objektive Realität gesichert wird." ${ }^{51}$

Unter der Ausformulierung des Konstruktionstheorems als Darstellung des Begriffs in der Anschauung a priori versteht Kant eine Form der Demonstration. Kant erläutert die Demonstration in der Perspektive der transzendentalen Theorie der Erfahrung. Danach zielt die Demonstration eines Begriffs auf die Sicherung der objektiven Realität des Begriffs, d.h. den Nachweis, daß der Begriff etwas bezeichnet, dessen Existenz in Raum und Zeit bestimmt werden kann. Läßt sich der Begriff in der Anschauung a priori darstellen, spricht Kant geradezu von der Demonstration als Konstruktion des Begriffs.

Erfolgt die Darstellung des Begriffs "auch empirisch", d.h. auch in der Anschauung a posteriori, spricht Kant zwar nicht mehr von Konstruktion, doch gleichwohl von der Demonstration des Begriffs zum Nachweis seiner objektiven Realität. Damit ist auch die Philosophie der Demonstration fähig, wenn auch nicht "allein aus Gründen a priori"; die Anschauung, in der der Begriff dargestellt wird, muß dann "auch empirisch" sein. Schelling scheint nur Kants These, daß Demonstration, verstanden als Darstellung des Begriffs in der Anschauung a priori, Konstruktion sei, aufnehmen zu wollen. ${ }^{52}$ Die für Kant entscheidende Differenz der Apriorität und Aposteriorität der Darstellung des Begriffs in der Anschauung hat er entweder schlicht übersehen oder so interpretieren können, daß die von ihm vorgenommene Gleichsetzung von Konstruktion und Demonstration - und damit die Überführung des Konstruktionsverfahrens in die philosophische Methodologie - gerade aus der genannten Differenz folgen muß. Die Interpretationsaufgabe dieser Untersuchungen $\mathrm{zu}$ Schelling wird genau darin bestehen, die Theorielage und die Bedingungen zu klären, die den Vollzug der Transformation des Kantischen Konstruktionsbegriffs durch Schelling verständlich werden lassen. Schellings Feststellung:

"Der Demonstration geht die Construktion nicht voran, sondern beides ist eins und unzertrennlich." ${ }^{53}$

macht einerseits, gemessen an Kants Philosophiebegriff, den Vollzug der Transformation manifest, verweist andererseits aber auch auf zeitgenössische Interpretationen des geometrischen Beweisverfahrens, wie sie z.B. Schwab in den "Gedanken über die Analysis" als Einleitung zu seiner Übersetzung von Euklids Data (1780) formuliert:

\footnotetext{
"Insgemein rechnet man zu einer geometrischen Aufgabe weiter nichts als drey wesentliche Stücke; den Satz, der anzeigt, was gegeben ist und was zu thun gefordert wird; die Construction, wodurch der Forderung ein Genüge geschieht, und den Beweis, worin dargethan wird, daß der Forderung durch das Verrichtete wirklich ein Genüge geschehen ist. Der Satz fragt; die Construction antwortet; der Beweis zeigt, daß die Antwort richtig ist: Construction und Beweis werden mit einem gemeinschaftlichen Namen Composition genannt. ${ }^{\text {"N4 }}$
}

Kants Konstruktionstheorem, so habe ich in 4.2. und 4.3. gezeigt, geht aus der transzendentalphilosophischen Interpretation des euklidischen Beweismodells hervor. Wie

$51 K U \S 57$ Anm.I: AA V $241=$ WW V 448f.

52 Vgl. A734 B762: "Selbst das Verfahren der Algeber mit ihren Gleichungen, aus denen sie durch Reduktion die Wahrheit zusamt dem Beweise hervorbringt, ist zwar keine geometrische, aber doch charakteristische Konstruktion, in welcher man an den Zeichen die Begriffe, vornehmlich von dem Verhältnisse der Größen, in der Anschauung darlegt".

53 System 1802: SW IV 407.

54 Schwab (1780) §7. 
sich das nach Proklos in sechs Schritte gegliederte Beweismodell auf die von Schwab genannten "drey wesentliche Stücke" im einzelnen abbilden lassen, führe ich in 9.1. vor. Es wird dann darum gehen, wie Schellings Deutung von Konstruktion als Muster demonstrativer Beweisart vor dem Hintergrund der frühidealistischen Debatte um die Grundlagen der $M A N W$ zu verstehen ist. Hier sei vorläufig auf den besonderen Zusammenhang von Konstruktion und Beweis hingewiesen, hinsichtlich dessen die beiden Schritte als eine Einheit unter dem "gemeinschaftlichen Namen Composition" begriffen werden können. ${ }^{55}$ Damit ist eine Perspektive eröffnet, die die von Hintikka im Sinne Kants rekonstruierte Gliederung des euklidischen Modells in einen synthetischen, eigentlich konstruktiven Teil (ekthesis, kataskeue) und in einen analytischen, eigentlich deduktiven Teil (apodeixis) gänzlich verschiebt. ${ }^{56}$

Soweit aus diesen wenigen Hinweisen deutlich geworden ist, wird man der Komplexität, die Schellings Projekt einer philosophischen Konstruktion mit sich bringt, kaum dadurch gerecht werden, daß man einfachhin ein Überschreiten der Grenzbestimmungen der Vernunft, die Kant in der $K r V$ vorgenommen hat, konstatiert, noch dadurch, daß man Schelling bloß die willkürliche Bildung von Analogien unterstellt. ${ }^{57}$ Schelling entwickelt die These, daß Konstruktion kein Erklären von Sachverhalten im Sinne der Analyse von Entstehungsbedingungen, sondern Beweis ihrer Wahrheit sei, im Kontext seiner FichteKritik des Jahres 1802. Erklären ist Fichte zufolge

"kein Umfassen auf einmal, sondern ein Fortsteigen von einem zum andern, ist etwas endliches, und das Begrenzen, oder Bestimmen ist eben die Brücke, auf welcher übergegangen wird, und die das Ich in sich selbst hat." 58

Fichte selbst hebt hervor, daß ein Erklären in diesem Sinne notwendigerweise zirkulär verlaufe:

"Dies, daß der endliche Geist notwendig etwas absolutes außer sich setzen muß (ein Ding an sich) und dennoch von der anderen Seite anerkennen muß, daß dasselbe nur für ihn da sey [...], ist derjenige Zirkel, den er in das Unendliche erweitern, aus welchem er aber nie herausgehen kann." ${ }^{59}$

Der Sachverhalt, den es durch den Aufweis der Bedingungen seiner Möglichkeit in regressiver Schrittfolge zu erschließen gilt, kann immer nur widersprüchlich als etwas Absolutes, doch nicht anders als in der Perspektive des erkennenden Subjekts beschrieben werden. Was aus der Sicht des Erklärenden "nur für ihn da sey", muß er doch, um es zu bestimmen, als "etwas absolutes außer sich setzen". Das Für-sich-Setzen als einzige Art der

55 Über diesen synthetischen Teil hinaus wird der Beweis durch eine die Konstruktion vorbereitende Analysis des Satzes ergänzt: "Man sieht aber leicht, daß, wenn man über eine Aufgabe selbst nachdenken will, zwischen den Satz und die Construction ein vierter wesentlicher Theil, die Analysis, einzuschieben ist, weil ohne sie [...] die Construction nicht gefunden werden kann." (§7) In $\S 8$ formuliert Schwab die "Allgemeine Regel der Analysis" folgendermaßen: "[...] man erwäge, was man unmittelbar zu finden hat, um das Gesuchte zu finden; hat man es bemerkt, so forsche man ferner nach, wodurch dieses bestimmt werde? und so finde man immer aus dem, was bestimmt werden soll, das Bestimmende, bis man auf den ersten Bestimmungs-Grund [!], das ist, auf das Gegebene oder die Hypothese des Satzes stößt: so ist die Analysis gleicherweise [wie durch das Aufsuchen der Mittelbegriffe] gemacht."

56 Zur Darstellung und Kritik der Rekonstruktion Hintikkas s. 4.2.

57 Vgl. Lukács (1955) 124: "[...] die Methode der 'Konstruktion' des Universums, das heißt die Methode der willkürlichen Zusammenfügung heterogener Phänomene mit Hilfe von bloßen Analogien."

58 Fichte (1794/95) 413.

59 Fichte $(1794 / 95) 412$. 
Vergewisserung des Subjekts muß als ein Außer-sich-Setzen interpretiert werden. Damit bleibt dem Ich unerreichbar, was es sich zu begreifen und zu erklären vornimmt.

Es ist diese Stoßrichtung der Kritik Schellings, die Fichtes Zirkel des Erklärens als einen scheinbaren Widerspruch von Endlichem und Absolutem entlarven will. Dieser Widerspruch gehe auf Fichtes verfehlte Absicht zurück, "welche nicht sowohl das Absolute außer dem Ich, als das Ich außer dem Absoluten halten soll." Schelling jedenfalls kommt zu dem Schluß, "daß jenes außer-sich-Haben des Absoluten (und das freilich mit diesem unmittelbar vergesellschaftete bloße für-sich-Haben, also Gedankenseyn des letzteren) selbst nur ein Schein sey". ${ }^{60}$

Schellings Vorbehalte gegen den von Fichte als notwendig behaupteten Zirkel des Erklärens machen deutlich, daß Konstruktion nicht als ein Verfahren aufgefaßt werden kann, mit dessen Hilfe sich objektkonstitutive Bedingungen in der Perspektive transzendentaler Erkenntnis erschließen lassen. Schelling favorisiert dagegen ein Verfahren, das es erlaube, das Absolute als dem Endlichen immanent zu denken. Diese Immmanenz des Absoluten anhand von "ursprünglichen Schematismen der Weltanschauung" aufzuweisen, nennt er die Funktion philosophischer Konstruktion: Der Philosoph "construirt nicht die Pflanze, nicht das Thier, sondern [...] das Universum in Gestalt der Pflanze, das Universum in Gestalt des Thiers". ${ }^{61}$

Was Schelling mit solchen Formulierungen den Interpreten an Einsicht abverlangt, klingt verwegen genug, um es, noch wohlwollend, vielleicht mit dieser oder ähnlichen Beschreibungen auf sich beruhen zu lassen: Offensichtlich geht es Schelling darum, an der Morphologie biologischer und, so wäre zu ergänzen, anderer naturwissenschaftlicher Systembildungen universelle nomologische Strukturen der Welt aufzuzeigen. Der Ingenieur ist davon überzeugt, daß die auf dem Reißbrett entworfene und durch den Bau realisierte Brückenkonstruktion im Einzelfall denselben mathematisch berechenbaren Gesetzmäßigkeiten unterliegt, die in universalisierter Form in einer allgemeinen physikalischen Theorie aufgestellt sind. Dem Philosophen obliegt es dann, diese abgeleiteten Typen von mathematischen und technischen Konstruktionen auf so etwas wie eine ursprüngliche Konstruktion zurückzuführen, die unser Vertrauen darauf, daß unsere Konstruktionen tatsächlich funktionieren, rechtfertigen und erst verstehen lassen.

Läßt diese Beschreibung das Konzept philosophischer Konstruktion irgendwie erahnen, so muß doch der Gebrauch des Begriffs gerade der Konstruktion als Methodenbegriff der Philosophie, zumal im Anschluß an Kant, als fragwürdig erscheinen. So jedenfalls lautet der Tenor der Schelling-Kritik, die, soweit ich sehe, in der Schelling-Forschung bislang auch nicht überzeugend widerlegt worden ist. Dieser Zustand mag angesichts des spekulativen Niveaus der Texte auch nicht verwundern. Schellings Konzept der philosophischen Konstruktion wirft in der Formulierung von 1802 denn auch erhebliche Verständnisschwierigkeiten auf:

"so ist erstens Construktion überhaupt Darstellung des Besonderen in absoluter Form, philosophische Construktion insbesondere Darstellung des Besonderen in der [...] an sich oder intellektuell 
angeschauten Form. [...] Das andere aber ist die Demonstration selbst, welche Gleichsetzung der Form und des Wesens in solcher Gestalt ist, daß von dem, was in absoluter Form construirt, oder wovon die absolute Idealität erwiesen, unmittelbar auch die absolute Realität erwiesen sey." ${ }^{62}$

Es sind die Rede im Modus der Absolutheit und insbesondere die ungeklärte, in der Forschung noch nicht einmal als Problem formulierte Beziehung von Konstruktion und intellektueller Anschauung ${ }^{63}$, die den direkten hermeneutischen Zugang zu den Texten verstellen, ja nach eigenem Wissenschaftsbegriff verstellen müssen. Denn Schellings Polemik gegen "die Wuth, alles zu erklären" ${ }^{64}$ weist jedes reflektierende Denken nach Grund-Folge-Relationen als negatives Kriterium dessen aus, was er unter absoluter Erkenntnisart und wahrer Wissenschaft verstehen will. Als das "Hauptkriterium" dieser Form von Wissenschaft bezeichnet er denn auch "die gänzliche Abwendung von dem Causalgesetz und derjenigen Welt, in welcher dieses gültig sein kann". ${ }^{65}$ Sofern Konstruktion bei Schelling zu einer "Erkenntniß absoluter Art" avanciert, habe sie "mit der wirklichen Welt als solcher nichts zu schaffen", durch sie werde nicht "die Wirklichkeit, sondern vielmehr nur die absolute Nichtwirklichkeit erkannt". ${ }^{6}{ }^{6}$

Was sich hier als kaum lösbares Problem von Vermittlung und Kritik andeutet, veranlaßt mich, Anspruch und Verfahren einer Interpretation von Schellings Konstruktionsbegriff zu begrenzen. Denn durch den Rückgriff auf so etwas wie die absolute Erkenntnisart, die sich dem begrifflichen Verstehen gerade entzieht, scheint eine hinreichende Explikation dieses Methodenbegriffs von vornherein fraglich, wenn nicht gar unmöglich zu sein.

62 System 1802: SW IV 408.

63 Zum problemgeschichtlichen Kontext der intellektuellen Anschauung bei Schelling s. Wieland (1975) 250-254, Frank (1985) 41-47; zur intellektuellen Anschauung, Konstruktion und Darstellung bei Fichte s. Stolzenberg (1986) 34-60, 118-168.

64 System 1802: SW IV 344.

65 System 1802: SW IV 345.

66 System 1802: SW IV 408f. 


\section{Konstruktion der Begriffe (II): "Gleichsetzung des Begriffs und der Anschauung"}

Im Mittelpunkt steht die Analyse von Schellings Lesart des Kantischen Konstruktionstheorems, soweit sich diese Lesart anhand der durch Höijers Abhandlung über die philosophische Construction vermittelten affirmativen Kant-Kritik Schellings nachvollziehen läßt. Ich beschränke mich dabei auf die detaillierte Beschreibung der Redeweise Schellings und auf die Explikation des Problemhorizontes, vor dem Schelling den Konstruktionsbegriff in der Auseinandersetzung mit Kant entwickelt. Dieses rezeptionsanalytische Verfahren hat den Vorteil, daß Höijers Abhandlung in den Passagen, die sich auf Kant beziehen und die Schelling bei der Abfassung seiner Rezension vor Augen hatte, als Korrektiv herangezogen werden können, um die Motive einzelner Ausdrucks- und Argumentationsweisen bei Schelling aufzuklären. Textgrundlage sind Höijers Abhandlung über die philosophische Construction (1801) und Schellings Rezension dieser Abhandlung, die 1802 unter dem Titel Über die philosophische Construktion $^{1}$ im gemeinsam mit Hegel herausgegebenen Kritischen Journal der Philosophie erschienen ist.

Schelling hatte Höijer auch persönlich kennengelernt, als Höijer, aus Uppsala kommend, im Wintersemester 1798/99 in Jena studierte. ${ }^{2}$ Höijer, der bereits 1796 Fichtes Vorlesungen über die Bestimmung des Gelehrten (1794) ins Schwedische übersetzt hatte, hörte eine Vorlesung Fichtes über Propädeutik der gesamten Philosophie bzw. Logik und Metaphysik. ${ }^{3}$ Höijers Nachschrift ${ }^{4}$ der Vorlesung läßt nicht erkennen, daß er gerade von Fichte zur Abfassung seiner Abhandlung, die Konstruktion als einen philosophischen Methodenbegriff zu etablieren sucht, veranlaßt worden ist. Höijer hat bereits im Frühjahr 1799 das Specimen De Constructione Philosophica ${ }^{5}$ fertiggestellt, also möglicherweise noch während seines Aufenthaltes in Jena begonnen. Das Specimen entspricht einem Teil der weitaus umfangreicheren, 1799 in Stockholm erschienenen Afhandling om den

1 Die Authentizität der Rezension gilt als gesichert. Über den Zuschreibungsstreit berichtet Buchner (1965) 142-152; vgl. den "Editorischen Bericht" in Hegel (1802) 547.

2 Benjamin Carl Henrik Höijer (1767-1812) war von 1789 an Dozent für theoretische und praktische Philosophie, von 1809 an Professor für Philosophie in Uppsala. Zur Biographie s. Gierow (1971) und (1971-1973); zu Profil und Wirkungsgeschichte seiner philosophischen Arbeiten s. Nordin (1987). - Es sei hier nur am Rande darauf hingewiesen, daß in Uppsala 1816-1820 eine Ausgabe Fr.W.J. Schellings sämmtliche Werke erschienen ist. Diese Ausgabe ist der Schelling-Forschung erst vor wenigen Jahren bekannt geworden; Schelling wußte von der Ausgabe. S. die "Editorischen Berichte" in AW II 10-14, 31-33 sowie AW IV 14.

3 Fichte (1794-1812) 34f. legte der Vorlesung Platners Philosophische Aphorismen zugrunde.

4 Höijer (1898): Die Nachschrift liegt im Druck nur als "Excerpt" vor, das zehn Manuskriptseiten zu Fichtes naturphilosophischen Ausführungen unterschlägt. Das vollständige Manuskript umfaßt rund 50 Seiten. Ich danke an dieser Stelle Herrn Dr. Erich Fuchs (Fichte-Kommission, München), der mir eine Kopie des Originals und die vorläufige Übersetzung zur Verfügung gestellt hat.

5 SS V 117-144. 
Philosophiska Constructionen, ämnad til inledning til föreläsningar i philosophien. ${ }^{6}$ Zur Rezension lag Schelling die 1801 wiederum in Stockholm erschienene Übersetzung dieser Abhandlung über die philosophische Construction, als Einleitung zu Vorlesungen in der Philosophie vor. ${ }^{7}$ Nun führt Höijer schon in der "Einleitung" seiner Abhandlung zum Anlaß seines anspruchsvollen Unternehmens aus:

\begin{abstract}
"Übrigens haben wir von Kant selbst die nächste Veranlassung zu der Idee genommen, die wir hier auszuführen suchen. Er construirt in der That die Materie in seinen metaphysischen Anfangsgründen der Naturwissenschaft. Diese Construction ist aber nicht mathematisch; sie geschieht nicht im Raume und in der Zeit, denn durch dieselbe soll jener und mittelbar auch diese erfüllt werden: sie muß also philosophisch seyn." ${ }^{8}$
\end{abstract}

Die Bemerkung Höijers hat dieser Untersuchung die heuristische Perspektive vorgegeben, nach der die Transformation des Konstruktionsbegriffs sich im Rahmen der frühidealistischen Rezeption der $M A N W$ vollzieht. Sie ist darüber hinaus ein Indiz dafür, daß Höijer den Begriff der philosophischen Konstruktion in der Auseinandersetzung mit Schellings Kant-Interpretation ausbildet. Denn auch Schelling hielt im Wintersemester 1798/99 seine ersten naturphilosophischen Vorlesungen nach seinem gleichzeitig bogenweise erscheinenden Ersten Entwurf eines Systems der Naturphilosophie, der Ostern 1799 als Buch vorlag. ${ }^{9}$

Schellings These, wonach Kants Konstruktionstheorem sich als "Gleichsetzung des Begriffs und der Anschauung" verstehen lasse, ist im Hinblick darauf zu untersuchen, welcher Interpretationsansatz dieser Lesart zugrunde liegt. Mit den einleitenden Bemerkungen Schellings lautet die Textpassage:

"Von der Wolfischen Philosophie wollen wir nicht reden, welche in jedem Betracht Dogmatismus ist,
und deren armselige und geistlose Anwendung der äußern Form der geometrischen Methode überdies
keine Idee der Construktion erwecken konnte. Wir wenden uns zu Kant, der die demonstrative
Methode in der Philosophie nur im Geist des Dogmatismus und als logische Analysis begreift, und
der Kritik des Gebrauchs derselben in der Philosophie einen eignen Abschnitt seiner Methodenlehre
gewidmet hat. Was den allgemeinsten Begriff der Construktion betrifft, so ist Kant vielleicht der
erste, der ihn so tief und ächt philosophisch aufgefaßt hat. Er beschreibt Construktion durchgängig
als Gleichsetzung des Begriffs und der Anschauung, und fordert dazu eine nicht-empirische
Anschauung, die einerseits, als Anschauung, einzeln oder concret ist, andererseits als Construktion
eines Begriffs Allgemeingültigkeit für alle möglichen Anschauungen, die unter denselben Begriff
gehören, ausdrücken muß. Ob der dem Allgemeinbegriff Dreieck entsprechende Gegenstand in der
reinen Anschauung oder der empirischen entworfen wird, ist für seine Fähigkeit, den Begriff

6 SS II 1-184. Darüber hinaus gibt es in SS II 185-203 einen fragmentarischen Kommentar (1800) mit dem Titel: Undersökning, huruvida den Philosophi, hvars grundteckningär gifven i Afhandlingen om den Philosophiska Constructionen, bör få namn af idealisk [Untersuchung, inwiefern die Philosophie, deren Grundzüge in der Abhandlung über die philosophische Konstruktion dargelegt sind, idealistisch genannt werden sollte].

7 K.F.A. Schelling teilt im Vorwort (XII) zu SW V mit: "Auch von Höyer ist der Brief da, mit welchem er die Uebersendung seines Buchs an Schelling begleitet und diesen um sein Urtheil bittet, aber kein weiterer." - Auch Reinhold (1803) 208-213 bringt eine Rezension der Abhandlung.

Höijer (1801) 6.

9 Schellings Vorlesungsankündigung für das WS 1798/99 lautet: "Den 18ten October und die zunächstfolgenden Tage, Nachmittags 4. Uhr, werde ich öffentliche Vorlesungen, Über den Begriff und das Wesen der Naturphilosophie, halten. Vom 29ten Oct. an werde ich zu derselben Stunde (von 4-5), wöchentlich 4mal das System der Naturphilosophie selbst nach meinem Entwurf desselben, wovon in Kurzem [...] die ersten Bogen erscheinen werden, vortragen." Im Lektionsverzeichnis werden abweichend "philosophiam naturae" und "Idealismi transcendentalis initia" angekündigt. (BuD I 169, vgl. 163) 
unbeschadet seiner Allgemeinheit auszudrücken, ganz gleichgültig, weil bei der empirischen Anschauung selbst doch nur auf die Handlung der Construktion des Begriffes an und für sich selbst gesehen wird u.s.w." ${ }^{10}$

Schellings Feststellung, daß Kant "Construktion durchgängig als Gleichsetzung des Begriffs und der Anschauung" 'beschreibe', muß zunächst überraschen. Soweit ich sehe, ist der Ausdruck "Gleichsetzung" in diesem Zusammenhang weder in der $K r V$ noch im gesamten Kantischen Werk belegt. Kant selbst spricht regelmäßig von Konstruktion als Darstellung des Begriffs in der Anschauung. Nun soll der Ausdruck Gleichsetzung "den allgemeinsten Begriff der Construktion" bezeichnen, den - wie Schelling hervorhebt - Kant "so tief und ächt philosophisch aufgefaßt" habe. Als Beleg führt er die Textpassage A713f. B742f. an, in der Kant am Beispiel der geometrischen Darstellbarkeit des Allgemeinbegriffs Dreieck die wesentlichen Momente seines Konstruktionstheorems erläutert. ${ }^{11}$ Im Hinblick auf diese Stelle und seine Deutung von Konstruktion als Gleichsetzung sieht sich Schelling in Übereinstimmung mit Kant. Er glaubt sogar, den Leser im Anschluß an seine teils wörtliche, teils paraphrasierende Wiedergabe der Stelle mit der Abkürzung "u.s.w." zur Erläuterung des Sachverhalts ohne weiteres an den Wortlaut der $K r V$ verweisen zu können. So lautet denn auch abschließend sein zustimmender Kommentar: "Bis hierher drückt Kant die Idee der Construktion und den Grund aller Evidenz vollkommen aus." ${ }^{12}$

Dieser affirmative Teil der Kant-Kritik Schellings gibt nun ersten Aufschluß über die Art, wie Schelling die Textvorlage liest und versteht. Schellings Anliegen ist es ja, Konstruktion im Rückgang auf Kants Interpretation des geometrischen Konstruktionsbegriffs als Methode in der Philosophie zu etablieren, dies jedoch offensichtlich gegen Kants eigene Absicht. Wenn Schelling nun annimmt, Kant beschreibe (auch) an der zitierten Stelle der Idee nach "den allgemeinsten Begriff der Construktion", so ist davon auszugehen, daß er Kants Bestimmung mathematischer Erkenntnisart von vornherein so liest, als ob Kant der Sache nach die Anwendung der Konstruktion in der Philosophie uneingestanden bereits zuläßt oder zulassen müßte. Ein Blick in die Rezensionsvorlage zeigt, daß Schelling und Höijer im heuristischen Ansatz ihrer Deutung übereinstimmen. Darauf deutet ein Vergleich jeweils der Einleitungs- und Schlußphrasen ihrer 'Interpretation'13 der Stelle:

"Nach der allgemeinsten und ausgedehntesten Bedeutung von Construction [...]. - Bisher sind wir mit Kant in vollkommener Übereinstimmung." ${ }^{14}$

"Was den allgemeinsten Begriff der Construktion betrifft [...]. Bis hierher drückt Kant die Idee der Construktion [...] vollkommen aus." ${ }^{15}$

Der Formulierung Schellings, wonach Kant Konstruktion als Gleichsetzung 'beschreibe', läßt sich noch ein anderer als bloß unspezifischer Sinn geben. Denn aufhorchen läßt ja, daß

10 Konstruktion: SW V $127 \mathrm{f}$.

11 Vgl. die Synopse der Textpassagen in 8.1.

12 Konstruktion: SW V 128.

13 Höijer (1801) 55: "Was wir mit seinen Lehrsätzen vorgenommen, besteht darin, daß wir sie vielleicht genauer bestimmt, und ihn also nur interpretirt haben."

14 Höijer (1801) 51, 55.

15 Konstruktion: SW V $127 \mathrm{f}$. 
Kant das, was Schelling hier 'Beschreibung' nennt, im Kontext der Differenzierung von philosophischer und mathematischer Erkenntnisart als Nominaldefinition einführt, wobei Konstruktion genaugenommen als negatives Unterscheidungskriterium der Erkenntnisarten dient:

"Die philosophische Erkenntnis ist die Vernunfterkenntnis aus Begriffen, die mathematische aus der Konstruktion der Begriffe. Einen Begriff aber konstruieren, heißt: die ihm korrespondierende Anschauung a priori darstellen." (A713 B741)

Beide Arten der Vernunfterkenntnis sind auf Begriffe bezogen, unterscheiden sich aber im Gebrauch der Begriffe. Es hat den Anschein, als ob Kant die philosophische Erkenntnis als Erkenntnis aus Begriffen zunächst gar nicht erläutert. Dagegen macht er das Kennzeichen der mathematischen Erkenntnis, die Konstruktion der Begriffe, zum unterscheidenden Merkmal beider Arten der Vernunfterkenntnis. So spricht Kant rückblickend in den MARL davon, daß das Konstruktionstheorem "die Definition der Philosophie überhaupt angeht, welche der Verfasser der Kritik d. r. V. für sein eigenes, nicht unerhebliches Produkt ausgibt". Dabei bezeichne seine Ausformulierung als "Darstellung eines gegebenen Begriffs in der Anschauung a priori" genau das Kriterium, "wodurch auf einmal die Philosophie von der Mathematik ganz bestimmt geschieden wird."16

Faßt man in diesem Sinn Konstruktion als negatives Merkmal der Definition philosophischer Erkenntnis auf, so läßt sich Kants Bestimmung der Erkenntnisarten als Nominaldefinition $^{17}$, näher als "verneinende Definition" philosophischer Erkenntnis klassifizieren. Dabei gibt das Merkmal der Konstruktion zwar den Unterscheidungsgrund an, ist jedoch "zur Erkenntnis der Sache ihrer inneren Möglichkeit nach" nicht hinreichend. ${ }^{18}$ Der Sinn dieser Differenzierung wird klarer, wenn man die Kriterien, die Kant für die Gewinnung von Erkenntnis formuliert, auf die Theorie, in der sie formuliert werden, selber anwendet. Die Kantische Definition der Differenz der Erkenntnisarten weist darauf hin, daß eine Realdefinition der Philosophie selber gar nicht möglich ist, weil sie aufweisen müßte, daß und wie sie möglich sei. Zwar vermag sie das Kriterium zu bestimmen, durch dessen Erfüllung der Nachweis gelänge, daß ihren Begriffen "objektive Realität, d.i. Bedeutung und Wahrheit"19 ${ }^{19}$ zukommt, nämlich durch die Darstellung der Begriffe in der Anschauung. Doch kann dieser Nachweis nur durch die Ausführung des Programms der transzendentalen Begründung von Erfahrung als naturwissenschaftlicher Erkenntnis gegeben werden. Kants Deutung des geometrischen Konstruktionsbegriffs erhellt nun gerade aus der Funktion, die der Mathematik in diesem Programm zukommt. ${ }^{20}$

16 MARL: AA VI 207f. = WW IV 312.

17 L §106: AA IX 143 = WW III 575: "Unter bloßen Namen-Erklärungen oder Nominal-Definitionen sind diejenigen zu verstehen, welche die Bedeutung enthalten, die man willkürlich einem gewissen Namen hat geben wollen, und die daher nur das logische Wesen ihres Gegenstandes bezeichnen, oder bloß zu Unterscheidung desselben von andern Objekten dienen. - Sach-Erklärungen oder Real-Definitionen hingegen sind solche, die zur Erkenntnis des Objekts, seinen innern Bestimmungen nach, zureichen, indem sie die Möglichkeit des Gegenstandes aus innern Merkmalen darlegen."

18 L 106: AA IX 144 = WW III 576: "Bloß verneinende Definitionen können auch keine Real-Definitionen heißen, weil verneinende Merkmale wohl zur Unterscheidung einer Sache von andern eben so gut dienen können, als bejahende, aber nicht zur Erkenntnis der Sache ihrer innern Möglichkeit nach."

$19 M A N W$ : AA IV $478=$ WW V 23.

$20 \mathrm{Vgl}$. 4.3. 
Der Nachweis, daß und wie Mathematik auf Objekte der Erfahrung angewandt werden kann, vermag zugleich die "Begriffe und Lehrsätze [...] (eigentlich der Transzendentalphilosophie) zu realisieren, d.i. einer bloßen Gedankenform Sinn und Bedeutung unterzulegen." ${ }^{21}$ In diesem Sinne ist die Transzendentalphilosophie einer Selbstdefinition und damit auch einer Definition ihrer Fundamentalbegriffe gar nicht fähig, da sie zu deren Umsetzung stets auf das Bezug nehmen muß, was sie zu begründen vorgibt. Sie würde also zirkulär verfahren, indem sie die Begriffe, die sie zu definieren unternimmt, immer schon voraussetzen muß. ${ }^{22}$

Es liegt in der Konsequenz des transzendentalen Konzepts von Erkenntnis, daß die Bestimmung der Erkenntnisarten in der $\mathrm{KrV}$ als einer philosophischen Disziplin nur "zum bloßen Versuche" (A730 B759) oder, so man will, als "Annäherung" zur Definition und nur zu "Expositionen" taugt (A731* B759*). Kant formuliert später tatsächlich einmal:

"Die negative Definition der Transsc. Ph. ist: daß sie ein Princip synthetischer Erkentnis a priori aus Begriffen ist wodurch sie zwar von der Mathematik unterschieden aber nicht begreiflich wird, wie eine solche Philosophie als diejenige die transscendental heißt möglich ist." ${ }^{23}$

Diese Bemerkung Kants erinnert daran, daß er unter philosophischer Erkenntnis keine Begriffsanalyse verstehen will. Bereits in der $K r V$ weist er darauf hin, daß die eigentümliche Differenz von philosophischer und mathematischer Erkenntnisart erst in der Perspektive der "vorgetragenen transzendentalen Grundlagen" erhelle: "Es kommt hier nicht auf analytische Sätze an, die durch bloße Zergliederung der Begriffe erzeugt werden können [...], sondern auf synthetische, und zwar solche, die a priori sollen erkannt werden." (A718 B746) Grundlage der Unterscheidung beider Erkenntnisarten ist demnach die Klasse synthetischer Sätze a priori, wobei gelten soll, daß die Erkenntnisarten sich gerade nicht in der "Allgemeinheit der Erkenntnis und ihrer Erzeugung a priori" unterscheiden, jedoch - wie es in A723 B751 heißt - "im Fortgange sehr verschieden" sind.

Wie ich in 4.3. dargelegt habe, läßt sich der hier nur metaphorisch als "Fortgang" beschriebene Sachverhalt als der Unterschied im Nachweis der objektiven Realität transzendentaler und mathematischer Begriffe bestimmen. Genaugenommen geht es um die unterschiedlichen Funktionen und Anteile, die (transzendental)philosophische und mathematische Erkenntnisart am Nachweis der objektiven Realität empirischer Erkenntnis haben. Kurzgefaßt besagt dieses Konzept, daß sowohl transzendentale wie mathematische Begriffe in der Anschauung 'dargestellt' werden können. (A720f. B748f.) Die Differenz zeigt sich in der Aposteriorität bzw. Apriorität der Darstellung. Die Begriffe drücken eine 'Synthesis möglicher Anschauungen' aus, die nach den konstitutiven (mathematischen) Grundsätzen des Verstandes a priori in der reinen Anschauung oder nach den regulativen (dynamischen) Grundsätzen a posteriori in der empirischen Anschauung gegeben werden. Dieses Datum ist die 'Bedingung'24 jeder mathematischen Konstruktion und ihrer

21 MANW: AA IV $278=$ WW V 24.

22 Kant hat die Definition der Kategorien bewußt unterlassen (A82 B108) und die Erklärung der Fundamentalprinzipien der Apperzeption und des Schematismus (A141 B181f.) für unmöglich gehalten.

23 OP: AA XXI 94. Vgl. Büchel (1987) 45.

${ }^{24} M A N W$ : AA IV 486f. = WW V 34. 
Anwendung auf 'besondere Naturdinge', d.h. der Mathematisierung dessen, was wir als Objekt der Erfahrung nach physikalischen Kriterien beschreiben können.

Die Charakterisierung der Erkenntnisarten als intuitiv ('in der Anschauung') und diskursiv ('durch Begriffe') ist das Ergebnis der transzendental genannten Reflexion auf die als Erkenntnis interpretierte 'Eine Erfahrung', deren konstitutive und regulative Momente nach den erwähnten Grundsätzen des Verstandes isoliert darstellbar sind:

"Das Erkenntnis durch Begriffe heißt diskursiv, das in der Anschauung intuitiv; in der Tat wird zu einer Erkenntnis beides mit einander verbunden erfordert, sie wird aber von dem benannt, worauf, als den Bestimmungsgrund desselben, ich jedesmal vorzüglich attendiere." 25

Unter dem Bestimmungsgrund ist die Bedingung bzw. das Datum jeder Darstellung von Begriffen (als Erfahrungserkenntnis) zu verstehen. Erfolgt die Bestimmung aufgrund der in dem Begriff ausgedrückten Synthesis reiner Anschauungen (nach Gestalt, Dauer, Zahl) ${ }^{26}$, so spricht Kant von der Darstellung a priori des Begriffs in der Anschauung oder von Konstruktion:

\footnotetext{
"Den mathematischen Begriff eines Triangels würde ich konstruieren, d.i. a priori in der Anschauung geben, und auf diesem Wege eine synthetische, aber rationale Erkenntnis bekommen. Aber, wenn mir der transzendentale Begriff einer Realität, Substanz, Kraft usw. gegeben ist, so bezeichnet er weder eine empirische, noch reine Anschauung, sondern lediglich die Synthesis der empirischen Anschauungen (die also a priori nicht gegeben werden können), und es kann also aus ihm, weil die Synthesis nicht a priori zu der Anschauung, die ihm korrespondiert, hinausgehen kann, auch kein bestimmender synthetischer Satz, sondern nur ein Grundsatz der Synthesis möglicher empirischer Anschauungen entspringen. Also ist ein transzendentaler Satz ein synthetisches Vernunfterkenntnis nach bloßen Begriffen, und mithin diskursiv". (A722 B750)
}

Erfolgt die Bestimmung aufgrund der in dem Begriff ausgedrückten Synthesis empirischer Anschauungen, so spricht Kant von der Darstellung a posteriori des Begriffs in der Anschauung:

\footnotetext{
"Demnach lassen sich transzendentale Sätze niemals durch Konstruktion der Begriffe, sondern nur nach Begriffen a priori geben. Sie enthalten bloß die Regel, nach der eine gewisse synthetische Einheit desjenigen, was nicht a priori anschaulich vorgestellt werden kann, (der Wahrnehmungen,) empirisch gesucht werden soll. Sie können aber keinen einzigen ihrer Begriffe a priori in irgendeinem Falle darstellen, sondern tun dieses nur a posteriori, vermittelst der Erfahrung, die nach jenen synthetischen Grundsätzen allererst möglich wird." (A720f. B748f.)
}

Die Disjunktion von Begriff und Anschauung ist das Ergebnis einer methodischen Isolierung, die das, was wir als Faktum der Erfahrung vorfinden, nach einem Kanon von Regeln systematisch auf die Bedingungen hin erschließt, die es uns erst möglich machen, von Erfahrung als Erkenntnis im Sinne wahrheitsdifferenter Urteile zu sprechen. Als transzendentale Terme sind Begriff und Anschauung als Momente der Dekomposition der 'Einen Erfahrung' begreifbar, deren spezifische Funktion, wissenschaftssystematisch gesehen, nach dem Konzept der Vermittlung von Mathematik und Naturwissenschaft durch eine metaphysische Theorie der Erfahrung bestimmt wird. Analog zur

$25 F$ : AA XX $325=$ WW III 666.

26 "Aber im Raume eine Anschauung a priori zu bestimmen (Gestalt), die Zeit zu teilen (Dauer), oder bloß das Allgemeine der Synthesis von einem und demselben in der Zeit und dem Raume, und die daraus entspringende Größe einer Anschauung überhaupt (Zahl) zu erkennen, das ist ein Vernunftgeschäft durch Konstruktion der Begriffe, und heißt mathematisch." (A724 B752) 
Dekomposition der 'Einen Erfahrung' nach den intuitiven und diskursiven Weisen ihrer Repräsentation durch die Sinnlichkeit und Konstitution durch den Verstand ist es die dem Konstruktionstheorem immanente Voraussetzung der 'Korrespondenz' von Begriff und Anschauung, die Schelling einen ersten Anhaltspunkt bieten wird, die Formel 'Darstellung des Begriffs in der Anschauung' als 'Gleichsetzung des Begriffs und der Anschauung' zu deuten. Während Kant die Aufgabe und Leistung von Philosophie nicht in Konkurrenz zu Naturwissenschaft und Mathematik, sondern als deren Komplement sieht, hat Schelling das Bild einer Wissenschaftshierarchie vor Augen, die der Philosophie den obersten Rang zuweist. Schelling wird denn auch im Gegenzug zur Kantischen Konzeption vom "Selbstbeweis" der Philosophie sprechen. ${ }^{27}$

Wenn Schelling nun Kants Definitionsversuch als 'Beschreibung' versteht, so wird er damit nicht auf die Unzulänglichkeit und mangelnde Präzision der Definition anspielen wollen. ${ }^{28}$ Anderenfalls hätte er kaum hervorgehoben, daß Kant hiermit "den allgemeinsten Begriff der Construktion [...] so tief und ächt philosophisch aufgefaßt" habe. Zieht man Höijers Abhandlung hinzu, läßt sich der Ausdruck "beschreiben" folgendermaßen präzisieren:

"Eine Definition [...] enthält die Beschreibung einer Construction, die ich mit ihrer Hilfe zugleich begreife und verrichte." 29

"Desgleichen sind auch bestimmte und richtige Definitionen in der Philosophie nicht möglich, wenn sie nicht die Entstehung und Bildung der Begriffe beschreiben, welches nichts anderes ist, als deren Construction selbst darstellen." 30

Was eine Definition ausmacht, ist hier offenbar die "Beschreibung einer Construction". Sie gibt eine Anleitung ("Hilfe"), die das Verstehen des Begriffs als dessen Realisierung deutet. Höijer macht die Korrektheit philosophischer Definitionen davon abhängig, daß sie "die Entstehung und Bildung der Begriffe beschreiben", soll heißen, "deren Construction selbst darstellen." Dies deckt sich mit der Ausgangsbestimmung, wonach eine Definition "die Beschreibung einer Construction" sei. Daraus folgt aber, daß sich Beschreibung genau als "Entstehung und Bildung der Begriffe", mithin selbst schon als Konstruktion verstehen läßt. Dieser Sachverhalt liegt auch Höijers Bemerkung zugrunde, daß ich mit Hilfe der Beschreibung die Konstruktion "zugleich begreife und verrichte".

Höijers Erörterungen zum Definitionsbegriff nutzen die Konvergenz geometrischer und transzendentaler Semantik aus; näher betrachtet, verweisen sie auf einen eigentümlichen Zusammenhang von Mathematik- und Urteilstheorie, an dem die Wahl der Terminologie ihren Anhalt auch in der Sache hat. Höijer selbst verweist auf Kants Ausführungen zum Vergleich geometrischer und transzendentaler Terme in B154. Dort erläutert Kant die

27 System 1802: SW IV 371.

28 Vgl. L \$105 über Erörterungen und Beschreibungen: "Nicht alle Begriffe können also, sie dürfen aber auch nicht alle definiert werden. Es gibt Annäherungen zur Definition gewisser Begriffe; dieses sind teils Erörterungen (expositiones), teils Beschreibungen (descriptiones). [...] Die Beschreibung ist die Exposition eines Begriffs, so fern sie nicht präzis ist." (AA IX 142f. = WW III 574)

29 Höijer (1801) 60. "verrichte" verbessert aus "vernichte"; vgl. schwedische Version, SS II 62f.: "Definition [...] innehaller en beskrifning på en construction, hvilken jag med dess tilhjelp på en gång både begriper och verkställer."

30 Höijer (1801) 61; vgl. SS II 63: "[...] om de ej beskrifva begrepens upkomst och formerande, som är at visa sjelfva deras construction." 
Wirkungsweise der 'transzendentalen Handlung der Einbildungskraft' unter dem Stichwort der 'figürlichen Synthesis' als etwas, das "wir auch jederzeit in uns wahr[nehmen]. Wir können uns keine Linie denken, ohne sie in Gedanken zu ziehen, keinen Zirkel denken, ohne ihn zu beschreiben". (B154) Dieser 'reine Aktus' - Kant spricht von einer "Handlung des Subjekts" - bezeichne eine "Bewegung, als Beschreibung eines Raumes [...] und gehört nicht allein zur Geometrie, sondern sogar zur Transzendentalphilosophie." (B155*) ${ }^{31}$

Was Höijer genauer als 'Bildung' der Begriffe umschreibt, gibt nun einen impliziten Hinweis auf den mathematiktheoretischen Hintergrund. Bildung ist die Übersetzung des schwedischen "formerande", was soviel wie "Formgebung", "Gestaltung", "Erzeugung" bedeutet. ${ }^{32}$ In seiner lateinischen Version als formatio wird dieser Begriff in zwei Bedeutungen gebraucht, die für unsere Fragestellung wichtig werden: (a) In der Theorie der Geometrie wird mit formatio gerade der Schritt der Konstruktion bezeichnet. Kant ist diese Bedeutung vertraut; er erwähnt sie in der Vorrede zu MARL, und zwar im Zusammenhang der Rechtfertigung seiner in $\operatorname{der}$ ä $\mathrm{KrV}$ formulierten "Definition der Philosophie überhaupt". ${ }^{33}$ (b) In der Urteilstheorie ist die Wendung "formamus judicia" geläufig. ${ }^{34}$ Schelling spricht etwa von der "Formation eines Urteils". ${ }^{35}$ Höijer verweist einmal auf "alle Abstraction, wodurch ein allgemeiner Begriff eines Dinges formirt wird". ${ }^{36}$ Im folgenden versuche ich $\mathrm{zu}$ zeigen, daß beide Konnotationen des Begriffs der formatio terminologisch wie systematisch für den transzendentalphilosophischen Kontext, in dem Kant des Konstruktionstheorem entwickelt, von Bedeutung sind.

(a) In der "Vorrede" der MARL verwahrt sich Kant gegen den Vorwurf des Plagiats, was seine in der $K r V$ formulierte "Definition der Philosophie überhaupt angeht". ${ }^{37}$ Als vermeintliche Quelle hatte "ein Tübingscher Rezensent" eine Erläuterung des Mathematikers C.A. Hausens ausfindig gemacht. ${ }^{38}$ Kant zitiert die Textstelle vollständig:

"Porro de actuali constructione hic non quaeritur, cum ne possint quidem sensibiles figurae ad rigorem definitionum effingi; sed requiritur cognitio eorum, quibus absolvitur formatio, quae intellectualis quaedam constructio est. C.A. Hausen, Elem. Mathes. Pars I. p. 86. A. 1743." ${ }^{39}$

\footnotetext{
Vgl. 5.6.

ordbok över svenska språket 18 (1926) 1148-1151: "formera".

MARL: AA VI 207f. = WW IV 312f.

Z.B. Koethen (1737) 51. Nach AW I 174 kannte Schelling dieses Buch seit 1792.

System 1800: SW III 509.

Höijer (1801) 34; vgl. SS II 36: "all abstraction, hvarigenom et allmänt begrep om något ting formeras".

MARL: AA VI $207=$ WW IV 312.

38 Vermutlich J.Fr. Flatt in den Tübingenschen gelehrten Anzeigen (1795) 815. In der Rezension einer in Erlangen erschienenen mathematischen Dissertation von J.C. Yelin heißt es: "In Ansehung der Construction dieser und anderer krummer Linien erinnert d. H. Vf. noch mit Recht, daß dadurch nicht nothwendig eine mechanische Beschreibung verstanden werde, und diese also keiner praktischen Richtigkeit bedürfe; weil nun heut zu Tag alles in der Kantischen Sprache ausgedrückt sein muß, so nennt er die Construction einer Größe eine Darstellung durch reine Anschauung." Zit. nach AA VI 521.

39 MARL: AA VI 208 = WW IV 312. - Weischedels Übersetzung: "Ferner wird hier nicht nach der wirklichen Darstellung gefragt, da die sinnlich wahrnehmbaren Figuren ja nicht nach der Strenge der Definitionen gebildet werden können; sondern gesucht wird eine Erkenntnis dessen, wodurch diejenige Gestaltung zustandekommt, die eine gleichsam durch den Verstand gemachte Darstellung ist."
} 
formatio bezeichnet nun dasjenige, was der "intellectualis quaedam constructio" entspricht. Kant kommentiert die Stelle folgendermaßen:

"Die gleichsam durch den Verstand gemachte Darstellung bedeutete dem Mathematiker nichts
weiter, als die einem Begriffe korrespondierende (empirische) Verzeichnung einer Linie, bei der bloß
auf die Regel Acht gegeben, von den in der Ausführung unvermeidlichen Abweichungen aber
abstrahiert wird; wie man in der Geometrie auch an der Konstruktion der Gleichungen wahrnehmen
kann."

Kant paraphrasiert hier offensichtlich die Stelle A713f. B741f., auf deren urteilstheoretischen Aussagegehalt nun näher einzugehen ist:

\begin{abstract}
"Einen Begriff aber konstruieren, heißt: die ihm korrespondierende Anschauung a priori darstellen. Zur Konstruktion eines Begriffs wird also eine nicht empirische Anschauung erfordert, die folglich, als Anschauung, ein einzelnes Objekt ist, aber nichtsdestoweniger, als die Konstruktion des Begriffs (einer allgemeinen Vorstellung), Allgemeingültigkeit für alle möglichen Vorstellungen, die unter denselben Begriff gehören, in der Vorstellung ausdrücken muß. So konstruiere ich einen Triangel, indem ich den diesem Begriffe entsprechenden Gegenstand, entweder durch bloße Einbildung, in der reinen, oder nach derselben auch auf dem Papier, in der empirischen Anschauung, beidemal aber völlig a priori, ohne das Muster dazu aus irgendeiner Erfahrung geborgt zu haben, darstelle. Die einzelne hingezeichnete Figur ist empirisch, und dient gleichwohl den Begriff, unbeschadet seiner Allgemeinheit, auszudrücken, weil bei dieser empirischen Anschauung immer nur auf die Handlung der Konstruktion des Begriffs, welchem viele Bestimmungen, z.E. der Größe, der Seiten und der Winkel, ganz gleichgültig sind, gesehen, und also von den Verschiedenheiten, die den Begriff des Triangels nicht verändern, abstrahiert wird."
\end{abstract}

Kants Bestimmung der mathematischen Erkenntnis als Konstruktion der Begriffe besagt, daß die Korrespondenz von Begriff und Anschauung durch eine Darstellung a priori realisiert werden muß. Dieses Darstellungsverfahren geht von dem Bild einer Spannung zwischen Begriff und Anschauung aus, deren Aufbau und Überwindung die Argumentation in A713f. B741f. strukturiert. Diese Spannung äußert sich auch in der doppelten Funktion, der der Rekurs auf die nichtempirische Anschauung zur Konstruktion des Begriffs dient: Als Anschauung sei sie ein einzelnes Objekt und zugleich Ausdruck der Allgemeingültigkeit für alle möglichen Anschauungen, die unter den Begriff gehören. Für die Allgemeingültigkeit steht die Anschauung offenbar nicht allein kraft der Nichtempirizität (Reinheit) ein, sondern kraft des Konstruktions- bzw. Darstellungsverfahrens des Begriffs, dadurch daß sie auf den durch den Begriff bezeichneten Gegenstand bezogen wird, in dessen Vorstellung die Anschauung die Allgemeingültigkeit 'ausdrücken' muß. Von Bedeutung ist daher nicht nur die Abstraktion von den veränderlichen Bestimmungen dessen, was durch den Begriff bezeichnet wird, sondern auch die Handlung der Konstruktion, deren Apriorität unabhängig davon feststeht, ob ich in der reinen Anschauung das Dreieck konstruiere bzw. in der empirischen Anschauung die Figur hinzeichne. Ich rekurriere dabei lediglich auf die bloße Einbildung in der reinen oder nach derselben auch in der empirischen Anschauung, um den dem Begriff entsprechenden Gegenstand darzustellen.

Soweit aus dieser Textparaphrase hervorgeht, läßt sich Kants Konzept von Begriffen überhaupt durch zwei Momente charakterisieren: Allgemeinheit und Allgemeingültigkeit. $\mathrm{Da}$ der Begriff eine allgemeine Vorstellung ist, also Allgemeinheit hat, besagt dabei, daß

40 MARL: AA VI $208=$ WW IV $312 \mathrm{f}$. 
unter den Begriff diejenigen Vorstellungen zu subsumieren sind, deren gemeinsames Merkmal der Begriff repräsentiert. Die Allgemeinheit des Begriffs kann daher nicht als Komplexion von Merkmalen, sondern muß als Abstraktionsallgemeinheit aufgefaßt werden. ${ }^{41}$

\footnotetext{
"Nun muß man zwar einen jeden Begriff als eine Vorstellung denken, die in einer unendlichen Menge von verschiedenen möglichen Vorstellungen (als ihr gemeinschaftliches Merkmal) enthalten ist, mithin diese unter sich enthält; aber kein Begriff, als ein solcher, kann so gedacht werden, als ob er eine unendliche Menge von Vorstellungen in sich enthielte." (B39f.)
}

Wird von den Verschiedenheiten der durch den Allgemeinbegriff bezeichneten Objekte abstrahiert, so bezeichnet diese Abstraktionsallgemeinheit genauer "die logische Form eines Begriffs (des Denkens) überhaupt" (A237 B298), wonach der Umfang des Begriffs als unendliche Menge unbestimmter, doch bestimmbarer logischer Objekte konzipiert ist. Von der Allgemeinheit des Begriffs ist zu unterscheiden die Allgemeingültigkeit, die anders als die formallogische die transzendentallogische Funktion des Begriffs, d.h. die Beziehung von Begriff und Gegenstand, bestimmt.

"Zu jedem Begriff wird erstlich die logische Form eines Begriffs (des Denkens) überhaupt, und dann zweitens auch die Möglichkeit, ihm einen Gegenstand zu geben, darauf er sich beziehe, erfordert. Ohne diesen letzteren hat er keinen Sinn, und ist völlig leer an Inhalt, ob er gleich noch immer die logische Funktion enthalten mag, aus etwaigen datis einen Begriff zu machen." (A239 B298)

Nach Kants Formulierung soll das Konstruktion der Begriffe genannte Verfahren nun darin bestehen, daß dabei von den variablen Bestimmungen der durch den Begriff bezeichneten Objekte abstrahiert wird und, unbeschadet der Allgemeinheit des Begriffs, die einzelne Anschauung, auf die sich der Begriff bezieht, die Allgemeingültigkeit ausdrückt. Bei der Konstruktion kommt es offenbar nicht darauf an, daß ich eine bestimmte geometrische Figur hinzeichne, sondern allein darauf, daß auch diese hingezeichnete Figur die Allgemeingültigkeit des konstruierten Begriffs ausdrückt.

Nun beschreibt Kant in A713f. B741f. die mathematische Erkenntnisart in ihrer eigentümlichen Differenz zur philosophischen Erkenntnisart, und er erläutert sie, wie angedeutet, in der Terminologie der transzendentalen Logik. Nach der in 4.3. vorgelegten Interpretation des Konstruktionstheorems besteht die der transzendentalen Erfahrung immanente Differenz in dem direkten (konstitutiven) und indirekten (regulativen) Rekurs auf das Datum der Darstellung des Begriffs in der reinen oder empirischen Anschauung. Sieht man auf das transzendentale Moment der 'Form' empirischer Erkenntnis, so wird der als Erscheinung interpretierte Fall empirischer Erkenntnis in der reinen sinnlichen Anschauung hinsichtlich der 'Gestalt', 'Dauer' und 'Zahl' durch die mathematische Erkenntnisart bestimmt. (A724 B758) Dieser formale 'Bestimmungsgrund' liefert nun das Datum der Darstellung a priori in genau der "Anschauung, [...] in welcher dasjenige, was aus den allgemeinen Bedingungen der Konstruktion folgt, auch von dem Objekte des konstruierten Begriffs allgemein gelten [!] muß." (A715f. B743f.) Die in der Anschauung ausgedrückte Allgemeingültigkeit des Begriffs ist deswegen auf eine unendliche Menge von bestimmbaren Objekten übertragbar, weil sie sich auf die (nach mathematischer

$41 \mathrm{Zu}$ Extension und Intension eines Begriffs in Kants Theorie der allgemeinen und transzendentalen Logik vgl. Schulthess (1981) 104-121. 
Erkenntnisart bestimmbaren und eigentlich quantifizierbaren) formalen Elemente der empirischen Erkenntnis beziehen.

(b) Höijers Ausführungen zum Konstruktionsbegriff enthielten bereits einen Hinweis auf die Konvergenz von mathematischer und transzendentallogischer Terminologie. In (a) sollten die urteilstheoretischen Implikationen des Konstruktionstheorems, soweit sie sich mit der in 4.3. vorgelegten Deutung verbinden ließen, sichtbar gemacht werden. Im folgenden und in 9.3. versuche ich zu zeigen, daß Schelling das Theorem zwar auch im Kontext einer Urteilstheorie gelesen hat, die mit der transzendentalen Theorie Kants jedoch nur insofern kompatibel ist, als sie deren noch fehlende Prämissen zu liefern verspricht. Es ist bezeichnend, daß Schellings Rede von der "Formation eines Urtheils" gerade im Zusammenhang mit der Beschreibung eines Urteilsaktes über einen geometrischen Gegenstand steht:

"Daß ich, sowie ich einen Triangel erblicke, er sey nun von welcher Art er wolle, in demselben Augenblick das Urtheil fälle, diese Figur sey ein Triangel, setzt eine Anschauung von einem Triangel überhaupt, der weder stumpf- noch spitz- noch rechtwinklich ist, voraus, und wäre vermöge eines bloßen Begriffs vom Triangel so wenig als vermöge eines bloßen Bilds von demselben möglich; denn da das letztere nothwendig ein bestimmtes ist, so wäre die Congruenz des wirklichen mit dem bloß eingebildeten Triangel, wenn sie auch wäre, eine bloß zufällige, welches zur Formation eines Urtheils nicht zulänglich ist." ${ }^{42}$

Schelling geht es um die Aufklärung der Frage nach der "Congruenz" von wirklichem und eingebildetem Gegenstand, also der Frage nach dem Grund der Beziehung von Gegenstand und der Vorstellung des Gegenstandes. ${ }^{43}$ Diese Beziehung wird als Urteil gedeutet, mit dem die 'Übereinstimmung' von Vorstellung und Gegenstand behauptet wird. Diese Beziehung könnte dann nicht als notwendig und wahr gelten, wenn bei der "Formation des Urtheils" jeweils nur von einem 'bloßen Begriff' oder 'bloßen Bild' als Relaten in Urteilen auszugehen wäre. Schelling kommt es hier darauf an, die Rolle des 'Schemas', "das die Vermittlung beider macht", zu bestimmen. Aufhorchen läßt nun die Sprachregelung, mit der er den Urteilsakt beschreibt: "im Urtheil wird eine Anschauung einem Begriff gleich gesetzt". ${ }^{4}$

Wenn Schelling das Kantische Konstruktionstheorem als "Gleichsetzung des Begriffs und der Anschauung" deutet, so scheint er das Theorem geradezu als Beschreibung eines Urteils aufzufassen. Die Rede von der Gleichsetzung läßt vermuten, daß im Urteil etwas, das nicht gleich oder ungleich ist, verglichen und dann gleich gesetzt wird. Tatsächlich bestimmt Schelling Urteilen als Vergleichen: "im Urteil wird [...] verglichen", und zwar "nicht etwa Begriff mit Begriff, sondern es werden Begriffe mit Anschauungen verglichen." ${ }^{45}$ Die Momente des Begriffs und der Anschauung bilden die Subjekt-PrädikatStruktur des Urteils ab und ermöglichen zugleich die Reflexion auf die Glieder der im Urteil vollzogenen Gleichsetzung:

42 System 1800: SW III 509.

43 Zum Vorgehen, wie Schelling die schon von Kant im Herz-Brief vom 21.2.1772 formulierte Fragestellung interpretiert, vgl. 6.3.

44 System 1800: SW III 511.

45 System 1800: SW III $507 \mathrm{f}$. 
"Das Prädikat ist an sich vom Subjekt nicht verschieden, denn es wird ja, eben im Urtheil, eine Identität beider gesetzt. Also ist die Trennung von Subjekt und Prädikat überhaupt nur dadurch möglich, daß jenes die Anschauung, dieses den Begriff repräsentiert." ${ }^{46}$

Dabei versteht Schelling den "Begriff überhaupt" durchaus im transzendentallogischen Sinn als "etwas, das Vielheit in Einheit zusammenfaßt". ${ }^{47}$ Diesen Synthesis-Charakter grenzt er dann auch gegen die formallogische Bestimmung ab: "Also ist der Begriff nicht, wie insgemein vorgegeben wird, das Allgemeine, sondern vielmehr die Regel, das Einschränkende, das Bestimmende der Anschauung". ${ }^{48}$ Nach Schelling ist der Begriff dann "die Regel, nach welcher das Objekt überhaupt construirt wird, das Objekt dagegen nicht die Regel, sondern der Ausdruck der Regel selbst". ${ }^{49}$

Schelling gibt hier eine urteilslogische Beschreibung des Kantischen Konstruktionstheorems. Der Begriff formuliert die Konstruktionsregel, nach der die Anschauung bestimmt und so das Objekt "entsteht" oder "construirt wird"50. Übereinstimmend mit Schelling sollte auch bei Kant die Anschauung, verstanden als "einzelnes Objekt" und als "die einzelne hingezeichnete Figur", den Begriff bzw. die Allgemeingültigkeit des Begriffs "ausdrücken". (A713f. B741f.) Im Unterschied zu Schelling wird jedoch nicht das Objekt, sondern der Begriff des Objekts konstruiert. Kant spricht davon, daß in der Anschauung, die den Begriff darstellt, "dasjenige, was aus den allgemeinen Bedingungen der Konstruktion folgt, auch von dem Objekte des konstruierten Begriffs allgemein gelten muß." (A715f. B743f.) Kant unterscheidet also von der Konstruktion des Begriffs noch "allgemeine Bedingungen der Konstruktion", die von der Konstruktionsregel, die der Begriff vorgibt, verschieden sind und gleichwohl von dem Objekt des konstruierten Begriffs allgemein gelten sollen. Wie in (a) bereits dargelegt, bezieht sich die Allgemeingültigkeit des konstruierten Begriffs auf die formalen, nach mathematischer Erkenntnisart bestimmbaren und eigentlich quantifizierbaren Elemente der 'Gestalt', 'Dauer' und 'Zahl' empirischer Erkenntnis. Als eine solche Bedingung der Konstruktion nennt Kant das Datum oder den Bestimmungsgrund, die nach der transzendentallogischen Interpretation des euklidischen Beweisverfahrens den Begriff in der reinen Anschauung a priori oder in der empirischen Anschauung a posteriori darstellen. Die apriorische Darstellung erfolgt konstitutiv nach den 'mathematischen' Grundsätzen des Verstandes, die aposteriorische Darstellung erfolgt regulativ nach den 'dynamischen' Grundsätzen. Als transzendentale Grundsätze regeln sie die Anwendung quantifizierender Meßverfahren auf etwas, das Dasein hat und für uns Gegenstand möglicher Erfahrung ist.

Kant vermag so das mathematische Konstruktionsverfahren in seine philosophische Theorie der Erfahrung zu integrieren. Daher kann er auch auf den Bestand solcher "allgemeinen Bedingungen" der Konstruktion der euklidischen Geometrie zurückgreifen. Dieser Bestand der reinen Mathematik beschreibt einen Teilbereich der "Wirklichkeit der wissenschaftlichen Erkenntnisse a priori" (B128), deren Anwendung in der

46 System 1800: SW III 508.

47 Vom Ich: SW I 184 = AW II 109.

48 System 1800: SW III 512.

49 System 1800: SW III 508.

50 System 1800: SW III 508. 
Naturwissenschaft es durch die tranzendentale Theorie der Erfahrung zu verstehen und zu begründen gilt.

Schellings urteilslogische Beschreibung des Konstruktionstheorems weist auf ein weitergehendes Verständnis des Kantischen Programms hin. Begriff und Anschauung werden als Momente eines Reflexionsaktes aufgefaßt, durch den die Glieder der Gleichsetzung repräsentiert und benannt werden. Als Glieder markieren sie jedoch schon die Trennung und Aufhebung der ursprünglichen Identität dessen, was vom Standpunkt der Reflexion die Subjekt-Prädikat-Struktur eines Urteils ausmacht. Begriff und Anschauung repräsentieren nur die Struktur des Urteils. Konstitutiv für den Urteilsakt ist Schelling zufolge so etwas wie der ursprüngliche Schematismus: "Denn im Urtheil wird eine Anschauung einem Begriff gleich gesetzt; damit dieß geschehe, muß etwas seyn, was die Vermittlung beider macht, und dieß ist allein das Schema." ${ }^{51}$

Schelling beschreibt den Schematismus näher als Handlung, "in welcher die Regel selbst das Objekt, oder in welcher umgekehrt das Objekt als Regel der Construktion überhaupt angeschaut würde." Daß Schelling hier im Irrealis formuliert, deutet darauf hin, daß die das Urteil konstituierende Handlung zwar als Konstruktion verstanden, doch selbst nicht anders als urteilslogisch und so nur unzulänglich beschrieben, aber nicht erklärt werden kann. Daher fährt er fort: "Eine solche Anschauung ist der Schematismus, welchen jeder nur aus eigner innerer Erfahrung kennen lernen, und den man, um ihn kenntlich zu machen und die Erfahrung zu leiten, nur beschreiben [...] kann. ${ }^{52}$

Kants Absicht war es, durch die philosophische Interpretation des euklidischen Beweismodells das Konstruktionsverfahren in die philosophische Theorie selber zu integrieren, um auf diese Weise die 'Geometrisierung der Natur' innerhalb der Naturwissenschaften zu verstehen und zu begründen. Schelling geht über diese Absicht hinaus, indem er auf der Grundlage der Kantischen Interpretation den Konstruktionsbegriff zur Beschreibung und Aufklärung der fundamentalen Begriffe und Prinzipien der Transzendentalphilosophie selbst anwendet. Er wird von der "Selbstkonstruktion" des Prinzips der Transzendentalphilosophie sprechen. ${ }^{53}$

Vor dem Hintergrund dieser Untersuchungen zur Terminologie, in der Kants Konstruktionstheorem reformuliert wird, läßt sich nun auch der Ansatz der KantInterpretation Schellings verständlicher machen. Wiederum gibt Höijer einen Anhaltspunkt:

"Was die Philosophen für die Entdeckung der Wahrheit bisher ausgerichtet, haben sie keiner logischen Analyse, sondern bloß der Construction ihrer Begriffe zu danken, die sie unwissentlich vorgenommen haben. [...] wie sind seine [Kants] Expositionen alsdann auch nur möglich?" ${ }^{54}$

Nach der Maxime, "Kant construire, ohne es zu wissen" (Höijer) oder Kant "müßte construiren" (Schelling), kann man unterstellen, daß Kants Distinktion so aufgefaßt wurde, 
als habe er, wenn auch "unwissentlich", tatsächlich konstruiert. ${ }^{55}$ Diese von Höijer wie Schelling vertretene These beruht auf zwei Annahmen: Kant beschränke die Funktion philosophischer Erkenntnis auf die logische Analyse von Begriffen, während er das intuitive Moment, d.h. den Bezug auf eine reine Anschaungsmannigfaltigkeit, der Mathematik vorbehalte. Einerseits postuliere er zwischen beiden Disziplinen einen "absoluten Unterschied", orientiere aber an der Bestimmung mathematischer Erkenntnis die Norm von Erkenntnis überhaupt, die seine Definition philosophischer Erkenntnis eben nicht erfüllen könne: "Da Kant für die Geometrie die nicht-empirische Anschauung zugibt, so kann er den absoluten Unterschied zwischen Mathematik und Philosophie nicht darein setzen, daß es für diese eine nicht-empirische Anschauung geben müßte, dergleichen es doch nicht gebe." ${ }^{56}$ Der hier exponierte Ansatz der durch Höijer vermittelten affirmativen Kant-Kritik Schellings ist durch eine Detailanalyse der Reformulierung des Konstruktionstheorems zu erläutern.

55 Vgl. Höijer (1801) 45: "Hat denn Kant selbst nicht philosophirt?" - Schelling merkt an, "daß der Philosoph nach Kant mit den Begriffen, auf die er beschränkt ist, nicht anders als analytisch zu Werk gehen könne. Ist denn das Kants Meynung, oder hat dieses spätere Kapitel ["Methodenlehre" der $\mathrm{KrV}$ ] die frühern vergessen?" (Konstruktion: SW V 133)

56 Konstruktion: SW V 129. 


\section{Die Transformation des Konstruktionsbegriffs (1796/97)}

Die Lesart, die Schelling dem Konstruktionstheorem in der Rezension (1802) von Höijers Abhandlung über die philosophische Construction gibt, ist bereits das Ergebnis der Transformation des Kantischen Konstruktionsbegriffs. Ausgehend von Beobachtungen zu Schellings Rezeption der kritizistischen Terminologie 1795-1803, soll das Motiv der Transformation erschlossen und der Vollzug in seinen einzelnen Schritten beschrieben werden.

\subsection{Ein Textvergleich: KrV: A713f. B741f. - Konstruktion: SW V 127f.}

Schelling erläutert seine These, daß Kant den Begriff der Konstruktion als "Gleichsetzung des Begriffs und der Anschauung" beschreibe, durch Verweis auf die Stelle KrV A713f. B741f., die er teils wörtlich wiedergibt, teils paraphrasiert. Anhand der Konfrontation beider Passagen sollen zunächst Übereinstimmungen und Unterschiede festgestellt werden. Der Vergleich macht deutlich, wie sich die bereits vollzogene Transformation des Konstruktionsbegriffs in Schellings Reformulierung des Kantischen Konstruktionstheorems ausdrückt.

Kant

$K r V:$ A713f. B741f.

(a) "Einen Begriff aber konstruieren, heißt: die ihm korrespondierende Anschauung a priori darstellen.

(b) Zur Konstruktion eines Begriffs wird also eine nicht empirische Anschauung erfordert,

(c) die folglich, als Anschauung, ein einzelnes Objekt ist,

(d) aber nichtsdestoweniger, als die Konstruktion eines Begriffs (einer allgemeinen Vorstellung), Allgemeingültigkeit für alle möglichen Anschauungen, die unter denselben Begriff gehören, in der Vorstellung ausdrücken muß.
Schelling

Konstruktion: SW V 127f.

"Er beschreibt Construktion durchgängig als Gleichsetzung des Begriffs und der Anschauung,

und fordert dazu eine nicht-empirische Anschauung,

die einerseits, als Anschauung, einzeln oder concret ist,

andererseits als Construktion eines Begriffs Allgemeingültigkeit für alle möglichen Anschauungen, die unter den Begriff gehören, ausdrücken muß. 
(e) So konstruiere ich einen Triangel,

(f) indem ich den diesem Begriffe entsprechenden Gegenstand, entweder durch bloße Einbildung, in der reinen, oder nach der derselben auch auf dem Papier, in der empirischen Anschauung, beidemal aber völlig a priori ohne das Muster dazu aus irgendeiner Erfahrung geborgt zu haben, darstelle.

(g) Die einzelne hingezeichnete Figur ist empirisch,

(h) und dient gleichwohl den Begriff, unbeschadet seiner Allgemeinheit, auszudrücken,

(i) weil bei dieser empirischen Anschauung immer nur auf die Handlung der Konstruktion des Begriffs,

Ob der dem Allgemeinbegriff Dreieck entsprechende Gegenstand in der reinen Anschauung oder der empirischen entworfen wird,

\begin{abstract}
ist für seine Fähigkeit, den Begriff unbeschadet seiner Allgemeinheit auszudrücken, ganz gleichgültig,

weil bei der empirischen Anschauung selbst doch nur auf die Handlung der Construktion des Begriffes
\end{abstract}

an und für sich selbst gesehen wird u.s.w."

Schelling gibt den Wortlaut des Kantischen Textes nur verkürzt wieder. Es fehlt die Erläuterung des Konstruktionstheorems durch das Beispiel der geometrischen Konstruktion eines Dreiecks (e,g,j,). Die beiden Sätze, in denen Kant auf die Ausführung der geometrischen Konstruktion (e,f) und die Eigenschaften der dann "hingezeichneten Figur" (g-k) eingeht, faßt Schelling zu einem Satz zusammen. Schellings Wiedergabe verzichtet auf die exemplifikatorischen Elemente der Vorlage, wodurch der Akzent auf die begriffstheoretischen Bestimmungen des Theorems gelegt wird. Dementsprechend geht er auch nicht von "diesem Begriffe" eines Dreiecks aus, das "durch bloße Einbildung" oder "auf dem Papier" konstruiert werde, sondern von "dem Allgemeinbegriff Dreieck" (f). Schelling legt es offenbar auf eine abstraktere und verallgemeinernde Darstellung des Sachverhalts an.

Während Schelling einzelne Formulierungen exakt übernimmt und so z.B. an der Unterscheidung von "Allgemeinheit" und "Allgemeingültigkeit" eines Begriffs überhaupt festhält, scheint er einige markante Ausdrücke bewußt zu umgehen: 'a priori darstellen' (a,f), 'Objekt' (c), 'Vorstellung' (d). Die Veränderungen, die Schelling an Kants Text vorgenommen hat, sind jedoch nicht beliebig, sondern lassen sich vom Ansatz seiner KantInterpretation her motivieren und verstehen. 
Die Passage gehört zum affirmativen Teil seiner Kant-Kritik, nach der Kant ja "den allgemeinsten Begriff der Construktion" beschreibe. Diese Behauptung läßt den Schluß zu, daß aus der Explikation des Konstruktionstheorems in Schellings Paraphrase sich bereits die Übertragung des Konstruktionsbegriffs auf die Philosophie als gerechtfertigt erweisen lassen müßte. Verschärfend muß sogar gesagt werden, daß die Kantische Formulierung des Theorems in den Augen Schellings schon so etwas wie eine philosophische Konstruktion ausdrückt. Schelling liest Kant so, als ob Kant konstruiert oder wenigstens konstruieren müßte.

Mithin verfügt Schelling über eine Lesart der Stelle A713f. B741f., die ihn gerade da, wo er sich mit Kant einig glaubt, zu einer Kant entgegengesetzten Schlußfolgerung kommen läßt. Diesem hier exponierten Interpretationsansatz folgt im übrigen auch Höijer. Zwar gibt er zu bedenken: "Vielleicht befaßt aber die Bedeutung, die Kant dem Worte Construction giebt, etwas, das zwar auf die Mathematik, nicht aber auf die Philosophie paßt?" ${ }^{1}$ Doch leitet er aus seiner Auffassung, daß Kant uneingestanden doch konstruiere ${ }^{2}$, den Vorsatz ab, den Begriff der Konstruktion "so zu bestimmen, daß er sowohl bey unserm Begriff der Anschauung, als bey dem seynigen, paßt, und bey den Folgen von beyden gilt."

Schelling wie Höijer sitzen keiner naiven Fehlinterpretation des Kantischen Konstruktionstheorems auf. Beide halten ihre Lesart mit der Theorie Kants für kompatibel, obgleich beide dem Anschein nach zu einem dem Kantischen entgegengesetzten Ergebnis kommen. Sie sind sich der, wie sie meinen, bloß scheinbaren Differenz zu Kant durchaus bewußt, die verschwände, wenn - so der Ansatz- und Revisionspunkt der frühidealistischen Kant-Kritik - die noch fehlenden Prämissen der Resultatgestalt der Kantischen Philosophie rekonstruiert worden seien. Die im Vergleich der Textpassagen festgestellten Unterschiede im Ausdruck bieten erste Anhaltspunkte, Ausgang und Ziel der Transformation des Konstruktionsbegriffs vor dem Hintergrund der frühen Kant-Kritik Schellings näher zu bestimmen. In einer Fußnote zu §6 der Abhandlung Vom Ich (1795) präzisiert Schelling die Bedeutung von vier Schlüsselbegriffen der Kantischen Philosophie:

"Das Wort empirisch wird gewöhnlich in einem gar zu eingeschränkten Sinne genommen. [...] Rein ist, was ohne allen Bezug auf Objecte gilt. Erfahrungsmäßig, was nur durch Objecte möglich wird. A priori, was nur in Bezug auf Objecte (nicht durch sie) möglich ist. - Empirisch das, wodurch Objecte möglich sind."

Im Vergleich mit Kants Verwendung der Begriffe sind diese Bestimmungen auf den ersten Blick ungewöhnlich. Es fällt auf, daß die Begriffe durchgängig in ihrer Beziehung auf Objekte bestimmt werden. Schelling korreliert offenbar die Begriffe jeweils in Zweiergruppen nach ihrem entgegengesetzten Objektbezug. Folgende Paare lassen sich sinnvoll zuordnen: 


\begin{tabular}{|c|c|c|}
\hline \multirow[t]{2}{*}{ (Ia) } & rein & - ohne allen Bezug auf Objekte \\
\hline & a priori & - nur in Bezug auf Objecte \\
\hline \multirow[t]{2}{*}{ (Ib) } & erfahrungsmäßig & - durch Objecte möglich \\
\hline & empirisch & - wodurch Objecte möglich \\
\hline \multirow[t]{2}{*}{ (IIa) } & rein & - ohne allen Bezug auf Objecte \\
\hline & empirisch & - wodurch Objecte möglich \\
\hline \multirow[t]{2}{*}{ (IIb) } & a priori & - nur in Bezug auf Objecte \\
\hline & erfahrungsmäßig & - durch Objecte möglich \\
\hline
\end{tabular}

Kombination (IIa) und (IIb) entspricht jeweils der Kantischen Dichotomie, auf die Schelling auch anspielt, wenn er 'a priori' durch den adversativen Zusatz in Klammern "was nur in Bezug auf Objecte (nicht durch sie) möglich ist" von 'erfahrungsmäßig' unterscheiden will. Allerdings leuchtet die Entgegensetzung (IIb) nicht ein, da 'erfahrungsmäßig' auch als ein Fall von 'a priori' aufgefaßt werden kann. Die Bedeutung des Ausdrucks "in Bezug auf Objecte" schließt die des Ausdrucks "durch Objecte möglich" nicht aus, sondern spezifiziert sie nur. Schon nach der parallelen Formulierung der Paarbildungen (Ia) und (Ib) ist zu schließen, daß Schelling an etwas anderem als der bloßen Wiedergabe der Kantischen Einteilung gelegen ist. Wie die einleitende Bemerkung der Fußnote deutlich macht, geht es Schelling auch darum, auf Konnotationen des Empirie- bzw. Erfahrungsbegriffs hinzuweisen; das zeigt die auch bei Schelling ungewöhnliche Übersetzung von 'a posteriori' als 'erfahrungsmäßig'. ${ }^{5}$

Selbst wenn man den Kontext, in dem Schelling diese Begriffe entwickelt, noch vernachlässigt, so lassen sich aus diesen Bestimmungen für das Ausgangszitat dieses Abschnitts durchaus schon Erklärungsansätze gewinnen. Zur Konstruktion oder Darstellung a priori des Begriffs, so heißt es, sei eine nicht empirische (b), genauer eine reine Anschauung (f) erforderlich. Da Schelling die reine Anschauung jedoch als "ohne allen Bezug auf Objecte" bestimmen würde, geriete er in Widerspruch, wenn er wie Kant eben diese Anschauung als "ein einzelnes Objekt" (c) kennzeichnete. Tatsächlich charakterisiert er diese Anschauung denn auch lediglich als "einzeln oder concret". Entsprechend umgeht Schelling auch die Übernahme des Ausdrucks 'a priori darstellen', da nach seiner Festlegung der Ausdruck 'a priori' etwas bezeichnet, was "nur in Bezug auf Objecte" ausgesagt wird. Im folgenden soll der Kontext erläutert werden, in dem Schelling

5 Schelling verwendet die Paarbildung 'a priori' - 'a posteriori' in : SW I $94=$ AW I 276, SW I $424=$ AW IV 151, SW I 427 = AW IV 154, SW I 471f. = AW IV 189 u.ö. - In SW I 25 = AW I 86 erläutert er 'a priori' als Gegensatz zu 'experientia' so: "h.e. secundum principia ex ipsa animi humani indole hausta". 
die Begriffsbestimmungen entwickelt. Anzusetzen ist bei seiner Kritik des Empiriebegriffs, die ihm bei der Suche nach den Prämissen der Transzendentalphilosophie Kants eine Perspektive eröffnet hat, das Verfahren der philosophischen Konstruktion einzuführen.

\section{2. "Empirisch ist das, wodurch Objecte möglich sind." (1795)}

Schelling spricht in $\S 6$ der Abhandlung Vom Ich davon, daß das Wort empirisch "gewöhnlich in einem gar zu eingeschränkten Sinne genommen" werde, und führt dazu aus:

"Empirisch ist alles, was dem reinen Ich entgegengesezt ist, also überhaupt im Bezug auf ein NichtIch steht, selbst das Ursprüngliche, im Ich selbst, gegründete, Entgegensezen eines Nicht-Ichs, durch welche Handlung dieses überall erst möglich wird. [...] Empirisch [ist] das, wodurch Objecte möglich sind."6

Die Begriffsnot, die - wie Schelling bekennt - seinen Untersuchungen "eine ganz eigne Unfaßlichkeit"7 beschert, läßt sich leicht auch an dem zitierten Text ausmachen. Die gewöhnlich zu enge Bedeutung des Wortes 'empirisch' ersetzt Schelling durch eine Bestimmung, die "alles, was dem reinen Ich entgegengesezt ist, also überhaupt im Bezug auf ein Nicht-Ich steht", bezeichnen soll. Dabei ist der mit "also" eingeleitete Teilsatz nicht als Explikation, sondern als Folgerung zu verstehen. Denn der Bezug von allem Empirischen auf ein Nicht-Ich folgt aus seiner Entgegensetzung gegen das reine Ich. Dieser Bezug besteht aber nicht in der Entgegensetzung gegen das reine Ich. Anderenfalls stünde das reine Ich, indem ihm etwas entgegengesetzt ist, in Bezug auf ein Nicht-Ich. Es wäre damit selbst empirisch-bedingt und bezeichnete etwas, "wodurch Objecte möglich sind", und widerspräche damit seiner Auszeichnung als etwas, "was ohne allen Bezug auf Objecte gilt." Demnach muß daraus, daß etwas dem reinen Ich entgegengesetzt ist, folgen, daß es in Bezug auf ein Nicht-Ich steht, ohne daß das reine Ich durch diese Entgegensetzung selbst auf das Nicht-Ich bezogen würde und so empirisch-bedingt wäre. Das reine Ich scheint vielmehr eine Voraussetzung dafür zu bezeichnen, daß Empirisches, das Objekte möglich macht, gedacht werden kann.

Schelling beschreibt mit dieser Denkfigur den "Widerstreit zwischen dem reinen und empirisch-bedingten Ich". Mit der Auflösung dieses Widerstreits könne gezeigt werden, "daß Philosophie selbst möglich werde". ${ }^{8}$ Schelling fordert daher ein Prinzip, das zwar Empirizität und somit Objekte zu denken möglich macht, nicht jedoch durch diese Beziehung auf Objekte wiederum selbst empirisch-bedingt sei. Er nennt es im Unterschied zum endlichen oder empirisch-bedingten Ich das unendliche Ich:

"Der Charakter der Endlichkeit ist, nichts sezen zu können, ohne zugleich entgegenzusezen. Diese Form der Entgegensezung ist ursprünglich bestimmt durch die Entgegensezung des Nicht-Ichs. Es ist nemlich dem Ich nothwendig, indem es sich selbst absolut gleich sezt, zugleich alles Nicht-Ich sich entgegenzusezen, was nicht möglich ist, ohne das Nicht-Ich selbst zu sezen. Das unendliche Ich 
würde alles Entgegengesezte ausschliessen, ohne es sich entgegenzusezen: es würde überhaupt alles sich schlechthin gleich sezen, also, wo es sezt, nichts als seine Realität sezen".'

$\mathrm{Daß}$ Schelling den Schlußsatz dieser Passage in den Irrealis setzt, zeigt, daß er sich über die Schwierigkeiten der sprachlichen Vermittlung dieser Prinzipienspekulation durchaus im klaren ist. Diese Schwierigkeiten treten immer dann auf, "sobald wir das, was niemals Object werden kann, zum logischen Object machen." ${ }^{10}$ Und "insofern wir mit unserm Erkennen an Objecte gebunden sind"11, müssen wir uns auf dieser objektsprachlichen Ebene bewegen. Daher bestimmt Schelling das unendliche Ich negativ als dasjenige, "was für sich selbst schlechterdings nicht Object, und für etwas ausser ihm weder Object noch Nichtobject, d.h. gar nichts seyn kann"12. Es ist nun zu zeigen, daß Schelling diese mit Bezug auf den Objektbegriff gegebene Bestimmung des Prinzips der Philosophie in der kritischen Auseinandersetzung mit Kants Konzeption eines höchsten Prinzips der Transzendentalphilosophie entwickelt.

Schellings Absicht, durch Lösung jenes "Widerstreits zwischen dem reinen und empirischbedingten Ich" den Nachweis zu führen, "daß Philosophie selbst möglich werde"13, kündigt er in der Vorrede seiner Abhandlung Vom Ich so an: "Ich habe versucht, die Resultate der kritischen Philosophie in ihrer Zurückführung auf die lezten Principien alles Wissens darzustellen." ${ }^{14}$ Damit formuliert Schelling eine Aufgabe, deren Antwort er im Titel der Abhandlung bereits vorwegnimmt: Vom Ich als Princip der Philosophie oder über das Unbedingte im menschlichen Wissen. Schelling nennt dieses Prinzip das unendliche oder auch das "absolute, alles Entgegengesezte ausschließende Ich": "Dieses als das Einige Unbedingbare bedingt die ganze Kette des Wissens, beschreibt die Sphäre alles Denkbaren, und herrscht durch das ganze System unsers Wissens als die absolute alles begreifende Realität." ${ }^{15}$ Den Nachweis der Möglichkeit der Philosophie verknüpft Schelling mit der Klärung der Frage, wie sich Objektivität oder die Möglichkeit, Objekte zu denken, erklären lasse. Selbst unbedingbar, muß das Ich doch als Bedingung der Entgegensetzung des empirischen Ich und des Nicht-Ich gedacht werden. Im Unterschied zum reinen Ich kündige sich das empirische Ich "nicht durch das blosse: Ich bin; sondern durch das: Ich denke, an, d.h. es ist nicht durch sein blosses Seyn, sondern dadurch, daß es Etwas, daß es Objecte denkt."16

Was Schelling 'empirisch' nennt, bezeichnet zugleich einen konstitutiven Bestandteil von Objektivität: "Empirisch [ist] das, wodurch Objecte möglich sind." ${ }^{17}$ Als solches begreift er nun das, was Kant als den höchsten Punkt der Transzendentalphilosophie ausmacht: "die synthetische Einheit der Apperzeption [ist] der höchste Punkt, an dem man allen Verstandesgebrauch, selbst die ganze Logik, und, nach ihr, die Transzendental-Philosophie

\footnotetext{
Vom Ich §8: SW I 180* = AW II 104*.

Vom Ich 3 : AW II 91 = SW I 168.

Ebd.

Vom Ich §7: SW I $177=$ AW II 101.

Vom Ich: SW I 176 = AW II 100.

Vom Ich: SW I 152 = AW II $70 f$.

Vom Ich: SW I $176=$ AW II 100.

Vom Ich: SW I 180* = AW II 105*.

Vom Ich §6: SW I 176* = AW II 100*.
} 
heften muß, ja dieses Vermögen ist der Verstand selbst." (B134*) Daß Schelling gerade die ursprünglich-synthetische Einheit der Apperzeption, "Das: Ich denke" (B131), als etwas ausweist, das "Objecte denkt" und aufgrund dieser Beziehung auf Objekte als empirischbedingt bezeichnet, hat seinen Anhalt genau an der Funktion, die Kant von der Apperzeption als der "objektiven Einheit des Selbstbewußtseins" (B139) sprechen läßt. In dieser Funktion ist sie nicht nur "eine objektive Bedingung aller Erkenntnis", sondern auch "Bedingung alles Denkens" (B138). Nun hat Kant diese Einheit dreifach charakterisiert:

(a) "Ich nenne sie die reine Apperzeption, um sie von der empirischen zu unterscheiden" (B132). Die empirische Einheit bezeichnet die Einheit des Bewußtseins "in dem, was empirisch ist", und "ist in Ansehung dessen, was gegeben ist, nicht notwendig und allgemein geltend." (B140) Kant verdeutlicht die "nur subjektive Gültigkeit" dieser Einheit an einem Beispiel: "Einer verbindet die Vorstellung eines gewissen Wortes mit einer Sache, der andere mit einer anderen Sache" (B140). Sofern sich die empirische Einheit auf "empirische Bedingungen" bezieht und "selbst eine Erscheinung betrifft", ist sie von der reinen Apperzeption als der Funktion, die solche Erscheinungen gleichwohl als Gegenstände der Erfahrung zu bestimmen möglich macht, nur "abgeleitet". (B140)

(b) Kants zweite Bestimmung des Prinzips lautet: "Ich nenne sie [...] auch die ursprüngliche Apperzeption, weil sie dasjenige Selbstbewußtsein ist, was, indem es die Vorstellung Ich denke hervorbringt, die alle anderen muß begleiten können, und in allem Bewußtsein ein und dasselbe ist, von keiner weiter begleitet werden kann." (B132) Das Merkmal, ursprünglich zu sein, heißt, von keinem höheren Prinzip abgeleitet zu sein, und zeichnet die Apperzeption selbst als höchstes Prinzip aus. Man hat hier von einem Einheits- und Identitätsprinzip gesprochen. ${ }^{18}$ Als einfach und doch auf Mannigfaltiges bezogen, beschreibt es zugleich die durchgängige Identität des Ich in dieser Beziehung auf alle Vorstellungen, die es sich als die seinen zuschreibt.

(c) Kant gibt der Apperzeption noch eine dritte Bestimmung: "Ich nenne auch die Einheit derselben die transzendentale Einheit des Selbstbewußtseins, um die Möglichkeit der Erkenntnis a priori aus ihr zu bezeichnen." (B132) "Das: Ich denke" ist selbst eine Vorstellung, die, als solche "hervorgebracht", einen "Aktus der Spontaneität" bezeichnet (B132); genaugenommen ist diese Vorstellung ein "Aktus der Spontaneität der Vorstellungskraft", die man im Unterschied zur Sinnlichkeit oder Vorstellungsfähigkeit "Verstand nennen muß". (B130)

Sofern nun der Verstand, "allgemein zu reden, das Vermögen der Erkenntnisse" (B137) ist, und Erkenntnisse "in der bestimmten [d.h. unter der Einheit der Apperzeption kategorial vermittelten] Beziehung gegebener Vorstellungen auf ein Objekt" bestehen (B137), ist die Einheit der Apperzeption bei Kant in der Tat stets objektbezogen definiert.

"Die synthetische Einheit des Bewußtseins ist also eine objektive Bedingung aller Erkenntnis, nicht deren ich bloß selbst bedarf, um ein Objekt zu erkennen, sondern unter der jede Anschauung stehen muß, um für mich Objekt zu werden". (B138)

18 Henrich (1976) 56, 58; Cramer (1986) 56-63. 
Kant beschreibt den Sachverhalt, daß etwas als auf Objekte bezogen gedacht werden kann, unter zwei Hinsichten: erstens ist das vorstellende Subjekt, "ich" genannt, es selbst, das der Einheit bedarf, "um ein Objekt zu erkennen"; zweitens ist eine Anschauung erst dann dazu qualifiziert, "für mich Objekt zu werden", wenn sie unter der Einheit steht. Diese von Kant "auch [...] die transzendentale Einheit des Selbstbewußtseins" (B132) genannte "Einheit der Apperzeption" (B138) bezeichnet nur einen, den dritten Gesichtspunkt, unter dem sich "der höchste Punkt, an dem man [...] die Transzendental-Philosophie heften muß" (B134), bestimmen läßt. Es gilt, "die Möglichkeit der Erkenntnis a priori aus ihr zu bezeichnen." (B132) Die Funktion dieses eigentlich transzendentalen Gesichtspunktes nimmt jedoch die durch die Merkmale des Reinen und Ursprünglichen beschriebenen Funktionen der Apperzeption bereits in Anspruch. So definiert sich das Merkmal der Apperzeption, rein zu sein (a), in Abgrenzung gegen die empirische Einheit des Bewußtseins, gegen deren "nur subjektive Gültigkeit" Kant die reine Einheit als "allein objektiv gültig" (B140) profiliert. Und wenn Kant die Apperzeption "auch die ursprüngliche" nennt, "weil" sie - wie in (b) angeführt - als Einheits- und Identitätsprinzip aufzufassen ist (B132), so will er auch "diese ursprüngliche Verbindung" (B133) immer schon auf solche Vorstellungen bezogen wissen, die "nicht insgesamt meine Vorstellungen sein [würden], wenn sie nicht insgesamt zu einem Selbstbewußtsein gehörten" (B132). Daß ich mir Vorstellungen als meine Vorstellungen - selbst dann, wenn "ich mich ihrer [...] nicht als solcher bewußt bin" zuschreiben kann, setzt voraus, daß sie ohne Ausnahme unter der Bedingung transzendentaler Einheit der Apperzeption stehen. Anders gewendet: Es gibt keinen Fall von Vorstellung, die ich mir als meine Vorstellung zuschreibe, wenn sie nicht in einer Mannigfaltigkeit von Vorstellungen auftritt, die ich mir als meine Vorstellungen zuschreiben könnte. ${ }^{19}$ Wenn es nun zutrifft, daß Vorstellungen, die ich als meine betrachte, immer schon unter der transzendentalen Einheit der Apperzeption und damit unter der

19 Vgl. dagegen die These Cramers (1987) 174f., "daß die Eingangsüberlegung des §16 ["Das: Ich denke, muß alle meine Vorstellungen begleiten können" (B131).] von dem, was Kant wenige Zeilen später die 'transzendentale Einheit des Selbstbewußtseins' nennt, 'um die Möglichkeit der Erkenntnis a priori aus ihr zu bezeichnen' [...], noch gar nicht spricht - und eben deshalb auch noch nicht von 'Synthesis'. [...] Das bloße Bewußtsein der Meinigkeit von Vorstellungen läßt sich nicht im Rekurs auf das Bewußtsein der Identität meiner selbst mit Bezug auf eine Pluralität von Vorstellungen, die ich insgesamt meine nenne, aufklären, und zwar genau deshalb nicht, weil ich sie insgesamt meine nenne. Dieser Einsicht steht nicht entgegen, daß jenes Bewußtsein, soviel wir von uns wissen, niemals ohne dieses vorkommt." Cramers Interpretation geht offensichtlich von der Annahme aus, daß sich das Bewußtsein des 'Ich denke' (B131f.) auch unabhängig von seiner Funktion als transzendentale Einheit so rekonstruieren lassen muß, daß die Selbstzuschreibung von Vorstellungen auch ohne Bezug auf die synthetische Einheit, die das 'Ich denke' für alle meine Vorstellungen hat, einsichtig wird. Cramer sieht sich zu dem Schluß berechtigt, die Einfachheit, die das 'Ich denke' als Einheitsprinzip auszeichnet, auf den Begriff des 'ich' als einen singulären Terminus zu übertragen: "er ist der Begriff von etwas Einzelnem, das in keiner Anschauung präsentierbar ist." (200) So bestechend die Schlußfolgerung auch ist, "daß der in einem solchen Urteil ['Ich weiß, daß ich x' als Reformulierung des 'Ich denke'] auftretende singuläre Terminus 'ich' der Begriff von etwas ist, das sich nur denken und auf keine andere Weise zu Bewußtsein bringen läßt" (202), so ist einzuwenden, daß das 'ich' als singulärer Terminus nach $L \S 1$ (AA IX $91=$ WW III 521) gerade keinen Begriff, sondern eine Anschauung bezeichnet. Wie aus einer Anm. der $M A N W$ (AA IV 542f. = WW V 108) hervorgeht, ist Kant zufolge "Das Ich, das allgemeine Korrelat der Apperzeption und selbst bloß ein Gedanke, [...] gar kein Begriff, sondern nur innere Wahrnehmung". M.E. kann der Ausdruck des 'Ich denke' nicht unabhängig von der dreifachen Kennzeichnung der Einheit als rein, ursprünglich und transzendental - das sind verschiedene Aspekte ein und desselben Prinzips der Begründung objektiver Erkenntnis - expliziert werden. 
Bedingung objektiver Erkenntnis stehen, dann scheint auch Schellings These gerechtfertigt zu sein, daß Kant das Bewußtsein des 'Ich denke' nicht anders als dasjenige Selbstbewußtsein versteht, das "Objecte denkt". Diese transzendentale Einheit des Selbstbewußtseins nennt Kant "darum objektiv", weil sie "alles in einer Anschauung gegebene Mannigfaltige in einen Begriff vom Objekt vereinigt" (B139). Daher besagt der "Grundsatz der synthetischen Einheit der Apperzeption" (B136),

\footnotetext{
"daß alle meine Vorstellungen in irgendeiner gegebenen Anschauung unter der Bedingung stehen müssen, unter der ich sie allein als meine Vorstellungen zu dem identischen Selbst rechnen, und also, als in einer Apperzeption synthetisch verbunden, durch den allgemeinen Ausdruck Ich denke zusammenfassen kann." (B138)
}

Die doppelte Hinsicht auf die Identität des erkennenden Subjekts und den Einheitsgrund jeder objektbezogenen Anschauung weist die synthetische Einheit des Bewußtseins zugleich als "objektive Bedingung aller Erkenntnis" (B138) aus. Diese Engführung wohlgemerkt des "obersten Prinzips alles Verstandesgebrauchs" (B136) begründet Kant damit, daß durch die Vorstellung "Ich bin" - so geht Kant in B138 unvermutet von der Rede über das 'Ich denke' zum 'Ich bin' über - "noch gar nichts Mannigfaltiges gegeben ist." (B138)

Für Kant ist das oberste Prinzip allen Verstandesgebrauchs zugleich "der Verstand selbst" (B134*); daher ist "der menschliche Verstand, der bloß denkt, nicht anschaut" (B139) zugleich "Bedingung alles Denkens" (B138). Da nun einerseits die Anschauung "diejenige Vorstellung" heißt, "die vor allem Denken gegeben sein kann" (B132), auf die andererseits zur Gewinnung und Formulierung von Erkenntnis "alles Denken als Mittel abzweckt" (A19 B33), gelingt Kant die methodische Trennung dieser beiden erkenntniskonstitutiven Momente. Doch es ist gar nicht dieser vielbeschworene Hiat zwischen den Funktionen des Denkens und der Anschauung als den Funktionen der Vorstellungskraft und Vorstellungsfähigkeit, der Schelling das Kantische Prinzip der Transzendentalphilosophie als mangelhaft bestimmt erkennen läßt. Für Schelling ist das Prinzip nicht "Bedingung alles Denkens" (B138), sondern es "beschreibt die Sphäre alles Denkbaren"20. Wie aus dem Titel der Abhandlung Vom Ich hervorgeht, bezeichnet das Prinzip "das Unbedingte im menschlichen Wissen", das als solches wie bei Kant zunächst an der Funktion des Denkens, nicht der Anschauung orientiert ist.

Als "Bedingung alles Denkens" bezieht Kant das Prinzip auf die aktive Funktion des Denkens. Es macht eine Aussage über den Denkenden als Akteur, der im Akt des Denkens vorausgesetzt ist. Als "Sphäre alles Denkbaren" bezieht Schelling das Prinzip auf den Gegenstand des Denkens. Damit verschiebt sich der Akzent des Geltungsbereichs des Prinzips, der sich auf das Prinzip selbst erstrecken soll. Die Differenz zu Kant besteht nun genau darin, daß das Ich nicht nur dann von sich weiß, wenn es immer schon auf Objekte bezogen gedacht wird. In Schellings Konzeption ist das Ich selbst Objekt des Denkens. Das Sein des Ich wird durch sein Gedachtwerden ausgedrückt: "Was Object ist, erwartet seine Existenz von etwas, das ausser der Sphäre seines blossen Gedachtwerdens liegt; das 
Ich allein ist nichts, ist selbst nicht denkbar, ohne daß zugleich sein Seyn gesezt werde, denn es ist gar nicht denkbar, als insofern es sich selbst denkt, d.h. insofern es ist." ${ }^{11}$

Das Konzept der transzendentalen Theorie der Erfahrung besagt, daß die Bedingungen der Möglichkeit objektiver Erkenntnis zugleich den Bedingungen der Möglichkeit der Gegenstände solcher Erkenntnis entsprechen. Diese Entsprechung gewährleistet das Prinzip der Apperzeption, das sich in der Identität des 'Ich denke' in der Mannigfaltigkeit der objektiven Vorstellungen ausdrückt. (A158 B197) Es ist dieser Grundgedanke Kants, den Schelling im Umfeld der fraglichen Begriffsbestimmungen in $\S 6$ der Abhandlung Vom Ich gerade als 'Gleichsetzung' deutet. Er drückt dies so aus: "Das theoretische Ich nemlich strebt, Ich und Nicht-Ich gleich zu sezen, also das Nicht-Ich selbst zur Form des Ichs zu erheben".22 Zwar spricht Schelling an dieser Stelle (noch) nicht von Konstruktion; doch weist die Debatte um die Begründung von Objektivität gerade auf den Problembestand, der es Schelling später erlauben wird, jenes 'Gleichsetzen' von Entgegengesetztem durch den Ausdruck Konstruieren zu ersetzen.

\section{3. 'konstruieren' als 'ursprünglich vorstellen': Becks Interpretation des Kantischen Konstruktionstheorems (1796)}

Schelling kritisiert Kants Begriff des Empirischen nicht mehr in der Binnenperspektive der Transzendentalphilosophie, sondern in der ihrer Voraussetzungen. Genaugenommen handelt es sich um eine Radikalisierung des transzendentalen Ansatzes, der nicht verlassen, sondern vertieft werden soll. Diese Absicht läßt Schelling bei der Einführung des Methodenbegriffs geradezu von der "Foderung einer ursprünglichen (transscendentalen) Construction"23 sprechen. Den Begriff der Konstruktion gebraucht Schelling erstmals in der Allgemeinen Übersicht der neuesten philosophischen Literatur $(1797 / 98)^{24}$, deren Beiträge er im Philosophischen Journal einer Gesellschaft Teutscher Gelehrten anonym veröffentlicht. Das Journal, das von Niethammer und Fichte herausgegeben wird, ist ein wichtiges Forum der frühidealistischen Debatte um die Grundlagen der Kantischen Transzendentalphilosophie. ${ }^{25}$ In dieser Debatte gewinnt nun Schellings Auseinandersetzung mit Becks Theorie des "ursprünglichen Vorstellens" zentrale Bedeutung für die Transformation des Konstruktionsbegriffs. Beck trägt diese Theorie im dritten Band (1796) seines Erläuternden Auszuges aus den critischen Schriften des Herrn Prof. Kant vor, der unter dem eigenen Titel erscheint: Einzig-möglicher Standpunct, aus welchem die critische Philosophie beurtheilt werden muß. ${ }^{26}$

$21 \quad$ Vom Ich §3: SW I 168 = AW II 91.

22 Vom Ich: SW I 176f. = AW II 101.

23 Übersicht: SS I 451 = AW IV 177.

24 Titel in späteren, um einige Beiträge der Übersicht gekürzten Ausgaben: Abhandlungen zur Erläuterung des Idealismus der Wissenschaftslehre; vgl. den "Editorischen Bericht" in AW IV 9-18.

25 Henrich (1991) 9-46 hat ein "Konstellationsforschung" genanntes Programm zur Analyse dieser Debatte entwickelt.

26 Fragmente dieser Abhandlung veröffentlicht Beck erstmals im Philosophischen Anzeiger 24-31 (1795) 185-246 der Annalen der Philosophie. Bde. I und II des Erläuternden Auszugs werden ebenfalls in den 
Mit der Theorie des ursprünglichen Vorstellens griff Beck in die Debatte um die fehlenden Grundlagen der Kantischen Vernunftkritik ein. Sein Konzept eines Postulats des ursprünglichen Vorstellens sollte zwar Reinholds Ansatz, den Begriff der Vorstellung als Fundamentalbegriff der Transzendentalphilosophie auszuweisen, nochmals vertiefen, zugleich aber Reinholds gescheiterten Versuch umgehen, die fehlende Prämisse als theoretischen Grundsatz, formuliert als "Satz des Bewußtseins", zu interpretieren. ${ }^{27}$ Darüber hinaus sollte dieses Konzept Jacobis Einwänden gegen den "Mechanismus des Begreifens", den er in der einseitigen Beanspruchung monokausaler Denkstrukturen sah, begegnen. ${ }^{28}$ Schon in der Einführung der Fragmente aus einer noch ungedruckten Schrift in den Annalen der Philosophie weist der Herausgeber darauf hin, daß Beck in der Diskussion einen metatheoretischen Standpunkt bezieht: Becks Abhandlung "hat nicht zur Absicht ein neues Princip der Philosophie aufzustellen oder einen obersten Grundsatz zu suchen; sondern sie will dieses Streben nach einem solchen Einzigen Princip selbst prüfen". ${ }^{29}$

Becks "Einzigmögliche StandPunktsLehre" - so nennt sie Schelling mit Reinhold - scheint Schelling über die Kritik Reinholds aus dessen Auswahl vermischter Schriften (1797) kennengelernt zu haben. ${ }^{30}$ Schelling zufolge habe schon Reinhold darauf hingewiesen, daß Beck in Kants Begriff des Empirischen die "schwache Seite der kritischen Philosophie" ausgemacht habe. Reinhold schreibt:

\footnotetext{
"Die Transcendentalphilosophie steht noch lange nicht als Wissenschaft fest, wenn sie von dem Empirischen keine andere Rechenschaft geben kann, als daß sie dasselbe zu ihrer eigenen Realität bloß voraussetzt, und gleichsam postulirt. Als Wissenschaft muß sie einen schlechthin transcendentalen Grund und Boden haben; und es fehlt ihr gänzlich an demselben, so lange sie bey der Deduktion ihres Inhalts von der äußeren Empfindung als einer Thatsache ausgehen muß, ohne dieselbe selbst deduciren zu können." ${ }^{31}$
}

In der Übersicht führt Schelling diese Auseinandersetzung über die nun von Reinhold vermittelte Kritik an Becks Theorie des ursprünglichen Vorstellens. Diese Auseinandersetzung ist insofern für die hier verfolgte Fragestellung von Bedeutung, als Schelling im Zuge seiner Kritik an Beck den Konstruktionsbegriff einführt und, wie sich zeigen läßt, unabhängig von Reinhold den Begriff aus Becks Interpretation der MANW übernimmt. ${ }^{32}$ Beck selbst macht seine Kritik des Begriffs des Empirischen an $K r V \S 1$ fest.

Annalen der Philosophie 73-74 (1795) 577-589 rezensiert; Bd. III wird bereits in 1 (1796) 17-81 ausführlich 'beurteilt'.

27 Den Satz des Bewußtseins führt Reinhold im Versuch einer neuen Theorie des menschlichen Vorstellungsvermögens (1789) 200 in einer vorläufigen Fassung ein. In der verbesserten Formulierung des Fundaments des philosophischen Wissens (1791) 78 lautet der Satz: "[...] durch bloße Reflexion über die Thatsache des Bewußtseyns, das heißt, durch Vergleichung desjenigen, was im Bewußtseyn vorgeht, wissen wir: daß die Vorstellung im Bewußtseyn durch das Subjekt vom Objekt und Subjekt unterschieden und auf beyde bezogen werde."

$28 \mathrm{Zu}$ Jacobis in der "Beylage VII" der Spinoza-Briefe (1789) vertretenen These, daß die philosophische, über Begriffe vermittelte Demonstration kein Erkennen wirklicher Sachverhalte erlaubt, sondern stets aporetisch verläuft, s. 9.2.

29 Annalen der Philosophie 24 (1795) 185.

30 Übersicht: SW I 414 = AW IV 141; vgl. die Anm. der Herausgeber in AW IV 352.

31 Reinhold (1797) 320.

32 Ich vermute daher, daß Schelling schon 1795 aus erster Hand über Becks Fragmente unterrichtet war, zumal eine Rezension seiner eigenen Abhandlung Über die Möglichkeit der Form einer Philosophie 
Ich gehe daher von $\$ 1$ der "Transzendentalen Elementarlehre" der $K r V$ aus, in dem Kant die Begriffe 'rein', 'empirisch', 'a priori' und 'a posteriori' mit Bezug auf sein transzendentales Begründungsprogramm der Erfahrung einführt. Darauf weist Kant selbst hin:

"Ich nenne alle Vorstellungen rein (im transzendentalen Verstande), in denen nichts, was zur Empfindung gehört, angetroffen wird." (A20 B34)

"Diejenige Anschauung, welche sich auf den Gegenstand durch Empfindung bezieht, heißt empirisch. [...] Da das, worinnen sich die Empfindungen allein ordnen, und in gewisse Form gestellt werden können, nicht selbst wiederum Empfindung sein kann, so ist uns zwar die Materie aller Erscheinung nur a posteriori gegeben, die Form derselben aber muß zu ihnen insgesamt im Gemüte a priori bereitliegen, und daher abgesondert von aller Empfindung können betrachtet werden." (A20 B34)

Es fällt auf, daß die Bestimmungen bei Kant durchweg an der Beziehung zum Empfindungsbegriff orientiert sind. Dabei bezeichnet 'rein' etwas, das sich auf "nichts, was zur Empfindung gehört" bezieht, 'a priori' etwas, das als "abgesondert von aller Empfindung" betrachtet wird. Im Gegensatz zu diesen negativen Kennzeichnungen deutet der Begriff 'empirisch' auf etwas, das positiv "durch Empfindung", genauer als Bedingung der Beziehung auf einen Gegenstand bestimmt ist. Entsprechend gilt Empfindung unter dem reflexionslogischen Titel der Materie als etwas, das "nur a posteriori gegeben" ist. Der Weise, in der Kant den Empfindungsbegriff auszeichnet, liegt das Verfahren der Dekomposition der Einen Erfahrung in ein Ensemble der Konstitutionsbedingungen solcher Erfahrung zugrunde. Innerhalb dieser Bedingungen kommt dem Empfindungsbegriff jedoch eine ambivalente Funktion zu: Sofern Empfindung "die Wirkung eines Gegenstandes auf die Vorstellungsfähigkeit" (A19f. B34), will sagen, Sinnlichkeit und Rezeptivität bezeichnet, ermöglicht sie die materiale Bestimmung von Erfahrung in der Weise ihrer Gegebenheit. ${ }^{33}$ Als Moment der Sinnlichkeit gehört Empfindung zu den notwendigen Bedingungen, ohne die die Anwendung der Kategorien auf Anschauungsgehalte nicht vollzogen und den kategorial vermittelten Erkenntnissen objektive Realität nicht zugesprochen werden kann. Daher hebt Kant hervor, daß, "wenn man diese Bedingung wegnimmt, alle Bedeutung, d.i. Beziehung aufs Objekt, wegfällt." (A241 B300)

Es ist festzuhalten, daß bei Kant über die positive Kennzeichnung des Empirischen "durch Empfindung" auch die Begriffe 'rein', 'a priori' und 'a posteriori' nach ihrer "Beziehung aufs Objekt" bestimmt werden. Kant weist dem Moment der Empfindung nun eine Funktion zu, die sie zum nicht weiter durch Begriffe beschreibbaren und antizipierbaren Ausgangspunkt der transzendentalen Theorie der Erfahrung und in der Folge auch zum Ansatzpunkt der frühidealistischen Kant-Kritik macht. Empfindung bezeichne "das Eigentlichempirische der sinnlichen und äußeren Anschauung, weil es gar nicht a priori gegeben werden kann". ${ }^{34}$

überhaupt (1794) ebenfalls in den Annalen der Philosophie 4 (1795) 29-32 erschienen war; vgl. den "Editorischen Bericht" in AW IV 259 und Schellings Erklärung zu der Rezension in AW IV 301-311. Anders als Reinhold geht der Rezensent des dritten Bandes des Erläuternden Auszugs in den Annalen der Philosophie 1 (1796) 50-59 ausführlich auf Kants MANW ein.

33 "Vermittelst der Sinnlichkeit werden uns Gegenstände gegeben." (A19 B33)

34 MANW: AA IV $481=$ WW V 26. 
Als notwendig erschlossene Bedingung der "Beziehung aufs Objekt" kann das Eigentlichempirische in der Kantischen Theorie jedoch nur vorausgesetzt, nicht deduziert werden. Gleichwohl bildet es einen konstitutiven Bestandteil objektiver Erkenntnis.

Beck selbst macht seine Kritik an einer, wie er meint, zwar naheliegenden, aber ganz unkantischen Lesart des Begriffs der Empfindung in $\S 1$ der $K r V$ fest. Danach könne die Differenz von Begriff und Anschauung nicht so verstanden werden, daß "die unmittelbare Vorstellung selbst des Objects Anschauung, mithin mit Vorstellung des Einzelnen einerley [ist]; ein Begriff dagegen ist lediglich die Vorstellung, die alle Objecte unter sich begreift." ${ }^{35}$ Nach einer solchen Lesart könne weder Kants These der Verknüpfung von Anschauung und Begriff $\mathrm{zu}$ Erkenntnis eingesehen noch das Auftreten empirischer Anschauungen durch einen die Sinne affizierenden Gegenstand anders als durch die dogmatische Voraussetzung der berüchtigten 'Dinge an sich' gedeutet werden. Die entscheidende Frage: "was verbindet meine Vorstellung von diesem afficirenden Gegenstande mit demselben?" bleibe dann jedoch ungelöst. ${ }^{36}$ Vor der Darstellung der Kritik an dieser unkantischen Lesart sind zunächst die Schwierigkeiten zu benennen, die sich für Beck aus Kants Bestimmungen der Begriffe 'rein', 'empirisch', 'a priori' und 'a posteriori' ergeben.

\footnotetext{
"Bleibt man dabey stehen, daß man die Kategorien für Begriffe ansieht, dann geht wahrlich aller Unterschied zwischen dem was a priori, und demjenigen, was a posteriori heißen soll, ganz verloren. Denn man sieht sie so an, wenn man durch Beylegung gewisser Bestimmungen, die in ihnen gedacht werden, Objecte sich vorstellt, und in dieser Hinsicht ist es doch wohl gleich viel, ob man einen Gegenstand durch die Bestimmungen: roth, hart, flüssig, oder ob man ihn als Größe, Reales, Substanz u.s.w. vorstellt. Als ein Zeichen dieses Unterschiedes die Empfindung angeben und denjenigen Begriff a priori nennen, der von Empfindung frey ist, den aber für einen empirischen halten, in welchem sich Empfindung befindet, bedeutet gar nichts. Denn die Empfindung selbst ist doch niemahls der Begriff; und da könnte wohl am Ende mit demselben Rechte gesagt werden, daß auch die für reine Begriffe ausgegebenen Kategorien eben so wohl Empfindung enthalten als die empirischen "17
}

Beck weist darauf hin, daß Kant die fraglichen Bestimmungen an ihrem Bezug zum Empfindungsbegriff orientiert. Nun sei aber der Unterschied zwischen den reinen Verstandesbegriffen und empirischen Begriffen von Qualitäten bedeutungslos, wenn man bei der Auffassung stehen bleibe, "daß man Kategorien für Begriffe ansieht"; und das sei dann der Fall, "wenn man durch Beylegung gewisser Bestimmungen, die in ihnen gedacht werden, Objecte sich vorstellt".

Beck behauptet nicht, daß die qualitativen Eigenschaften von Gegenständen gleichzusetzen seien mit den Bestimmungen, die, durch die Kategorien bezeichnet, uns überhaupt erst Gegenständlichkeit vorstellen lassen, auf die bezogen Qualitäten als Bestimmungen an Objekten ausgesagt werden können. Beck behauptet jedoch, daß der Unterschied zwischen den Begiffen 'a priori' und 'a posteriori' bedeutungslos sei, wenn man diese durch Kategorien wie durch Empfindungen bezeichnete Eigenschaften als auf Begriffe gebrachte

35 Beck (1796) 366.

36 Beck (1796) 367. Zur Formulierung dieser Fragestellung in Kants Brief an Markus Herz vom 21.2.1772 vgl. 6.1. Zur Kantischen Differenz von Begriff und Anschauung aus der Sicht Becks s. Heller (1993), der auf die frühidealistische Debatte jedoch nur am Rande eingeht.

37 Beck (1796) $176 f$. 
Gegenstandsbestimmungen betrachtet. Aus der Differenz der Begriffe 'a priori', der etwas, das "von Empfindung frey ist", bezeichnet, und 'a posteriori' oder 'empirisch', der etwas, "in welchem sich Empfindung befindet", bezeichnet, folgt noch nicht die Differenz des Verstandesbegriffs der Qualität und des durch den Begriff Bezeichneten.

"Denke ich aber ein Object durch den Begriff: Größe, so bin ich eben so weit als wenn ich es als ein rothes Ding denke. In diesem Denken liegt schon das Ding, (Object,) gleichsam im Hintergrunde und alles Denken ist darin ganz einerley, daß es schon eine objective Einheit voraus setzt. Diese Einheit im bloßen Begriffe ist [...] die analytische." 38

Begriffe von Objekten zu haben, bedeutet für Beck, Merkmale, durch die ich ein Objekt bestimme, unter den Begriff von diesem Objekt zu subsumieren. In dieser Leistung der Subsumtion besteht für Beck das Denken, das als solches immer schon eine "objective Einheit" des durch es Gedachten voraussetzt. Diese Einheit ist in Begriffen ausgedrückt, durch deren Verbindung im Urteil Objekte "durch Beylegung gewisser Bestimmungen" vorgestellt werden.

"Wenn ich mir etwas vorstelle, so geschieht es durch Beylegung gewisser Bestimmungen, die ich aus dem ursprünglichen Vorstellen heraus hebe, das heißt: alles etwas vorstellen ist ein Denken." 39

Das Denken, das sich als "etwas vorstellen" im Urteil "durch Beylegung gewisser Bestimmungen" vollzieht, bezeichnet Beck in Abgrenzung zum Begriff des ursprünglichen Vorstellens als ursprüngliche Anerkennung: "Object, objective Einheit, das ist es, was ich in der ursprünglichen Anerkennung erhalte." ${ }^{40}$ Vorstellen als "etwas Vorstellen" beruht auf dem logischen Verstandesgebrauch, der in der von Kant so genannten analytischen Einheit des Bewußtseins ausgedrückt wird. Dieser Einheit gehe die "ursprünglich-synthetisch objektive Einheit des Bewußtseins" - so faßt Beck die Funktionen der transzendentalen Apperzeption zusammen - als höchster Punkt allen Verstandesgebrauchs voraus. Vorstellen als "ursprüngliches Vorstellen" entspreche nun der ursprünglichen Synthesis, die in der ursprünglichen Anerkennung zum "etwas Vorstellen", d.h. zur Bestimmung eines Begriffs von einem Objekt, "fixirt" werde.

Die synthetische Einheit werde durch die Kategorien vermittelt, die Beck daher auch nicht als Begriffe von Objekten angesehen wissen will: "Das ursprüngliche Vorstellen besteht in den Kategorien. Dieselben sind nichts anderes als ursprüngliche Vorstellungsarten." ${ }^{41}$ In dieser Absicht unterscheidet er zwischen Vorstellungsobjekt und Vorstellungsakt, wobei das vorgestellte Objekt bloß eine Modifikation des Vorstellungsaktes, den er ursprüngliches Vorstellen nennt, sein soll.

\footnotetext{
"Unser Verstand ist so beschaffen, daß er sich die Objecte jederzeit durch Begriffe, also in einer analytischen Einheit, und nicht in der ursprünglich-synthetischen Einheit des Bewußtseyns vorstellt. Wenn wir uns nämlich Objecte vorstellen, so haben wir immer einen Begriff von den Dingen; das ist: durch Beylegung gewisser Bestimmungen fixiren wir einen Beziehungspunct und heften so gleichsam das Object an einige Merkmahle. In dem ursprünglichen Vorstellen stellen wir uns eigentlich kein Object vor. Der Verstand erzeugt dadurch die ursprünglich-synthetische objective
} 
Einheit, die freylich alle Bedeutung eines Begriffs constituirt, die aber doch erst in eine analytische Einheit, das ist: in den Begriff übergehen muß, damit der Gegenstand vorgestellt werde." ${ }^{42}$

Nun behauptet Beck, daß "die Anwendung der Kategorien auf Objekte der Anschauung, d.i.: die Vorstellung dieser Gegenstände durch die Kategorien als Bestimmung derselben, auf dem ursprünglichen Verstandesgebrauch der Kategorien beruht, in welchem das, was Anschauung heißt, und was Kategorie, (als ursprüngliches Vorstellen,) ist, zusammen fällt, und nicht so weit, wie mancher glauben möchte, von einander entfernt ist." ${ }^{43}$ Schon der Begriff des 'Zusammenfalls' von Anschauung und Kategorie, verstanden als Vorstellungsart, legt die Vermutung nahe, daß Beck darauf hinaus will, die Differenz zwischen beiden durch deren Gleichsetzung aufzuheben. Becks Theorie eröffnet denn auch eine Perspektive, in der "das Anschauen die ursprüngliche Synthesis des Gleichartigen ist, mithin mit der Kategorie der Größe, (als ursprünglichem Verstandesgebrauche,) einerley ist." ${ }^{44} \mathrm{Im}$ Rückgriff auf diese These der Identität des Anschauens und der Kategorie der Größe kann Beck dann auch behaupten, die geometrische Konstruktion sei "ein ursprüngliches Vorstellen und nicht ein Verfahren mit Begriffen [...], das ist: das reine Anschauen, die Construction". 45

Becks Interpretation des Kantischen Konstruktionstheorems auf der Grundlage der Theorie des ursprünglichen Vorstellens erlaubt nicht nur die Explikation des Begriffs des ursprünglichen Vorstellens durch den der Konstruktion. Konstruktion, wohlgemerkt als mathematisches Verfahren in einer philosophischen Interpretation, beschreibt so die Grundlage dessen, was uns im Denken im Gebrauch von Begriffen vertraut ist. Mit diesem mathematischen Modell zur Beschreibung unseres durch Begriffe vermittelten Verstehens von Sachverhalten sieht Beck zugleich die Art und Weise vorgegeben, wie der methodische Zugriff auf die Konstitutionsbedingungen des Denkens eröffnet werden kann. Als Postulat im Sinne des Geometers liegt das ursprüngliche Vorstellen nämlich dem begrifflichen Verstehen insofern voraus, als es "alle[n] Sinn und alle Bedeutung unsrer Begriffe [...] constituire. ${ }^{46}$

Sofern nun, so schlägt Beck vor, "das oberste Princip alles Verstandesgebrauchs das Postulat sey: ursprünglich vorzustellen"47, müsse auch der Anfang der Philosophie mit einem Postulat gemacht werden: "wie der Geometer, der seinen Gegenstand, den Raum, erzeugt". 48 "Man kann daher die Transcendental-Philosophie ganz passend eine Kunst, sich selbst zu verstehen, nennen, und unser Postulat als das Princip alles Verständlichen ansehen." 49 Vor dieser knappen Skizze der Theorie Becks ist nun die zwar polemische, doch differenzierte Kritik Schellings vorzustellen. Daran soll gezeigt werden, daß und auf

42 Ebd. 148f.

43 Ebd. 413.

44 Ebd. 367.

45 Ebd. 359f.; vgl. 197: "Die Mathematik ist die Wissenschaft durch Construction der Begriffe. Einen Begriff aber construiren heißt, die ihm correspondirende Anschauung a priori darstellen. Wenn wir unter der reinen Anschauung die ursprüngliche Größenerzeugung, die ursprüngliche Synthesis des Gleichartigen, verstehen [...]".

46 Beck (1796) 147.

47 Ebd. 152.

48 Ebd. 137; vgl. 123f., 165.

49 Ebd. 139. 
welche Weise es Schelling gelingt, im Konzept mathematischer Konstruktion ein Modell philosophischer Theorie zu erkennen.

\section{4. 'ursprünglich vorstellen' als 'ursprünglich (transzendental) konstruieren': Schellings Interpretation der Theorie des ursprünglichen Vorstellens (1797)}

Im Zuge seiner Kritik an Becks Theorie des ursprünglichen Vorstellens überführt Schelling das bei Beck immer nur philosophisch interpretierte Verfahren mathematischer Konstruktion in einen Methodenbegriff der Philosophie. Zwar habe Beck, so hatte schon Schelling mit Reinhold betont, am problematischen Status des Empfindungsbegriffs die "schwache Seite der kritischen Philosophie" aufgedeckt; doch habe Becks Interpretationsansatz den Unterschied von Sinnlichkeit und Verstand nicht erklärt, sondern zum Verschwinden gebracht. Ebensowenig habe Beck "das Reale d.h. die Empfindung in unsern Vorstellungen" erklären können, da er im Begriff des Vorstellens "keine andre als ideale Handlungen vorstellig zu machen weiß, und mit klaren Worten behaupten muß, daß die Empfindung eine Handlung des ursprünglichen Verstandes sey". ${ }^{50}$

Schelling bezieht sich mit diesem Einwand auf die von Beck für unkantisch gehaltene Ansicht, wonach die reinen bzw. formalen Anschauungen, Raum und Zeit, und die empirischen bzw. materialen Anschauungen, das Reale in der Empfindung, "vor der ursprünglichen Synthesis und Anerkennung gegebenes Mannigfaltige"51 bezeichne. Es bestehe vielmehr in der ursprünglich-synthetischen Einheit des Bewußtseins: "Ich synthesire darin meine Empfindung. In dieser Synthesis [...] erzeuge ich die Zeit. Das Bestimmen dieser Zeit, wodurch diese Synthesis selbst fixirt wird, ist die ursprüngliche Anerkennung. In diesem ursprünglichen Vorstellen wird die ursprünglich-synthetische Einheit des Bewußtseyns, nämlich der Begriff, wodurch ich das Reale eines Dinges denke, erzeugt." ${ }^{52}$ Nun - so führt Schelling seinen Einwand fort - sei jedoch der Verstand, von Beck nicht anders als von Kant als "Vermögen der Begriffe" definiert, selbst nur ein abgeleitetes Vermögen und daher gar nicht "als ursprünglich zu denken." ${ }^{53}$

\footnotetext{
"Wir wissen gar wohl, daß alle auch die ursprünglichen Handlungen des Geistes, wenn wir vom Standpunkt des Bewusstseyns aus darüber reflectiren, als lediglich ideal erscheinen. Aber wir müssen auch davon wieder den Grund angeben, um so mehr, da das Ideale gar nicht anders als im Gegensatz gegen das Reale gedacht werden kann. Ideales und Reales aber, (Vorstellung und Objekt, Begriff und Anschauung,) scheiden sich selbst nur im Bewusstseyn; jenseits des Bewusstseyns also muß zwischen idealem und realem Handeln gar kein Unterschied seyn." ${ }^{54}$
}

Schelling führt gegen Becks Theorie an, daß sie, ausgehend vom Begriff des Vorstellens, immer nur wieder vom Standpunkt des Bewußtseins reflektiert. Daher heiße "ursprünglich vorstellen" soviel als "daß man jenseits des Bewusstseyns mit Bewusstseyn vorstelle, was

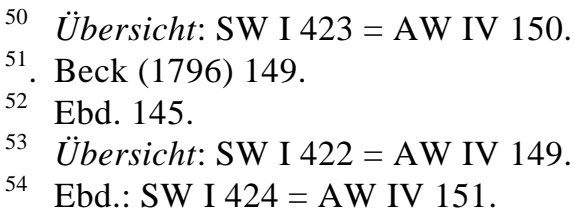


ungereimt ist." ${ }^{55}$ Es fällt auf, daß Schelling in der Auseinandersetzung mit Beck bei der Wiedergabe einzelner Theoreme der Standpunktslehre immer wieder auf den Begriff der Konstruktion zurückgreift, um den Begriff schließlich mit der Formel der "Foderung einer ursprünglichen (transscendentalen) Construction"56 als ein philosophisches Verfahren zu etablieren. Die direkte Gleichsetzung der Begriffe 'ursprünglich vorstellen' und 'ursprünglich konstruieren' formuliert Schelling im Kontext seiner Überlegungen der Übersicht, die dem "Zusammenhang der theoretischen und praktischen Philosophie"57 gelten:

"Daß jenes ursprüngliche Vorstellen - jenes ursprüngliche Construiren - nicht bloß ideal, sondern real und ursprünglich-nothwendig sey, kann ich niemand begreiflich machen, ohne ihm das innere Princip alles Vorstellens und Construirens aufzuschließen." ${ }^{58}$

Es ist die besondere hermeneutische Situation in der Vermittlung dieses inneren Prinzips, die Schelling in Form und Artikulation des Prinzips an Becks Methodologie anknüpfen läßt: "Jenes ursprüngliche SelbstBestimmen des Geistes nun kann ich allerdings in einem Grundsatze ausdrücken. Dieser Grundsatz aber ist in Bezug auf den, mit dem ich rede, nothwendig ein Postulat" ${ }^{59}$.

"Eine Wissenschaft aber, die ihrer Natur nach transscendental ist, muß in ihrem ersten Princip schon allen Empirismus ausschließen. Dieß thut z.B. die Geometrie, indem sie die ursprünglichste Construction POSTULIRT, und dadurch den Lehrling gleich anfangs erinnert, daß er in der ganzen Wissenschaft nur mit seinen eignen Constructionen zu thun habe. Die Linie, das Object dieser ursprünglichen Construction existirt nirgends außer dieser Construction, sie ist nur diese Construction selbst. Eben so soll es in der Philosophie seyn; der Lehrling muß gleich anfangs in die transscendentale Denkart gleichsam versetzt werden. Also muß schon das erste Princip seine eigne Construction seyn, die man von ihm FODERT, (ihm selbst überlässt), damit er so von Anfang an lerne, daß, was ihm durch Construction entsteht, außer dieser Construction nichts sey, und überhaupt nur sey, inwiefern er construirt." ${ }^{60}$

Während Konstruktion bei Beck eindeutig als mathematischer Methodenbegriff, den es durch das 'ursprüngliche Vorstellen' sich verständlich zu machen gelte, gebraucht wird, wird Konstruktion bei Schelling, wie der Zusatz 'transzendental' anzeigt, zu einer philosophischen Methode verändert. Das folgende Textbeispiel ${ }^{61}$ soll am Problem der Begründung von Objektivität verdeutlichen, wie Schelling in der Auseinandersetzung mit Beck den Begriff des ursprünglichen Vorstellens durch den des Konstruierens ersetzt.

"Wir wissen gar wohl, daß der Verstand nichts anders ist, als das Vermögen der Begriffe, und daß Begriffe wiederum nichts anders sind, als Abstractionen von unsrer ursprünglichen AnschauungsWeise, daß also die Zergliederungen des reinen Verstandes, Kategorieen genannt, wirklich nichts anders ausdrücken, als die ursprüngliche und nothwendige HandlungsWeise des Geistes in der Anschauung, oder, weil das Object von dieser HandlungsWeise gar nicht verschieden ist, die ursprüngliche Synthesis, wodurch erst alles Object wird und entsteht." 
Schelling nimmt zunächst Kants Bestimmung auf, wonach Verstand das Vermögen der Begriffe sei, und führt den Begriff des Begriffs mit Beck auf den Begriff von Handlungsweisen des Geistes in der Anschauung zurück. Jenseits der Abstraktion von einer solchen Anschauungsweise sei das Objekt von der Handlungsweise selbst gar nicht verschieden: "Wenn aber das Object ursprünglich gar nichts anders ist, als eine bestimmte HandlungsWeise (Construction) unsers Geistes, so müssen wir doch diese HandlungsWeise uns entgegensetzen, weil ohne dies niemals die Vorstellung eines Objects in uns entstehen würde." Schelling spricht hier nicht von der Entstehung eines Objekts, sondern von der Entstehung der Vorstellung eines Objekts in uns. Konstruktion wird zwar als "eine bestimmte HandlungsWeise" und in dieser Bestimmtheit als Objekt aufgefaßt, doch nicht als eine Vorstellung von Objekten in uns, der sie vielmehr vorausgeht. Denn erst das Entgegensetzen dieser Handlung macht das Auftreten von Vorstellungen in uns als Vorstellungen von Objekten möglich.

"(Die StandPunktsLehre selbst behauptet: Es gebe kein ursprüngliches Vorstellen eines Gegenstandes, sondern bloß ein ursprüngliches Vorstellen; weil, wenn wir eine Vorstellung von einem Objecte haben, diese allemal schon Begriff sey). Dies können wir nun nicht, ohne von jener bestimmten HandlungsWeise zu abstrahiren. Dies ist Geschäft des Verstandes, und indem er dies thut, entsteht ihm der Begriff, d.h. die allgemeine Vorstellung von der HandlungsWeise des Geistes überhaupt."

In der Schrift Vom Ich hatte Schelling dargelegt, daß das theoretische Ich im Erkennen danach strebe, die durch ihre Entgegensetzung begründete Trennung von Nicht-Ich und darum empirisch-bedingtem Ich in der "Gleichsetzung" beider Momente aufzuheben. Die in der Erkenntnis ausgedrückte "Übereinstimmung" von Vorstellung und Gegenstand sollte so als Zurückführung auf eine (im absoluten Ich gegründete) ursprüngliche Identität erklärt werden. ${ }^{62}$ Schelling geht in der Übersicht davon aus, daß das Objekt, auch wenn es als bestimmte Handlungsweise betrachtet werde, nur unter der Voraussetzung der Entgegensetzung als "Vorstellung eines Objects in uns" auftritt. Geht der Vorstellung des Objekts die Entgegensetzung voraus, dann resultiert diese Vorstellung des Objekts in uns gerade aus der Gleichsetzung der Entgegengesetzten. ${ }^{63}$ So betont Schelling geradezu, daß "der menschliche Geist überhaupt genöthigt ist, alles, was er anschaut und erkennt, aus Entgegengesetzten zu construiren" ${ }^{164}$. Eben diese als Objekt bezeichnete bestimmte Handlungsweise nennt Schelling im Textbeispiel Konstruktion.

62 Schelling macht diese ursprüngliche Identität an dem Streben' des Ich fest, "seine Identität zu retten" (SW I 180* = AW II 104*).

63 Vgl. Übersicht: SW I 409f. = AW IV 136f.: "Der Verstand setzt weder das Object, noch das Object den Verstand, (als ruhendes Vermögen) voraus. Der Verstand (in construirender Thätigkeit) und das Object sind Eins und dasselbe, und unzertrennlich."

64 Übersicht: SW I 408 = AW IV 135. 


\section{5. 'ursprünglich vorstellen' als 'konstruieren': Becks zweiter Kommentar zu Kants MANW (1796)}

Die Überlegungen, vor deren Hintergrund Schelling den Begriff der transzendentalen Konstruktion einführt, gelten der Begründung der Philosophie aus einem Prinzip, von dem zugleich unser Verständnis von Objektivität herleitbar sein soll. Ansatzpunkt in der Schrift Vom Ich §6 ist Schellings Kritik an dem "gewöhnlich zu engen" Begriff des Empirischen. Kant zufolge wird das Empirische "durch Empfindung" bestimmt, deren Auftreten in uns als 'a posteriori' gegeben einfachhin vorauszusetzen ist. Sofern wir nur bei Affektion unserer Sinne Empfindungen haben, gehört Empfindung doch $\mathrm{zu}$ den notwendigen Bedingungen kategorial vermittelter Erkenntnisse, die sich auf Objekte als Gegenstände der Erfahrung beziehen. Nun setzt Schelling den Begriff der Konstruktion als Interpretament für Becks Theorem des ursprünglichen Vorstellens genau da ein, wo die Begründung von Objektivität verhandelt wird. Für Beck wird die Rede von Objekten der Erkenntnis als von Objekten der Vorstellungen in uns erst dann verständlich, wenn diese Vorstellungsinhalte als Modifikationen eines ursprünglichen Vorstellungsaktes aufgefaßt werden und als solche auf die "ursprünglich-synthetisch objektive Einheit" des Bewußtseins zurückführbar sind. Diese Einheit wird durch die Kategorien als Arten des ursprünglichen Vorstellens vermittelt und ist so konstitutiv für die Bedeutung dessen, über das wir in begrifflich vermittelter Form als objektive Erkenntnis verfügen. Was Beck als 'Zurückführung' auf eine ursprüngliche Synthesis deutet, wird bei Schelling der Sache nach zu einer 'Gleichsetzung' von empirisch-bedingtem Ich und Nicht-Ich, die er durch das Streben des Ich motiviert, "seine Identität zu retten". Im Zuge seiner Auseinandersetzung mit Beck in der Übersicht ersetzt Schelling das, was er in der Abhandlung Vom Ich noch als Gleichsetzung bezeichnete, durch Konstruktion.

So polemisch Schelling gegen den "Erläuterer der Kantischen Philosophie" auch vorgeht, so attraktiv muß ihm doch dessen Interpretation des Konstruktionsbegriffs erscheinen, daß er den Begriff, bei Beck stets mathematisch konnotiert, schließlich als philosophischen Methodenbegriff adoptiert. Eine Schlüsselrolle bei dieser Transformation des Konstruktionsbegriffs spielt, so meine These, Becks zweiter Kommentar zu Kants MANW, in dem Beck die Anwendung mathematischer Konstruktionen in der Naturwissenschaft durch Rückgriff auf die Theorie des ursprünglichen Vorstellens erläutert. ${ }^{65}$ Beck hat diesen Kommentar als integralen Bestandteil der Exposition seiner Theorie verstanden, mit dem als $§ 1$ er die "Beurtheilung der ganzen kritischen Philosophie aus dem transscendentalen Standpunkte der synthetisch-objectiven Einheit des Bewußtseyns" eröffnet. ${ }^{66}$ Da Reinhold in der Auswahl vermischter Schriften, nach deren zweiten Teil Schelling sonst den Beckschen Text zitiert ${ }^{67}$, diesen Kommentarteil übergeht, muß Schelling, veranlaßt durch seine naturphilosophischen Interessen und sein Studium der $M A N W^{68}$, unabhängig von

65 Der erste Kommentar in Bd. II (1794) des Erläuternden Auszugs ist von Becks Theorie des ursprünglichen Vorstellens noch unbeeinflußt.

66 Beck (1796) 206-344.

67 Vgl. die "Erklärenden Anmerkungen" der Herausgeber der Übersicht in AW IV 352.

68 Zu Schellings früher Rezeption der Naturwissenschaften seiner Zeit s. Heckmann u.a. (1985) 12-108, Jacobs (1988) 3-12. 
Reinhold auf den eigentümlichen Zusammenhang, den Beck zwischen dem ursprünglichen Vorstellen und der mathematischen Konstruktion sieht, gestoßen sein.

Kant wollte die $M A N W$ als "Probe" (B110) der in der $K r V$ entwickelten Prinzipien der Theorie objektiver Erkenntnis verstanden wissen.

\footnotetext{
"Und so tut eine abgesonderte Metaphysik der körperlichen Natur der allgemeinen vortreffliche und unentbehrliche Dienste, indem sie Beispiele (Fälle in concreto) herbeischafft, die Begriffe und Lehrsätze der letzteren (eigentlich der Transzendentalphilosophie) zu realisieren, d.i. einer bloßen Gedankenform Sinn und Bedeutung unterzulegen." ${ }^{69}$
}

Auch Beck leitet seinen Kommentar gerade mit Überlegungen zum paradigmatischen Status der $M A N W$ ein im Hinblick auf die Idee der Transzendentalphilosophie genannten Wissenschaft, deren Gegenstand, das ursprüngliche Vorstellen, zugleich Prinzip alles Verständlichen sei:

\begin{abstract}
"An die Betrachtung des Transcendentalen unsrer Erkenntniß schließt keine andere sich so vortrefflich an als die Betrachtung der metaphysischen Principien der Naturwissenschaft. Wir können keine Materie wählen, die so tauglich wäre, um den Leser mit dem Princip des ursprünglichen Vorstellens vertraut zu machen, als gerade diese, worin Kant den Begriff der Materie auf die ursprünglich-synthetische Einheit des Bewußtseyns zurück führt und so die Verständlichkeit und Haltung des Begriffs zeigt". ${ }^{70}$
\end{abstract}

Im Gegensatz zu seinem ersten Kommentar kommt es Beck nun darauf an, den systematischen Ort $\mathrm{zu}$ bezeichnen, von dem her sich verstehen läßt, wie der Konstruktionsbegriff durch den Begriff des ursprünglichen Vorstellens interpretiert werden kann, ohne daß Konstruktion jedoch anders als ein genuin mathematischer Methodenbegriff bestimmt wird. Möglicherweise ist Schelling diese Einschränkung entgangen. Von Bedeutung ist Becks Kommentar der MANW deshalb, weil er Schelling eine Perspektive eröffnete, den Konstruktionsbegriff so $\mathrm{zu}$ interpretieren, daß er als philosophisches Verfahren geeignet schien, die Begründungsprobleme der Kantischen Philosophie, die sich auch an der ambivalenten Funktion des Empfindungsbegriffs manifestierten, zu beseitigen. Becks Vorgehen läßt sich am Beispiel der MADyn verdeutlichen, das nach der Zielsetzung des Kommentars bis an den Punkt reicht, wo einerseits die Grenzen der philosophischen Herleitung der Dualität der Grundkräfte von Materie sichtbar werden und wovon andererseits der Mathematiker auf der Grundlage dieser Herleitung "sein Geschäft anfängt". ${ }^{71}$ Kants dynamische Erklärung des Begriffs der Materie lautet:

"Materie ist das Bewegliche, so fern es einen Raum erfüllt. Einen Raum erfüllen heißt allem Beweglichen widerstehen, das durch seine Bewegung in einen gewissen Raum einzudringen bestrebt ist. Ein Raum, der nicht erfüllt ist, ist ein leerer Raum. ${ }^{72}$

Beck geht davon aus, daß der Begriff der Materie als Begriff den Standpunkt der analytischen Einheit des durch ihn Bezeichneten repräsentiert. In dieser Hinsicht kennzeichnet er Kants kategoriale Zergliederung des Begriffs der Materie in den MANW als ein Verfahren, in dem der Begriff durch logische Analyse seiner Merkmale beschrieben

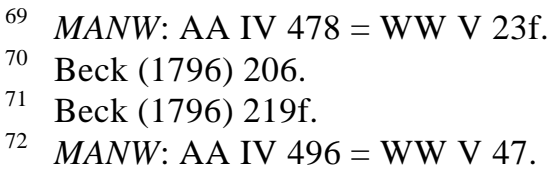


wird. Daß Kant bei dieser Beschreibung den Begriff der Materie nach dem Schema der Kategorientafel zergliedert, heißt für Beck, daß der Begriff in der kategorial vermittelten analytischen Einheit eigentlich erst verstanden werden kann durch Rekurs auf die Katergorien als Vorstellungsarten des ursprünglichen Vorstellens. Beck erläutert die Zurückführung der analytischen Einheit des dynamisch erklärten Begriffs der Materie auf die ursprüngliche Synthesis folgendermaßen:

"Indem mein Finger in einen Raum einzudringen bestrebt ist und Widerstand erfährt, so ist es die
ursprüngliche Synthesis meiner Empfindung und die ursprüngliche Zeitbestimmung dieser
Empfindung, die diesem Begriffe von Erfüllung des Raums unterliegt."

Wie in 8.3. dargelegt, besteht für Beck das empirische Anschauen in der "ursprüngliche[n] Synthesis des Gleichartigen"74. Diese Synthesis bedarf jedoch noch, wie aus dem Text hervorgeht, einer "ursprünglichen Zeitbestimmung dieser Empfindung", ohne die sich die Erfüllung des Raums nicht verstehen lasse. ${ }^{75}$ Beck fährt fort:

\footnotetext{
"Indem [...] mein Finger in einen gewissen Raum zu dringen strebt und einen Widerstand erfährt, so besteht die Erzeugung der ursprünglich-synthetischen Einheit der Erfahrung eben dieses Widerstandes in der ursprünglichen Synthesis und der Bestimmung dieser Synthesis meiner Empfindung, die mit dem ursprünglichen Setzen eines Etwas, worauf die Empfindung folgen mußte, verbunden ist; das heißt: das Reale, das den Raum erfüllt, ist bewegende Kraft." ${ }^{76}$
}

Die Zeitbestimmung der Synthesis der Empfindung macht nun die Annahme des "ursprünglichen Setzens eines Etwas, worauf die Empfindung folgen mußte", erforderlich. Beck kann nicht umhin, hervorzuheben, daß die ursprüngliche Synthesis des Gleichartigen "ein Anschauen" und als solches "von dem ursprünglichen Setzen in der Kategorie der Causalität verschieden" sei. Diese Feststellung widerspricht nicht der These Becks, "daß das Anschauen [...] mit der Kategorie der Größe [...] einerley ist."77 Beide als mathematische und dynamische Kategorienklassen unterschiedene Arten der Synthesis sind offenbar auch terminologisch so zu trennen, daß das Setzen von Expansiv- und Attraktionskräften eben nicht als Anschauen, doch als ein ursprüngliches Vorstellen bezeichnet werden kann. ${ }^{78}$ Es ist auffällig, daß Beck ausdrücklich darauf hinweist, daß das Konstruieren von dem ursprünglichen Vorstellen zu unterscheiden sei: "den Begriff der Materie zu construiren" sei "eine rein-mathematische Aufgabe", während das

73 Beck (1796) 211.

74 Ebd. 367.

75 Beck (1796) 145 führt aus, daß "meine Empfindung" in der ursprünglichen Synthesis "synthesirt" werde: "In dieser Synthesis [...] erzeuge ich die Zeit. Das Bestimmen dieser Zeit, wodurch diese Synthesis selbst fixirt wird, ist die ursprüngliche Anerkennung. In diesem ursprünglichen Vorstellen wird [...] der Begriff, wodurch ich das Reale eines Dinges denke, erzeugt." - Becks Einführung des Zeitbegriffs, wonach "mir" in der Synthesis die Zeit "entsteht" (143, vgl. 151), ist nicht überzeugend; Heller (1992) 94 spricht von einer ad hoc-Hypothese.

76 Beck (1976) 213.

77 Ebd. 367.

78 Ebd. 217: "In eben demselben ursprünglichen Vorstellen aber, worin der Verstand objectiv-synthetische Einheit des Bewußtseyns erzeugt, indem er ursprünglich-expansive Kraft setzt, welche das Reale der Materie, ihre Substanz, kurz, die Materie selbst ist, limitirt auch der Verstand diese ursprüngliche Erfüllung des Raums, in welchem ursprünglichen Vorstellen die Bedeutung des Begriffs von einer, einen bestimmten Raum einnehmenden Materie liegt." 
ursprüngliche Vorstellen diesem Begriff erst seine "Bedeutung gebe". ${ }^{79}$ Unmißverständlich heißt es dazu:

"Da war nun das ursprüngliche Setzen des beharrlichen Realen, der Expansiv-Kraft, und die ursprüngliche Limitation derselben, nicht eine ursprüngliche Synthesis des Gleichartigen, das heißt: es war dasselbe keine Construction."

Als wolle er diese Klarstellung zurücknehmen, setzt Beck die Passage jedoch so fort:

"Das ursprüngliche Vorstellen aber, worauf sich der Begriff des Conflictus beyder Kräfte, wodurch ein bestimmter Raum erfüllt ist, stützt, ist eine Construction." ${ }^{80}$

Es liegt die Vermutung nahe, daß Beck mit diesem Satz, in dem die Gleichsetzung der Begriffe 'ursprüngliches Vorstellen' und 'Konstruktion' in der Erläuterung des Kantischen Materiemodells zweier konfligierender Kräfte vollzogen wird, den entscheidenden Impuls zur Transformation des Konstruktionsbegriffs gibt.

\subsection{Schellings Interpretation des Konstruktionsb egriffs in Kants MANW (1797)}

Es wäre weit gefehlt, wollte man die Transformation des Konstruktionsbegriffs vom mathematischen zum philosophischen Methodenbegriff bloß auf die Ersetzung des Begriffs des ursprünglichen Vorstellens durch den Begriff der Konstruktion reduzieren. Diese Ersetzung hätte dann die womöglich mißverständliche Ausdrucksweise Becks veranlaßt. Die Transformation des Begriffs vollzieht sich vielmehr vor dem Hintergrund eines präzise bestimmbaren Problemfeldes. Es geht um die Frage, welche philosophische Interpretation dem Konstruktionsbegriff in den $M A N W \mathrm{zu}$ geben ist,

(a) sofern diese Interpretation sich auf die Funktion der MANW als "Probe" und "Realisierung" der Transzendentalphilosophie bezieht. Damit verbunden ist die Aufklärung des Status des Empfindungsbegriffs in Kants transzendentaler Theorie der Erfahrung im Hinblick auf die Begriffsbestimmungen 'rein', 'empirisch', 'a priori' und 'a posteriori'.

(b) sofern mit dieser Interpretation Funktion und Grenzen philosophischer und mathematischer Methodik in der Perspektive ihrer Vermittlung in der Naturwissenschaft bestimmt und in der Folge offenbar übereinandergeschoben werden können. Den MANW wuchs damit die Bedeutung zu, ein Modell philosophischer Letztbegründung vorzuführen. Die Analyse ihrer Resultate bildete die Folie, auf der die unentschiedene Prämissenfrage des Kantischen Systems gelöst werden sollte. Kants Begründungsversuch der mathematischen Konstruktion des dynamischen Begriffs der Materie ${ }^{81}$ konnte so geradezu als "Modifikation" und "Beispiel" philosophischer Konstruktion aufgefaßt werden. ${ }^{82}$

79 Ebd. 219.

80 Ebd. 219; vgl. 210, 277.

81 Vgl. Kants "kleine Vorerinnerung zum Behufe des Versuchs einer solchen vielleicht möglichen Konstruktion" in MADyn: AA IV 518-523 = WW V 76-82.

82 Vgl. Höijer (1801) 166f. 
Beck sah die Aufgabe der MADyn auf "jene allgemeine Anzeige beyder ursprünglichen Kräfte und ihrer Vereinigung zum Behufe der Erfüllung eines bestimmten Raums" begrenzt. ${ }^{83}$ Für Schelling läßt sich an Kants dynamischer Erklärung des Begriffs der Materie eine Begründungsstruktur ganz eigener Art aufzeigen. Er verweist dabei auf die "Trichotomie in allen Eintheilungen der TransscendentalPhilosophie", die Kant auf das zurückführe, "was zu der synthetischen Einheit überhaupt erfoderlich ist, nämlich 1) Bedingung 2) ein Bedingtes, 3) der Begriff der aus der Vereinigung des Bedingten mit einer Bedingung entspringt". ${ }^{84}$ Dieses Bedingungsgefüge werde "durch den Entwurf der ursprünglichen VerstandesForm, (die Tafel der Kategorieen) anschaulich gemacht." Schelling versucht am Beispiel der Kategorien der Qualität ${ }^{85}$ zu zeigen, daß weder Realität noch Negation absolut, sondern beide "nur in einem dritten vorstellbar [sind], das durch ihre Vereinigung entspringt. [...] Beide sind nur in ihrer Vereinigung vorstellbar" ${ }^{86}$

\footnotetext{
"Denken wir uns etwa, daß der Raum lediglich durch repulsive Kraft (ohne entgegenstehende Kraft) ausgefüllt wäre, so wird die Materie unendlich ausgedehnt, (nichts als Porus) d.h. der Raum wird leer seyn. Denken wir uns umgekehrt die AttractivKraft als absolut, so wird alle Materie in einem (mathematischen) Punkt vereinigt (absolut dicht) d.h. der Raum wird abermals leer seyn." ${ }^{87}$
}

Da die Raumerfüllung erst durch die Vereinigung beider sich einschränkender Kräfte vorstellbar werde, sei die dritte Kategorie der Limitation "nirgends als eine leere Form a priori $d a "$. Limitation sei jedoch auch keine bloße Vorstellungsart der ursprünglichen Synthesis, wie Beck annahm, sondern werde "erst thätig erzeugt [...] durch ein Thun, in welchem ebendesswegen Realität und Negation ursprünglich absolut vereinigt seyn müssen." 88 Anhand dieses Modells der Einheit in der Duplizität von Bedingung und Bedingtem formuliert Schelling seine Kritik an Beck nun so, daß er den Begriff der Konstruktion als Interpretament des Theorems des ursprünglichen Vorstellens einsetzt: "diese nothwendige Vereinigung Entgegengesetzter in unserm Construiren können wir uns gar nicht, als ursprünglich vorstellen [...], ohne vorauszusetzen, daß in uns ursprünglich das Bedingende und das Bedingte, das Bestimmende und das Bestimmte [...] ABSOLUTIDENTISCH seyen". ${ }^{89}$

Schelling legt sich die Frage vor, unter welcher Voraussetzung "Vereinigung Entgegengesetzter in unserm Construiren [...] als ursprünglich vorstellen" aufgefaßt werden kann. Nun besteht Konstruktion in der Vereinigung entgegengesetzter Momente, die sich in der Entgegensetzung wie Bedingung und Bedingtes zueinander verhalten. Die in der Konstruktion vollzogene Vereinigung reproduziert die ursprüngliche Identität der Momente, deren begrifflich vermittelte Entgegensetzung in dieser Identität ihren Bezugspunkt hat. Werden Konstruktion und Vorstellung als Tätigkeiten begriffen, deren

83 Beck (1796) $219 \mathrm{f}$.

84 Übersicht: SW I 425 = AW IV 152. Schelling zitiert aus der "Einleitung" der $K U:$ AA V 197* =WW V $273^{*}$.

85 KrV A80 B106: "Tafel der Kategorien [...] 2. Der Qualität: Realität / Negation / Limitation. [...]". P $\$ 21$ : AA IV 303 = WW III 169: "Transzendentale Tafel der Verstandesbegriffe [...] 2. Der Qualität Realität / Negation / Einschränkung [...]".

86 Übersicht: SW I 425f. = AW IV 152f.

87 Ebd.: SW I 425f. = AW IV 152.

88 Ebd.: SW I 426 = AW IV 153.

89 Ebd.: SW I 426f. = AW IV 153. 
intentionaler Gegenstand die Vereinigung entgegengesetzter Momente ist, müssen diese Tätigkeiten auf ein Prinzip zurückgeführt werden können, das die Duplizität in der Tätigkeit des Vereinigens begründet. Schelling greift auf dieses Modell auch in den Ideen zu einer Philosophie der Natur (1797) zurück, die ungefähr gleichzeitig mit den in der Übersicht gesammelten Beiträge für das Philosophische Journal entstanden sind..$^{90}$ Besonders aufschlußreich für das Verständnis des transformierten Konstruktionsbegriffs ist die Interpretation des Kantischen Materiemodells zweier konfligierender Kräfte im zweiten Buch der Ideen von 1797 gerade deshalb, weil Schelling hier den Begriff der in der Übersicht "ursprünglich (transscendental)" genannten Konstruktion noch gar nicht verwendet.

Erst im nachhinein, in den "Zusätzen" der zweiten Auflage der Ideen von 1803, wird Schelling von der "Konstruktion der Materie" da sprechen, wo es ihm 1797 um den "Ersten Ursprung des Begriffs der Materie aus der Natur der Anschauung und des menschlichen Geistes" geht. ${ }^{91}$ Dementsprechend ergänzt Schelling das fünfte Kapitel, das "Grundsätze der Dynamik" überschrieben ist, 1803 um "Anmerkungen über die voranstehende idealistische Konstruktion der Materie". ${ }^{92}$ Wo 1797 der transformierte Konstruktionsbegriff noch gar nicht auftritt, so läßt sich doch anhand des Problemfeldes, für das 1803 der Konstruktionsbegriff einstehen soll, genau die Fragestellung bestimmen, deren Lösung Schelling die Transformation des Konstruktionsbegriffs dringlich erscheinen läßt. Schelling geht es hierbei um die Frage, wie der dynamisch interpretierte Begriff der Materie mathematisch konstruiert werden kann. Es ist diese Frage, an der sich das Konstruktionsproblem in den $M A N W$ festmachen läßt und deren Lösungsversuch die KantForschung im Anschluß an Plaaß (1965) zur Bildung des Problemtitels der metaphysischen Konstruktion veranlaßt hat. ${ }^{93} \mathrm{Im}$ folgenden werde ich Schellings Analyse des Konstruktionsproblems in den MANW kurz vorstellen. Aus der Beschreibung des Gesichtspunktes, von dem aus der Konstruktionsbegriff in den MANW für Schelling überhaupt zu einem Problem werden konnte, lassen sich Ansatz und Ziel der Transformation des Konstruktionsbegriffs einsichtig machen.

Zum methodischen Ansatz seiner Interpretation des Kantischen Materiemodells führt Schelling 1797 aus:

\footnotetext{
"Kräfte sind doch einmal nichts, das in der Anschauung darstellbar ist. Gleichwohl verläßt man sich auf jene Begriffe von allgemeiner Anziehung und Zurückstoßung so sehr, setzt sie überall so offenbar und bestimmt voraus, daß man von selbst auf den Gedanken geräth, sie müssen, wenn sie nicht selbst Gegenstände möglicher Anschauung, doch Bedingungen der Möglichkeit aller objektiven Erkenntniß seyn. [...] so müssen wir zum voraus vermuthen, daß ihr Ursprung unter den Bedingungen der menschlichen Erkenntniß überhaupt zu suchen ist, und insofern wird unsere Untersuchung eine transscendentale Erörterung des Begriffs von einer Materie überhaupt seyn. Hier sind nun zweierlei Wege möglich. Entweder man analysirt den Begriff der Materie selbst und zeigt etwa, daß sie überhaupt gedacht werden muß als etwas, das den Raum, jedoch unter bestimmten Schranken, erfüllt, daß wir also als Bedingung ihrer Möglichkeit voraussetzen müssen eine Kraft, die den Raum erfüllt, und eine andere jener entgegengesetzte, die dem Raum Grenze und Schranke gibt." ${ }^{94}$
}

$90 \quad$ Vgl. Jacobs (1988) 4.

91 Ideen: SW II 213, 223.

92 Ideen: SW II 227, 239.

93 Vgl. Kap. 2 und 3.

94 Ideen: SW II $213 \mathrm{f}$. 
Die "transscendentale Erörterung des Begriffs von einer Materie überhaupt" läßt "zweierlei Wege" zu, deren ersten Schelling im zitierten Textstück als Begriffsanalyse kennzeichnet. Dieses analytische Verfahren besteht darin, die Bedingungen der Möglichkeit von Raumerfüllung aufzuweisen. Mit dieser These gehört Schelling im Anschluß an Beck wohl zu einem der ersten und bis heute wenigen Interpreten, die Kants $M A N W$ einen transzendentalen Ansatz zugestehen. ${ }^{95}$

Nach dem in der Übersicht entworfenen Modell der Einheit in der Duplizität entgegengesetzter Momente, begrifflich als Bedingung und Bedingtes vermittelt, bestimmt Schelling dann mit Kant die Repulsion als positive Kraft, der die Attraktion als negative Kraft entgegengesetzt ist:

\footnotetext{
"Daraus ist klar, daß, weil das Negative überhaupt in logischer Bedeutung nichts an sich selbst ist, sondern nur die Verneinung des Positiven ist [...], die Zurückstoßungskraft der Anziehungskraft logisch vorangehen muß. Allein die Frage ist, welche von beiden der andern in der Wirklichkeit vorangehe [...]: keine von beiden; jede einzelne ist nur da, insofern ihre entgegengesetzte da ist, d.h. sie sind selbst in Bezug aufeinander wechselseitig positiv und negativ, jede einzelne beschränkt nothwendig die Wirkung der andern, und nur dadurch werden sie ursprüngliche Kräfte einer Materie." 96
}

Gegen die analytische Variante der transzendentalen Erörterung wendet Schelling jedoch ein, daß man auf diese Weise "verführt" werde, den Begriff "als einen willkürlichen, selbstgemachten Begriff zu betrachten, so daß ihm am Ende keine andere, als bloß logische Bedeutung übrig bleibt." "Sicherer also" sei es, "man läßt den Begriff vor seinen Augen gleichsam entstehen, und findet in seinem Ursprung selbst den Grund seiner Nothwendigkeit. Dieß ist das synthetische Verfahren." ${ }^{97}$ Es ist die nun folgende weitläufige Darstellung dieses synthetischen Verfahrens, in dem Schelling die Ursprungserklärung des Begriffs der Materie "aus der Natur der Anschauung und des menschlichen Geistes" im einzelnen nachzeichnet, und zwar bis zu dem Punkt, "wo der Begriff von Materie einer analytischen Behandlung fähig wird, und die Grundsätze der Dynamik aus diesem Begriffe allein mit Fug und Recht abgeleitet werden können. Dieses Geschäft aber ist in Kants metaphysischen Anfangsgründen der Naturwissenschaft mit einer solchen Evidenz und Vollständigkeit geschehen, daß hier nichts weiter zu leisten übrig ist." 98

Von Bedeutung ist nun, daß Schelling in der Fassung der Ideen von 1797 noch ohne Konstruktionsbegriff da auskommt, wo er 1803 explizit von Konstruktion spricht, selbst im fünften Kapitel, das Kants analytisch genannte Herleitung der Grundsätze der Dynamik ohne Vorbehalte wiedergibt. Diese Beobachtung deckt sich mit der heuristischen These, die Schelling im Anschluß an Höijer äußert, "daß Kant, wenn er ein vollendetes Bewußtseyn seiner Philosophie hätte und der Reflexion darüber fähig wäre, construiren müßte". ${ }^{99}$

95 Vgl. die in 5.6. vorgetragene Interpretation des Konflikts zweier Grundkräfte als transzendentale Synthesis empirischer Anschauung.

96

Ideen: SW II $232 \mathrm{f}$.

Ideen: SW II 214.

98 Ideen: SW II 231.

99 Konstruktion: SW V 140. 
In den Ideen von 1797 allerdings tritt der Konstruktionsbegriff nur im letzten Kapitel auf, das den Titel trägt: Versuch über die ersten Grundsätze der Chemie. Es gehe ihm darum, so schreibt Schelling mit Verweis auf Eschenmayer, "die Principien der Dynamik, sowie sie von Kant aufgestellt sind, [...] auf empirische Naturlehre, vorzüglich Chemie, anzuwenden." ${ }^{100}$ Chemie, sofern sie die Qualitäten der Materie zum Gegenstand hat, betrachtet Schelling als "angewandte Dynamik". Damit könnte die spezifische Verschiedenheit der Qualitäten auf die unterschiedlichen Intensitätsgrade der Grundkräfte zurückgeführt werden und, - vorausgesetzt, "Begriffe, die sich auf Grade überhaupt beziehen, wie Qualität, Kraft u.s.w.", wären in der "Anschauung a priori darstellbar" mathematisch konstruiert werden. Schelling kommt im Abschnitt "Konstruktion der chemischen Bewegungen" jedoch noch $\mathrm{zu}$ dem Schluß, daß die mathematische Konstruktion nicht möglich ist. ${ }^{101}$

Daß es Schelling im zweiten Buch der Ideen von 1797 zunächst um die Frage geht, ob und wie der dynamisch interpretierte Begriff der Materie mathematisch konstruierbar ist, liegt auf der Hand. Um Ansatz und Ziel der Transformation des Konstruktionsbegriffs zu verdeutlichen, bleibt aufzuklären, welche Bedeutung das Problem der mathematischen Konstruktion in den $M A N W$ für die in der frühidealistischen Debatte aufgeworfene Frage nach den Grundlagen der Transzendentalphilosophie Kants gewinnt. In den Augen Schellings steht der Nachweis der mathematischen Konstruierbarkeit des dynamischen Materiebegriffs für zweierlei ein: Chemie, verstanden als "angewandte Dynamik", wäre mit dem Nachweis nach den Prinzipien der dynamischen Naturphilosophie Kants Vorgaben gemäß "einer wissenschaftlichen Darstellung" fähig. ${ }^{102}$ Darüber hinaus kann erst durch die Konstruktion der Qualitäten, d.h. der spezifischen Verschiedenheit der Materie, eigentlich begriffen werden, daß und inwiefern ein Zusammenhang zwischen dem Auftreten von Empfindungen in uns als sinnlichen Eindrücken und ihrer Interpretation durch Begriffe, die Qualitäten bezeichnen, gerechtfertigterweise behauptet wird.

\footnotetext{
"Alles, was zur Qualität der Körper gehört, ist bloß in unserer Empfindung vorhanden, und was empfunden wird, läßt sich niemals objektiv (durch Begriffe), sondern nur durch Berufung auf das allgemeine Gefühl verständlich machen. Allein damit ist nicht aufgehoben, daß das, was in einer Rücksicht Gegenstand der Empfindung ist, in anderer Rücksicht auch Objekt für den Verstand werden könne." 103
}

Diesen "Widerstreit", daß, was "Gegenstand der Empfindung" sei, doch "Objekt für den Verstand " werden könne, sieht Schelling "nur in dem Gesichtspunkt" begründet, von dem

100 Ideen: SW II 313.

101 Ideen: SW II 334-337. "[...] jede Bewegung ist nur relativ vorstellbar, und insofern auch (nach phoronomischen Grundsätzen) konstruirbar. Wenn man fragt, ob chemische Bewegung, als solche, konstruirt werden könne, so heißt dieß so viel: ob die chemischen Bewegungen, wechselsweise aufeinander (nicht etwa auf einen Körper, der nicht in den chemischen Proceß fällt) bezogen, konstruirt werden können? Wird die Frage so ausgedrückt, so sieht man sogleich ein, daß sie verneint werden muß - denn chemische Bewegungen, als solche, bestimmen keinen materiellen Raum, auf den ich sie beziehen könnte. Dieser materielle Raum ist selbst erst Resultat der chemischen Bewegung, d.h. er wird nicht phoronomisch - beschrieben, sondern dynamisch (durch Wechselwirkung von Kräften) erzeugt." (337; vgl. 316) - Moiso (1985) verfolgt die Entwicklung von "Schellings Elektrizitätslehre 1797-1799" bis zu dem Punkt, wo "eine vollständige Konstruktion der Materie" (94) als möglich erscheint.

102 Ideen: SW II $316 f$.

103 Ideen: SW II 267. 
aus Empfindung und Begriff der Empfindung betrachtet werden. ${ }^{104}$ Den Zusammenhang aufzuzeigen, der die Einheit zwischen den vermeintlich widerstreitenden Gesichtspunkten stiftet, dient nun das von Schelling im vierten Kapitel des zweiten Buches der Ideen von 1797 eingeführte "synthetische Verfahren" der transzendentalen Erörterung des Begriffs von einer Materie überhaupt. ${ }^{105}$

Dem Verfahren, das hier nur kurz skizziert werden soll, liegt die Absicht zugrunde, das Materiemodell zweier konfligierender Kräfte dem durch die empirische Anschauung vermittelten Eindruck, der Empfindung, als strukturanalog zu erweisen. ${ }^{106}$ Dabei faßt Schelling die "Natur der Anschauung" im Rekurs auf Fichtes Theorem der Anschauung als Ausdruck zweier entgegengesetzter Tätigkeiten auf. ${ }^{107}$ So könne der "Ursprung des Begriffs der Materie" auf die duale Struktur der Anschauung zurückgeführt werden. Sofern wir empirische Anschauung von äußeren Dingen haben, betrachten wir das, was wir an ihnen durch Empfindung gewahren und als materiale Eigenschaft, z.B. Undurchdringlichkeit, bezeichnen, als notwendig. Nun können wir uns die Eigenschaft der Undurchdringlichkeit durch das Modell des Zusammentreffens zweier Kräfte begreiflich machen. Daher gehöre zur Möglichkeit eines Gegenstandes überhaupt nichts weiter als ein Zusammentreffen dynamischer Kräfte, wodurch ein unbestimmtes Objekt als bloßer Begriff gedacht werde könne.

Was die Allgemeinheit des Begriffs und damit die Möglichkeit des Gegenstandes überhaupt ausmacht, gilt uns als notwendig, was dessen spezifische Bestimmtheit ausmacht, als zufällig. Die Funktion des mathematischen Konstruktionsbegriffs in dieser Deduktion erhellt nun aus der Beschreibung des unbestimmten, doch im Urteilsakt bestimmbaren Gegenstandes:

"Jenes unbestimmte Etwas, das Nothwendige in allen unsern Vorstellungen von einzelnen Dingen, ist [...] eine Sphäre, eine Quantität, überhaupt etwas, was bloß denkbar oder konstruirbar ist." 108

Die Formel: "bloß denkbar oder konstruirbar" zeigt zweierlei an: Unter demjenigen, "was bloß denkbar oder konstruirbar ist", versteht man ein mögliches Objekt des Denkens oder der Konstruktion. Hier greift Schelling offensichtlich auf die Bestimmung des Prinzips der Philosophie in der Abhandlung Vom Ich zurück: "Dieses als das Einige Unbedingbare [...] beschreibt die Sphäre alles Denkbaren"109. In den Ideen von 1797 wird dieses Prinzip wie in der Übersicht als ursprüngliche Tätigkeit expliziert:

"Allem Denken und Vorstellen in uns geht also nothwendig voran eine ursprüngliche Thätigkeit, die, weil sie allem Denken vorangeht, insofern schlechthin - unbestimmt und unbeschränkt ist. Erst nachdem ein Entgegengesetztes da ist, wird sie beschränkte, und eben deßwegen bestimmte (denkbare) Thätigkeit." ${ }^{110}$

${ }^{104}$ Ideen: SW II 267.

${ }^{105}$ Ideen: SW II $213 \mathrm{ff}$.

106 Ideen: SW II 213ff., 267-272.

107 Vgl. Löw (1979), Moiso (1985).

${ }^{108}$ Ideen: SW II $269 \mathrm{f}$.

${ }^{109}$ Vom Ich: SW I $176=$ AW II 100. Vgl. 8.2.

${ }^{110}$ Ideen: SW II 218. Vgl. 217: "die ursprüngliche Thätigkeit in mir [wird] erst am Objekte zum Denken, zum selbstbewußten Vorstellen." 
Nun ist in der Formel: "was bloß denkbar oder konstruirbar ist" nicht nur auf einen diesem möglichen Objekt des Denkens oder Konstruierens und dem Akt des Gedachtwerdens oder des Konstruiertwerdens vorgängigen Einheitsgrund, verstanden als Tätigkeit, verwiesen. Die Formel macht darüber hinaus deutlich, daß die Alternative "denkbar oder konstruirbar" keine sich ausschließende, sondern explikative Beziehung zwischen beiden Operationen bezeichnet. Daß dasjenige, was gedacht, auch konstruiert werden kann, heißt, daß auch aus der Sicht Schellings die mathematische Konstruktion der Begriffe integraler Bestandteil objektiver Erkenntnis ist.

Wie sich im folgenden Kapitel zeigen wird, kann Schelling mit dieser These einerseits auf Jacobis scharfsinnige wissenschaftskritische Diagnose des Kantischen Konstruktionstheorems zurückgreifen, "daß wir unmöglich begreifen können, was wir zu construiren nicht im Stande sind". ${ }^{111}$ Andererseits verteidigt Schelling dieses Theorem gegen Jacobis vernunftkritische Einstellung, wonach jede Form begrifflich vermittelter Demonstration aporetisch verlaufe. Es ist die durch Beck vermittelte Neuinterpretation des Kantischen Konstruktionsbegriffs, die ihm die Mittel für die Transformation des Konstruktionsbegriffs und seine Verteidigung gegen antiintellektualistische Einwände an die Hand gibt. Konstruktion soll dabei offenbar keine genetisch verfaßten Bedingungsverhältnisse, sondern die Zurückführung solcher Verhältnisse auf die Identität dessen beschreiben, was in uns nur in einer begrifflich vermittelten Duplizität entgegengesetzter Momente vorstellbar wird. Schelling zufolge charakterisiert diese Identität das Prinzip des transzendentalen Idealismus, mit dem wir uns am Beispiel mathematischer Konstruktion vertraut machen.

"Diese ursprüngliche Identität des Reinen und Empirischen in uns ist nun eigentlich das Princip alles transscendentalen Idealismus. Aus diesem Princip erst erklärt sich, warum in uns ursprünglich zwischen Realem und Idealem, zwischen dem, was empfunden, und dem was gehandelt wird, zwischen dem, was wir (vom Standpunkt des Bewusstseyns aus) a priori und a posteriori nennen, [...] zwischen Anschauung und Begriff, gar kein Unterschied statt finde?" ${ }^{112}$

Es ist abschließend zu klären, inwiefern Schelling im System des transzendentalen Idealismus (1800) gerechtfertigterweise von der Selbstkonstruktion dieses Prinzips sprechen kann.

111 Jacobi (1789) 420*. Vgl. 9.2.

112 Übersicht: SW I 427 = AW IV 153 f. 


\section{Selbstkonstruktion als Prinzip der Transzendentalphilosophie}

In diesem Kapitel sollen die Argumentationsstränge und Belege zur Interpretation des Konstruktionstheorems zusammengeführt werden. Wie in 8.3.-8.6. dargelegt, erschöpft sich Schellings Einführung des Konstruktionsbegriffs als eines philosophischen Methodenbegriffs nicht in der Ersetzung des Begriffs des ursprünglichen Vorstellens, zu der Schelling zudem nur durch die schillernde Ausdrucksweise Becks bei der Erläuterung des Kantischen Konstruktionstheorems inspiriert worden wäre. Es gibt zwei Gründe, die Schelling die Transformation des Begriffs dringlich erscheinen ließen: Einerseits erforderte der Anspruch, in Kants MANW ein Modell philosophischer Letztbegründung zu sehen, auch eine Neuinterpretation des Kantischen Konstruktionsbegriffs. Andererseits sind es die methodologischen Merkmale 'geometrischer Beweisart' und ihre - wiederum durch Beck angeregte - philosophische Interpretation, die das Verfahren der Konstruktion, verstanden als Demonstration, der philosophischen Methodologie selbst verfügbar machen sollten.

\subsection{Konstruktion als Demonstration}

Am Beispiel der MADyn benennt Beck deutlich Aufgabe und Grenzen metaphysischer Deduktion. Aufgabe der $M A N W$ sei "jene allgemeine Anzeige beyder ursprünglichen Kräfte und ihrer Vereinigung zum Behufe der Erfüllung eines bestimmten Raums". ${ }^{1}$ Schon Kant weist in den $M A N W$ wiederholt auf die bloß heuristische Funktion der Metaphysik im Bereich der Naturforschung hin. Die Aufgabe der Naturphilosophie bestehe "in der Zurückführung gegebener, dem Anscheine nach verschiedener, Kräfte auf eine geringere Zahl Kräfte und Vermögen [...], welche Reduktion aber nur bis zu den Grundkräften fortgeht, über die unsere Vernunft nicht hinaus kann."2 Aufgabe der Metaphysik sei es,

\footnotetext{
"die Naturphilosophie, so weit, als es immer möglich ist, auf die Erklärung der dynamischen Erklärungsgründe zu leiten [...]. Dies ist nun alles, was Metaphysik zur Konstruktion des Begriffs der Materie, mithin zum Behuf der Anwendung der Mathematik auf Naturwissenschaft, in Ansehung der Eigenschaften, wodurch Materie einen Raum in bestimmtem Maße erfüllet, nur immer leisten kann, nämlich diese Eigenschaften als dynamisch anzusehen und nicht als unbedingte ursprüngliche Positionen, wie sie etwan eine bloß mathematische Behandlung postulieren würde." ${ }^{3}$
}

Diese eher heuristisch angelegte Funktion der Metaphysik bei der Begründung mathematischer Naturwissenschaft will Kant entschieden von der bloß mathematischen Behandlungsweise abgegrenzt wissen. Deren Vorgehen besteht darin, die Eigenschaften der Materie "als unbedingte ursprüngliche Positionen" zu "postulieren". Kant nennt "Lambert und andere", die die Eigenschaft der Materie, einen Raum zu erfüllen, schlicht

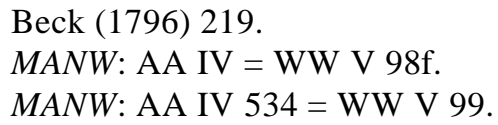


als "Solidität" deuten, und zwar in der Absicht, die Raumerfüllung durch einen nicht weiter ableitbaren Grundbegriff zu erklären. Kants Einwand lautet:

\footnotetext{
"Hier hat der Mathematiker etwas als ein erstes Datum der Konstruktion des Begriffs einer Materie, welches sich selbst weiter nicht konstruieren lasse, angenommen. Nun kann er zwar von jedem beliebigen Dato seine Konstruktion eines Begriffs anfangen, ohne sich darauf einzulassen, dieses Datum auch wiederum zu erklären; darum aber ist er doch nicht befugt, jenes für etwas aller mathematischen Konstruktion ganz Unfähiges zu erklären, um dadurch das Zurückgehen zu den ersten Prinzipien in der Naturwissenschaft zu hemmen." ${ }^{4}$
}

Die Konstruktion beginnt mit dem Datum, hier von Kant als "ein erstes Datum der Konstruktion" eingeführt. ${ }^{5}$ Das Datum ist insofern beliebig gewählt, als der Mathematiker das Auftreten bzw. die Existenz des Datums nicht weiter erklären müsse. Das Datum zu erklären, heißt für Kant offenbar, es nicht als "etwas aller mathematischen Konstruktion ganz Unfähiges" aufzufassen. Mit der Konstruierbarkeit sieht er zugleich einen Schritt im "Zurückgehen $\mathrm{zu}$ den ersten Prinzipien der Naturwissenschaft" getan. Mit dieser Feststellung erinnert Kant an seine These, daß die mathematische Konstruierbarkeit von Begriffen ein integraler Bestandteil der Erkenntnis objektiver Sachverhalte ist, die mit Hilfe solcher Begriffe naturwissenschaftlich beschrieben werden können. Nun verfügt Kant auch über eine Lesart des Konstruktionsbegriffs, nach der Konstruktion als Demonstration und Demonstration als ein der transzendentalen Theorie der Erfahrung immanentes Beweisverfahren begriffen werden kann:

\footnotetext{
"Verstandesbegriffe müssen, als solche, jederzeit demonstrabel sein (wenn unter Demonstrieren, wie in der Anatomie, bloß das Darstellen verstanden wird); d.i. der ihnen korrespondierende Gegenstand muß jederzeit in der Anschauung (reinen oder empirischen) gegeben werden können: denn dadurch allein können sie Erkenntnisse werden. Der Begriff der Größe kann in der Raumesanschauung a priori, z.B. einer geraden Linie u.s.w., gegeben werden. Der Begriff der Ursache an der Undurchdringlichkeit, dem Stoße, der Körper u.s.w. Mithin können beide durch eine empirische Anschauung belegt, d.i. der Gedanke davon an einem Beispiele gewiesen (demonstriert, aufgezeigt) werden; und dieses muß geschehen können: widrigenfalls man nicht gewiß ist, ob der Gedanke nicht leer, d.i. ohne alles Objekt sei."6
}

Kant betrachtet Demonstration als philosophisches Verfahren, die objektive Realität derjenigen Begriffe zu erweisen, die konstitutive Bedingungen der Möglichkeit von Erfahrungserkenntnis sind. Dabei können "beide" Verstandesbegriffe der mathematischen wie dynamischen Kategorienklassen (hier: Größe und Ursache) "durch eine empirische Anschauung belegt" werden. Für den Fall, daß der Begriff schon in der Anschauung a priori gegeben werden kann, spricht Kant von der Konstruktion des Begriffs. In der Form der Demonstration, nämlich durch "Beispiele (Fälle in concreto) [...] die Begriffe und Lehrsätze [...] der Transzendentalphilosophie $\mathrm{zu}$ realisieren, d.i. einer bloßen Gedankenform Sinn und Bedeutung unterzulegen", sieht Kant genau die Aufgabe der $M A N W .^{7}$

MANW: AA VI $498=$ WW V 49.

Zur Gliederung des euklidischen Beweisverfahrens in sechs Schritte und zu Kants Interpretation dieses Verfahrens vgl. 4.2. und 4.3.

$K U \S 57$ Anm.I: AA V $240=$ WW V 448.

$M A N W$ : AA IV $478=\mathrm{WW}$ V $23 \mathrm{f}$. 
Auch Beck ist der Meinung, daß die Kompetenz der Metaphysik da endet, "wo die Construction der Begriffe beginnt". ${ }^{8}$ Und schon in seinem ersten Kommentar zu den $M A N W$, vor der Konzeption der Theorie des ursprünglichen Vorstellens, beschreibt er diese Aufgabenbegrenzung noch begriffsspezifischer als "Exposition der Data zur Construction des Begriffs der Materie, welche die Philosophie der Mathematik überliefert". ${ }^{9}$ Nun hat Kant mit der Formulierung des Konstruktionstheorems eine philosophische Interpretation der Mathematik in der Perspektive ihrer Anwendung auf Naturwissenschaft gegeben. Beck muß eben diese Perspektive vor Augen gehabt haben, wenn er Kants Definition der Mathematik gegen den "Einwurf" verteidigt,

"daß die Critik in die [sic] Definition der Mathematik eigentlich die Methode derselben beschrieben habe, da sie doch eigentlich das Objekt dieser Wissenschaft anzeigen sollte. Die Größe ist der Gegenstand derselben [...]. Da druckt bey weitem treffender das Merkmahl: Construction der Begriffe, den Geist dieser Wissenschaft aus, wodurch auf den Sinn des Begriffs: Größe, und darauf gewiesen wird, daß die Begriffe in der Mathematik auf diese ursprüngliche Synthesis des Gleichartigen zurück geführt werden." ${ }^{10}$

Ein Datum zu erklären, heißt für Kant, dessen Konstruierbarkeit zu erweisen und damit das Zurückgehen zu ersten Prinzipien zu fördern. Nun interpretiert Beck auf der Folie seiner Theorie des ursprünglichen Vorstellens Konstruktion eben als Zurückführen des Begriffs der Größe auf die "ursprüngliche Synthesis des Gleichartigen". Danach könne der Begriff der Größe, als Begriff, erst "Bedeutung und Verständlichkeit" haben, wenn seine analytische Einheit durch "die Construction [...] in der Zurückführung desselben auf die ursprüngliche Synthesis des Gleichartigen, welche synthetische Einheit des Bewußtseyns erzeugt", verstanden werde. ${ }^{11}$ Durch die philosophische Interpretation der geometrischen Konstruktion wird die Funktion, die Konstruktion als ein Schritt im euklidischen Beweisverfahren hat, als Zurückführung - bei Kant Merkmal philosophischer Methode beschreibbar. Im Gegensatz zu Kant läßt sich diese Form der Zurückführung Beck zufolge nicht als ein Erklären auffassen:

"Da der Geometer, indem er auf keinen Erklärungsgrund achtet, und bloß mit seinem geometrischen Verfahren die Gewißheit verbindet, so muß in diesem Verfahren selbst die Antwort liegen. Dasselbe ist nun kein anderes als das ursprüngliche Vorstellen. [...] Diese Zurückführung einer abgeleiteten Vorstellung auf ein ursprüngliches Vorstellen ist das Wesen einer geometrischen Beweisart." ${ }^{12}$

Beck weist darauf hin, daß die "Gewißheit" im geometrischen Verfahren nicht auf dem Erschließen von Gründen beruht, sondern "die Antwort" liege in dem Verfahren, das mit dem ursprünglichen Vorstellen identifiziert wird. Erinnert sei hier zunächst an die zeitgenössische Interpretation des geometrischen Beweisverfahrens, wie sie Schwab in den "Gedanken über die Analysis" als Einleitung zu Euklids Data in der von ihm 1780 übersetzten Ausgabe von Simson vorliegt. ${ }^{13}$ Danach gehören zu einer geometrischen Aufgabe drei "wesentliche Stücke": Satz, Konstruktion und Beweis. Der Satz bezieht sich auf das Datum, indem er anzeigt, "was gegeben ist und was zu thun gefordert wird". In der

\footnotetext{
Beck (1796) 233.

Beck (1794) 465.

Beck (1796) 204f.

Beck (1796) 203.

Beck (1796) 128.

13 Schwab (1780) §7.
} 
Terminologie des prokleisch interpretierten Beweismodells ${ }^{14}$ entspricht dem Satz die nähere Beschreibung der protasis durch die ekthesis (das Datum) und den dihorismos (Spezifizierung der Aufgabe oder der Behauptung). Durch die Konstruktion (kataskeue) wird die "Forderung" eingelöst. Der Beweis (apodeixis) zeigt, daß die "Forderung durch das Verrichtete wirklich" eingelöst worden ist. Kurzgefaßt läßt sich die interne Struktur des Beweisverfahrens so beschreiben: "Der Satz fragt; die Construction antwortet; der Beweis zeigt, daß die Antwort richtig ist".

Geht man davon aus, daß Beck - er war Mathematiker ${ }^{15}$ - mit dieser Interpretation vertraut war, so läßt sich sein Hinweis, daß "die Antwort" (nämlich auf die Frage, worin die Gewißheit des geometrischen Verfahrens gründe) in dem Verfahren selbst liege, eben auf die "wesentlichen Stücke" beziehen, die für die Antwort, d.h. Einlösung der Forderung, bedeutsam sind: Konstruktion und Beweis. Daß nach Schwabs Darlegung beide Schritte tatsächlich eng aufeinander $\mathrm{zu}$ beziehen sind, geht aus einer Erläuterung hervor: "Construction und Beweis werden mit einem gemeinschaftlichen Namen Composition genannt." Wenn Beck nun das geometrische Verfahren mit dem ursprünglichen Vorstellen gleichsetzt, das Konstruieren als ursprüngliches Vorstellen interpretiert und die Zurückführung von Vorstellungen auf das ursprüngliche Vorstellen als das Wesen der geometrischen Beweisart bezeichnet, dann bezieht sich der Begriff des ursprünglichen Vorstellens nicht auf den eigentlich Konstruktion genannten Schritt im euklidischen Beweismodell, sondern auf die Composition von Konstruktion und Beweis (kataskeue und apodeixis). Diese Lesart scheint auch Schelling vor Augen zu stehen, wenn er behauptet: "Der Demonstration geht die Construktion nicht voran, sondern beides ist eins und unzertrennlich."16 Ja Kant selbst bezieht sich in A734 B762 offensichtlich auf den besonderen Konnex von Konstruktion und Beweis: "Selbst das Verfahren der Algeber mit ihren Gleichungen, aus denen sie durch Reduktion die Wahrheit zusamt dem Beweise hervorbringt, ist [...] Konstruktion".

Sofern nun Kants Theorem der Konstruktion der Begriffe mit Beck als ein ursprüngliches Vorstellen aufzufassen ist, avanciert der Konstruktionsbegriff selbst zum Typus demonstrativer Erkenntnisart. Schelling scheint wiederum an Becks Interpretation der geometrischen Beweisart anzuschließen, wenn er schreibt:

"Die Geometrie und überhaupt die Mathematik ist bis jetzt die einzige Wissenschaft, welche ein allgemeines Beispiel jener absoluten Erkenntnißart, die wir auch die demonstrative nennen können, gegeben hat. Die geometrische Evidenz beruht auf der völligen Aufhebung des Causalgesetzes, sie erklärt nicht, z.B. wie es komme, daß in einem Dreieck dem größeren Winkel immer die größere Seite gegenüber liege, daß je zwei Seiten eines Dreiecks zusammengenommen größer seyen als die dritte, sie beweist, daß es so sey." ${ }^{17}$

Nach dieser Erklärung läßt sich freilich der spekulative Gehalt und der Vollzug der absoluten Erkenntnisart, die Schelling eben die philosophische Konstruktion nennt, nicht

14 Vgl. 4.2.

15 Nach Erdmann (1931) 537 habilitierte sich Beck 1791 "durch Vertheidigung des Taylor'schen Lehrsatzes" in Halle.

16 System 1802: SW IV 407. - Nach Lorenz (1971) 882 ist das Wort Beweis 1670 anstelle von demonstratio (apodeixis) in die mathematische Fachsprache eingeführt worden.

17 System 1802: SW IV 345. 
bestimmen. Wie in 6.2. dargelegt, entspricht dies auch nicht der Absicht dieser Untersuchungen. Hingegen kann diese Konzeption der Terminologie wie auch der systematischen Funktion nach präzise bestimmt werden. Daß Schelling die absolute als demonstrative Erkenntnisart, Konstruktion zugleich als Demonstration versteht, macht deutlich, daß die Transformation des Konstruktionsbegriffs bereits vollzogen ist. So will Kant noch streng zwischen Beweis und Demonstration unterschieden wissen:

\begin{abstract}
"Allein aus Gründen a priori kann sie, als Philosophie, zwar beweisen, aber nicht demonstrieren; wenn man nicht ganz und gar von der Wortbedeutung abgehen will, nach welcher demonstrieren (ostendere, exhibere) so viel heißt, als (es sei in Beweisen oder auch bloß im Definieren) seinen Begriff zugleich in der Anschauung darstellen: welche, wenn sie Anschauung a priori ist, das Konstruieren desselben heißt, wenn sie aber auch empirisch ist, gleichwohl die Vorzeigung des Objekts bleibt, durch welche dem Begriffe die objektive Realität gesichert wird." ${ }^{18}$
\end{abstract}

Unter der Ausformulierung des Konstruktionstheorems als Darstellung des Begriffs in der Anschauung a priori versteht Kant eine Form der Demonstration. Kant erläutert die Demonstration in der Perspektive der transzendentalen Theorie der Erfahrung. Danach zielt die Demonstration eines Begriffs auf die Sicherung seiner objektiven Realität, d.h. den Nachweis, daß der Begriff etwas bezeichnet, dessen Existenz in Raum und Zeit bestimmt werden kann. Läßt sich der Begriff in der Anschauung a priori darstellen, spricht Kant geradezu von Demonstration als Konstruktion des Begriffs.

Erfolgt die Darstellung des Begriffs "auch empirisch", d.h. auch in der Anschauung a posteriori, spricht Kant zwar nicht mehr von Konstruktion, doch gleichwohl von der Demonstration des Begriffs zum Nachweis der objektiven Realität des Begriffs. Somit ist auch die Philosophie der Demonstration fähig, wennauch nicht "allein aus Gründen a priori"; die Anschauung, in der der Begriff dargestellt wird, muß dann "auch empirisch" sein. Schelling hält die Unterscheidung von philosophischer und mathematischer Beweismethode nach der Apriorität und Aposteriorität der Darstellung von Begriffen für unzulässig, da diese Differenz innerhalb der philosophischen Theorie, die sie formuliert, nicht begründbar ist. Denn Apriorität, Empirizität, Objektivität sind Begriffe, deren Bedeutung immer nur auf ein ihnen Entgegengesetztes verstanden werden kann. Die Analysen in Kap. 8 im Ausgang von Schellings Kritik am Begriff des Empirischen haben gezeigt, daß für unser durch Begriffe vermitteltes Verstehen von Sachverhalten zwar die Entgegensetzung der Momente in Verhältnisse von Bedingung und Bedingtem, Bestimmung und Bestimmtem konstitutiv ist, aber eben deswegen im Hinblick auf eine Letztbegründung aporetisch verlaufen muß. Daß unser Verstehen dennoch Gewißheit beanspruchen kann, versucht Schelling durch den Nachweis einer der Entgegensetzung zugrundeliegenden absoluten Vereinigung oder ursprünglichen Identität der entgegengesetzten Momente zu belegen. Als Methode, diese Identität aufzuweisen, dient die absolute Konstruktion: "Das, wodurch Construktion absolut ist, ist mit dem, was Princip des Zusammenhangs der philosophischen Demonstration ist, selbst identisch und ein und dasselbe." 19

$18 K U \S 57$ Anm.I: AA V $241=$ WW V 448f.; vgl. A734f. B762.

19 System 1802: SW IV 409. 
Die Transformation des Konstruktionsbegriffs wird in seiner Interpretation als Demonstration deutlich. Diese Interpretation eröffnete Schelling eine Perspektive, von der "Selbstkonstruktion" des Prinzips der Philosophie, ja sogar vom "Selbstbeweis" der Philosophie zu sprechen. ${ }^{20}$ Mit der Transformation sollte zwar das Ziel, die Begründung des Prinzips der Kantischen Transzendentalphilosophie $\mathrm{zu}$ geben, erreicht sein. Wie Schelling die Transformation vollzogen hat, konnte an seiner Auseinandersetzung mit Becks Kant-Interpretation gezeigt werden. Daß Schelling die Transformation gerade so vollzieht, daß Konstruktion zum Prinzip philosophischer Demonstration wird, ist, so meine These, durch Jacobis Vernunftkritik, die eine Kritik der philosophischen Demonstration ist, veranlaßt worden. Im Kontext dieser Kritik hat Jacobi in der "Beylage VII" der zweiten Auflage seiner Schrift Ueber die Lehre des Spinoza in Briefen an den Herrn Moses Mendelssohn (1789) seine wissenschaftskritische Interpretation des Konstruktionstheorems vorgetragen. ${ }^{21}$ Dieser Interpretation gilt es sich zunächst zuzuwenden, um dann abschließend Schellings These der Selbstkonstruktion des Prinzips der Transzendentalphilosophie als einer demonstrativen Erkenntnisart zu explizieren.

\subsection{Jacobis Kritik der demonstrativen Methode}

Neben Beck war Jacobis Interpretation des Kantischen Konstruktionstheorems geeignet, Schelling die Richtung einer Transformation des Konstruktionsbegriffs zu weisen. Beck und Jacobi heben die Funktion hervor, die Kants Theorem für unsere rationale Weltdeutung der Geometrisierung der Natur hat. Ist für Jacobi ein Begreifen naturwissenschaftlicher Sachverhalte ohne Rekurs auf das Verfahren mathematischer Konstruktion nicht möglich, so legt Beck diese hermeneutische Funktion noch tiefer, indem er Konstruktion als ein ursprüngliches Vorstellen deutet, das unseren Begriffen 'eigentlich Sinn und Bedeutung gibt'. Beck liefert Schelling gewissermaßen das Interpretament zum Verständnis der in den $M A N W$ vorgeführten 'Konstruktion der Materie'. Es ist jedoch Jacobi, der vor dem Hintergrund der von Beck geleisteten Verortung des Konstruktionsproblems in den $M A N W$ die Perspektive vorgibt, den Konstruktionsbegriff nicht nur aus dem mathematischen Kontext herauszulösen, sondern auch als philosophischen Methodenbegriff operabel zu machen.

Nun entwickelt Schelling seine Lesart der Konstruktion als Demonstration nicht in der Auseinandersetzung mit Jacobi, sondern mit Kant, und zwar in der Absicht, Kants Unternehmen der Transzendentalphilosophie eine Deutung zu geben, die sie gegen die Einwände Jacobis absichern sollte. In der "Beylage VII" der Spinoza-Briefe (1789) will Jacobi das, was wir "begreifen" nennen, als ein auf Demonstration beruhendes Verfahren entlarven, das ein Erkennen wirklicher Sachverhalte nur vorspiegelt und gerade

20 System 1802: SW IV 371.

21 Zur Bedeutung, die Jacobi über die Rolle des Spinoza-Vermittlers hinaus für die Entstehung des Idealismus als dessen "negativem Wegbereiter" zukommt, s. Sandkaulen-Bock (1990) 13-18. Henrich (1991) 205-213 weist darauf hin, daß die "Beylage VII" der Spinoza-Briefe für die Diskussion der Grundlagen der Kantischen Philosophie im Tübinger Stift zentral ist. 
ausschließt. Doch aus dem, was Jacobi gerade als Einwand gegen jede Form demonstrativer Methode verstanden wissen wollte, gewinnt Schelling die Instrumente, mit denen er das Unternehmen einer Transzendentalphilosophie gegen diesen Einwand verteidigen zu können glaubt. Dies impliziert freilich, daß Schelling, nicht anders als Jacobi, Kants transzendentale Theorie als eine Form demonstrativer Methode auffaßt, die es durch weiterführende Interpretationen, etwa im Zuge der Transformation des Konstruktionsbegriffs, erst zu erschließen und so gegen Kants eigene Grenzbestimmungen durchzusetzen gilt. Nur deshalb kann Schelling der Meinung sein, "daß Kant, wenn er ein vollendetes Bewußtseyn seiner Philosophie hätte und der Reflexion darüber fähig wäre, construiren müßte". ${ }^{22}$

In seiner Analyse "der speculativen Vernunft"23 und "des menschlichen Erkenntnißvermögens"24 stößt Jacobi auf ein dem Menschen eigenes "Bedürfniß der Abstraction und Sprache"25. So schwinge sich der Mensch zum Schöpfer "einer Vernunftwelt" auf,

"worin Zeichen und Worte die Stelle der Substanzen und Kräfte vertreten. Wir eignen uns das Universum zu, indem wir es zerreissen, und eine unseren Fähigkeiten angemessene, der wirklichen ganz unähnliche Bilder-Ideen- und Wort-Welt erschaffen. [...] was sich auf diese Weise nicht erschaffen läßt, verstehen wir nicht". ${ }^{26}$

Solches Verstehen kennzeichnet Jacobi als "Unterschiede setzen und wieder aufheben"27, das so auf die bekannten Reflexionsgegensätze von Form und Materie, Subjekt und Objekt, Bedingung und Bedingtes führe. ${ }^{28}$ Das Begreifen beruhe auf dem Prinzip, "da $\beta$ alles Bedingte eine Bedingung haben müsse". ${ }^{29}$ Nun führt aber die Anwendung dieser Denkfigur in die Aporien entweder des unendlichen Regresses oder dessen Abbruch durch Setzung eines unbegreiflichen Unbedingten. Wollen wir daher Bedingtes "begreiflich, das heißt natürlich erklären"30, so verfangen wir uns zwangsläufig "in einer Kette bedingter Bedingungen"31 und in einer "Unendlichkeit von Vermittlungen"32, die uns "nie einen wirklichen Anfang, nie ein reelles Prinzip"33 erschließen lassen.

Die apodiktische Gewißheit sowie die allgemeine und notwendige Geltung dieses Prinzips führt Jacobi darauf zurück, daß nach diesem Prinzip Erschlossenes bloß Identisches aussage. Was wir so zu wissen vorgeben, sei das, was die Vernunft "in identischen Sätzen darstellt". ${ }^{34}$ Jacobi glaubt, es sei nur die "Unbestimmtheit Menschlicher Sprache und Bezeichnung", die uns vorspiegele, als sagten diese Sätze "etwas mehr, als das bloße:

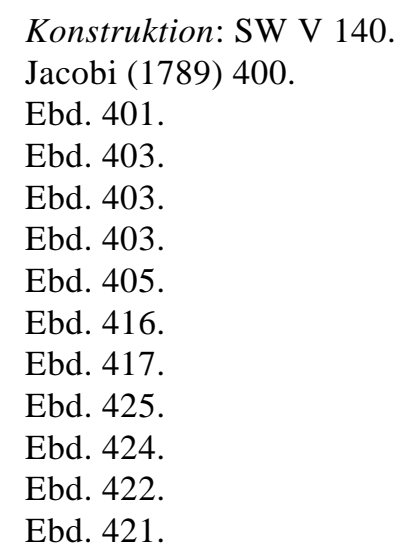


quidquid est, illud est; mehr, als ein bloßes Factum aus, welches wahrgenommen, beobachtet, verglichen, wieder erkannt, und mit anderen Begriffen verknüpft wurde." ${ }^{35}$ Der Versuch, uns den Begriff der Möglichkeit des Daseins der Natur, den absoluten Anfang oder Ursprung der Natur verständlich zu machen, hieße, die "Bedingungen des Unbedingten entdecken [...] und es construiren zu wollen, um es begreifen zu können" ${ }^{136}$ :

"Die Construction eines Begriffes überhaupt ist das a priori aller Constructionen; und die Einsicht in seine Construction giebt uns zugleich auf das gewisseste zu erkennen, daß wir unmöglich begreifen können, was wir zu construiren nicht im Stande sind. Darum haben wir von Qualitäten, als solchen, keine Begriffe; sondern nur Anschauungen. Selbst von unserem eigenen Daseyn haben wir nur ein Gefühl; aber keinen Begriff. Eigentliche Begriffe haben wir nur von Figur, Zahl, Lage und Bewegung, und den Formen des Denkens. Wenn wir sagen, daß wir eine Qualität erforscht haben, so sagen wir damit nichts anders, als wir haben sie auf Figur, Zahl, Lage und Bewegung zurückgeführt, und darin aufgelöst; also: wir haben die Qualität objectiv vernichtet." ${ }^{37}$

Konstruktion der Begriffe deutet Jacobi als den Versuch, dasjenige, wovon wir "keine Begriffe; sondern nur Anschauungen" haben, d.h. Qualitäten, auf das 'zurückzuführen', wovon wir "eigentliche Begriffe" haben: Figur, Zahl, Lage, Bewegung und die Formen des Denkens. Jacobis Analyse des Kantischen Konstruktionstheorems besagt demnach, "daß wir unmöglich begreifen können, was wir zu construiren nicht im Stande sind." - für Jacobi ein ganz und gar untauglicher Versuch, Welt zu verstehen und Naturforschung zu treiben. ${ }^{38}$

Wenn wir uns z.B. eine unendliche Zahlenfolge durch "mathematische Gleichnisse" verständlich machen wollten, so sei eben diese Sukzession "keine objective und wirkliche, sondern eine subjective und blos idealische, die auch nicht einmal idealisch vorhanden seyn könnte, wenn ihr nicht eine wirkliche Succeßion in dem Subject, welches sie in Gedanken erzeugt, zum Grunde läge, und dadurch das Stehende in ein Fließendes verwandelt würde." ${ }^{39}$ Für Jacobi steht fest, daß das, was jeweils als denknotwendig Unbedingtes vorausgesetzt sei, "auf keine andre Weise von uns angenommen werden [kann], als es uns gegeben ist; nehmlich, als Thatsache - Es ist!"40 Damit ist denn auch Jacobis Glaubenssatz seiner "Unphilosophie" ausgesprochen, den er bewußt gegen die cartesianische Tradition der Bewußtseinsphilosophie formuliert. Es scheint, als habe er geahnt, daß er auf diese Weise geradezu zum "negativen Wegbereiter des Idealismus" werden sollte. ${ }^{41}$

Er bekennt, "daß ich kein Cartesianer bin. Ich gehe, wie die Morgenländer in ihren Conjugationen von der dritten, nicht von der ersten Person aus, und glaube, man dürfe schlechterdings nicht das sum dem cogito nachsetzen. Vielleicht sind wir der Periode nahe, wo es möglich seyn wird, eine sehr lehrreiche Geschichte des berühmten Cartesianischen Axioms zu entwerfen. Noch ist es schwer, diesen Proteus zu fassen; und vielleicht war es nie gefährlicher, ihn binden zu wollen." 42

35 Ebd. 421.

36 Ebd. 424f.

37 Ebd. 420*.

38 Vgl. Moiso (1985).

39 Jacobi (1789) 407f.

40 Ebd. 427.

41 Sandkaulen-Bock (1990) 13-18.

42 Jacobi (1789) XXIVf. - Diese visionäre Passage findet sich nur in der Originalausgabe der 2. Aufl. der Spinoza-Briefe. In Bd. IV,1 der von Roth/Köppen herausgegebenen Werke F.H. Jacobis, Leipzig 1819 (Nachdruck Darmstadt 1980) fehlt die gesamte "Vorrede" zur 2. Aufl. der Spinoza-Briefe; vgl. Henrich (1991) 205-213. 
Es ist nun zu zeigen, wie Schelling diese Kritik Jacobis am Verfahren philosophischer Demonstration in seiner Neuinterpretation des Konstruktionstheorems so aufnimmt, daß Konstruktion, verstanden als Prinzip der Demonstration, dem Anspruch nach zu einer letztbegründenden Instanz der Transzendentalphilosophie Kants werden kann.

\subsection{Selbstkonstruktion}

In der Höijer-Rezension vertritt Schelling die These,

"daß alle Demonstration nichts anders als Herbeyführung des Puncts, wo das Identische und Synthetische Eins ist, oder allgemeine Zurückführung der Synthesis auf die reine Identität des Denkens überhaupt ist." ${ }^{43}$

Zur Erläuterung dieser These verweist Schelling auf eine Stelle im System des transzendentalen Idealismus (1800). Die Passage, auf die Schelling sich bezieht, ist unschwer auszumachen, nimmt sie doch, zudem durch Sperrdruck hervorgehoben, die Formulierung der Rezension vorweg. Schelling spricht hier von einem "Widerspruch", der nur dadurch aufzulösen wäre,

"daß irgend ein Punkt gefunden würde, worin das Identische und Synthetische Eins ist, oder irgend ein Satz, der, indem er identisch, zugleich synthetisch, und, indem er synthetisch, zugleich identisch ist." 44

Diese Passage leitet den fünften Schritt der "Deduktion des Princips selbst" ein, das das "Princip des transscendentalen Idealismus" ist. Der Widerspruch, den es durch Auffinden eines Punktes, "worin das Identische und Synthetische Eins ist", aufzulösen gilt, besteht in der Unvereinbarkeit der Forderungen, die ein Prinzip, soll es das höchste der Transzendentalphilosophie - verstanden als "Wissenschaft alles Wissens" ${ }^{45}$ - sein, erfüllen muß. Als höchstes Prinzip des Wissens soll es "unbedingt" und doch Gegenstand des Wissens sein können, dessen Prinzip es ist. Der Transzendentalphilosoph, so führt Schelling aus, suche "das Princip des Wissens innerhalb des Wissens (es ist also selbst etwas, das gewußt werden kann)." ${ }^{46}$ Wie Schelling den besagten Widerspruch herleitet, soll in zwei Schritten entwickelt werden:

(a) Wenn Wissen, so die Voraussetzung, "auf der Uebereinstimmung eines Objektiven und Subjectiven" beruhe und wenn diese Übereinstimmung näher als "ein Zusammentreffen von Entgegengesetzten" (Subjekt und Objekt) bestimmt wird, so könne dieses, wie es heißt, "wirkliche", "ursprüngliche" oder "eigentliche" Wissen ${ }^{47}$ nicht unmittelbar, sondern nur durch "etwas allgemein Vermittelndes"48 als wahr und real betrachtet werden. Diese

43 Konstruktion: SW V 138.

44 System 1800: SW III 363.

45 System 1800: SW III 354.

46 System 1800: SW III 355.

47 System 1800: SW III 353, 362.

48 System 1800: SW III 353. 
Übereinstimmung Entgegengesetzter werde in synthetischen Sätzen der Form A=B ausgedrückt. ${ }^{49}$

(b) Nun sind synthetische Sätze gerade deswegen, weil sie der Vermittlung bedürfen, nicht unbedingt. Unbedingt oder "durch sich selbst gewiß" sind "identische oder analytische Sätze" der Form A=A..$^{50}$

Soll das Prinzip des Wissens selber Gegenstand des Wissens sein, so müßte es, als Gewußtes, selber etwas "unbedingt Gewisses" darstellen, d.h. nach den angeführten Voraussetzungen identisch und synthetisch zugleich sein. Schellings Auflösung dieses Widerspruchs besteht in dem Postulat des Begriffs eines "Subjekt-Objekts" in Form des Satzes "Ich=Ich", der eine Gleichheit zwischen dem Ich als Subjekt und dem Ich als Objekt ausdrückt. Der Ausdruck "Ich=Ich" verwandele den identischen Satz A=A in einen synthetischen, da, durch den Begriff des Ich substituiert, "das eine A dem andern A entgegengesetzt" werde. Es wird sich als Subjekt und Objekt selbst entgegengesetzt. Aus der Substitution ergibt sich für den identischen Satz der Form A=A eine synthetische Interpretation, so daß das Ich aufgrund der ihm zugeschriebenen Selbstbezüglichkeit in dem Fall, da es sich selbst Objekt wird, zugleich "nichtobjektiv" ist. ${ }^{51}$ "Das UnbedingtGewisse kann also für sie [die Wissenschaft des Wissens] nur in dem absolut Nichtobjektiven liegen, welches auch die Nichtobjektivität der identischen Sätze (als der einzig unbedingt gewissen) beweist. Wie nun aber aus diesem ursprünglich Nichtobjektiven ein Objektives entspringe, wäre nicht zu begreifen, wenn nicht jenes Nichtobjektive ein Ich wäre, d.h. ein Princip, das sich selbst Objekt wird." ${ }^{52}$

Im folgenden interessiere ich mich nicht für die zugegeben problematischen Annahmen dieser Prinzipienspekulation. Sie läuft, wenig überraschend, darauf hinaus, das Prinzip des Seins und des Erkennens zusammenfallen zu lassen ${ }^{53}$, und bietet als methodisches Instrument des Nachvollzugs, ebensowenig überraschend, die intellektuelle Anschauung an. ${ }^{54}$ Was für Schelling den Vorteil dieser Theorie ausmacht, nämlich daß mit dem Begriff des Ich als Prinzip der Garant der Vergewisserung zugleich das Kriterium der Überprüfung sein soll ${ }^{55}$, entpuppt sich in den Augen des Rezipienten dieser Theorie als entscheidender Nachteil: Als Theorie der absoluten Wahrheit verfügt sie über keine Theorie des Irrtums, Irrtum vermag sie nur durch Verweis auf das Fehlen und den Mangel des Organs der intellektuellen Anschauung zu erklären. ${ }^{56}$ Dadurch ist es ihr weder möglich, die behaupteten Sachverhalte im Diskurs zu vermitteln, noch können sie einer angemessenen Kritik im Diskurs unterzogen werden. Von Interesse für meine Fragestellung ist allerdings die "Beschreibung" des gesuchten Punktes, von dem Schelling behauptet, mit dem Begriff

49 System 1800: SW III 363.

50 System 1800: SW III 362.

51 System 1800: SW III 372.

52 System 1800: SW III 374.

53 System 1800: SW III 368.

54 System 1800: SW III 369.

55 System 1800: SW III 369: "Eine solche [intellektuelle] Anschauung ist das Ich, weil durch das Wissen des Ichs von sich selbst das Ich selbst (das Objekt) erst entsteht." - SW III 370: "Das Ich selbst ist ein Objekt, das dadurch ist, daß es von sich weiß, d.h. es ist ein beständiges intellektuelles Anschauen".

56 System 1800: SW III 369f. 
des Ich als Prinzip der Philosophie sei zugleich die Selbstkonstruktion des Prinzips bezeichnet. ${ }^{57}$ Hatte Schelling schon in der Übersicht behauptet, "daß das Ich ursprünglich die Construction von sich selbst ist" ${ }^{\prime 5}$, so bezeichnet er das Prinzip der Transzendentalphilosophie in der Form des Satzes "Ich=Ich" als eine dem Ich "eigne Construktion". ${ }^{59} \mathrm{Um} \mathrm{zu}$ erläutern, wie Schelling zu dieser Auszeichnung des Prinzips kommt, gehe ich in drei Schritten vor: (a) Beschreibung des Prinzips: Das höchste Prinzip der Transzendentalphilosophie ist von einem der Transzendentalphilosophie immanenten Standpunkt aus beschreibbar. (b) Deduktion des Prinzips, die Schelling als den Weg "vom Wissen zum Seyn" im "Uebergang vom Subjekt zum Objekt" mit Hilfe eines "Experimentes" ausweist. ${ }^{60}$ (c) Schelling kommt durch die urteilslogische Interpretation des "Ich bin" als eines "unendlichen Satzes" zur Rede von der Selbstkonstruktion des Ich. Das "Ich bin" wird als "ursprüngliche Position" zum Datum und Ansatzpunkt der Konstruktion gemacht.

(a) Beschreibung des Prinzips: Prinzip der Transzendentalphilosophie ist Schelling zufolge das Selbstbewußtsein, das, verstanden als "Wissen von uns", zugleich das "erste Wissen für uns" sei. ${ }^{61}$ Mit der Formulierung dieses Sachverhaltes deutet sich bereits an, worauf Schelling mit seiner Ausgangsfrage nach dem Prinzip, das zugleich Objekt des Wissens, dessen Prinzip es ist, sein soll, hinaus will: Aus der formalen Bestimmung des Prinzips der Philosophie als systematischer Wissenschaft soll die materiale Bestimmung des Prinzips der Philosophie als Wissenschaft des Wissens abgeleitet werden können. Dabei wird Selbstbewußtsein als "Art des Wissens", nicht als "Art des Seyns" expliziert. Daraus resultiert eine "Beschränkung" des methodischen Zugangs für die Beschreibung des Prinzips, der von einem Verfahren der Abstraktion begleitet wird:

\footnotetext{
"Daß nun aber dieses Selbstbewußtseyn nur die Modification eines höheren Seyns [...] vielleicht eines höheren Bewußtseyns [...] seyn könne - mit Einem Wort, daß auch das Selbstbewußtseyn noch etwas überhaupt Erklärbares seyn möge, erklärbar aus etwas, von dem wir nichts wissen können, [...] geht uns als Transscendental-Philosophen nichts an" ${ }^{12}$.
}

Schelling schließt solche höheren Formen des Seins und Bewußtseins als Erklärungsgrund des Selbstbewußtseins nicht aus, markiert aber entschieden Grenze und Zugangsart des Standpunktes, von dem aus die Theorie des transzendental genannten Idealismus zu entwickeln ist. Damit versucht er offensichtlich der Kant-Kritik Jacobis zu entsprechen, ohne Kants idealistische Grundoption aufzugeben. Denn er trägt Jacobis Einwänden gegen die Aporien eines diskursiven Verfahrens, das als Begreifen von Bedingungen durch Vermittlung von Begriffen konstituiert ist, Rechnung. ${ }^{63}$ Zugleich hält er sich jedoch mit der offensichtlich zulässigen Rede von der "Art des Seyns" noch die Option Jacobis offen, so etwas wie ein ursprüngliches Sein als Begründungsinstanz einzuführen. Andererseits

57 System 1800: SW III $371 \mathrm{f}$.

58 Übersicht: SW I 448 = AW IV 175.

59 System 1800: SW III 371.

60 System 1800: SW III 358f.

61 System 1800: SW III 355.

62 System 1800: SW III 355f.

63 System 1800: SW III 369: "Dieses Wissen muß [...] ein absolut-freies seyn [...], wozu nicht Beweise, Schlüsse, überhaupt Vermittlung von Begriffen führen". 
gewinnt er durch die Neuinterpretation der transzendentalen Apperzeption eine Erweiterung der Grenzen innerhalb der Grenzbestimmung der Vernunft. Schelling knüpft dabei an Kants Überlegungen an, daß mit der Rede von der Grenze auf etwas Positives auch in dem, was ich nicht wissen kann, verwiesen ist, während die Schranke die Negation dessen ist, was ich nicht weiß. ${ }^{64}$ Nach der Kantischen Sprachregelung setzt die Rede von der Grenze die Vorstellung eines Raumes voraus, der außerhalb eines bestimmten Platzes liegt und denselben einschließt. Nun verwendet Schelling die Begriffe "Begrenzung" und "Beschränkung" anders als Kant unterschiedslos, hebt aber der Sache nach auf die Funktion des Grenzbegriffs als eines Forschungsprinzips ab. Schelling kennzeichnet das Prinzip als epistemologisches, nicht als ontologisches, was aus der "Behauptung: es gibt ein höchstes Princip des Wissens" nun "eine negative, einschränkende Behauptung, in der nur so viel liegt: es gibt irgend ein Letztes, [...] jenseits dessen kein Wissen ist" macht. Für den Transzendentalphilosophen gilt daher: "ob es, abstrahirt von uns, jenseits dieses ersten Wissen noch überhaupt etwas gebe, kümmert ihn vorerst gar nicht". ${ }^{65}$

Es ist diese "Beschränktheit der Aufgabe"66, die Schellings Unternehmen des transzendentalen Idealismus als das dem Kantischen verpflichtet zeigt. Metaphysik als Wissenschaft zu etablieren, bedeutete für Kant die Restringierung apriorischen Wissens auf die Formulierung der Bedingungen, die eine naturwissenschaftliche Beschreibung unserer Erfahrungen zuläßt. Aus dem Vorrat der traditionellen Metaphysik konnten so Grenzbegriffe als Forschungsprinzipien - Kant spricht von Ideen als "heuristischen Fiktionen" (A771 B799) - gewonnen werden, und zwar im Verbund von Metaphysik, Naturwissenschaft und Mathematik. Die hierbei in Ansatz gebrachten fundamentalen Prinzipien unserer Erkenntnis waren jedoch innerhalb der Kantischen Konzeption selbst nicht Gegenstand der Erkenntnisleistung. Sie wurden zwar als Bedingungen objektiver Erkenntnis erschlossen, entzogen sich aber deswegen der erkennenden Bezugnahme nach eben denselben Prinzipien.

Wenn jedoch das Prinzip des Wissens selbst nicht gewußt werden kann, dann könne es auch nicht die Begründung von Wissen leisten. Schellings methodologische Einschränkung besteht in der Abstraktion "von allem Objektiven", denn der Transzendentalphilosoph mache "überall nur das Subjektive sich zum Objekt". ${ }^{67}$ Schellings Konzept geht davon aus, daß das Prinzip des Wissens innerhalb des Wissens aufzusuchen sei: "Der Transscendental-Philosoph fragt nicht: welcher letzte Grund unseres Wissens mag außer demselben liegen? sondern: was ist das Letzte in unserem Wissen selbst, über das wir nicht hinauskönnen? - Er sucht das Princip des Wissens innerhalb des Wissens (es ist also selbst etwas, das gewußt werden kann)." ${ }^{68}$ Den Weg zu einer solchen Deduktion schlägt er mit Hilfe eines "Experimentes" ein. ${ }^{69}$

$64 \mathrm{Vgl} .1 .3$.

65 System 1800: SW III 355.

66 System 1800: SW III 354, 357.

67 System 1800: SW III 354f.

68 System 1800: SW III 355.

69 System 1800: SW III 359. 
(b) Deduktion des Prinzips: Mit Hilfe eines Experimentes will Schelling das Prinzip der Philosophie als ein solches ausweisen, in dem Form und Inhalt sich wechselseitig bedingen.

"Man denke sich irgend einen formellen Satz, z.B. A=A, als den höchsten; was an diesem Satze logisch ist, ist bloß die Form der Identität zwischen A und A; aber woher kommt mir denn A selbst? Wenn A ist, so ist es gleich sich selbst; aber woher ist es denn? Diese Frage kann ohne Zweifel nicht aus dem Satz selbst, sondern nur aus einem höheren beantwortet werden. Die Analysis A=A setzt die Synthesis A voraus. ${ }^{170}$

Nach Schellings Urteilstheorie werden in jedem Satz "zwei Begriffe miteinander verglichen, d.h. sie werden einander entweder gleich oder ungleich gesetzt. Im identischen Satze nun wird bloß das Denken mit sich selbst verglichen."71 Dieser Vergleich erfolgt jedoch, wie Schelling in Anspielung auf Jacobi hervorhebt, "ohne alle Vermittlung". ${ }^{72}$ Schelling fügt hinzu: "was denn ohne Zweifel durch einen Denkakt geschieht. Der Satz $\mathrm{A}=\mathrm{A}$ setzt also ein Denken voraus, das unmittelbar sich selbst zum Objekt wird". Es gehe hierbei darum, daß man "durch Reflexion auf den Denkakt in diesem Satze etwas Reelles [...] und so aus jedem identischen Satz den Akt des Selbstbewußtseyns finden könne."73

Versteht man Schelling so, daß logische Verhältnisse tatsächlich auf ein psychologisch zu beschreibendes Ereignis von 'Akten' zwar nicht reduziert, doch auch nicht unabhängig davon expliziert werden können, so zieht Schelling den Verdacht des Psychologismus auf sich. Schelling spricht denn auch vom "Übergang des Wissens zum Sein", den dieses Experiment offenbare. ${ }^{74}$ Stellt man diesen Einwand zurück und betrachtet das hier beschriebene Modell als ein Modell, das die Struktur von Selbstbewußtsein, verstanden als "Art des Wissens", in der Form eines Urteils ausdrücken soll, läßt sich deutlich machen, inwiefern Schelling zugleich von der Selbstkonstruktion des Selbstbewußtseins als Prinzip sprechen kann.

(c) Interpretation des Prinzips: Schelling unterscheidet zwei "Arten von Bewußtseyn": Er grenzt das reine Bewußtsein oder Selbstbewußtsein von dem bloß empirischen Bewußtsein $\mathrm{ab}$, "was wir insgemein Bewußtseyn nennen". ${ }^{75}$ Das empirische Bewußtsein sei

"etwas nur an Vorstellungen von Objekten Fortlaufendes, was die Identität im Wechsel der Vorstellungen unterhält, also bloß empirischer Art, indem ich dadurch freilich meiner selbst, aber nur als des Vorstellenden bewußt bin." ${ }^{76}$

Es ist deutlich, daß Schelling hier an Bestimmungen der Schrift Vom Ich anknüpft. Dort hatte er das, was Kant synthetische Einheit des Selbstbewußtseins nennt, deswegen als empirisch-bedingt bezeichnet, weil es in dem Akt des 'Ich denke' stets einen intentionalen Gegenstand habe und korrekt als etwas, das "Objecte denkt", interpretiert werden muß.77 Die analytische Einheit des Selbstbewußtseins besagt, daß das Ich, das Bewußtsein von

\footnotetext{
70 System 1800: SW III 360.

System 1800: SW III 364.

System 1800: SW III 364.

System 1800: SW III 365.

System 1800: SW III 368.

75 System 1800: SW III 366f.

76 System 1800: SW III 366.

77 Vom Ich §8: SW I 180* = AW II 105*. Vgl. 8.2.
} 
sich hat, sich im Hinblick auf jede der wechselnden verschiedenen Vorstellungen, die es hat, als dasselbe begreift. Kant formuliert als Bedingung für diesen Sachverhalt: "Das: Ich denke, muß alle meine Vorstellungen begleiten können". (B131)

Denn sofern das Ich in dem Satz 'Ich denke' sich nur als eines Denkenden oder etwas Vorstellenden bewußt ist, muß gelten, daß es einen zweifachen Bezug zu den Vorstellungen, deren es sich bewußt ist, hat. Erstens durch die Beziehung des 'Ich denke' zu jeder einzelnen Vorstellung aus dem Gesamt der Vorstellungen, deren es sich bewußt ist. Zweitens durch die Einheit, die der erstgenannten insofern vorausgeht, als es die Verknüpfung unter den einzelnen Vorstellungen, die durch das 'Ich denke' begleitet werden können, als Gesamt zu denken möglich macht. Diese zweite Bedingung von Selbstbewußtsein nennt Kant die ursprünglich synthetische Einheit des Selbstbewußtseins oder transzendentale Synthesis der Apperzeption, weil sie der analytischen Einheit vorgängig ist. Schelling deutet diese Einheit des Ich als empirisches Bewußtsein des Ich, genauer des empirisch-bedingten Ich, sofern es nämlich "Objecte denkt" und nur in Beziehung auf Objekte zu explizieren ist. Davon unterscheidet er das reine Bewußtsein oder Selbstbewußtsein überhaupt, das er als den "Akt" verstanden wissen will, "wodurch ich meiner nicht mit dieser oder jener Bestimmung, sondern ursprünglich bewußt werde". ${ }^{78}$ Zwischen beiden Arten des Bewußtseins sieht er eine "Genese", die er wiederum durch ein Experiment verdeutlicht:

\footnotetext{
"Man überlasse sich ganz der unwillkürlichen Succession der Vorstellungen, so werden diese Vorstellungen, so mannichfaltig und verschieden sie seyn mögen, doch als zu Einem identischen Subjekt gehörig erscheinen. Reflektire ich auf diese Identität des Subjekts in den Vorstellungen, so entsteht mir der Satz: Ich denke. Dieses Ich denke ist es, was alle Vorstellungen begleitet und die Continuität des Bewußtseyns zwischen ihnen unterhält. - Macht man aber von allem Vorstellen sich frei, um seiner ursprünglich bewußt zu werden, so entsteht - nicht der Satz: Ich denke, sondern der Satz: Ich bin, welcher ohne Zweifel ein höherer Satz ist." ${ }^{79}$
}

Der Satz 'Ich bin' soll jener Akt sein, in dem das Ich sich seiner "ursprünglich bewußt" wird. Daß Schelling mit dem 'Ich bin' auf die Existenz des Ich hinweist, unterscheidet ihn freilich noch nicht von Kant. Auch Kant spricht von einem "actus purus", in dem sich das "daß ich bin" des Ich manifestiere. ${ }^{80}$ Deutlicher formuliert Kant diesen in $K r V \S \S 17$ und 25 angedeuteten Gedanken in B422f.:

\footnotetext{
"Das Ich denke, ist [...] ein empirischer Satz, und enthält den Satz, Ich existiere, in sich. [...] die Existenz ist hier noch keine Kategorie, als welche nicht auf ein unbestimmt gegebenes Objekt, sondern nur ein solches, davon man einen Begriff hat, und wovon man wissen will, ob es auch außer diesem Begriffe gesetzt sei, oder nicht, Beziehung hat. Eine unbestimmte Wahrnehmung bedeutet hier nur etwas Reales, das gegeben worden [...] als etwas, was in der Tat existiert, und in dem Satze, ich denke, als ein solches bezeichnet wird."
}

78 System 1800: SW III 367.

79 System 1800: SW III 367.

80 "[... bin ich mir meiner selbst in der transzendentalen Synthesis des Mannigfaltigen der Vorstellungen überhaupt, mithin in der synthetischen ursprünglichen Einheit der Apperzeption, bewußt, nicht wie ich mir erscheine, noch wie ich an mir selbst bin, sondern nur daß ich bin. Diese Vorstellung ist ein Denken, nicht ein Anschauen." (B157) 
Es ist die Interpretation des Satzes, die Schelling von Kant unterscheidet. ${ }^{81}$ Für Kant war das 'Ich denke', weil es sich nicht erscheint, nicht intelligibel, die Selbstanwendung und begründung als Prinzip der Philosophie ist damit Schelling zufolge ausgeschlossen. Für Schelling ist das 'Ich denke' wegen seiner Objektbeziehung empirisch-bedingt. Diese (mögliche) Beziehung war für Kant jedoch gerade die Bedingung dafür, Objektivität zu begründen:

"Denn es ist zu merken, daß, wenn ich den Satz: ich denke, einen empirischen Satz genannt habe, ich
dadurch nicht sagen will, das Ich in diesem Satz sei empirische Vorstellung, vielmehr ist sie rein
intellektuell, weil sie zum Denken überhaupt gehört. Allein ohne irgendeine empirische Vorstellung,
die den Stoff zum Denken abgibt, würde der Aktus, Ich denke, doch nicht stattfinden, und das
Empirische ist nur die Bedingung der Anwendung, oder des Gebrauchs des reinen intellektuellen
Vermögens." (B423*)

Nun bietet Schelling für den Satz 'Ich bin' eine urteilslogische Interpretation an, die ihm zugleich erlaubt, das durch diesen Satz beschriebene Prinzip als Selbstkonstruktion zu verstehen:

"In dem Satz: Ich denke, liegt schon der Ausdruck einer Bestimmung oder Affektion des Ich; der Satz: Ich bin, dagegen ist ein unendlicher Satz, weil es ein Satz ist, der kein wirkliches Prädicat hat, der aber eben deßwegen die Position einer Unendlichkeit möglicher Prädicate ist." ${ }^{12}$

Schelling schreibt dem Kantischen 'Existentialsatz' urteilslogische Qualitäten zu: Er charakterisiert ihn als einen "unendlichen Satz", der zwar kein wirkliches Prädikat habe, aber die Position einer Unendlichkeit möglicher Prädikate sei. Neben den bejahenden und verneinenden Urteilen, die aus der formalen Logik bekannt sind, führt Kant in der transzendentalen Logik eine dritte Art von Urteilen auf, die unendlichen Urteile, die sonst zu den bejahenden gezählt werden. Die formale Logik abstrahiere vom Inhalt der Begriffe, die an der Prädikatstelle im Urteil auftreten, und sehe nur darauf, ob das Prädikat "dem Subjekt beigelegt, oder ihm entgegengesetzt werde." (A72 B97) Man sagt dann, daß im bejahenden Urteil "das Subjekt unter der Sphäre eines Prädikats gedacht", im verneinenden Urteil werde es "außer der Sphäre des letzteren gesetzt". ${ }^{3}$ Die transzendentale Logik jedoch "betrachtet das Urteil auch nach dem Werte oder Inhalt dieser logischen Bejahung vermittelst eines bloß verneinenden Prädikats, und was diese in Ansehung des gesamten Erkenntnisses für einen Gewinn verschafft." (A72 B97) Diesen Typ unendlicher Urteile verdeutlicht Kant, allerdings in Abgrenzung zur Art der verneinenden Urteile, folgendermaßen: "Alles Mögliche ist entweder A oder non A. Sage ich also: etwas ist non A, z.B. die menschliche
Seele ist nicht-sterblich, [...] so ist dies ein unendliches Urteil. Denn es wird durch dasselbe über die
endliche Sphäre A hinaus nicht bestimmt, unter welchen Begriff das Objekt gehöre; sondern
lediglich, daß es in die Sphäre außer A gehöre, welches eigentlich gar keine Sphäre ist, sondern nur
die Angrenzung einer Sphäre an das Unendliche oder die Begrenzung selbst. - Obgleich nun die
Ausschließung eine Negation ist: so ist doch die Beschränkung eines Begriffs eine positive
Handlung. Daher sind Grenzen positive Begriffe beschränkter Gegenstände." 84

81 Schelling kommentiert diese Textstelle bereits in der Übersicht: SW I 401f. = AW IV 128. Darauf weist schon Frank (1985) 36-47 mit der Vermutung hin, daß Schelling wohl der einzige unter den Idealisten sei, "dem jene merkwürdige Anmerkung [...] nicht entgangen ist." (42)

82 System 1800: SW III 367.

$83 \quad L$ \$22: AA IX $103=$ WW III 534.

${ }^{84} L$ §22: AA IX 104 = WW III 534f.; vgl. A71f. B97f. 
Bei der urteilslogischen Bestimmung des Prinzips der Philosophie mit dem Satz 'Ich bin' schließt Schelling an die Merkmale, mit denen Kant die Qualität unendlicher Urteile beschreibt, gleich mehrfach an: Schelling kennzeichnet das Prädikat, mit dem er das Prinzip beschreibt, näher als "an sich nichtobjektiv" und das, was es bezeichnet, als "das ins Unendliche fort Nichtobjektive". ${ }^{85}$ Darin, dieses Prinzip als das höchste des Wissens zu bestimmen, liegt nach Schelling ja gerade die "Beschränktheit der Aufgabe" des Transzendentalphilosophen; denn er begreift die Behauptung, daß es ein höchstes Prinzip des Wissens gebe, genau als "eine negative, einschränkende Behauptung, in der nur so viel liegt: es gibt irgend ein Letztes, [...] jenseits dessen kein Wissen ist." ${ }^{86}$ Mit der Rede von dem 'Ich bin' als "Position" kann sich Schelling wiederum die Konvergenz der mathematikund urteilstheoretischen Terminologie zunutze machen. Die Position im geometrischen Sinne bezeichnet ja gerade das Datum, mit dem die Konstruktion beginnt. ${ }^{87}$ Das 'Ich bin' bezeichnet so den unbedingt gegebenen Ausgangspunkt der Transzendentalphilosophie, der, in die Form eines identischen Urteils A=A gebracht, durch Substitution des A durch den Begriff Ich aufgrund der ihm zugeschriebenen Selbstbezüglichkeit synthetisch interpretiert werden kann. Sofern es sich hierbei tatsächlich um die Formation eines Urteils handelt und Formation, wie in Kap.7 dargelegt, gerade Konstruktion bedeutet, kann Schelling so auch von der Selbstkonstruktion im Sinne der ursprünglichen Konstruktion des Prinzips sprechen. ${ }^{88}$

Da nun das Ich "kein anderes Prädicat hat als das des Selbstbewußtseyns"89, wird es nur sich selbst zum Objekt, dessen Begriff etwas repräsentiert, "das zugleich sich selbst entgegensetzt, und sich selbst gleich ist." ${ }^{10}$ Es drückt damit eine Beziehung aus, die Schelling, anknüpfend an seine Bestimmung des Prinzips des transzendentalen Idealismus in der Übersicht, als 'ursprüngliche Duplizität in der Identität' umschreibt und in der Schrift Vom Ich schon mit dem Theorem der 'Gleichsetzung von Ich und Nicht-Ich' als Ausdruck theoretischer Erkenntnis belegt.

Zusammenfassung: 'Konstruktion der Begriffe' lautet der Titel eines philosophischen Programms, das zu erklären versucht, warum die Natur geometrisch geordnet werden kann. Kants transzendental- und Schellings identitätsphilosophische Theorien einer uns erfahrbaren Natur sind der Versuch, Entstehung und Fortschritt der mathematischen Naturwissenschaft und damit die Geometrisierung der Natur als integrierenden Bestandteil unserer rationalen Weltdeutung $\mathrm{zu}$ begreifen. Es ist zugleich der Versuch, sich das philosophischer Deduktion überlegene Konzept mathematischer Konstruktion durch die Art seiner philosophischen Interpretation verfügbar $\mathrm{zu}$ machen. Durch die rezeptionsanalytischen Untersuchungen konnten Argumentationsverläufe bis in einzelne sprachliche Wendungen hinein nachvollziehbar gemacht werden. Es lassen sich zwei

85 System 1800: SW III 367f.; vgl. 8.2.

86 System 1800: SW III 354f., 357.

87 Vgl. 9.1.

88 System 1800: SW III 371.

89 System 1800: SW III 368.

$90 \quad$ System 1800: SW III 373. 
Ergebnisse herausstellen: Schellings Redeweise ist durch den Gebrauch einer Reihe fachsprachlicher Begriffe geprägt, deren mathematiktheoretische Implikationen noch näher zu erkunden wären. Aus der Rezeptionsanalyse ließ sich jedoch die heuristische Perspektive für die vorgelegte Kant-Interpretation gewinnen. Die These, daß Kant "construire, ohne es zu wissen" (Höijer) oder "construiren müßte" (Schelling), deutet auf eine Fehlinterpretation der Kantischen Differenz von mathematischer und philosophischer Erkenntnisart hin. Danach hätte Kant die Differenz beider Erkenntnisarten absolut gesetzt und sich die Möglichkeit genommen, das Kriterium der Differenz in der Theorie, die die Differenz formuliert, auch zu begründen. Diese fruchtbare Fehlinterpretation mußte aus Sicht der Rezipienten, nicht aus der Sicht Kants, eine Neuinterpretation des Konstruktionsbegriffs in den $M A N W$ nahelegen. 


\section{Siglen und Kurztitel}

Werkausgaben, die durch Siglen bezeichnet werden:

AA Kant's Werke, hg. Preußische Akademie der Wissenschaften, Berlin 1902- .

AW F.W.J. Schelling: Historisch-kritische Ausgabe der Bayerischen Akademie der Wissenschaften, Reihe I Werke, 4 Bde. [ersch.], Stuttgart 1976- .

SS B.C.H. Höijers samlade Skrifter, [hg. J. Höijer], 5 Bde., Stockholm 1825-1827.

SW F.W.J. v. Schellings sämmtliche Werke, hg. K.F.A. Schelling, 14 Bde., Stuttgart/Augsburg 1856-61.

WW Immanuel Kant: Werke, hg. W. Weischedel, 6 Bde., Darmstadt 1983. [Diese Studienausgabe ist text- und seitengleich mit der Theorie-Werkausgabe, 12 Bde., Frankfurt/M. 1968 und der Paperback-Ausgabe, 10 Bde., Darmstadt 1983.]

BuD F.W.J. Schelling. Briefe und Dokumente, hg. H. Fuhrmans, 2 Bde., Bonn 1962/1973.

Werke Kants, die durch Siglen bezeichnet werden:

$\boldsymbol{E} \quad$ Über eine Entdeckung, nach der alle neue Kritik der reinen Vernunft durch eine ältere entbehrlich gemacht werden soll (1790) [Streitschrift gegen Eberhard]

$\boldsymbol{F}$

Welches sind die wirklichen Fortschritte, die die Metaphysik seit Leibnizens und Wolffs Zeiten in Deutschland gemacht hat? (1791/1804) [Preisschrift]

$\boldsymbol{K} \boldsymbol{r} \boldsymbol{K} \quad$ Kritik der reinen Vernunft $\left({ }^{1} 1781=\mathrm{A} /{ }^{2} 1787=\mathrm{B}\right)$

Kritik der Urteilskraft (1790)

L $\quad$ Logik (1800)

MANW Metaphysische Anfangsgründe der Naturwissenschaft (1786)

MARL Metaphysische Anfangsgründe der Rechtslehre (1797) [= Metaphysik der Sitten. Erster Teil]

$\boldsymbol{O P}$ Opus postumum [Kants handschriftlicher Nachlaß 1796-1803]

$\boldsymbol{P}$

Prolegomena zu einer jeden künftigen Metaphysik, die als Wissenschaft wird auftreten können (1783) 
Werke Schellings, die durch Kurztitel bezeichnet werden:

Ideen Ideen zu einer Philosophie der Natur (1797/1803)

Konstruktion Über die Konstruktion in der Philosophie (1803) [Rez. von B.C.H. Höyer[!]: Abhandlung über die philosophische Construction, Stockholm 1801.]

System $1800 \quad$ System des transzendentalen Idealismus

System $1801 \quad$ Darstellung meines Systems der Philosophie

System 1802 Fernere Darstellungen aus dem System der Philosophie

Übersicht Allgemeine Übersicht der neuesten philosophischen Literatur (1797/1798) [= Abhandlungen zur Erläuterung des Idealismus der Wissenschaftslehre]

Vom Ich Vom Ich als Prinzip der Philosophie (1795)

\section{Zitierweise und Zeichen}

Werkausgaben werden nach Sigle, Band (römische Ziffer) und Seitenzahl (arabische Ziffer) zitiert. Kant wird in der Regel nach WW zitiert mit Angabe der Band- und Seitenzahl von WW und AA in den Fußnoten. Ausnahme: Zitate aus der $K r V$ werden im Text nachgewiesen mit Angabe der Auflage und Seitenzahl nach I. Kant: Kritik der reinen Vernunft, hg. R. Schmidt, Hamburg 1976.

Schelling wird, soweit erschienen, nach AW zitiert mit Angabe der Band- und Seitenzahl von AW und SW in den Fußnoten.

Die Forschungsliteratur wird mit Verfasser, Jahr (in Klammern) und Seitenzahl zitiert.
kursiv Hervorhebung im zitierten Text
[ ] Hinzufügung J.W.
[...] Auslassung J.W.
[* ] in den zitierten Text eingeschobene Fußnote desselben Textes 


\section{Literaturverzeichnis}

Adickes, E. (1924): Kant als Naturforscher, 2 Bde., Bd. I, Berlin.

Arndt, H.W. (1971): Methodo scientifica pertractatum. Mos geometricus und Kalkülbegriff in der philosophischen Theorienbildung des 17. und 18. Jh.s, Berlin/New York.

Barnaby, D.C. (1988): "The Early Reception of Kant's Metaphysical Foundations of Natural Science", in: Metaphysics and Philosophy of Science in the Seventeenth and Eighteenth Centuries, ed. R.S. Woolhouse, Dordrecht usw.

Beck, J.S. (1793, 1794, 1796): Erläuternder Auszug aus den critischen Schriften des Herrn Prof. Kant, auf Anrathen desselben, 3 Bde., Riga. [Titel Bd. III: Einzig-möglicher Standpunkt, aus welchem die critische Philosophie beurtheilt werden muß]

Beck, J.S. (1795): "Fragmente aus einer noch ungedruckten Schrift, welche den Titel führt: Einzig möglicher Standpunkt, aus welchem die kritische Philosophie beurtheilt werden muß", in: Philosophischer Anzeiger, 24-31 (1795) 185-246 [Beilage zu Annalen der Philosophie und des philosophischen Geistes von einer Gesellschaft gelehrter Männer, hg. L.H. Jakob]

Beth, E.W. (1956/57): "Über Lockes 'Allgemeines Dreieck'", in: Kant-Studien, 48 (1956/57) 361-380.

Böhme, G. (1986): Philosophieren mit Kant. Zur Rekonstruktion der Kantischen Erkenntnis- und Wissenschaftstheorie, Frankfurt/M.

Boenke, M. (1990): Transformation des Realitätsbegriffs. Untersuchungen zur frühen Philosophie Schellings im Ausgang von Kant, Stuttgart-Bad Cannstatt.

Brittan, G.G. (1978): Kant's Theory of Science, Princeton.

Buchdahl, G. (1984): "Zum Verhältnis von allgemeiner Metaphysik der Natur und besonderer metaphysischer Naturwissenschaft bei Kant", in: Probleme der "Kritik der reinen Vernunft", hg. B. Tuschling, Berlin/New York, 97-142.

Buchner, H. (1965): "Hegel und das Kritische Journal der Philosophie", in: Hegel-Studien, 3 (1965) 95156.

Büchel, G. (1987): Geometrie und Philosophie, Berlin/New York.

Butts, R.E. (1986) (ed.): Kant's Philosophy of Physical Science. Metaphysische Anfangsgründe der Naturwissenschaft 1786-1986, Dordrecht usw.

Capozzi Cellucci, M. (1973): "J. Hintikka e il methodo della mate-matica in Kant", in: Il Pensiero, 18 (1973) 232-267.

Carl, W. (1989): Der schweigende Kant. Die Entwürfe zu einer Deduktion der Kategorien vor 1781, Göttingen.

Carrier, M. (1990): "Kants Theorie der Materie und ihre Wirkung auf die zeitgenössische Chemie", in: Kant-Studien, 81 (1990) 170-210.

Cramer, K. (1985): Nicht-reine synthetische Urteile a priori, Heidelberg.

Cramer, K. (1986): "'Gegeben' und 'Gemacht'. Vorüberlegungen zur Funktion des Begriffs 'Handlung' in Kants Theorie der Erkenntnis von Objekten", in: Handlungstheorie und Transzendentalphilosophie, hg. G. Prauss, Frankfurt/M. 
Cramer, K. (1987): "Über den Satz: Das: Ich denke, muß alle meine Vortsellungen begleiten können", in: Theorie der Subjektivität, hg. K. Cramer u.a., Frankfurt/M., 167-202.

Ende, H. (1973): Der Konstruktionsbegriff im Umkreis des Deutschen Idealismus, Meisenheim/Glan.

Engfer, H.-J. (1982): Philosophie als Analysis. Studien zur Entwicklung philosophischer Analysiskonzeptionen unter dem Einfluß mathematischer Methodenmodelle im 17. und frühen 18. Jh., Stuttgart-Bad Cannstatt.

Erdmann, B. (1878): Kants Kriticismus in der 1. und 2. Auflage der Kritik der reinen Vernunft, Leipzig (Nachdruck Hildesheim 1973).

Erdmann, B. (1937): Versuch einer wissenschaftlichen Darstellung der Geschichte der neuern Philosophie, 3. Abt. Bd. I, Stuttgart (Leipzig $\left.{ }^{1} 1848\right)$.

Falkenburg, B. (1987): Die Form der Materie. Zur Metaphysik der Natur bei Kant und Hegel, Frankfurt/M.

Fichte, J.G. (1794/95): Grundlage der gesammten Wissenschaftslehre, Studientextausgabe [Teilausgabe der Fichte-Gesamtausgabe der Bayerischen Akademie der Wissenschaften, Bd. I.2] Stuttgart-Bad Cannstatt 1969.

Fichte, J.G. (1794-1812): Nachgelassene Schriften zu Platners "Philosophischen Aphorismen" 1794-1812, Fichte-Gesamtausgabe, Bd. II.4, Stuttgart-Bad Cannstatt 1976.

Frank, M. (1985): Eine Einführung in Schellings Philosophie, Frankfurt/M.

Frank, M./Kurz, G. (1975) (Hg.): Materialien zu Schellings philosophischen Anfängen, Frankfurt/M.

Frede, M./Krüger, L. (1970): "Über die Zuordnung der Quantitäten des Urteils und der Kategorien der Größe bei Kant", in: Kant-Studien, 61 (1970) 28-49.

Friedman, M. (1986): "The Metaphysical Foundations of Newtonian Science", in: Kant's Philosophy of Physical Science: metaphysische Anfangsgründe der Naturwissenschaft, hg. R. E. Butts, Dordrecht u.a.

Gierow, K.R. (1971): Benjamin Höijer, Stockholm.

Gierow, K.R. (1971-1973): "Höijer, Benjamin Carl Henrik", in: Svensk Biografiskt Lexikon, Bd. IXX, Stockholm, 693-696.

Gloy, K. (1976): Die Kantische Theorie der Naturwissenschaft, Berlin/New York.

Gloy, K. (1990): "Der Substanzsatz in der 'Kritik der reinen Vernunft' und in den 'Metaphysischen Anfangsgründen der Naturwissenschaft'", in: Gloy, K.: Studien zur theoretischen Philosophie Kants, Würzburg, 82-114.

Heath, Th.L. (1926): The Thirteen Books of Euclid's Elements, trans., intro., commentary, 2 vols., 2nd ed., rev. with additions, Cambridge $\left({ }^{1} 1908\right)$.

Heckmann, R./Krings, H./Meyer, R.W. (1985) (Hg.): Natur und Subjektivität. Zur Auseinandersetzung mit der Naturphilosophie des jungen Schelling, Stuttgart-Bad Cannstatt.

Hegel, G.F.W. (1802): Kritisches Journal der Philosophie, Bd. I.3, in: Gesammelte Werke, Bd. IV, Hamburg 1968.

Hegel, G.F.W. (1807): Phänomenologie des Geistes, in: Gesammelte Werke, Bd. IX, Hamburg 1980.

Heiberg (1969) (Hg.): Euklid. Elementa, ed. E.S. Stamatis, post I.L. Heiberg, vol. I, Bibliotheca Scriptorum Graecorum et Romanorum Teubneriana, 2. Aufl., Leipzig.

Heller, E. (1993): "Kant und J.S. Beck über Anschauung und Begriff", in: Philosophisches Jahrbuch, 100 (1993) 72-95.

Henrich, D. (1976): Identität und Objektivität. Eine Untersuchung über Kants transzendentale Deduktion, Heidelberg. 
Henrich, D. (1991): Konstellationen. Probleme und Debatten am Ursprung der idealistischen Philosophie (1789-1795), Stuttgart.

Hintikka, J. (1969): "Kant on the Mathematical Method", in: Kant Studies Today, ed. L.W. Beck, La Salle/Ill., 117-140.

Hintikka, J. (1984): "Kant's Transcendental Method and His Theory of Mathematics", in: Topoi, 3 (1984) 99-107.

[Höijer] Hoyer, B.C.H. (1801): Abhandlung über die philosophische Construction, als Einleitung zu Vorlesungen in der Philosophie, aus dem Schwedischen, Stockholm.

Höijer, B.C.H. (1898): Ur Benjamin Höijers Manuskript. Excerpt, hg. E. Leufvén, Gefle.

Hoppe, H. (1969): Kants Theorie der Physik, Frankfurt/M.

Horstmann, R.-P. (1991): Die Grenzen der Vernunft. Eine Untersuchung zu Zielen und Motiven des Deutschen Idealismus, Frankfurt/M.

Jacobi, F.H. (1789): Über die Lehre des Spinoza in Briefen an den Herrn Moses Mendelssohn, 2., neue verm. Ausg., Breslau $\left({ }^{1} 1785\right)$.

Jacobs, W.G. (1988): "Einleitung" zu F.W.J. Schelling. Einleitung zu seinem Entwurf eines Systems der Naturphilosophie, Stuttgart, 3-12.

Jammer, M. (1981): Der Begriff der Masse in der Physik, übers. H. Hartmann, 3. Aufl., Darmstadt $\left({ }^{1} 1964\right)$. [Concepts of Mass, Cambridge/Mass. 1961]

Jergius, H. (1975): Philosophische Sprache und analytische Sprachkritik. Bemerkungen zu Fichtes Wissenschaftslehren, Freiburg/München.

Koethen, J.J. (1737): Principia quaedam metaphysicae Wolfianae variis observationibus illustrata. Accedunt, propter argumenti similitudinem, Theses Metaphysiscae Leibnitianae [...], Genève.

Krausser, P. (1981): Kants Theorie der Erfahrung und Erfahrungswissenschaft, Frankfurt/M.

Krings, H. (1982): "Die Konstruktion in der Philosophie. Ein Beitrag zu Schellings Logik der Natur", in: Aspekte der Kultursoziologie, hg. J. Stagl, Berlin, 341-351.

Krings, H. (1985): "Natur als Subjekt. Ein Grundzug der spekulativen Philosophie Schellings", in: Heckmann/Krings/Meyer (1985) 111-128.

Leibniz, G.W. (1695): Specimen Dynamicum, hg. und übers. H.G. Dosch u.a., erl. J. Aichelin u.a., Hamburg 1982.

Leibniz, G.W. (1715/16): "Streitschriften zwischen Leibniz und Clarke", in: Die philosophischen Schriften, hg. C.I. Gerhardt, Bd. VII, Berlin 1890 (Nachdruck Hildesheim 1978).

Leute, R. (1972): Grundwissen Physik A/B, Stuttgart.

Loer, B. (1974): Das Absolute und die Wirklichkeit in Schellings Philosophie, Berlin/New York.

Löw, R. (1979): "Qualitätenlehre und Materiekonstruktion. Zur systematischen Aktualität von Schellings Naturphilosophie", in: Schelling. Seine Bedeutung für eine Philosophie der Natur und der Geschichte, hg. L. Hasler, Stuttgart-Bad Cannstatt, 99-106.

Lorenz, K. (1971): "Beweis", in: Historisches Wörterbuch der Philosophie, Bd. 1, hg. J. Ritter, Darmstadt, Sp. 882-886.

Lukács, G. (1955): Die Zerstörung der Vernunft. Der Weg des Irrationalismus von Schelling zu Hitler, Berlin.

Lütterfelds, W. (1975): "Deduktion und Konstruktion. Kants widersprüchliche Theorie des geometrischen Apriori", in: Kant-Studien, 66 (1975) 418-445. 
Mainzer, K. (1970): "Der Konstruktionsbegriff in der Mathematik", in: Philosophia Naturalis, 12 (1970) 367-412.

Martin, G. (1972): Arithmetik und Kombinatorik bei Kant, Berlin/New York.

Mendelssohn, M. (1785): Morgenstunden oder Vorlesungen über das Daseyn Gottes, in: Gesammelte Schriften, Jubiläumsausgabe, hg. L. Strauss, Bd. III.2, Stuttgart 1974.

Moiso, F. (1985): "Schellings Elektrizitätslehre 1797-1799", in: Heckmann/Krings/Meyer (1985) 59-97.

Mudroch, V. (1978): Kants Theorie der physikalischen Gesetze, Berlin/New York.Newton, I. (1872): Mathematische Prinzipien der Naturlehre, übers. J.Ph. Wolfers, Berlin (Nachdruck Darmstadt 1963). [Philosophiae naturalis principia mathematica, 3. Aufl., 1726]

Niebel, E. (1959): Untersuchungen über die Bedeutung der geometrischen Konstruktion in der Antike, Köln. [Kant-Studien Erg.H., 76]

Nordin, S. (1987): Romantikens filosofi. Svensk idealism från Höijer till hegeliarnerna, Lund.

Ordbok över svenska språket, utg. Svenska Akademien, Bd. XVIII, Lund 1926, Art. "formera", 1148-1151.

Parsons, Ch. (1969): "Kant's Philosophy of Arithmetic", in: Philosophy, Science, and Method, ed. S. Morgenbesser/P. Suppes/M. White, New York, 568-594.

Parsons, Ch. (1984): "Arithmetic and the Categories", in: Topoi, 3 (1984) 109-121.

Peters, W.S. (1962): "Zum Begriff der Konstruierbarkeit bei I. Kant", in: Archive for History of Exact Science, 2 (1962) 153-167.

Plaaß, P. (1965): Kants Theorie der Naturwissenschaft, Göttingen.

Pötschel, W. (1910): Jakob Sigismund Beck und Kant, Diss. Breslau.

Reinhold, K.L. (1789): Versuch einer neuen Theorie des menschlichen Vorstellungsvermögens, Prag/Jena.

Reinhold, K.L. (1791): Ueber das Fundament des philosophischen Wissens, Jena.

Reinhold, K.L. (1797): Auswahl vermischter Schriften, Teil 2, Jena.

Reinhold, K.L. (1803): Beyträge zur leichtern Uebersicht des Zustandes der Philosophie beym Anfange des 19. Jahrhunderts, Hamburg, 208-213. [Rez. von Höijer (1801)]

Salvucci, P. (1958): Grandi interpreti di Kant. Fichte e Schelling, Urbino.

Sandkaulen-Bock, B. (1990): Ausgang vom Unbedingten. Über den Anfang in der Philosophie Schellings, Göttingen.

Schäfer, L. (1966): Kants Metaphysik der Natur, Berlin.

Schirn, M. (1991): "Kants Theorie der geometrischen Erkenntnis und die nichteuklidische Geometrie", in: Kant-Studien, 82 (1991) 1-28.

Schmucker-Hartmann, J. (1976): Der Widerspruch von Vorstellung und Gegenstand. Zum Kantverständnis von Jakob Sigismund Beck, Meisenheim/Glan.

Schneiders, W. (1983): Aufklärung und Vorurteilskritik. Studien zur Geschichte der Vorurteilstheorie, Stuttgart-Bad Cannstatt.

Schulthess, P. (1981): Relation und Funktion, Berlin/New York.

Schwab, J.Chr. (1780): "Gedanken über die Analysis" [\$§ 1-30, ohne Seitenzählung], in: Euklids Data (verb. und verm. R. Simson), aus dem Engl. übers., und mit einer Samml. geometrischer, nach der Analytischen Methode der Alten aufgelößter Probleme begleitet, Stuttgart.

Steck, M. (1945): Proclus Diadochus: Kommentar zum ersten Buch von Euklids "Elementen", Einl. und Komm. [übers. P.L. Schönberger], Halle. 
Stegmaier, W. (1980): "Kants Theorie der Naturwissenschaft", in: Philosophisches Jahrbuch, 87 (1980) 363-377.

Stolzenberg, J. (1986): Fichtes Begriff der intellektuellen Anschauung. Die Entwicklung der Wissenschaftslehren von 1793/94 bis 1801/02, Stuttgart.

Stuhlmann-Laeisz, R. (1973): "Über Kants Problem der 'Anwendung der Kategorien' durch den 'Schematismus des reinen Verstandes'", in: Archiv für Geschichte der Philosophie, 55 (1973) 301-309.

Taureck, B. (1975): Schicksal der philosophischen Konstruktion, Wien/München.

Thaer, C. (1980): Euklid: Die Elemente. Buch I-XIII, übers. und hg., 7. Aufl., Darmstadt.

Tuschling, B. (1971): Metaphysische und transzendentale Dynamik in Kants opus postumum, Berlin/New York.

Verra, V. (1979): "La 'construction' dans la philosophie de Schelling", in: Actualité de Schelling, publ. G. Planty-Bonjour, Paris.

Waidhas, D. (1985): Kants System der Natur, Frankfurt/M. usw.

Weizsäcker, C.F. v. (1966): "Kants Theorie der Naturwissenschaft nach P. Plaaß", in: Kant-Studien, 56 (1966) 528-544.

Wieland, W. (1975): "Die Anfänge der Philosophie Schellings und die Frage nach der Natur", in: Frank/Kurz (1975) 237-279.

Wolters, G. (1980): Basis und Deduktion. Studien zur Entstehung und Bedeutung der Theorie der axiomatischen Methode bei J.H. Lambert (1728-1777), Berlin/New York.

Young, J.M. (1982): "Kant on the Construction of Arithmetical Concepts", in: Kant-Studien, 73 (1982) 1746. 